\title{
A coupled bispectral, temporal and spatial coherence function of the pressure field, scattered from a moving sea surface $(A)$
}

Bjerrum-Niese, Christian; Jensen, Leif Bjørnø

Published in:

Acoustical Society of America. Journal

Link to article, DOI:

$10.1121 / 1.414267$

Publication date:

1995

Document Version

Publisher's PDF, also known as Version of record

Link back to DTU Orbit

Citation (APA):

Bjerrum-Niese, C., \& Jensen, L. B. (1995). A coupled bispectral, temporal and spatial coherence function of the pressure field, scattered from a moving sea surface (A). Acoustical Society of America. Journal, 98(5), 28962897. https://doi.org/10.1121/1.414267

\section{General rights}

Copyright and moral rights for the publications made accessible in the public portal are retained by the authors and/or other copyright owners and it is a condition of accessing publications that users recognise and abide by the legal requirements associated with these rights.

- Users may download and print one copy of any publication from the public portal for the purpose of private study or research.

- You may not further distribute the material or use it for any profit-making activity or commercial gain

- You may freely distribute the URL identifying the publication in the public portal 
The 130th Meeting of the Acoustical Society of America

\title{
Adam's Mark Hotel • St. Louis, Missouri • 27 November-1 December 1995
}

NOTE: All Journal articles and Letters to the Editor are peer reviewed before publication. Program abstracts, however, are not reviewed before publication, since we are prohibited by time and schedule.

\section{Session 1aAO}

\author{
Acoustical Oceanography and Underwater Acoustics: Acoustic Interactions with Internal Waves \\ in Shallow Water I \\ James F. Lynch, Chair \\ Woods Hole Oceanographic Institution, 203 Bigelow Building, Woods Hole, Massachusetts 02543
}

Chair's Introduction-8:25

Invited Papers

8:30

1aAO1. Internal waves and tides in shallow water. Albert J. Plueddemann and James F. Lynch (Woods Hole Oceanogr. Inst., 360 Woods Hole Rd., Woods Hole, MA 02543-1541)

The well-known Garrett and Munk description of the internal wave spectrum synthesizes many observations into a spectral model that reproduces the general features of the mid-latitude, deep-ocean internal wave field. The general applicability of this "kinematic" spectral model is related to the dynamic balance of the internal wave field, but the model itself includes no dynamics and is meant to represent only the average or steady-state spectrum, away from the direct influence of sources, sinks, or boundaries. In shallow water, the kinematic model is typically not appropriate, since some or all of the simplifying assumptions about the wave field may be invalidated. Variable topography, strong tidal currents, fronts, and ice cover (at high latitudes) are the principal reasons for these complications, and result in an internal wave field which varies both spatially and temporally from the steady-state kinematic description. The most straightforward way to address the inadequacy of steady-state models for shallow water acoustics experiments is direct observation of the internal wave field at the site. Profiles sufficient to define the "mean" or background density gradient along with time series of temperature and horizontal velocity are the most common observations.

9:00

1aAO2. Internal solitons in the ocean. John R. Apel (Johns Hopkins Univ., Appl. Phys. Lab., Laurel, MD 20726), Lev A. Ostrovsky (Univ. of Colorado, Boulder, CO 80303), and Yuri A. Stepanyants (Inst. of Appl. Phys., Nizhny Novgorod 603600. Russia)

Internal waves (IW) are among the important factors affecting sound propagation in the ocean. A special role may be played by solitary IWs because of their spatial localization and high magnitudes. Here, nonlinear IWs are discussed (a) from the standpoint of soliton theory and (b) from the viewpoint of experimental measurements. First, basic theoretical models for solitary IWs in the ocean 
are described, and various analytical solutions are treated, commencing with the well-known Korteweg-de Vries equation and its important generalizations including effects of rotation, cylindrical divergence, eddy viscosity, shear flows and instabilities, and turbulence. Experimental evidence for the existence of solitons in the upper ocean is presented both for shallow and deep sea regions. The data include radar and optical images and in situ measurements of waveforms, propagation speeds, and dispersion characteristics. It is suggested that internal solitons in the ocean are ubiquitous and are generated primarily by tidal flows over continental shelf breaks, banks, and similar bottom topographic features. [Work supported by ONR, DOD, ISF.]

9:30

1aAO3. The New Jersey shelf shallow water random media propagation experiment. Marshall H. Orr (Acoust. Div., Naval Res. Lab., Washington, DC 20375-5000) and James F. Lynch (Woods Hole Oceanogr. Inst., Woods Hole, MA 02543)

The range-dependent properties of a variety of pulsed acoustic signals $(200-1000 \mathrm{~Hz})$ propagating in a shallow water waveguide (New Jersey shelf, July-Aug. 1995) randomized by internal waves, bottom heterogeneity and surface roughness have been measured during a 27-day interinstitutional experiment. A 40-km acoustic propagation path located along the NSF Ocean Drilling Program seismic line 1003 was instrumented with moored acoustic sources, three moored acoustic vertical line arrays; eight moored thermistor strings; two moored ADCPs; and two surface gravity wave spectrum buoys. The ocean surface was imaged with ship, P-3 aircraft and ERS-1 radars. The water volume was imaged with high-frequency acoustic systems. Water properties were also measured with shipborne ADCP and CTD sensors. Acoustic sources and receiving arrays were positioned with DGPS and acoustic navigation systems. Timing was maintained with Rubidium clocks. The theoretical/experimental rationale for this experiment will be outlined. The anticipated effects of internal wave- and seafloor-induced randomness on the range-dependent properties of the acoustic signals will be summarized. Preliminary results of the measurements will be presented. [Work supported by ONR.]

10:00-10:15 Break

\section{Contributed Papers}

\section{0:15}

1aA04. Amplitude fluctuation effects in acoustic scattering due to shallow water internal waves. Lei Fu (MIT/WHOI Joint Program, Woods Hole, MA 02543) and James F. Lynch (Woods Hole Oceanogr. Inst., Woods Hole, MA 02543)

Amplitude fluctuation effects in acoustic scattering due to shallow water internal waves are examined using both adiabatic and coupled normal mode theory. The discussion is restricted to linear (nonsoliton) internal waves with a Garrett-Munk spectrum in this work. Coherent and incoherent effects, adiabatic versus coupled scattering, and the range, frequency, and internal wave amplitude sensitivity of the scattering will be discussed. Directions for future work will be discussed.

\section{0:30}

1aA05. Upcoming field experiment examining media effects on synthetic aperture sonar-Experimental considerations and preliminary design. Kevin L. Williams, Terry E. Ewart, Frank S. Henyey, Daniel Rouseff, Stephen A. Reynolds, and James Grochocinski (Appl. Phys. Lab., Univ. of Washington, Seattle, WA 98105)

Ocean variability imposes limitations on performance of synthetic aperture sonar (SAS) systems. An experiment to examine these limitations will be carried out in conjunction with the Coastal Mixing and Optics program. The talk begins with a description of the environment anticipated, based on historical data. Using this environmental information, the effects of refraction, internal waves, and turbulence are shown. Acoustic wavefront curvature caused by internal waves is a primary concern. These results have driven the design of the experimental apparatus. An overview of this apparatus is presented. [Work supported by ONR.]

\section{0:45}

1aAO6. The internal waves effect on the vertical noise directionality in shallow water. T. C. Yang and Dirk Tielbuerger ${ }^{a)}$ (Naval Res. Lab., Washington, DC 20375)

Acoustic environment is known to influence the vertical directionality of the ambient noise. For example, in shallow water with a downward refractive (summer) sound-speed profile, the noise vertical directionality is expected to show a deep ( $>10 \mathrm{~dB}$ ) null in the horizontal direction for wind-generated noise below $1 \mathrm{kHz}$. This "horizontal null" is a conse- quence of the fact that low-order modes are weakly excited by the noise sources which are located near the ocean surface. This phenomenon has been experimentally observed and is not expected to occur in an upward refractive (winter) sound-speed profile environment. It is noted, however, that when internal waves are present, the deep null may disappear or sufficiently weaken due to the "strong" mode coupling effect caused by the scattering of the noise field from the internal waves. In the internal wave fields, the conversion of higher-order modes (generated by the surface noise sources) to low-order modes by the internal waves can fill in the deep ( $>10 \mathrm{~dB}$ ) null which would otherwise be expected (theoretically). Numerical calculations of the vertical noise directionality at $<500 \mathrm{~Hz}$ for typical shallow water environments with rock (low loss) and sediment (lossive) bottoms are presented and the potential effects of internal waves on the ambient noise directionality are investigated. Practical situations are discussed for which the smearing of the "horizontal null" in the noise vertical directionality can be experimentally verified. ${ }^{\text {a) }}$ On leave from Forschungsanstalt der Bundeswehr fur Wasserschall-und Geophysik, Klausdorfer Wef 2-3=24, 24148 Kiel, Germany.

\section{1:00}

1aA07. Separation of turbulence velocity and temperature sound-speed variability with reciprocal transmission. David $M$. Farmer and Daniela Di Iorio (Inst. of Ocean Sciences, $9860 \mathrm{~W}$. Saanich Rd., Sidney, BC V8L 1R7, Canada)

Acoustical scintillation results from the combined contribution of scalar (i.e., temperature and salinity) and vector (velocity) fluctuations. Reciprocal transmission allows us to separate these components. In a turbulent flow, in which the measured scales lie within the vertical subrange, the velocity fluctuations can be used to derive the rate of turbulent kinetic energy dissipation. Reciprocal transmission measurements at $67 \mathrm{kHz}$ were recently acquired as part of the U.S. Office of Naval Research "Moderate to High Frequency" research program. The results are interpreted in terms of the effective refractive index structure parameter and compared both with the independent shear probe measurements of Lueck (personal communication) and the recent theoretical analysis of Ostachev [Waves Random Media 4, 403-428 (1994)]. 


\title{
Session 1aPA
}

\section{Physical Acoustics: General I: Nonlinear Topics and Waves in Media}

\author{
Thomas J. Matula, Cochair \\ Applied Physics Laboratory, University of Washington, 1013 N.E. 40th Street, Seattle, Washington 98105 \\ James P. Chambers, Cochair \\ National Center for Physical Acoustics, University of Mississippi, University, Mississippi 38677
}

\section{Contributed Papers}

\section{8:15}

1aPA1. Time-domain modeling of finite-amplitude sound in relaxing fluids. Robin O. Cleveland, Mark F. Hamilton, and David T. Blackstock (Appl. Res. Labs., Univ. of Texas, Austin, TX 78713-8029, and Dept. of Mech. Eng., Univ. of Texas, Austin, TX 78712-1063)

Two similar time-domain computer algorithms are described, one that solves an augmented Burgers equation and the other an augmented $\mathrm{KZK}$ equation. Both codes include the effects of nonlinearity, absorption, and dispersion (both thermoviscous and relaxational). The Burgers code also accounts for geometrical spreading and inhomogeneity of the medium. The KZK code accounts for diffraction in directive sound beams [Lee and Harnilton, J. Acoust. Soc. Am. 97, 906-917 (1995)]. The novel feature of the codes is that they run exclusively in the time domain, even for the calculation of absorption and dispersion due to multiple relaxation phenomena. For a plane step shock in a monorelaxing fluid, the results from the Burgers code are compared with an analytical prediction of Polyakova et al. [Sov. Phys. Acoust. 8, 78-82 (1962)]. Numerical results are also compared with frequency-domain calculations for a plane harmonic wave in a thermoviscous, monorelaxing fluid. Both comparisons reveal excellent agreement. New results include calculations showing the effect of relaxation on the propagation of a pulsed, diffracting, finite-amplitude sound beam. [Work supported by NASA, the Office of Naval Research, and the IR\&D Program at ARL.]

\section{8:30}

1aPA2. The nonlinear interaction of two plane waves in a viscous medium. Zhong-Yue Jiang and James F. Greenleaf (Biodynam. Res. Unit, Dept. of Physiol. and Biophys., Mayo Clinic and Foundation, Rochester, MN 55905)

Earlier studies [P. J. Westervelt, J. Acoust. Soc. Am. 29, 199-203, 934-935 (1957)] of the mutual nonlinear interaction of two plane waves of sound with each other are extended to include the viscous effect. The viscous effect is considered both from the equations of motion and the equation of state of the medium. An analytical solution to the lowest order scattering process is obtained if the viscous effect of second order and higher can be neglected. In fact, it is shown that the scattered density $\rho_{s}$ of two interacting plane waves having the frequencies $\omega_{1}$ and $\omega_{2}$, respectively, satisfies the following equation: $\square_{v}^{2} \rho_{s}=\square_{v}^{2}\left\{c_{0}^{-2} E_{12}\right.$ $+\left[(2 \cos \theta+B / A) / 2 \omega_{1} \omega_{2}(1-\cos \theta)\right] \nabla^{2} W_{12}+\left[D B\left(c_{0}^{2} \omega_{1} \omega_{2}\right)^{-1} / 4 A\right.$ $\left.\left.\times(1-\cos \theta)^{2}\right] \nabla^{2}\left(\partial W_{12} / \partial t\right)+\left[D B(3+\cos \theta) / 2 A c_{0}^{4}(1-\cos \theta)\right]\left(\partial V_{12} / \partial t\right)\right\}$, where $E_{12}, V_{12}$, and $W_{12}$ are, respectively, the total, potential, and special defined energy densities, $D$ is the sound diffusivity, $B$ and $A$ are nonlinearity parameters, $\theta$ is the intersecting angle, and $\square_{v}^{2}$ is a modified $\mathrm{d}^{2}$ Alembertian operator. [Work supported by CA43920 NIH.]
8:45

1aPA3. Finite amplitude sound beam propagation in dense gases. Edward A. Cox (Dept. of Math. Phys., Univ. College Dublin, Belfield, Dublin 4, Ireland) and Alfred Kluwick (Inst. of Fluid Dynam. and Heat Transfer, Technical Univ., Vienna, Austria)

Current studies indicate that fluids with high specific heats may prove useful in a number of technological applications. These fluids have been shown to exhibit a range of new phenomena-the most prominent example probably being the existence of negative shocks (shocks across which the normal stresses decrease). Of particular theoretical interest is the possibility of propagating waves with mixed nonlinearity where for example positive and negative shocks propagate as stable structures within a single wave pulse. Here existing results for essentially plane waves are extended to include the effects of signal variations in directions transverse to the propagation direction. An extended Khoklov-Zabolotskaya-Kuznetsov (eKZK) is derived which models a propagating finite-amplitude sound beam. The extension to mixed nonlinearity predicts the formation of postitive and negative shocks and the existence of sonic shocks (where the shock speed is equivalent to the convected sound speed upstream or downstream of the shock). Numerical results are presented illustrating the combined effects of nonlinearity and diffraction for both time harmonic and pulsed radiators. An analysis of these results is given in terms of propagating characteristic surfaces.

9:00

1aPA4. Influence of phase between original frequencies on the nonlinear generation of new harmonics. Examples of application from nonlinear acoustics. Claes M. Hedberg (Dept. of Mech. Eng., Univ. of Texas, Austin, TX 78712-1063)

It is assumed that the solution to a problem is given in the form of a complex multiple Fourier series infinite in each of the original frequencies. In this paper a simple theorem shows that the absolute values of the individual frequency coefficients in the series are independent of the phase relation between the original frequencies. But the coefficients division into real and imaginary parts is phase dependent. When the relation between the frequencies and original phase is not an irrational number, there exists in the sum several coefficients giving the same frequency, and hence the total amplitude for a specific frequency depends on the phase. An example of application is shown resulting from nonlinear acoustics where, e.g., the importance of original phase for parametric arrays can be estimated. [Work supported by TFR, Sweden.]

\section{9:15}

1aPA5. Underwater explosion shock wave due to a buried charge. Alan Powell (Dept. of Mech. Eng., Univ. of Houston, TX 77204-4792)

The explosive removal of off-shore oil platform structures has an environmental impact on marine life because of the shock waves from the buried charges. For an in-water detonation the similarity parameter 
$\left(W^{1 / 3} / R\right.$ ) is well known ( $W=$ charge weight, $R=$ radius to observation point), with peak pressure $p_{\max }=$ const. $\times\left(W^{1 / 3} / R\right)^{\alpha}, \alpha=$ attenuation coeff. $\approx 1.13$ (Arons, 1954). Combining the similarity method with ray theory gives. for a point distance $a$ above the bottom due to a charge buried distance $b$ below it, $p_{\max }=$ const. $\times\left(W^{1 / 3} / R\right)^{\varepsilon}\{(a+b) / b\}^{\varepsilon-a}$ with $\varepsilon=$ bottom attenuation coeff. Now $p_{\max }$ is a function only of $\left(W^{1 / 3} / R\right)$ only on horizontal planes $(a+b) / b=$ const.; moreover for given $R$, the pressure increases with height $a$ above the bottom. Full-scale measurements by Connor (1990) confirm these findings, also yielding $\varepsilon \approx 1.99$. The pressure now depends more acutely on charge weight, $p_{\max } \sim W^{2 / 3}$; but more weakly on burial depth than $p_{\max } \sim 1 / b^{2.6}$ as sometimes assumed, e.g., in deep water $a \& R \gg b, p_{\max } \sim 1 / b^{0.86}$. Constant peak pressure contours follow $R=$ const. $\times \cos ^{\left(\varepsilon a^{a}\right) \alpha} \theta, \theta=$ angle to vertical.

\section{9:30}

1aPA6. A powerful acoustical source for seismology. Andrey V. Lebedev and Alexander M. Sutin (Hydroacoustic Div., Inst. Appl. Phys., Russian Acad. Sci., 46 Ulyanov str., Nizhny Novgorod 603600, Russia)

To get information about the "entrails" of the Earth it is necessary to have a powerful source of seismic waves. Standard equipment for this purpose is too heavy and rather unstable. In this work the possibility of powerful electrodynamic hydroacoustic transducer usage for seismology is investigated theoretically. To match the transducer with earth, a tube containing water is used. The hydroacoustical transducer is placed inside the tube. This transducer produces waves that are transformed to seismic waves. The open end of the tube is a monopole-type source of primary compression waves. This source is a resonant one, and, according to calculations, the $Q$ factor is of the order 10 . Working resonance frequency of this system is defined by $h=\lambda_{0} / 2$ condition, where $h$ is the height of the tube, and $\lambda_{0}$ is the wavelength of sound waves in water filling the tube. Such design of the source permits the exclusion of Rayleigh wave generation which is very important for volume tomography of the Earth. Varying the height of the water column can easily tune the frequency of radiation.

\section{9:45}

1aPA7. Acoustic reciprocity-type and energy conservation theorems for flow-structure problems. Oleg A. Godin ${ }^{a)}$ (NOAA/Atlantic Oceanogr. and Meteorolog. Lab., 4301 Rickenbacker Cswy., Miami, FL 33149)

Recently the reciprocity principle was proved for acoustic fields in motionless fluid/solid structures [A. N. Norris and D. A. Rebinsky, J. Acoust. Soc. Am. 94, 1714-1715 (1993)] and a flow reversal theorem (FRT) was established for sound and acoustic-gravity waves in 3-D inhomogeneous, moving fluids [O. A. Godin, J. Acoust. Soc. Am. 97, 3396(A) (1995)]. The FRT is counterpart of the reciprocity principle when ambient flow is present. The theorem states symmetry of some field quantity with respect to interchange of the source and receiver positions and the simultaneous reversal of flow. An FRT will be presented which generalizes results of the above-mentioned works to include waves in flow/solid structures of arbitrary geometry. Parameters of the medium are allowed to be spatially inhomogeneous but time independent. Wave propagation and ambient flow are considered as adiabatic thermodynamic processes. It is assumed the prestresses in the solids due to ambient flow are small. Conservation of a quasienergy for the wave is established and its relation to known acoustic energy corollaries as well as to the FRT is discussed. Some possible applications of the general theorems will be considered. [Work supported by NRC.] ${ }^{\text {a) }}$ On leave from P. P. Shirshov Oceanography Institute, Moscow, Russia.

\section{0:00-10:15 Break}

\section{0:15}

1aPA8. Computation of transient Green's functions through plane interfaces. Carsten Draeger and Didier Cassereau (Lab. Ondes et Acoustique, Université Paris 7, E.S.P.C.I., 10 rue Vauquelin, 75231 Paris Cedex 05, France)

This work studies the acoustical field generated by a point source after reflection or transmission by a plane interface. There are two classical approaches to calculate such transient Green's functions, using either a Laplace or a Fourier transform over time. However, the second approach involves numerical integrations that are difficult to carry out because of singularities of the integrand. It is shown that it is possible to avoid this difficulty using an excitation in the form of a temporal Lorentz function of variable width, introducing several judicious variable changes and finally deforming the integration path in the complex plane. By developing the expression of the transmission/reflection coefficient into Taylor's series around suitable points, it is even possible to carry out a piecewise integration analytically, therefore resulting in an approximate expression of the acoustical field as a function of space and time. Since this expression turns out to be in a closed form, it can be evaluated fast and even be manipulated later on.

\section{0:30}

1aPA9. Interaction of shear wave polarization and composite laminate layup: Experiment and modeling. Brent $A$. Fischer and David K. Hsu (Ctr. for Nondestruc. Eval., lowa State Univ., Ames, IA 50011)

The interaction of shear wave polarization and the fiber directions in a composite laminate makes shear waves a sensitive probe for evaluating ply orientation and sequence in a layup. The transmitted shear wave signal, with the transmitting and receiving transducers perpendicular to each other, can easily distinguish a $[0 /+45 / 90 /-45]_{6}$ from a $[0 /+45 / 90 /-45]_{3 S}$, and detect an error in the form of a single misoriented ply: the 12 th ply in $[0 / 90 /+45 /-45]_{3 s}$ mistakenly placed at $+45^{\circ}$ instead of at $-4^{\circ}$. Shear waves can therefore detect subtle but realistic layup anomalies in composite laminates. In this work, a model is developed for the propagation of shear waves through a laminate with arbitary ply orientation and sequence. Experimental results and model predictions are compared, and the model is used for identifying layup anomalies that are detectable with shear waves. Potential applications of this new method in nondestructive evaluation of composite laminates will be discussed. [Work supported by NSF I/U Center for NDE.]

\section{0:45}

1aPA10. Interfacial waves in a finitely strained layered elastic half-space. Dimitrios A. Sotiropoulos (Dept. of Eng. Sci., Tech. Univ. of Crete, Chania 73100, Greece) and Christoforos G. Sifniotopoulos (Northwestern Univ., Evanston, IL 60208)

Propagating and standing interfacial waves between a surface layer and an underlying half-space, both under finite strain, are examined. The media are compressible nonlinear elastic and homogeneously pre-strained with their principal axes of pre-strain aligned, one axis being normal to the planar interface. For arbitrary strain energy functions and propagation along a principal axis of pre-strain, the dispersion equation is obtained. A low-frequency wave speed is subsequently obtained in explicit form yielding nonpropagation parameter conditions which for a specific state of stress hold at any frequency. The high-frequency limit of the dispersion equation yields the secular equation for interfacial waves between two half-spaces. It is then found that equal-density compressible materials may allow propagation and that compressible materials with equal shear wave velocities parallel to the interface may filter interfacial waves, even under isotropic in-plane stretching. For an arbitrary layer thickness as compared to the wavelength, material and pre-strain parameter conditions are also derived for the existence of standing waves as solutions of the bifurcation equation, a limiting case of the dispersion equation. 
are the most important basic resonances. The dipole center of mass relax-

1aPA11. The range of existence of reflected homogeneous and surface waves in elastic orthotropic materials. Sudhakar Nair (Dept. of Mech., Mater., and Aerospace Eng., Illinois Inst. of Technol., Chicago, IL 60616) and Dimitrios A. Sotiropoulos (Tech. Univ. of Crete, Chania 73100, Greece)

Reflection of elastic waves from the free surface of orthotropic incompressible materials is examined under plane strain conditions in a material symmetry plane. Use of the physically allowable energy flux vectors in conjunction with the propagation condition and the generalized Snell's law, yields formulas for the range of existence of the different types of reflected waves with respect to the two elastic parameters: the material orientation of the free surface and the angle of incidence. It is the consideration of the energy flux vectors that, in general, introduces the second elastic parameter and enlarges the range for the existence of either two homogeneous shear plane waves or one homogeneous and one supersonic surface wave. In the special case of transverse isotropy, the above formalas yield a homogeneous shear wave and a surface wave which diminishes when the angle of incidence is $45^{\circ}$, the wave-number vector coincides with the energy flux vector, and there enters only one elastic parameter.

\section{1:15}

1aPA12. Sound absorption height and width limits for polymer glass transitions. Bruce Hartmann, Gilbert F. Lee, John D. Lee, and Jeffry J. Fedderly (Polymer Sci. Group, Naval Surface Warfare Ctr., 10901 New Hampshire Ave., Silver Spring, MD 20903)

At the glass transition of a polymer, the shear sound absorption per wavelength displays a relaxation covering many decades of frequency. Calculations were made of the peak height and half-width of this relaxation based on the Havriliak-Negami dispersion relation. These calculations are an extension of our earlier study of the complex modulus loss factor height and width [B. Hartmann, G. F. Lee, and J. D. Lee, J. Acoust. Soc. Am. 95, 226-233 (1994)]. It was found that height and width are not independent: A high peak has a narrow width while broadband absorption can only be achieved for low-peak heights. The calculation predicts that height times width is approximately constant, as expected for a relaxation for which the area under the curve is constant. These predictions are compared with published experimental data on various polymers, chiefly polyurethanes, and found to be in good agreement.

\section{1:30}

1aPA13. Dipole relaxation rates of mass-loaded spherical inclusions in elastic matrix materials. Kurt P. Scharnhorst (Naval Surface Warfare Ctr., Carderock Div., White Oak Detachment, 10901 New Hampshire Ave., Silver Spring, MD 20903-5640)

It is necessary to be aware of the rise times of dominant resonances when tone bursts or wide bandwidth pulses are used to test the acoustic response characteristics of materials containing resonant inclusions. In practice the monopole of a void and the dipole of a mass-loaded inclusion ation rate of a mass-loaded, rigid spherical inclusions in an elastic matrix material is analyzed. Inclusion to matrix mass density ratios ranging from one to eight and matrix shear stiffnesses ranging over more than an order of magnitude are considered. It is found that the rise time of center of mass oscillations is much longer than that of cavity oscillations in the case of heavily mass-loaded inclusions in rigid matrix materials. In soft materials, the reverse is true. In terms of the period of oscillation at resonance, the relaxation time of the rigid dipole is less than half a period long. This rate may be viewed as the lower bound on the relaxation rates of mass-loaded viscoelastic inclusions.

\section{1:45}

1aPA14. Local temporal variance of Wigner's distribution. David H. Hughes (Code 7132, Naval Res. Lab., Washington, DC 20375-5000)

Lamb wave resonances on submerged spherical shell are analyzed via the local temporal variance of the signal Wigner distribution. The local resonance widths are oblained by approximating the local resonance contribution to the farfield backscattering form function of a particular Lamb wave as a resonance of the single Breit-Wigner type. The half-width of the resonance is approximately proportional to the inverse of the square root of the local temporal variance evaluated at resonance. This discrete distribution is compared against the continuous half-width distribution as rendered by the ray acoustic approximation for the particular Lamb wave under study. [Work supported by ONR.]

\section{2:00}

1aPA15. Borehole acoustic waveform decomposition and analysis via spectral estimation. Michael P. Ekstrom (Schlumberger Austin Res., 8311 North RR620, Austin, TX 78720-0015) and C. J. Randall (SciComp. Inc., Austin, TX 78731)

The response of an acoustic logging tool in a fluid-filled borehole is quite complex, being composed of multiple interferring components. Each component has unique velocity dispersion and attenuation characteristics which are related to properties of the formation surrounding the borehole. Robust and accurate methods to decompose the borehole signals and to analyze the components have been the subjects of much interest. In this paper, a new hybrid spectral estimator is introduced to estimate the velocity dispersion and attenuation of space-time wave fields from borehole array sensor data. This high-resolution estimator is based on an eigensystem decomposition of a matrix pencil, and provides an enhanced statistical performance while avoiding the severe ill conditioning of the classical Prony's method [Lang et al., Geophysics 52, 530-544 (1987)]. This improvement in spectral resolution allows the estimated wave-numberfrequency spectrum to be decomposed into its constitutive parts for subsequent individual analysis and wave field reconstruction. These estimation and decomposition procedures are mechanized in a set of MATLAB routines, and will be first validated by processing model waveforms for a homogeneous formation, then demonstrated with a variety of both model and field-acquired data [C. J. Randall, J. Acoust. Soc. Am. 90. 1620-1631 (1991)]. 


\title{
Session 1pAO
}

\author{
Acoustical Oceanography and Underwater Acoustics: Acoustic Interactions with Internal Waves \\ in Shallow Water II
}

\author{
Kevin B. Smith, Chair \\ Department of Physics, Naval Postgraduate School, Code PH/SK, Monterey, California 93943
}

Chair's Introduction-1:00

Invited Papers

1:05

1pAO1. The effect of internal waves on rays that turn sharply in deep and shallow water. Frank S. Henyey, Terry E. Ewart, Stephen A. Reynolds (Appl. Phys. Lab., Univ. of Washington, 1013 NE 40th St., Seattle, WA 98105), and Charles Macaskill (Univ. of Sydney, NSW, Australia)

Conditions for which an acoustic ray turns in a strong thermocline occur in both shallow and deep water. Internal wave induced fluctuations in the acoustic propagation are expected to be particularly large for such rays. A shallow-water example is the Synthetic Aperture Sonar experiment of summer, 1996, modeled with historical data for the site. A deep-water example is the upper path at the MATE experiment done a number of years ago. Conditions of validity of the Markov approximation are unlikely to hold under such conditions. In particular, the range derivative of the correlation length tangent to the ray greatly exceeds unity for the MATE upper path. Other approximations may also be inaccurate. A program to investigale standard approximation schemes for the MATE upper path has begun, whose purpose is to try to improve theories for such conditions, and to serve as a comparison case for the shallow-water experiment. Various statistical quantities are to be calculated in simulations, and compared to the same quantity calculated with standard approximations.

\section{1:35}

1pAO2. Modal sensitivity of acoustic interactions with internal waves in shallow water. Ji-Xun Zhou and Xue-Zhen Zhang (School of Mech. Eng., Georgia Inst. of Technol., Atlanta, GA 30332)

Analyses of individual acoustic mode interactions with internal waves should permit both qualitative and quantitative understanding of the influences of internal waves on multimode signals in shallow water. In this report, some experimental results taken in the Yellow Sea during the summer with strong thermoclines, will be reported. Numerical simulations will be used to analyze the potential internal wave interactions suggested by these data. (a) A multimode signal obtained at a single hydrophone and the first acoustic mode obtained by a mode filter array, both from a fixed $442-\mathrm{Hz} \mathrm{cw}$ source, exhibit very different fluctuation characteristics. (b) The transmission loss (TL) measured with explosive sources indicates a strong source/receiver depth dependence. (c) TL is, generally, anisotropic and can be abnormally large over some frequency range. It has been shown that the acoustic mode-coupling induced by internal waves can be an important loss mechanism for sound propagation during the summer [Zhou et al., J. Acoust. Soc. Am. 90, 2042-2054 (1991)]. The observed fluctuations can be explained in terms of the propagation characteristics of individual modes to the presence of internal waves. In so far as the mode-coupling and both the intensity and arrival time fluctuations are concerned, certain acoustic modes are more sensitive to internal waves than others. The characteristics of acoustic-internal wave interaction strongly depend on signal frequency, thermocline profile, seabottom properties, source/receiver depths and distance. [Work supported by ONR.]

1pAO3. Modeling the temporal coherence of pulse propagation in shallow water with internal wave and rough boundary scattering. F. D. Tappert (Appl. Marine Phys., Univ. of Miami, RSMAS, 4600 Rickenbacker Cswy., Miami, FL 33149)

For fixed source and receiver geometry, the broadband UMPE ocean acoustic model is used to make Monte Carlo predictions of pulse propagation in shallow water with internal wave and rough boundary scartering. The internal waves are modeled with a broad spectrum of excitation, and evolve slowly in geophysical time $t_{g}$ according to their intrinsic dynamics. For each realization of a field of internal wave fluctuations, assumed frozen at time $t_{g}$, a time series of complex demodulates of the received acoustic signal is computed by Fourier synthesis. The square modulus of this function yields the intensity envelope time series, $A(t)$, and many peaks of $A(t)$ correspond to ray arrivals and travel times that are spread by scattering. By advancing $t_{g}$ and repeating the pulse transmission simulation, the function $A\left(t, t_{g}\right)$ is computed and displayed as a "waterfall" plot. Examination of these plots gives quantitative information about the temporal stability and coherence of multipath arrivals in shallow water. Examples are presented in three distinct geographical areas: Florida Strait, New Jersey Shelf, and the Yellow Sea. [Work supported by ONR.] 
1pA04. Evidence for coastal generation of internal tides from deep-water acoustic observations. Stanley M. Flatté and Charles Bracher (Dept. of Phys., Univ. of Califomia, Santa Cruz, CA 95064)

In the summer of 1989 a 1000-km acoustic experiment in the Pacific showed a semidiumal variation in pulse travel time that was coherent in depth and varied systematically across timefronts. The effect of a single-plane-wave, gravest mode, M2, internal tide has been examined by ray tracing and has been found to show the same characteristic variation if the internal tide is coming from the direction of the Gulf of Alaska. In the process of fitting this specific acoustic data, an analysis technique for low-mode internal tides has been developed that can be applied generally to any acoustic tomography experiment.

3:05-3:20 Break

\section{Contributed Papers}

1pAO5. Mode coupling and other effects of modeled propagation through continental-shelf internal solitary waves. James C. Preisig and Timothy F. Duda (Appl. Ocean Phys. and Eng. Dept., Woods Hole Oceanogr. Inst., Woods Hole. MA 02543)

Acoustic propagation through a continental-shelf waveguide containing internal solitary waves has been modeled with the finite-element parabolic equation (FEPE). The waveguide has two essentially mixed layers separated by a gradient layer, with a homogeneous, lossy bottom. Solitary waves (pycnocline depressions) of many sizes and shapes are included, not all of which fit the dispersion relation. Including both physical and unphysical waves aids interpretation of the forward-scattering (mode coupling) mechanism. Solitary waves of horizontal scale length 50 to $150 \mathrm{~m}$ cause significant mode coupling. Longer and shorter solitons give weaker coupling. For $\mathrm{cw}$ signals $(400 \mathrm{~Hz})$, the results of a sudden approximation model for a single soliton are compared to those generated with the FEPE model and provide some insight into the mode coupling behavior. The results for a broadband pulse (e.g., 400- to $525-\mathrm{Hz}$ band) show a firstorder difference in waveform shape after propagation through a soliton when compared to the result with no soliton. This leads to the conclusion that received signal predictability based upon perturbation of layered-only propagation is problematic in the presence of the waves. [Work supported by ONR.]

\section{3:35}

1pAO6. Modeling backscatter from a shallow-water soliton: Relationship to the anomalous resonance effect observed in Yellow Sea data. Stanley A. Chin-Bing and David B. King (Naval Res. Lab.. Stennis Space Center, MS 39529-5004)

Computer simulations have confirmed that large amplitude shallowwater internal waves (solitons) can effect transmission loss. Zhou et al. [J. Acoust. Soc. Am. 82, 287-292 (1987)] first hypothesized that solitons caused the anomalous transmission loss observed in acoustic data taken in the Yellow Sea. Others [Chin-Bing et al.. Math. Model. Sci. Comput. 4, (1994); King ef al., Theoret. Comput. Acoust. 2, 793-807 (1994)] confirmed via computer simulations that interactions of acoustic energy with shallow-water solitons resulted in coupling between the lower-order propagation modes and the very lossy, higher-order modes. Furthermore, a single soliton packet could transfer sufficient acoustic energy from lowerorder modes to higher-order bottom-attenuated modes to produce the anomalous transmission loss effects observed in the Zhou data. Recently this simulation study has been extended to include a wave-number analysis of the backscattered field from the soliton. Examples of the backscantered field from the soliton will be presented that include the frequency interval where the forward-field acoustic mode conversion (and corresponding anomalous transmission loss) occur. [Work supported by ONR/NRL and by a Federal High Performance Computing DoD grant.]
3:50

1pA 07. Acoustic mode coupling induced by internal wave fields in shallow water. Steven Finette, Dirk Tielbuerger,a) and Stephen Wolf (Acoust. Div., Naval Res. Lab., Washington, DC 20375)

The problem of the propagation of acoustic waves in a stochastic ocean waveguide, for which the sound-speed variability within the water column is treated explicitly as a random variable, is addressed. The sound speed is composed of a deterministic, time-independent profile and two timedependent stochastic components representing a (linear) background Garrett-Munk internal wave field and (nonlinear) internal wave soliton packets. A high-angle elastic parabolic equation method is used to compute single-frequency realizations of the pressure field using this representation of the sound-speed fluctuations. Transmission loss and scintillation index measures are estimated for both the full field and its modal decomposition at various ranges from the acoustic source, for different source depths and for both flat and sloping bottoms. These measures are incorporated in the analysis of acoustic modal coupling induced by the internal wave fields as a function of range: results support a recent prediction [D. Creamer, submitted to J. Acoust. Soc. Am.] that the scintillation index increases exponentially with range due to the competition between mode coupling and mode stripping found in shallow water waveguides. ${ }^{\text {a) }}$ Permanent address: Forschungsanstalt der Bundeswehr für Wasserschall-und Geophysik, Klausdorfer Weg 2-24, 24148 Kiel, Germany.

\section{4:05}

1pA08. Modeling of high-frequency pulse propagation in the Yellow Sea with internal wave and rough boundary scattering. $X$. Tang. $F$. D. Tappert, and H. A. DeFerrari (Appl. Marine Phys., Univ. Miami, RSMAS, 4600 Rickenbacker Cswy., Miami, FL 33149)

The broadband UMPE ocean acoustic model is used to simulate highfrequency $(3 \mathrm{kHz})$ pulse propagation in the Yellow Sea. Internal waves are known to be active in the summer, and are modeled with a broad spectrum of excitation. Due to the existence of a strong thermocline in the upper part of the water column, the signal at a distant $(5 \mathrm{~km})$ receiver exhibits a characteristic multipath structure according to the geometric configuration of source and receiver-whether above and/or below the thermocline. When internal wave fluctuations are introduced, the simulated waveforms are altered and agree better with the experimental observation made in the summer of 1993 [R. Zhang, J. Acoust. Soc. Am. 97, 3368(A) (I995)]. Both rough surface and rough water-sediment interface scattering are included in the model in the belief that their effects cannot, in general, be separated from the volume scattering by internal waves. A 10-h time history of repeated pulse transmissions simulated in accordance with the recorded ocean temperafure fluctuation is computed and displayed, and illustates the temporal instability of shallow-water propagation. [Work supported by ONR.] 
new $\mathrm{PE}$ is used to model propagation of an acoustic field through an ocean with fluctuating sound-speed profiles caused by internal waves and other range dependent oceanographic properties. The effects of these new terms and the circumstances under which they are expected to be most important are discussed, with special emphasis placed on global-scale propagation.

4:35-4:40 Break

4:40-5:25

\section{PANEL DISCUSSION:}

Panel Moderator: Kevin B. Smith

Panel Members: John R. Apel, Terry E. Ewart, Stanley M. Flatte, Frank S. Henyey, James F. Lynch, Albert J. Plueddemann, Frederick D. Tappert, Ji-Xun Zhou

\title{
Session 1pEA
}

\section{Engineering Acoustics: Recent Trends in Loudspeakers}

\author{
John L. Butler, Cochair \\ Image Acoustics, Inc., 97 Elm Street, Cohasset, Massachusetts 02025 \\ Stephen C. Butler, Cochair \\ Naval Undersea Warfare Center, Code 2131, New London, Connecticut 06320
}

Chair's Introduction-2:00

\section{Invited Papers}

1pEA1. New developments in loudspeaker materials. Michael A. Klasco (Menlo Scientific, Ltd., 39 Menlo Pl., Berkeley, CA 94707)

Perhaps the drive behind a number of important new materials developments for speakers was the compact disc. The CD brought wide dynamic range with extended bass response into the home and car and "digital-ready speakers" have become more than just a marketing pitch. How are speaker engineers increasing excursion, thermal power handling, and maintaining performance characteristics at higher sound levels, while improving reliability? Specific solutions such as carbon fiber and Kevlar woven and nonwoven composite cones, new cone forming technologies. injection molded adhesiveless suspension surrounds, thermally conductive adhesives, thermally (but nonelectrically) conductive voice coil formers, high-temperature voice coil wire insulation and adhesives, a new magnetic geometry for high-excursion linear travel, ferrofluids for woofers, high-heat emmisivity plating techniques, and other fabriction and materials solutions will be briefly discussed. Advances in materials extend to enclosure materials, and a brief survey of developments in this related field will be mentioned. Additionally, test and measurement procedures to objectively quantify these enhancements will be touched upon.

1pEA2. Time-frequency display of loudspeakers and electroacoustic impulse response data using cycle-octave wavelet transforms. D. B. (Don) Keele, Jr. (DBK Assoc., 536 W. Mishawaka Rd., Elkhart, IN 46517)

A cycle-octave time-frequency display is created by plotting the magnitude of the wavelet transform, using a Morlet complex Gaussian wavelet, on a log-frequency scale versus time in number of cycles of the wavelet's center frequency. This type of display is quite well suited for plotting the decay response of wideband systems, such as the impulse response of a loudspeaker or room, because the time scale is long at low frequencies and short at high frequencies. If the response of typical filters are poltted on this type of display, the resultant 3-D responses are independent of the filter's center frequency, i.e., the decay response shape of a particular filter remains the same as it's center frequency is shifted up and down in log frequency. It can be shown that the wavelet transform of a system's impulse response is equivalent to an aggregate of the tone-burst responses of the system evaluated at equal-percentage-spaced frequencies, where the appropriately scaled and time-reversed mother wavelet acts as the tone-burst excitation signal. 
1pEA3. Recent advances in computer-aided design of loudspeakers. Vance Dickason (Vance Dickason Consulting, $333 \mathrm{~S}$. State St., Ste. 152, Lake Oswego, OR 97035)

Although successful CAE software that allows the accurate simulation and development of loudspeaker enclosures and crossover networks has been available for professional design work for several years, a number of loudspeaker industry practitioners still persist in using traditional methods of "cut and try." This presentation outlines a computer-aided loudspeaker development case study of a commercial three-way loudspeaker using a vented enclosure and a crossover network that incorporated fourth-order acoustic slopes. Measurement of the resulting prototype showed close agreement, within $0.5-1 \mathrm{~dB}$, with the computer simulation. Time required for the development of the fairly complex crossover network determined for this loudspeaker was less than $1.5 \mathrm{~h}$, substantially less than what is generally expected of "cut and try" methods. Key to the resulting accuracy using this type of computer software is the correct portrayal of interdriver time delay and driver phase. Driver phase for this project was derived from the driver magnitude using a highly sophisticated phase calculation methodology, as opposed to being directly measured with a two-port analyzer. Other criteria responsible for the success of the loudspeaker modeling portrayed in this case study are the ability to predetermine the group delay performance of the enclosure design, and the ability to examine crossover network transfer function during the design process.

3:15

1pEA4. Time Align® loudspeaker crossovers. Edward M. Long (E. M. Long Assoc., 4107 Oakmore Rd., Oakland, CA 94602)

A Time Align ${ }^{\circledR}$ loudspeaker system must produce an acoustical output at the listening position such that the fundamental and overtones of a complex, transient signal have the same relationships that they have at the electrical input to the system. To qualify for the use of the Time Align ${ }^{\circledR}$ trademark, the natural time offset between the acoustical outputs of the loudspeaker drivers must be corrected. This time offset is caused by the natural low-pass filter characteristics of the drivers and the electrical crossover filters. The time offsets between the acoustical output of each of the drivers must be corrected and the electrical crossover filters must be designed to produce an acoustical output from adjacent drivers that is $6 \mathrm{~dB}$ down from its passband level. When the coherent acoustical outputs of adjacent drivers are combined, the result is a uniform acoustical output. The polarity of the acoustical outputs is also affected by the crossovers; this is also a major consideration in the design of a Time Align® crossover. A Time Align® loudspeaker system must be able to produce acoustical square waves and have a short impulse response. Time Align® design techniques are shown by practical examples.

1pEA5. A new family of rotary loudspeaker transducers. Thomas J. Danley (Quantum Sound, Inc., 305 Era Dr., Northbrook, IL 60062)

A technical overview will be provided for a new electromagnetic transducer concept that employs rotary motion rather than rectilinear piston motion. The differences in the physical rules that govern the distribution of force and mass in the rotary and rectilinear systems will be explored. These relationships have permitted the construction of a new category of transducer which offers solutions to some of the seemingly insurmountable design constraints inherent in conventional coil/cone driver systems. One of the transducers examined is a high-output, full-range ( $40 \mathrm{~Hz}$ to $20 \mathrm{kHz}$ ) rotary loudspeaker which produces low-distortion, minimumphase acoustic output with a radiation pattern resembling that of a line source.

\section{3:55}

1pEA6. Design considerations for an application-specific loudspeaker. James E. Mitchell (Frazier Div., Sound-Craft Systems, Inc., Rt. 3, Box 319, Morrilton, AR 72110)

The design of a proprietary loudspeaker for use in IMAX theaters posed some unusual problems while offering a unique opportunity: that of optimizing a loudspeaker design for a predetermined and relatively narrowly defined set of acoustic, geometric, and mechanical conditions. Requirements unique to the application, a specialty cinema format which employs six discrete audio signal channels and makes substantial demands on sound system bandwidth and acoustic output, are explained. Methods used to arrive at the final loudspeaker design are presented. Sound system coverage prediction software was utilized in a novel fashion, significantly reducing the time required to complete the development of the loudspeaker. Investigation of IMAX theater geometries indicated that a vertically asymmetric radiation pattern was highly desirable. Optimum loudspeaker directivity criteria were established, and horns were developed with these criteria in mind. Testing of prototypes indicated that the criteria had been substantially met. Predictive work was verified with in situ testing of prototype systems.

\section{Contributed Papers}

\section{$4: 15$}

1pEA7. Neodymium iron boron and professional audio loudspeakers. Daniel M. Warren (Peavey Electronics Corp., 711 A St., Meridian, MS 39302)

Neodymium iron boron, a high reminence, high coercivity permanent magnet material, has been in use in the audio industry for several years in the form of small (approximately 1-cm-diam) disks in high-performance microphones. Dropping prices and continuing enhancements in material properties, reducing thermal demagnetization and increasing residual mag- netic flux density, have made neodymium more attractive for use in professional audio loudspeakers, where magnent size can reach 4 in. in diameter and almost $\frac{1}{2}$ in. thick. While still considerably more expensive than the more commonly used ferrite ceramic magnets, neodymium magnet structures can be smaller and have a higher flux density than can be practically attined with ceramics. A specific example of loudspeaker motor structure design using neodymium-the Architectural Acoustics Neo Series Acoustical Components from Peavey Electronics-will be presented. Acoustical implications of high-force loudspeakers on enclosure design will also be discussed. [Work supported by Peavey Electronics Corporation.] 
1pEA8. Near-field and far-field measurments of a ribbon tweeter/midrange. Alexander L. Butler and John L. Butler (Image Acoustics, Inc., 97 Elm St.. Cohasset, MA 02025)

It is common practice to make near-field pressure measurements on woofers as a means for avoiding low-frequency reflections from surroundings which can occur during far-field measurements. There is also a problem with making far-field measurements on ribbon tweeters. This is due to the long length of the ribbon compared to the wavelength which results in requirement of a very distant far-field microphone. As a result of this measurement problem, a technique has been developed for near-field measurements of ribbon tweeters. This technique is based on the Helmholtz integral equation evaluated on the axis of the tweeter. In the case of a rigid baffle only the pressure gradient measurements are needed over the surface of the ribbon. This paper will present the results of measurements of the pressure gradient as well as the pressure distribution in the vicinity of the ribbon and present a simple technique for predicting the far-field from these near-field measurements.

\title{
Session 1pNS
}

\section{Noise: Overview of Boundary and Finite Element Methods in Acoustics}

\author{
Frank H. Brittain, Chair \\ Bechtel Corporation, 50 Beale Street, San Francisco, California 94105
}

Chair's Introduction-1:30

Invited Papers

\section{1:35}

1pNS1. Practical considerations in the use of the boundary element method. Kenneth A. Cunefare (George W. Woodnuff School of Mech. Eng., Georgia Inst. of Technol., Atlanta, GA 30332-0405)

The use of the acoustic boundary element method (BEM) is gaining widespread acceptance in industrial applications. This paper will present practical considerations for successful application of the BEM. The major issues which require attention for accurate and reliable modeling of a particular structure, whether existing or under design, include proper mesh selection, geometric modeling, the so-called "nonuniqueness" problem and the methods for its solution and intergration with other modeling programs (e.g., coupled FEM/BEM approaches). Mesh selection relates to the choice of element interpolation order and integration order. Geometric modeling issues include determination of the level of detail required for accurate modeling. The nonuniqueness issue addresses the well-known failure of the unmodified acoustic BEM at certain discrete frequencies. Program integration relates to some potential difficulties that could arise when coupling FEM and BEM programs. Finally, the paper will review some of the particular applications of the acoustic BEM.

\section{2:05}

1pNS2. Finite element applications in acoustics. Walter Eversman (Mech. and Aerospace Eng. and Eng. Mechanics, Univ. of Missouri-Rolla, Rolla, MO 65401)

A review of finite element methods in acoustics is presented. General concepts of FEM such as weighted residual and variational formulations, element types and interpolation within elements, the assembly of elements, continuity of the solution at element boundaries, requirements placed on FEM meshes by high frequencies, the imposition of boundary conditions on rigid, flexible, and absorbing surfaces, the representation of sources, and the approximation of far-field radiation conditions are discussed. The application of FEM to several types of problems in acousties is demonstrated by reference to published results. Specific examples include: (1) interior problems such as resonant frequencies and forced response in enclosures such as car bodies, aircraft cabins, auditoria, and combustors; (2) sound propagation in moving media in ducts and propagation and radiation in and around aircraft turbofan engines; (3) structure and acoustic interatction such as transmission of sound through aircraft fuselages; (4) acoustic radiation problems which are related to (2) and (3). Emphasis is placed on the physical content of the various problems, mesh generation, the choice of elements, solution procedures, accuracy, and computer resource requirements.

\section{2:35}

1pNS3. Developing acoustic models for boundary and finite element methods. James T. Browell (Software Support, Automated Anal. Corp., 2805 S. Industrial, Ste. 100, Ann Arbor, MI 48104)

The software needed to develop meshes for acoustic boundary element methods (BEMs) and finite element methods (FEMs) are the ones commercially available for structural finite element codes. However, creating a mesh is only the first step to acoustic model development. The analyst must also consider meshing guidelines required and simplifying assumptions allowed by the various solution methods. The density of acoustic analysis meshes is primarily defined by the geometry of the domain boundary and the maximum frequency of the analysis. Accurately describing the distribution of boundary conditions and coupling interfaces also plays a role. While BEMs have distinct modeling advantages over FEMs, BEMs have specialized modeling requirements. In addition, BEM requires the creation of data recovery meshes to display results, because the surface of the model is not usually the point of interest. 
Simplifying assumptions are made in acoustic analyses to decrease the time for model development. For example, symmetry is used to simplify a problem. In addition, assumptions are made to simulate fully reflective or pressure release infinite planes, such as highways or ponds, sources are used to represent excitation boundary conditions in the far field, and acoustic damping is considered as surface impedance or complex speed of sound.

3:05

1pNS4. Fundamental issues of accuracy in boundary element analysis of acoustic problems. J. Trevelyan (Computational Mech. BEASY, Ashurst Lodge, Ashurst, Southampton SO40 7AA, UK)

The boundary element method (BEM) has become well established as a primary technique for the computer simulation of acoustic fields. Its ability to provide accurate predictions of sound-pressure levels in a variety of media, including those extending to "infinity," has led to a growth in the popularity of the method. Further, recent advances in acoustic diagnostic analysis, which is an extension of the traditional BEM approach, have added to the capabilities of this type of software simulation. It is important when using numerical simulation methods not to forget the potential sources of error which may be caused by improper usage of software systems. This paper discusses some of the fundamental modeling requirements of a good boundary element model, showing how the results can degrade when these requirements are violated.

3:35-3:50 Break

\section{Contributed Papers}

\section{3:50}

1pNS5. Numerical methods for gas turbine silencer design. Ramani Ramakrishnan and Eric D. Wilson (Vibron Ltd., 1720 Meyerside Dr., Mississauga, ON L5T 1A3, Canada)

Gas turbines are becoming common power producers in small to medium scale power plants. However, the environmental noise impact due to the proximity of these plants to urban centers has become a critical concem and large amounts of silencing are required. Passive and/or reactive elements are commonly used to silence the noise propagating from the turbine exhaust stack. Two factors, namely temperature and low-frequency dominance of the sound spectrum, must be properly accounted for in the silencer design. Simple conventional designs must include the effects of the above two parameters so that appropriate modifications can be incorporated to provide the necessary insertion loss. In this paper, performance evaluation of a passive silencer as well as a reactive silencer using numerical methods will be presented. Finite-element methods (FEM) have been successfully used to predict the performance of passive silencers. Details of the FEM procedure and the necessary temperature modifications will be highlighted. Boundary element methods (BEM) were applied to evaluate the performance of a reactive silencer. Simple tests to verify the results of the multidomain BEM methods were conducted. The results of the BEM procedure and the temperature modifications will also be presented in this paper.

\section{4:05}

1pNS6. Improvement of sound quality through accurate acoustic diagnostic boundary element analysis. J. Trevelyan, R. A. Adey, and S. M. Niku (Computational Mech. BEASY, Ashurst Lodge, Ashurst, Southampton SO40 7AA, UK)

The boundary element method (BEM) has long been used effectively in the prediction of sound-pressure levels in acoustic cavities and surrounding vibrating bodies. While this is an important capability in the design of structures for noise constraints, it does not point the engineer to the appropriate remedial action if the noise is found to be excessive. This paper presents a discussion of "acoustic diagnostic analysis," which is an analytical extension to the BEM which traces the source of any noise to its root cause. The impact of a design change can be seen immediately through the use of sensitivity computations, which clearly show the most effective modifications which can be made for noise reduction. The technique works by calculating the contributions made by each portion of the surface area to the sound-pressure level at an internal point. This allows an accurate evaluation of the contribution made from each part of the panel to the noise experienced at that point. The paper discusses some issues involved in performing the boundary element analysis for sound-pressure level prediction and calculating the panel contributions accurately. The paper also describes recent extensions to the method which allow acoustic diagnostic analysis of multidomain problems, and also how more accuracy can be obtained for problems including sound absorbing materials. Both of these developments have important consequences, in particular in automotive noise reduction. A test example shows how the technique locates the structural panels which are the major contributors to a noise problem, and which are therefore the panels which offer the most noise reduction effect for a given remedial action.

\section{$4: 20$}

1pNS7. A numerical approach to scattering by many scatterers of various sizes. Matthias G. Imhof (MIT Earth Resources Lab., E34-370, 42 Carleton St., Cambridge, MA 02142-1324)

A numerical method is derived for solving wave fields in the presence of many scatterers with different sizes. Three different scattering regimes are distingished: $a \ll \lambda, a<\lambda$, and $a>\lambda$, where $\lambda$ denotes the wavelength and $a$ is the maximal diameter of the particular scatterer. In the case of $a \ll \lambda$, the Rayleigh approximation can be used where the scattered wave field hardly depends on the geometry of the scatterer. For $a>\lambda$ the scattered fields are calculated using MMP expansions [M. G. Imhof, J. Acoust. Soc. Am. 97, 754-763 (1995)], which yields the full waveform solution by evaluating the boundary conditions. Scatterers with $a<\lambda$ are too large for the Rayleigh approximation to hold or for the scattered field to be independent of the geometry. On the other hand, the scatterers are too small to justify the MMP approach. Thus, the scattered fields are expanded into a series of Hankel functions where only the first few orders are used. Their weights are found in the least-squares sense by Galerkin's method. As an example, results are presented for many elliptical scatterers of different sizes.

1pNS8. Acoustic radiation prediction of an engine block using a combined finite element method and boundary element method technique integrated with test data. Ralph Garcea (LMS N. America, 5455 Corporate Dr., Ste. 303, Troy, MI 48098)

The project discussed in this paper was undertaken to validate the acoustic radiation prediction of engine block noise using a combined FEM and BEM technique, integrated with experimental data. Using a strictly analytical technique, the acoustic results from a BEM analysis will be limited by the accuracy of the velocity boundary conditions generated from structural FEM data. Experimentally determined velocity boundary conditions are more accurate, but they may not be available at every node of the BEM mesh. A new tool has been developed that combines the calculated structural modes of vibration with a limited set of measurement data, to 
extrapolate velocity boundary conditions on the entire BEM model. A FEM technique is used for the structural analysis and an indirect BEM technique is used to predict noise at some distance from the engine block. Very good correlation is obtained with measured sound-pressure levels. The modal expansion procedure combines experimental and numerical data to provide more velocity boundary conditions for the BEM acoustic analysis. Incorporating the test data helps to define a more realistic excitation and damping in the model, leading to more accurate noise predictions.

1pNS9. Wave scattering using a hybrid MMP-FE technique. Matthias G. Imhof (MIT Earth Resources Lab., Cambridge, MA 02142-1324)

Among the methods amendable to solve acoustic or elastic scattering problems are the finite elements method (FEM) and multiple multipole (MMP) expansions [M. G. Imhof, J. Acoust. Soc. Am. 97, 754-763 (1995)]. Among the difficulties the FEM encounters are long calculation times. large memory requirements, and the need for absorbing boundary conditions. On the other hand, the FEM is perfectly suited for heterogeneous media because it automatically accounts for changes in material properties. In contrast. the computational cost of MMP expansions is independent of the source-receiver geometry. An unbounded domain poses no difficulty since the expansion functions account automatically for the radiation condition. MMP expansions often converge with very few terms and therefore reduce the computational effort. Unfortunately, expansion functions for heterogeneous media are difficult to find. Scattering problems with heterogeneous scatterers embedded in a homogeneous background are difficult (FEM) or impossible (MMP) to solve with either of these techniques alone. Therefore, the MMP expansions are coupled with the FE method. For a cylindrical scattering problem, the resulting solution is compared to the analytical one. Results for multiple inhomogeneous scatterers embedded in a homogeneous media are presented.

\section{5:05}

IpNS10. Finite element models for sound absorbing materials. J. Stuart Bolton and Yeon June Kang (1077 Ray W. Herrick Labs., School of Mech. Eng., Purdue Univ., West Lafayette, IN 47907-1077)

Many noise control treatments feature porous materials that are either fibrous or foamlike. Analytical methods are available to model the behavior of these materials in simple geometries: e.g., infinite planar or cylindrical geometries. Realistic noise control treatments, however, are of finite size, are often multilayered, and may have lextured surfaces. If treatments of that type are to be designed optimally, numerical models of the materials must be used. Note also that when a surface is nonlocally reacting, the sound fields within the treatment and in the adjacent space must be solved for simultaneously. For both these reasons, a number of finite element formulations have been developed to model sound absorbing materials. The first models treated the absorbing medium as an effective fluid, and were used to model extended reaction fibrous materials. More recently, finite element models for elastic porous materials, i.e., foams, that are based on the Biot theory have been developed. The main features and capabilities of these various finite element models will be discussed in this presentation. In addition, a number of examples of their use will be given: e.g., the optimal design of a foam wedge for sound absorption and transmission control.

\title{
Session 1pPA
}

\section{Physical Acoustics: General II: Ultrasonics}

\author{
Michael J. White, Cochair \\ CECER-LV-P, U.S. Army Construction Engineering Research Laboratory, P.O. Box 9005, Champaign, Illinois 61826-9005
}

Yuan-Liang Li, Cochair

Department of Electrical Engineering, University of Illinois, Urbana, Illinois 61801

\section{Contributed Papers}

\section{1:00}

1pPA1. An improved iterative Born technique for ultrasound mammography. Lujiang Liu and Shira L. Broschat (School of Elec. Eng. and Comput. Sci., Washington State Univ., Pullman, WA 99164-2752)

In ultrasound mammography the objective is to image tumors about 4 or $5 \mathrm{~mm}$ in size. Thus the resolution of the reconstructed object profile is critical. Because of the strong refraction caused by fat lobes in the breast, simple pulse echo methods are inadequate, and a full inverse imaging technique is needed. The iterative Bom method is an inverse technique that has been used in ultrasound imaging. However, the calculation cost is very high, and parallel computation is limited to the forward problem. In this work, a method is presented that improves the performance of the iterative Born approach, giving better resolution at less cost. The dependence between the incident source angle and the reconstructed object profile is explored, and an algorithm is presented that is realizable on a parallel computer for both forward and inverse calculations. Four single sources are evenly spaced around the object to be imaged and are used to reconstruct the object simultaneously; the results from the four separate sources are then averaged. The cost of this method is $20 \%$ less than the iterative Bom method. In addition, this method is robust to noise since averaging helps to cancel noise. [Work supported by NSF.]

\section{1:15}

1pPA2. Acoustic imaging of thin films or interfaces. John G. Harris (Theor. Appl. Mech., Univ. of Illinois at Urbana-Champaign, Urbana, IL 61801) and Gerry Wickham (Brunel Univ., Uxbridge, Middlesex UB8 3PH, UK)

The imaging of thin solid films or interfaces by a confocal arrangement of ultrasonic transducers is modeled in both a two-dimensional and a three-dimensional context. In the first model, an antiplane shear, focused beam is directed across an interface comprised of an array of cylinders, with contrasting material properties. Its periodicity is destroyed by removing cylinders arbitrarily. In the second, a thin solid film, having quite general mechanical properties, is immersed in a fluid and scanned by a three-dimensional acoustic beam. This last work is only partially completed. An approximate integral representation of wave fields scattered by these thin regions of heterogeneity in terms of a Green's function for the 
host media, and the stress and momentum polarizations averaged through the thickness of the film or interface is the starting point. The stress polarization measures the change in elastic properties and the momentum polarization that in inertial properties, both changes being with respect to the properties of the host media. The scattered waves are calculated by formulating and solving integral equations. Those received are estimated using a measurement model that mimics the receiving transducer. [Supported by the NSF, MSS 91-14547.]

1pPA3. Development of a portable, focused-beam ultrasonic scanner for the NDI of adhesively bonded aircraft fuselage skin structures. Thadd C. Patton and David K. Hsu (FAA-Ctr. for Aviation Systems Reliability, Iowa State Univ., Ames, IA 50011)

In keeping with the requirements of the air carrier maintenance community, a closed-cycle, water-coupled, focused-beam ultrasonic method for the NDI of adhesively bonded aircraft fuselage structures has been developed. This approach, known as the "dripless bubbler" technique is the combination of focused-beam immersion ultrasonics with protable ultrasonic scanners. Because a focused ultrasonic beam is used during the scan, the spatial resolution is much greater than that obtainable with conventional flat contact transducers. The improved spatial resolution is necessary for the detection of localized corrosion pits and surface roughness associated with active corrosion sites in aluminum skin structures. The dripless bubbler allows for the capability to ultrasonically scan the exterior of an aircraft over surface protrusions such as button-head rivets in any orientation without the problem of uncontained couplant water. Recently the dripless bubbler has been involved in a technology deployment and transition program as part of the FAA-National Aging Aircraft Research Program. The result of this technology transfer will be a fully functional prototype scanner incorporating the dripless bubbler technique for rapid, large area NDI of aircraft bonded structures. Results will be presented on the development history of the dripless bubbler along with its current design and verification process. [Work supported by FAA.]

\section{$1: 45$}

1pPA4. Optical detection of ultrasound with a high-power pulsed laser. Hidetoshi Nakano, Youichi Matsuda, Shoichirou Shin, and Satoshi Nagai (Natl. Res. Lab. of Metrol., 1-1-4, Umezono, Tsukuba-shi, Ibaraki 305, Japan)

An optical system has been developed for detection of laser-generated ultrasound on rough surfaces at a distance over $1 \mathrm{~m}$. A long pulse Nd:YAG laser is used to prove samples. The laser provides a light pulse of $60 \mu \mathrm{s}$ in duration and $60 \mathrm{~mJ}$ in energy with a single frequency. The scattered light from the sample is focused into a multimode fiber and introduced to the modified confocal Fabry-Perot interferometer which has a wave plate in the cavity. The wave plate gives the different optical lengths of the cavity depending on the beam polarization. By adjusting the difference of the optical lengths, the ultrasound signals detected from the orthogonally polarized beams can be out of phase. Taking the difference of the signals produces the valance output which is less sensitive to intensity modulations of the pulsed laser. Longitudinal and shear waves have been observed in several metallic samples without signal averaging.

\section{2:00}

1pPA5. Time reversal of ultrasonic waves in solids: Theory and experiments. Carsten Draeger, Didier Cassereau, and Mathias Fink (Lab. Ondes et Acoustique, Université Paris 7, E.S.P.C.I., 10 rue Vauquelin, 75231 Paris Cedex 05, France)

In previous works, the capacity of time-reversal mirtors (TRM) has been presented to optimize ultrasonic focusing on a pointlike source. However, the evaluation of the focusing quality has so far been limited to fluid media. In this paper, the first theoretical approach is presented to determine the capacity of the TRM to focus on a point situated in a homogeneous isotropic solid. $P$ and $S$ waves emitted by a pointlike source in the solid yield the generation of transmitted pressure waves in a fluid that limits the solid via a plane boundary. A TRM placed in the fluid behind the interface measures both incoming wavefronts and is hence able to reverse them one by one. The reversed pressure fields backpropagate to the interface, resulting in $P$ and $S$ waves in the solid. It is shown that, in spite of the losses at the interface, both kinds of waves focus at the location of the initial source. Numerical simulations are carried out, showing that the time reversal is more favorable using the $S$ waves than the $P$ waves, in terms of the width of the focal spot as well as in terms of displacement amplitude. The results are compared with experiments carried out with a laser source working in the ablation regime.

\section{2:15}

1pPA6. Harmonic generation measurements as a function of frequency in a carbon-fiber-epoxy-resin composite. Paul Elmore and M. A. Breazeale (Natl. Ctr. for Physical Acoust., Univ. of Mississippi, University, MS 38677)

Harmonic generation in a carbon-fiber-epoxy-resin composite has been measured as a function of frequency between 4.0 and $8.5 \mathrm{MHz}$. The amplitudes of both the fundamental and the generated second harmonics were measured absolutely with a capacitive receiver at room temperature. Phase velocities also were measured. Dispersion is seen in the conventional nonlinearity parameter, but not in the phase velocity. The composite sample is highly attenuative and may have additional nonlinear terms that are not yet accounted for theoretically. Since the conventional nonlinearity parameter is defined for a lossless solid, data interpertation is unclear at present.

\section{2:30}

1pPA7. Detailed observation of the complete fracture process of brittle carbon foam. L. C. Krysac and J. D. Maynard (Dept. of Phys., Penn State Univ., 104 Davey Lab., University Park, PA 16802)

Using wideband transducers, we have observed the acoustic signals produced by individual bond breaking events during the fracture of open cell brittle carbon. The complete fracture process, beginning with intermittent precursor bursts of small numbers of bonds breaking, and ending with the final rupturing catastrophic event, has been recorded with $20-\mathrm{MHz}$ pinducers and a 400-Megasample/s digital oscilloscope. The statistics of the precursors, etc., including the force applied at any time during the fracture process, have been acquired, related to the acoustic record, and compared with the predictions of the random fuse model for the fracture of brittle material. The role of dynamics and stress waves affecting the fracture of real materials, which is not accounted for in the random fuse model, will be discussed.

\section{$2: 45$}

1pPA8. Phase-insensitive homomorphic image processing for speckle reduction. Yan Chen, Shira L. Broschat, and Patrick J. Flynn (School of Elec. Eng. and Comput. Sci., Washington State Univ., Pullman, WA 99164-2752)

Speckle appears in all conventional ultrasound images and is caused by the use of a coherent transducer. Speckle is an undesirable property as it can mask small but perhaps diagnostically significant features. Speckle noise can be reduced using a phase-insensitive imaging technique to cancel the linear phase relationship between elements. However, with current devices this is hard to achieve because of the large memory and high sampling rate requirements for the 64 or more multiple channels used. To address the problems above, a hybrid method is introduced that combines the phase-insensitive technique with a phase-sensitive speckle reduction method. Several phase-correlated subimages ( 2 or 4 ) are formed using classical imaging techniques. Then nonlinear homomorphic processing is applied to destroy the phase relationship between these images. The homomorphic, phase-insensitive, and hybrid nonlinear processing method is developed and examined in this paper. Experiments with synthetic and real ultrasound imagery show that the proposed method improves the signalto-noise ratio in both lesion and cyst areas and preserves edge clarity. [Work supported by NSF.] 


\section{3:15}

1pPA9. Evaluation of source sound pressure of an ultrasonic transducer using schlieren visualization. Toru Tuziuti, Teruyuki Kozuka, and Hideto Mitome (Natl. Industrial Res. Inst. of Nagoya, 1-1 Hirate-cho, Kita-ku, Nagoya 462, Japan)

Source sound pressure has been evaluated without disturbing the sound field using an optical method of schlieren visualization. The intensity of schlieren light is described as a function of the Raman-Nath parameter $\nu$ and increases monotonously up to $\nu=2.405$. Experimental results showed that the light intensity on the sound beam axis changed with the applied voltage to the transducer in a similar manner to the above function. Then $\nu$ can be obtained numerically by substituting normalized experimental intensity for the function. Because the light passes through inhomogeneous sound field radiated from finite aperture, $\nu$ is affected by an integral optical effect. If the measuring position is fixed, this effect can be calculated theoretically in relation to the parameter just in front of the sound source defined as $\nu_{0}$. Hence the source sound pressure $P_{0}$ is estimated from $\nu_{0}$ using transducer size, wavelength of the light, and the refractive index of medium. The upper limit for $P_{0}$ of this method is $1 \times 10^{5} \mathrm{~Pa}$ in the present experiment.

\section{3:30}

1pPA10. Numerical study on particle velocity and sound pressure by circular flat transducers. Tohru Imamura (Natl. Res. Lab. of Metrol., 1-1-4, Umezono, Tsukuba, Ibaraki 305, Japan)

During years of investigation on the ultrasonic near field, only the sound pressure, namely, the spatial distribution of velocity potential, has been studied. In this report, particle velocity and acoustic impedance density of the ultrasonic field by circular flat transducers are derived and computed together with sound pressure. Sound pressure is proportional to the velocity potential of the ultrasonic field. Its particle velocity is the space differential of the velocity potential, and the acoustic impedance density is the quotient of the sound pressure by the particle velocity. On the axis of the transmitting circular flat transducer, the phase delay of the sound pressure has peculiar leaps. But, acoustic impedance density has constant leaps from $-\pi / 2$ to $\pi / 2$, where the amplitude is zero. The mean value over a receiving coaxial circular flat transducer is also computed changing the ratio of the radius (a) of the circular flat transducer to the wavelength $(\lambda)$ of the ultrasonic wave. Mean amplitudes of sound pressure, particle velocity, and acoustic impedance density are tabulated with the normalized distance $\left(z \lambda / a^{2}\right)$ in the computing precision of $0.1 \%$. The mean amplitude of the $z$ component of the particle velocity is always less than 1.0 and seems to be an appropriate response for the ultrasonic system of a pair of circular flat transducers.

\section{3:45}

1pPA11. A new approach to compute the resultant pressure of linear array. Jamal Assaad, Christian Bruneel, and Isabelle Looten (Dept. of OAE, IEMN, Valenciennes Univ., BP 311 Le Mont-Houy, 59304 Valenciennes, France)

In this paper, a new calculation scheme is proposed to compute the nearfield and the farfield directivity pattern of such arrays including interactions and without assumptions concerning the displacement field of the transducer radiating surface. For this purpose, using the finite element method (FEM), the farfield directivity pattern of a part of the array ( $2 Q$ +1 transducers) is computed using dipolar dampers and a previous described extrapolation algorithm [Assaad et al., J. Acoust. Soc. Am. 94, 562-573 (1993)]. This part consists of an active elementary transducer (electrically driven) mounted between $2 \mathrm{Q}$ passives (electrically grounded) neighboring transducers. Then, the radiation field of a finite phased and focused array ( $N$ elements) can be obtained by summing up the farfield directivity patterns of the $2 Q+1$ transducer sets weighted by the classical term used to take account of the geometrical and electrical phase shifts.
1pPA12. Response of a focused transducer facing a rigid reflector. Martin Manley and Nelson Hsu (Natl. Inst. of Standards and Technol. Rm. A 147, Bldg. 233, Gaithersburg, MD 20899)

The diffraction correction factor for a transducer first acting as a transmitter and then receiving the signal reflected off a rigid plane is useful in determining the system transfer function of a pulse-echo system. The determination of the diffraction correction factor is well understood for flat disk transducers, but is more complex for focused transducers. Recently, a method was derived [Chen et al., "Acoustic coupling from a focused transducer to a flat plate and back to the transducer," J. Acoust. Soc. Am. 95, 3049-3054 (1994)] for approximating the diffraction correction factor of a cylindrically focused transducer for the case when the rigid plane is close to the focal plane of the transducer. In this work, results are presented for cases when the rigid plane is not close to the focal plane of the reflector.

\section{4:15}

1pPA13. Implosion sound. Nai-chyuan Yen (Naval Res. Lab., Washington, DC 20375-5000)

An open end axisymmetrical cavity is used to study the generation of implosion sound. The cross section of the cavity's profile has a shape which will distort the wave front of a plane shock wave converging it into a single point. Hence, a spherical collapsing phenomenon can be approximately achieved when a shock wave propagates into the opening of this finite size artificial cavity. Through the shock and shock interference mechanism, a strong impulse therefore is generated at the focal point caused by the pressure difference between the inside and outside of the cavity. This highly localized impulse then is radiated out from the opening of the cavity as a sound projector. A prototype model of $0.5 \ell$ size has been constructed and tested in the laboratory. The preliminary experimental results indicated that this device can reach a peak sound pressure level of $146 \mathrm{~dB} / / 20 \mu \mathrm{Pa}$ in air and $185 \mathrm{~dB} / / 30 \mu \mathrm{Pa}$ in water, with an ambient pressure of 1 atmosphere ( $1 \mathrm{bar}$ ). The 50\% pulse width is about 20 and 30 $\mu \mathrm{s}$ for air and water, respectively. Analysis and application of this type of sound projector will be discussed.

\section{4:30}

1pPA14. Systematic measurements of duct end corrections in the Greenspan viscometer. Keith A. Gillis, Michael R. Moldover, and James B. Mehl (Natl. Inst. of Standands and Technol., Thermophys. Div., Gaithersburg, MD 20899)

A Greenspan viscometer is a Helmholtz resonator that has been optimized for viscosity measurements in gases. It consists of two chambers connected by a duct. The model, design considerations, and performance of such a device have been described elsewhere [Gillis et al., "Acoustic Greenspan viscometer for gases," submitted to Rev. Sci. Instrum.]. The model represented the effects of nonuniform flow near the ends of the duct as a complex acoustic impedance $\mathbf{Z}_{\text {end }}$, based on the numerical calculations by Mehl [J. Acoust. Soc. Am. 97, 3327 (1995) and unpublished calculations]. In this work, the "end corrections" were measured in a Greenspan viscometer with interchangeable ducts and a detector located in each chamber. This new design enabled a series of viscosity measurements to be performed with the same chambers and transducers, and with three different duct lengths and two duct diameters. The consistency between the experiments and the model will be presented. [Work supported by the Office of Naval Research.]

\section{4:45}

1pPA15. Numerical investigations of modified Greenspan viscometers. James B. Mehl (Natl. Inst. of Standards and Technol., Thermophysics Div., Gaithersburg, MD 20899, and Phys. Dept., Univ. of Delaware, Newark, DE 19711-2570)

Modified Greenspan viscometers are being studied with the objective of better understanding of inertial and dissipative duct-end effects for application to Greenspan viscometers. Greenspan viscometers are double 
Helmholtz resonators comprised of a circular duct coupling two concentric cylindrical chambers. Previous investigations [J. B. Mehl, J. Acoust. Soc. Am. 97, 3327 (1995)] of the acoustic fields and eigenfrequencies of viscometers with ducts terminating flush with the chamber ends have been extended to ducts which extend into the chambers a distance $D$. Numerically determined end effects are compared with analytic results for radiation from baffled and unbaffled open ducts. When $D=0$ the inertial end correction agrees well with the correction for a baffled open end; when $D$ is greater than a few duct diameters it approaches the value for an unbaffled termination. In contrast, the resistance of the duct end (defined for the limiting case of a small viscous penetration length) is not sensitive to $D$. The numerical model permits easy investigation of the effects of rounding and chamfering comers. [Work supported by the Office of Naval Research.]

\section{$5: 00$}

1pPA16. Sound velocity measurements of thin sheet materials at high temperatures. Youichi Matsuda, Hidetoshi Nakano, Shoichirou Shin, and Satoshi Nagai (Natl. Res. Lab. of Metrol., 1-1-4, Umezono, Tsukuba, Ibaraki 305, Japan)

Longitudinal sound velocities of thin sheet materials were determined between room temperature and $1000^{\circ} \mathrm{C}$. The velocities were calculated from sample thickness and round trip echo times. A micrometer or an ellipsometer was used for thickness measurement. A single optical pulse was extracted from a passively mode-locked Nd:YAG laser by using an optical switch. The optical pulse has 30-ps pulse duration and $10-\mathrm{mJ}$ output energy, which is focused on the sample surface to generate ultrasound. Ultrasound is detected with a Michelson interferometer having a frequency response up to $1 \mathrm{GHz}$. Since this system has no contact with the sample, it is suitable for acoustic measurements at high temperatures. Sound velocities of 304 stainless-steel sheets were measured at various temperatures in the range from RT to $1000^{\circ} \mathrm{C}$. It was shown that round trip echo time could be measured to an accuracy of $3 \%$ for the sample of $20 \mu \mathrm{m}$ thickness. Furthermore, several kinds of ceramic thin sheets were also examined.

\section{$5: 15$}

1pPA17. Networks for piezoceramic resonators. Arthur Ballato (US Army Res. Lab., AMSRL-PS, Fort Monmouth, NJ 07703-5601) and John Ballato (Rutgers Univ., New Brunswick, NJ 08855)

Equivalent circuits for characterizing piezoelectric resonators have been used for many years, and in a wide variety of applications. The future will see applications extended through the UHF band for ferroelectric ceramic thin-film resonant microstructures, e.g., for cellular radios. Such applications will require material and processing uniformity far greater than is currently achieved, and means for their verification. Piezoceramics occupy a difficult position, however, when it is required to obtain precise material parameters from broadband electrical measurements. This situation arises because of their particular attributes: high coupling and moderate loss. Highly accurate lumped equivalent networks for these frequencies have been developed for both canonical cases of thickness- and lateral-field excitation. Measurements on these circuits permit the extraction of the complex dielectric, piezoelectric, and elastic parameters of the ceramic material. This paper discusses both traditional and modem electrical network descriptions of piezoelectric resonators; and also treats how they are modified by the conditions of excitation and inclusion of loss mechanisms.

MONDAY EVENING, 27 NOVEMBER 1995

ST. LOUIS E, 7:00 TO 9:00 P.M

Session 1eID

Interdisciplinary: Tutorial Lecture on Overview of Musical Instrument Physics

\author{
Uwe J. Hansen, Chair \\ Department of Physics, Indiana State University, Terre Haute, Indiana 47809
}

Chair's Introduction-7:00

Invited Paper

$7: 05$

1eID1. Overview of musical instrument physics. Gabriel Weinreich (Randall Lab. of Phys., Univ. of Michigan, Ann Arbor, MI 48109-1120)

Most devices which can be used to "make sound," in the sense of converting frequencies in the range of human gestures to those within the audible band, are nonetheless not in use as musical instruments. What is required in addition is a large, but precise, control parameter space that makes an instrument "playable," that is capable of transmitting a complex musical message. Within this parameter space, the exact control of periodicity is especially important, particularly so in Westem music. After first exploring how the requirements of pitch stability, acoustic output capability, and sustaining power are satisfied in various instruments that vibrate freely after an initial excitation, the discussion will continue with the more complex physics of the bowed string and the blown pipe in their various embodiments. In many cases, it will turn out that instruments in actual use are not "engineering compromises" but, on the contrary, based on fortuitous cooperation among otherwise unrelated factors. The lecture will conclude with a brief discussion of the computer as a musical instrument. 
Session 2aAA

\title{
Architectural Acoustics: Residential Acoustics
}

\author{
Charles T. Moritz, Chair \\ Collaboration in Science and Technology, Inc., 15835 Park Ten Place, Suite 105, Houston, Texas 77084-5131
}

Chair's Introduction-8:30

\section{Invited Papers}

\begin{abstract}
8:35
2aAA1. Residential acoustics-An historical perspective. Ewart A. Wetherill (Paoletti Assoc., Inc., 40 Gold St., San Francisco, CA 94133)

A review of scientific and general writing on dwelling places confirms that disturbance of residents by both exterior and interior noises has been a major concern of urban living for more than 2000 years. Empirical experiments in reduction of intruding noise appear to have developed into traditional planning and building methods, such as separation of "active" from sleeping rooms and filling of floor joist cavities to enhance noise isolation. From a handful of references in the 19th century, the rate of publication of theoretical and experimental studies as well as nontechnical writing covering all aspects of acoustical intrusion increased dramatically in the early decades of the 20th century. These have led to international standardization of acceptability and measurement, and to regulation of acoustical performance in dwellings. Concurrent with this, however, the increase in types and locations of noise source--together with ways of reducing construction costs—have resulted in living environments in which both outdoor and neighbor noise may be worse than at any time in history.
\end{abstract}

9:05

2aAA2. Residential acoustics-A European perspective. Tor Kihlman (Dept. of Appl. Acoust., Chalmers Univ. of Technol., S-412 96 Göteborg, Sweden)

For a long time European building codes have included requirements on sound insulation between dwellings as well as noise levels from indoor and outdoor sources. Minimum requirements on sound insulation in finished buildings are based on the ISO documents 140 and 717. Typical minimum requirements lie in the range $R_{w}^{\prime} \geqslant 52-55 \mathrm{~dB}$ and $L_{n w}^{\prime} \leqslant 53-58 \mathrm{~dB}$. Today's trend is to extend the frequency range down to $50 \mathrm{~Hz}$ and to sharpen the minimum requirements somewhat, which creates some problems. In response to increasing demands from consumers, different classification systems for the sound insulation have been developed. The highest class demands insulation values up to $10 \mathrm{~dB}$ better than the legal requirements. A-weighted levels from building installations may not exceed 30-35 dB. To limit low-frequency ventilation noise $\mathrm{C}$-weighted levels are specified in addition to A-weighted levels. Traffic noise levels are based on $L_{e q}$ as a $L_{\mathrm{dn}}$ or as separate values for day and night. Typical demand is $L_{\mathrm{Aeq}}<55 \mathrm{~dB}$ outdoors and $30 \mathrm{~dB}$ indoors. In existing situations, these levels are often exceeded. Effort is now being spent in the European community to deal with this problem.

9:35

2aAA3. Acoustical defects in multifamily construction. John J. Van Houten and David L. Wieland (J. J. Van Houten and Assoc., Inc., Irvine, CA 92714)

Defects in multifamily construction have plagued the building industry in recent years. These defects are elements of the completed building which do not meet building code standards and/or may not conform to the architectural details or specifications. Additionally, defects in construction may involve contractual considerations and the homeowner complaint history. The identification of acoustical defects in multifamily construction requires a comprehensive review of the project documentation and field tests of a representative number of the floor-ceiling and/or party wall separations. The noise level of air handling equipment, elevator systems, and plumbing installations are also obtained. The findings of these tests are compared to noise insulation standards, established guidelines, and in some cases, home buyer expectations. Marketing information and disclosures conveyed at the time of sale are factors which may influence the evaluation of defects. Additional investigation usually involves destructive inspections of the actual assemblies, plumbing and equipment installations. These inspections are vital to the identification of recommended repairs. The various defects observed in multifamily construction within California will be indicated and the conflicting interests of homeowners, builders, and subcontractors will be discussed.

10:05

2aAA4. Acoustical and planning considerations for home theater and multichannel music playback systems. Thomas $\mathbf{R}$. Horrall (Acentech, 125 CambridgePark Dr., Cambridge, MA 02140)

Optimum multichannel sound playback in the home environment requires different room acoustical characteristics than traditional two channel playback. In particular, room surfaces should be acoustically more absorptive in order to avoid masking of later arriving reflections encoded in the software; and the availability of more than two channel playback permits such room design while preserving and enhancing the listener's sense of immersion in the sound field. Newer recorded five and six channel transmission systems 
employing a dedicated low-frequency effects channel require careful consideration of the type and placement of low-frequency loudspeakers. The optimum location and directional and other characteristics of loudspeakers intended for existing home theater audio formats are often considered different than those for music playback, but multichannel transmission formats for both video and music-only playback may impact these requirements. Layout requirements of the room, including screen size and placement, are reviewed in light of the coming availability of high resolution video formats such as the digital video disc (DVD). Finally, some measurements of acoustical performance in the author's home playback facility are presented.

\section{0:35-10:50 Break}

\section{Contributed Papers}

\section{0:50}

2aAA5. Measurements of airborne and impact sound through floors-A new project. Alf C. C. Warnock (lnst. for Res. in Construction, Natl. Res. Council of Canada, Montreal Rd., Ottawa, ON K1A 0R6, Canada)

A comprehensive project to study all aspects of sound transmission through floors has just been initiated at the National Research Council of Canada. As well as airborne sound transmission, measurements will include: impact sound transmission using a standard tapping machine, a special rubber ball, a walker, and a tire machine meeting the relevant Japanese standard. As well as these measurements on complete floor systems, all material properties will be measured to provide a complete characterization of the floor systems. Vibration measurements to determine modal characteristics and damping will also be made. These measurements will be made in the new floor facility at NRC which has been found to give more reliable results than the old facility, which is no longer in use. Floors in the new facility measure $3.8 \times 4.7 \mathrm{~m}$, a more realistic size than the 2.4 $\times 2.4 \mathrm{~m}$ floor in the obsolete facility. An outline of the project will be given. Initial measurements on wood joist floors to look at the effects of methods of attaching the floor and ceiling layers will be presented.

\section{1:05}

2aAA6. Acoustical characterization of straw bales as structural elements. Carl J. Mas and E. Carr Everbach (Swarthmore College, Dept. of Eng., 500 College Ave., Swarthmore, PA 19081)

For several thousand years, straw has been used mainly as bedding for horses and cattle. This inexpensive, thermally insulating, and environmentally renewable material has been used increasingly in the southwestern United States as a structural element in the construction of residential houses, storage facilities, and restaurants. An understanding of the acous- tical properties of straw bales is therefore required if this medium is to be used appropriately. The transmission loss (TL) of wheat and rye-grass straw bales was measured for bales placed in different configurations, and for the stucco-covered wall of a straw bale house. The TL for the straw bale house wall was $59.4 \mathrm{~dB}$ (A-weighted). The coefficient of acoustic absorption of the straw bales was also measured at 125, 250,500,1000, 2000 , and $4000 \mathrm{~Hz}$. [Work supported by an NSF PFF.]

\section{$11: 20$}

2aAA7. Acoustical behavior of a new lightweight partition made with gypsum board and cork. António Pedro O. Carvalho (Acoust. Lab., Dept. of Civil Eng., Faculty of Eng., Univ. of Porto, R. Bragas, P-4099 Porto Codex, Portugal)

The building construction development in Portugal has been changed by the gradual move to a general use of residential partitions made with increasingly light materials from the traditional heavy interior walls. A socioeconomic analysis of the Portuguese situation as it is concerned with the lodging policies and building construction industry, is briefly presented. An international comparison on this matter is shown using some economic parameters. The Portuguese situation is compared to 36 other countries. The main goal of this paper is to present a study of the acoustical behavior of lightweight partitions, especially those usually called "sandwich-type" and its experimental application to a new and very specific kind of partition made of gypsum board and cork, a traditional material in Portugal. This newly developed sandwich lightweight partition is presented and acoustically characterized. A new and simple mathematical model is presented to evaluate the sound isolation of this kind of partition. The results obtained in reverberant chamber tests are presented as well as the comparisons with the predicted values using the new model proposed. Different single and double wall types were tested giving STC values up to $44 \mathrm{~dB}$. [Work supported by LNEC and Univ. of Porto-Portugal.] 


\title{
Session 2aAO
}

\section{Acoustical Oceanography: Acoustic Inversion of Fish and Plankton Ensembles I}

\author{
Christopher Feuillade, Cochair \\ Naval Research Laboratory, Stennis Space Center; Mississippi 39529-5004
}

Timothy K. Stanton. Cochair

Woods Hole Oceanographic Institution, Department of Applied Ocean Phrsics and Engineering, Bigelow 201. Woods Hole, Massachusetts 02543

Chair's Introduction-8:55

Invited Papers

9:00

2aAO1. Acoustic visualization of zooplankton and micronekton patchiness in oceanic ecosystems. Charles $\mathrm{H}$. Greenc (Ocean Resources and Ecosystems Prog., Cornell Univ. Ihacil, VY 14853) and Peter H. Wiebe (Woods Hole Occanogr. Inst. Wonds Hole. MA 02543$\}$

Acoustic visualization combines the techniques of acoustic remole sensine and seientific data visualizalion. It provides scientists with the neans for interaclively exploring three-dimensional datal sets collected acoustically. In this presentation, lielel investigations will be presented in which acoustic visualization has provided unique insights into the three-dimensional patchiness of zooplanhton and miconekton distributions. The steps involved with survey design as well as data analysis and interpretalion will be discushed along with a video presentation. Details will be given of atlempts to derive quantilative, objective interences from irregualrly spated and temporally inconsistent data collecled during acoustic surveys of patchily distributed animals in an advective flow field. WWork supported by ONR. NSF, and NOAA.T

$9: 25$

2aAO2. Getting the most out of acoustic data: Linking acoustics with spatially explicit ecological models. Stephen B. Brandt (Great Lakes Crr., Buffalo Stale College. 130)( Elmwood Ave. Buffalo. NY' 1+222)

Acoustic data are rich in information but models or other data (e..... sound speed) need to be applied to the raw, time-dependent voltages to extract information on fish sizes, ahundances. and distribution. Information extraction is hierarchical in thal the more models (or assumptions) used, the more information obtained from the datat. Recent researcl combines spaltally explicit acoustic datia with other environmental measurements to provide a template for ecological modeling of tish feedling and growth. This modeling links the physical and biological structure of the habitat in al way that maintains the spatial inlegrily of the system. Datal visealizaltion. geographic information systems. and data animation are used to display model oulpul. The approach makes more full use of the spatial information inherent in acoustic data and can be used to predict lish growth and produclion. predalor-prey interactions. the polential success of species introductions, and the eflects of envirommental perturbations. [Work supported by NSF. Biological Oceanography.]

\section{9:50}

2aA03. Acouslic backscattering by complex zooplanklon assemblages on Georges Bank. Peler H. Wiebe (Depr. ol Bio!., Woods Hole Occanogr. Inst. Woods Hole. MA 02543). Tinotly K. Stanton (Woods Hole Oceanogr. Inst. Woods Hole. MA 025+31. and Charles H. Greene (Cornell Univ, lthaca, NY 14853-2701)

High-frequency $(420 \mathrm{kH}$ ) sound was used to study the volume hackscattering from plankion and micronekton over Georges Bank as part of a study designed to understand the relationship between volume backscaltering and the composition of ulse plankron. Volume backscattering levels at stratified sites were factors of 4 to 7 times higher than at a well mixed site. However. there was no significant ditference in MOCNESS sampled biomass between the locations and the regression between volume backscattering and total biovolume was not significant. The difference in volume backscattering is duc to differences in the acoustic scaltering properties of zooplankton taxa and the fact that the taxonomic composilion of the plankton differed between the sites. When laxal-specitic model predictions of acoustic backscattering cross section were used with fiekd size and abundance data to predicl measured volume hackscattering data. a highly significant relationship between observed and predicted volume backscattering was obtained. [Work supported by ONR. NSF, and NOAA.] 
2aAO4. Analysis of rather high-frequency sound echoes from ensembles of fish. C. S. Clay (Dept. of Geology and Geophys., Univ. of Wisconsin-Madison, 1215 W. Dayton St., Madison, WI 53706) and John K. Home (State Univ. of New York at Buffalo, Buffalo, NY 14222-1095)

At rather high frequencies, fish length/wavelength ratios are in the range 1 to 20 and the majority of scattered sound originates at the swimbladder. The sonar frequency, shape, and tilt of the swimbladder affect echo amplitudes. Echo amplitudes also depend on the position of a fish in the sonar beam. Echo emplitudes can be corrected for range and position in the sonar beam, but are still dependent on sonar frequency and swimbladder orientation relative to the soundwave front. Acoustic fish models were used to examine the dependence of echo amplitudes on frequency and swimbladder orientation [C. S. Clay and J. K. Home, J. Acoust. Soc. Am. 96, 1661-1669 (1994)]. Probability density functions (PDF) of echo amplitudes were computed for sets of fish orientation angles. The PDFs of single and ensernbles of fish were sensitive to sonar frequency and fish orientation. These PDFs are more interesting than those of Rayleigh and Rice and give additional data to characterize fish targets. [Work supported in part by the NSF (OCE-8817171, OCE-9115740) and Office of Naval Research (N-00014-89-J-1515).]

\section{0:55}

2aAO5. Dominant scattering mechandsms and associated scattering models for several zooplankton types. Timothy $\mathrm{K}$. Stanton (Dept. of Appl. Ocean Phys. and Eng., Woods Hole Oceanogr. Inst.. Woods Hole, MA 02543), Dezhang Chu, and Peter H. Wiebe (Woods Hole Oceanogr. Inst., Woods Hole, MA 02543)

The shape and material properties of zooplankton can vary widely from species to species and, as a result, the acoustic scattering properties vary accordingly. Because of the great complexity of the boundary conditions of the animals, determination of the conditions is difficult, if not impossible, by direct measurement. Through a series of controlled laboratory measurements of acoustic backscatter by live individual animals, the boundary conditions of several different types of animals have been inferred. Dominant scattering mechanisms of the animals have been identified and incorporated into approximate scattering models. Data and models are presented for live individual euphausiids (weakly scattering fluidlike), gastropods (hard elastic shelled), and siphonophores (gas-bearing). In particular, relative scattering efficiency (echo energy per unit biomass) and patterns of target strength versus frequency for both single echoes from individuals as well as averages from many echoes will be shown. [Work supported by ONR and NSF.]

\section{Contributed Paper}

11:20

2aAO6. Acoustic classification of zooplankton. Linda V. Martin (Dept. of Biology, Woods Hole Oceanogr. Inst., Woods Hole, MA 02543), Timothy K. Stanton, Peter H. Wiebe, and James F. Lynch (Woods Hole Oceanogr. Inst., Woods Hole, MA 02543)

Accurate acoustic characterization of zooplankton species is essential if reliable estimates of zooplankton biomass are to be made from acoustic backscatter measurements of the water column. Work on the forward problem has resulted in the identification of three categories of scatterers, represented by theoretical scattering models [Stanton et al., ICES J. Marine Sci. 51, 505-512 (1994)]: gas-bearing (e.g., siphonophores), fluidlike (e.g., euphausiids), and hard elastic-shelled (e.g., pteropods). If there are consistent differences in the characteristic acoustic signatures of each of these classes of zooplankton, it should be possible to solve the inverse problem by using acoustic backscatter data to mathematically infer the class of scatterer. Two different inversion techniques are applied to a dataset of several hundred pings collected from broadband insonifications $(\sim 350-750 \mathrm{kHz})$ of live zooplankton tethered and suspended in a large tank filled with filtered seawater. The model-based classifiers depend on comparison of the data with theoretical scattering models, whereas the feature-based classifiers are independent of the models and exploit only the inherent characteristics of the acoustic returns. Preliminary results indicate that the acoustic classification of zooplankton insonifications into categories representing distinct scatterer-types is feasible. 
Session 2aEA

\title{
Engineering Acoustics and Speech Communication: Recent Advances in Hearing Aid Technology
}

\author{
Sigfrid D. Soli, Cochair \\ House Ear Institute, 2100 West Third Avenue, Los Angeles, California 90057 \\ James M. Powers, Cochair \\ Naval Undersea Warfare Center, Code 2131, New London, Connecticut 06320
}

Chair's Introduction-8:00

Invited Papers

8:05

2aEA1. Where are we now? A 30-year perspective. Mead C. Killion (Etymotic Res., 61 Martin Lane, Elk Grove Village, IL 60007)

At one time, most of the things known about hearing aids were wrong. The resulting hearing aid designs often made it more difficult to hear in noise rather than less: In 1970, Tillman et al. reported a 20-dB degradation in the signal-to-noise ratio corresponding to a 50\% word-recognition score. The situation has improved because of improved components and especially because of an improved understanding of what a hearing aid can do to help [or make things worse, such as "filtering out the noise," which unavoidably results in the loss of valuable speech cues as well (Villchur, 1993)]. Most modern hearing aids can provide improved hearing in noise, and some of them can handle high-level noise and music without audible distortion. One design is being successfully used in concerts by members of major symphonies. Nonetheless, even the best—and best fitted-hearing aids typically leave their wearer with a deficit of at least 2-3 dB in tolerable signal-to-noise ratio (mild-moderate loss), often 6-8 dB (moderate loss), and sometimes much more. Objective improvements in signal-to-noise ratio appear needed to close the gap.

\section{8:30}

2aEA2. An overview of the completely-in-the-canal hearing aid. Jeremy Agnew (Starkey Labs., Inc., Colorado Res. Lab., P.O. Box 8100, Colorado Springs, CO 80933)

As the ability to miniaturize electronic circuitry and components has progressed, modern hearing aids have become smaller and smaller. This trend has resulted in the completely-in-the-canal (ClC) hearing aid, which is the smallest hearing aid available today. This paper will provide an overview of the background, technology, and acoustics of CIC hearing aids. Topics to be covered include the origins and evolution of the CIC, miniaturization of components, and allied advances in enabling technology. Emphasis will be placed on the acoustic advantages of placing the CIC hearing aid deep in the auditory canal, including the effects on gain and distortion, the resulting frequency response in the ear, the significance of localization, the use of natural pinna cues, and the reduction of the occlusion effect. These topics will also be related to normal binaural hearing. The results of objective and subjective testing in these areas will be included. The paper will conclude with examples of challenging patient fittings that are possible with CIC hearing aids.

\section{$8: 55$}

2aEA3. Directional hearing aid based on array technology. Wim Soede (ARDEA, Harmen Doumastraat 24, NL 2321 JL Leiden, The Netherlands), Mead C. Killion, and Matthew J. Roberts (Etymotic Res., 61 Martin Lane, Elk Grove Village, IL 60007)

Hearing-impaired listeners often have great difficulty understanding speech in surroundings with background noise or reverberation. Directional-microphone hearing aids can often reduce background noise in relation to the desired speech signal. To this end at the Delft University of Technology in The Netherlands, microphone systems were developed with strongly directional characteristics, using array techniques. Considerable attention was paid to optimization and stability. Free-field simulations of several robust models showed that a directivity index of $9 \mathrm{~dB}$ can be obtained. Simulations were verified with a laboratory model. The results of the measurements agreed well with the measurements, and based on these two, portable models were developed. The benefit of these microphone arrays for the hearing impaired was tested in a measurement setup with eight noise sources, simulating a true cocktail party. The results of the listening tests with 44 hearing-impaired subjects will be presented showing an average improvement of the $\mathrm{S} / \mathrm{N}$ ratio of $7.0 \mathrm{~dB}$ for monaural fitting. The first results of research on another array type will be discussed.

9:20

2aEA4. Improvements in hearing aid transducers. Earl R. Geddes (Knowles Electronics, 1151 Maplewood Dr., Itasca, IL 60143)

Hearing aid transducers have been around for nearly 50 years and have reached a significant level of maturity. Recent improvements have been made primarily in the areas of robustness, size, and integration of subsystems. This presentation will describe several recent improvements in hearing aid transducers. The author will also discuss areas where future improvements are likely to be made. A discussion of the noise levels in microphones, which are felt to be a concern of the industry, will be made in an attempt to present a manufacturer's view of this issue. 
2aEA5. A micromachined silicon condenser microphone and its potential for hearing aid applications.. Jonathan J. Bernstein (The Charles Stark Draper Lab., MS 37, 555 Technology Square, Cambridge, MA 02139)

Draper Laboratory has fabricated and tested several generations of micromachined silicon microphones. These monolithic microphones include on-chip JFET buffer amplifiers, providing a complete microphone plus electronics on a single silicon chip. Recent test data will be presented showing sensitivities as high as $40 \mathrm{mV} / \mathrm{Pa}(-48 \mathrm{~dB}$ re: $1 \mathrm{~V} / \mu \mathrm{Bar})$, with routine sensitivity of $15 \mathrm{mV} / \mathrm{Pa}$ $(-57 \mathrm{~dB} r e: 1 \mathrm{~V} / \mu \mathrm{Bar}$ ) and bandwidth of $70 \mathrm{~Hz}$ to $20 \mathrm{kHz}$. The particular constraints resulting from use as a hearing aid microphone are the available bias voltage (one volt at end of battery life) and the small package volume. An analysis of the theoretical attainable sensitivity of a micromachined microphone will be presented. This analysis shows that useful sensitivities ( $15 \mathrm{mV} / \mathrm{Pa}$ ) can be attained with a $\mathrm{I}-\mathrm{V}$ bias in a small $\left(15 \mathrm{~mm}^{3}\right)$ internal package volume. [Work supported by Noise Cancellation Technologies, Inc.]

\section{0:25}

2aEA6. Micromachined silicon microphones and hearing aids: Performance and potential. Victor Nedzelnitsky (Natl. Inst. of Standards and Technol., Sound Bldg. [233], Rm. A147, Gaithersburg, MD 20899-0001)

Small size, good frequency response characteristics, wide dynamic range, low cost, low sensitivity to external influences such as vibration, and low power requirements (extended battery life) of associated electronics have long been recognized as desirable properties of microphones used in hearing aids. New technologies of micromachined silicon capacitive microphones show promise not only for present hearing aid applications, but also for evolving array-based ones that may become practical for improving speech reception in the presence of noise and reverberation. Stability of microphone sensitivity can be particularly important in maintaining the designed directionality of arrays during their service lifetime. Designers of new microphones need reliably measured performance data to develop these new technologies and to optimize designs for specific applications. NIST measurement services long available to the public have been extended to obtain these data. Examples, including free-field sensitivity level versus frequency characteristics, are discussed for some experimental prototype omnidirectional microphones recently designed by $J$. Bernstein of Draper Laboratory, a customer of these services. Some possible lines of research and development of micromachined silicon transducers for hearing aid applications are suggested and discussed.

\section{Contributed Papers}

\section{0:50}

2aEA7. Temporal amplitude modulation processing for phonetic “edge enhancement" for otopathologic listeners. Margaret Mortz (Dept. of Eng., Univ. of Denver, Denver, CO 80208), Christopher Schweitzer, and Mark Terry (AudioLogic, Inc., Boulder, CO 80301)

This research attempts to address the problem of hearing in noise by hearing-impaired listeners with digital algorithms motivated by the results of Carrell and Opie, 1992, who showed that temporal amplitude modulations helped normal-hearing listeners form auditory objects when exposed to time-varying sinusoidal sentences. The current processing algorithms involved segmenting speech materials into spectral bands and extracting a signed envelope from each band. The envelope in each band was then normalized and either directly added back to the normalized signal or "edge enhanced" with a derivative operator before recombination with the normalized signal. A compression algorithm using a hyperbolic tangent was used to compress the enhanced speech before renormalizing to the same power (rms) as the original speech. Processing variations included applying coherence rules across bands to ascertain when it was "appropriate" to modulate the signal with this technique. Standardized (BKB) sentences were scored for seven hearing-impaired listeners with and without the modulation processing. As a further control, preemphasized trebleenhanced samples were also compared. The same materials were used for subjective judgments on scaled perceptual difference measures, again comparing processed versus the unprocessed speech samples. Both types of measures produced encouraging results.

\section{1:05}

2aEA8. Open audiometric reporting standard (OARS). David J. Delage (Qualitone, 4931 w. 35th St., Minneapolis, MN 55416), Brenda Dean (DBC-Mifco, Portsmouth, NH 03801), and Jerry L. Yanz (Qualitone, Minneapolis, MN 55416)

Communicating data and/or graphs from hearing test instruments to hearing-aid-fitting systems or office management systems which are typically produced by different manufacturers has proved an impediment to the use of programmable hearing aids. In an attempt to resolve this difficulty, a flexible standard software protocol for communicating data and graphs between PC-based systems has been developed by Qualitone and has been offered royalty-free to the hearing health community. Called the open audiometric reporting standard (OARS), it is based on the existing Windows open database connectivity (ODBC) standard. The purpose of OARS is to allow systems to store or transfer data or graphical information in an open and standardized way, independent of the database language. Effort has been made to insure compatibility with all Windows-based systems (including test instruments, hearing aid programmers, and office management systems) and to provide a method whereby all possible system configurations and their data and graphic needs can be supported.

\section{1:20}

2aEA9. Feedforward compression algorithm to compensate for the loudness recruitment in hearing-impaired people. Zlatan Ribic and Jun Yang (Viennatone GmbH, Frobelgasse 26-32, A-1164 Wien, Austria)

In the proposed compression algorithm, Hilbert transform and vector sum generator are used to obtain the Hilbert envelope AM which is the input signal of the following attack/release network (ARN). ARN is simulated by a first-order, low-pass filter with a variable time constant. Signal AM is also processed by a first-order high-pass filter (its time constant is about $10 \mathrm{~ms}$ ) to provide a signal $B$ for controlling the time constant of the ARN in such a way that the time constant is the attack time with the recommended range from 0.1 to $10 \mathrm{~ms}$ when $B$ is positive, and the release time with the recommended range from 10 to $100 \mathrm{~ms}$ when $B$ is negative. Finally, a voltage-controlled amplifier is used to amplify the original signal with the gain inversely proportional to the power function of the signal at the output of ARN. The desired compression ratio can be oblained by changing the power. Simulation results have shown that the proposed compression algorithm is simple and applicable for not only the wideband channel, but also multiband channels. In addition, the compression parameters can be adjusted according to the designer's requirement. 


\title{
Session 2aNS
}

\author{
Noise: Flow Induced Noise \\ Gerald C. Lauchle, Chair \\ Graduate Program in Acoustics, Applied Science Building, Pennsylvania State University, P.O. Box 30, \\ State College, Pennsylvania 16804
}

\author{
Chair's Introduction-8:30 \\ Invited Papers
}

$8: 35$

2aNS1. Recent developments in computational aeroacoustics. Lyle N. Long (Penn State Univ., 233 Hammond Bldg., University Park, PA 16802)

There is renewed interest in predicting aeroacoustic noise, especially for jets, rotating blades, and shock waves. These flows involve nonlinear, three-dimensional, turbulent phenomena, and nonuniform free streams. Simulating these flows requires algorithms quite different than those traditionally used in computational fluid dynamics (CFD). The time-dependent nature of aeroacoustic problems requires the algorithm to correctly simulate the dispersion and dissipation features of the flow. Good CFD algorithms usually rapidly damp out all but the steady-state portion of the flow, and are inappropriate for aeroacoustics. Computational aeroacoustics schemes have more in common with large eddy simulation (LES) algorithms than those used in CFD. Recent progress in higher-order algorithms for supersonic jets [T. S. Chyczewski and L. N. Long, 16th AIAA Aeroacoustics Conference, Paper 95-011 (1995)] and fan noise [Y. Ozyoruk and L. N. Long, 16th AIAA Aeroacoustics Conference, Paper 95-063 (1995)] illustrates that quite complicated aeroacoustic problems can be simulated. These algorithms require roughly 5-10 grid points per wavelength. The large demand on computer memory and speed requires that one use modern parallel computers, such as the IBM SP2 and the TMC CM-5. One must be careful to properly load balance the scheme and to minimize interprocessor communication. Kirchhoff surfaces are very effective in predicting the far-field solution.

\section{9:05}

2aNS2. An analytical and statistical examination of the low-wave-number region of the turbulent boundary layer wall pressure. Y. F. Hwang (Naval Surface Warfare Crr., Carderock Div., Code 7200, Bethesda, MD 20084)

The underlying physical mechanisms that generate the low-wave-number pressure components of the turbulent boundary layer wall pressure are examined based on a solution of linearized Navier-Stokes equations in the viscous sublayer with no-slip wall condition. The result indicates that the predominant low-wave-number pressure components are generated by viscous diffusion of shear stress fluctuations at the no-slip wall. These random pressure components have a wave-number spectrum spanning from zero wave number to well beyond the convective wave number. However, their contribution to the convective ridge of the wall pressure spectrum is negligible compared to those of other sources. In the low-wave-number limit, we reach the same conclusion reached by Chase [J. Fluid Mech. 225, 545-555 (1991)], i.e., the wave number spectral density does not vanish as the streamwise wave number approaches zero. The reasons for nonvanishing wave-number spectral density as $k \rightarrow 0$, for both shear stress and wall pressure, are established analytically. A statistical model is derived based on a probabilistic area-averaging on an assumed random process which is capable of producing the measured two-point correlation functions. The result is a space-time autocorrelation function that yields the experimental low-wave-number data. [Work supported by NSWC ILIR Program and ONR.]

\section{9:35}

2aNS3. Pressure mapping under an operating lawn mower deck. Jeffery A. Giordano (Deere \& Co. Technical Ctr., 3300 River Dr., Moline, IL 61265)

The use of relatively quiet engines and electric motors on lawn mowers has shifted much of the emphasis on noise reduction to the under deck flow-induced noise sources. However, the environment under an operating mower deck makes it difficult to either model or directly measure the pressure field. This presentation will detail a rather simple scheme which was used to first measure, then animate the pressure field in a plane directly below an operating deck, using an inertial frame of reference. Time synchronous averaging was employed to collect the data at discrete sample points. Individual data planes were then reconstructed representing all the sample points at specific blade locations. The measurement planes were contoured and used as separate frames in a slow-motion animation of the pressure field. 
2aNS4. Active control of axial-flow fan noise. John R. MacGillivray, Gerald C. Lauchle, and David C. Swanson (Graduate Prog. in Acoust. and Appl. Res. Lab., Penn State Univ., P.O. Box 30, State College, PA I6804)

An investigation was conducted using active noise control to reduce noise from small axial-flow fan units commonly found in computers and printers. The fan unit itself was used as the cancellation source in the active noise control scheme, achieved by axially modulating the unit with a shaker. Feasibility studies which looked at radiation efficiency and transfer function data identified fan units which would adequately perform as efficient, undistorted sources of noise when driven by particular shakers. Once a suitable shaker and fan combination was discovered, simulations of active noise control were conducted in MATLAB which utilized the measured error path impulse response (representing the system which defines the output voltage response of a microphone near the fan to an input voltage supplied to the shaker). Results from the simulation showed that an experiment could be constructed which would effectively reduce the tonal components from the fan unit. An experimental demonstration was constructed, results from which show a 20- $\mathrm{dB}$ reduction in sound-pressure level for the blade passage tone, a 15-dB reduction for the second harmonic, and a 7- to 8- $\mathrm{dB}$ reduction for the third harmonic. [Work supported by IBM through Shared University Research Program.]

$$
\text { 10:35-10:50 Break }
$$

\section{Contributed Papers}

10:50

2aNS5. Effects of installation for computer cooling fans. Jacklyn D. Bezemek and Luc Mongeau (Dept. of Mech. Eng., Purdue Univ., 1077 Herrick Lab., W. Lafayette, IN 47907)

The aerodynamic performance and the noise emissions of computer cooling fans are influenced by the design of the computer enclosure and its internal components. Moreover, the coupling between the spatially distributed source and the acoustic response of the system hampers the use of most linear electroacoustic source filter models. Employing a method derived from acoustic similarity laws [Neise and Barsikow, "Acoustic Similarity Laws for Fans," Trans. ASME 104 (May 1982)], the installation effects of three small axial cooling fans mounted in various computerpower supply assemblies have been determined by combining measurements in siru and standardized fan sound power measurements. It was found that flow performance may be reduced, additional sound may be generated due to inflow inhomogeneities, and the fan itself may affect the fundamental Helmholtz resonance of the computer and power supply cavilies.

\section{1:05}

2aNS6. Numerical simulation of aerosound from airfoils and exhaust jets using Lighthill theory with $k-\epsilon$ turbulence model and large eddy simulation. Wei-Jen Huang, David B. Schein, and William C. Meecham (Dept. of Mech. and Aerospace Eng., Univ. of California, Los Angeles. CA 90095)

Far-field noise from turbulence near an NACA 0012 airfoil and potential core of a high-speed (subsonic) jet engine exhaust have been computed using the ANSWER computational fluid dynamics code of Runchal and Caretto [Intl. J. Num. Methods Eng. 24 (1990)] and Lighthill's acoustic analogy. The $k-\epsilon$ turbulence model which is part of ANSWER is used to compute Reynolds stress from two "side" equations in addition to continuity, Navier-Stokes, and for high-speed flow, the energy equation. Threedimensional turbulence intensities are obtained from the stress. Fluctuations are needed to calculate aerosound, while $k-\epsilon$ only produces averages. Thus the time derivative form of Lighthill's analogy is used to calculate the sound. Time derivatives must be estinated; the characteristic time $L / u^{\prime}$, as calculated by $k-\epsilon$, is used. ANSWER modified to perform Large Eddy Simulation is used for the free jet, employing a hybrid subgrid scale model previously developed for application to a low-speed jet by Lee [UCLA dissertation (1992)] which combines the conventional Smagorinsky formulation with a deductive model formed by taking a Taylor series expan. sion of the flow field and using the first terms to give the fluctuations. Preliminary results are compared with jet noise measurements.

\section{1:20}

2aNS7. Application of describing-function theory to a flow-excited resonator. Hyungseok Kook and Luc Mongeau (School of Mech. Eng., Purdue Univ., 1077 Herrick Lab., W. Lafayetre, IN 47907)

A theoretical model based on a describing-function theory [T. D. Mast and A. D. Pierce, J. Acoust. Soc. Am. 97, 163-172 (1995)] was applied to the problem of a flow-excited Helmholtz resonator with a complex geometry. The lumped acoustic parameters and flow parameters required in the model were determined both from empirical data and from existing analytical models. Detailed comparisons were made between experiments and theory in order to validate the approach. The results show that the theory provides an alternative to computationally intensive CFD models for predicting the main acoustic characteristics of the resonator system.

\section{1:35}

2aNS8. The intake and exhaust noise control by influence on manifolds of vehicle engine. Roudolf Starobinski and Jean Kergomard (Laboratoire d'Acoustique, URA 1101 CNRS, Ave. Olivier Messiaen, BP 535, 72017 Le Mans Cedex, France)

Excitation of eigenmodes in intake and exhaust manifolds of multicylinder internal combustion engine is investigated. It is shown that some of the eigenmodes do not depend very much on the intake, and exhaust systems are jointing behind the manifold. The sound increase (particularly for an asymmetrical excitation) caused by these modes depends on the degree of the asymmetry of the manifold and on sound energy dissipation in the manifold. Under some conditions this increase is the reason for resonance sound radiation. The tendency for resonance excitation at the branch of intake (exhaust) pipes with mufflers is considered to be one of the criteria in assessing the quality of manifold construction. Special attention is paid to the asymmetrical exhaust and intake manifolds of trucks. It is shown that the influence of the manifolds design of their engines allows a decrease truck intake and exhaust noise in a wide range of crankshaft RPM. 


\title{
Session 2aPA
}

\section{Physical Acoustics: Nonlinear Acoustics of Rocks I}

\author{
Andrew N. Norris, Chair \\ Department of Mechanics and Aerospace Engineering, Rutgers University, P.O. Box 909, Piscataway, \\ New Jersey 08855-0909
}

Chair's Introduction-9:00

Invited Papers

\begin{abstract}
9:05
2aPA1. Measurements of third-order elastic constants in rocks. Kenneth W. Winkler (Schlumberger Res., Old Quarry Rd., Ridgefield, CT 06877) and Xingzhou Liu (Western Atlas Logging Services, Houston, TX 77042)

Third-order elasticity successfully describes a variety of acoustic phenomenon in many materials, and measurements exist of third-order elastic constants in metals, plastics, and crystals. A key feature of third-order elasticity theory is that acoustic velocities vary with the stress in a material. In spite of the fact that velocities in rocks are extremely stress dependent, no measurements have previously been published of third-order elastic constants in sedimentary rocks. This experimental technique is based on measuring changes in velocity with applied stress, both uniaxial stress and hydrostatic stress. By measuring five acoustic modes there is an overdetermined system of five equations and three unknowns. Measurements are reported of the three third-order elastic constants for nine rocks (sandstones, limestones, granite) and a few synthetic materials at ambient room conditions. Along with velocity, porosity, density, and uniaxial strength, these data constitute a unique database of rock properties.
\end{abstract}

2aPA2. High amplitude, bifrequency experiments in porous rocks. Thomas J. Plona, Bikash Sinha, Ralph D'Angelo, Chris Kimball (Schlumberger-Doll Res., Ridgefield, CT 06776), B. J. Landsberger, and Mark F. Hamilton ( Univ. of Texas, Austin, TX 78713)

Fluid-filled, porous, sedimentary rocks have the general characteristic that the sound speeds are strongly dependent on the state of stress and therefore, the third-order elastic coefficients (i.e., $A, B, C$ ) are generally much larger than for normal solids. However, the linear attenuation can also be very large such that in nonlinear wave propagation, the Gol'dberg number is small. Experiments studying harmonic generation in porous rocks have been made using an ultrasonic immersion system (i.e., water/solid/water) where a high amplitude (e.g., $500 \mathrm{kPa})$, bifrequency $(0.95$ and $1.05 \mathrm{MHz}$ ), tone burst is emitted and then sum and difference frequencies are detected after propagation through the water/solid/water system. A frequency domain numerical implementation of the KZK (KhokhlovZabolotskaya-Kuznetsov) nonlinear parabolic wave equation is used to describe the nonlinear propagation in the three layer system. Experiments on solids with well-known acoustic properties (linear and nonlinear) were used to validate the model. Measurements were then made on several sandstones and limestones and the model used to derive the nonlinear propagation parameter, $\beta=f(A, B, C)$. Finally, these results are compared with independent measurements of $A, B, C$ for these rocks.

\section{9:50}

2aPA3. Estimation of stress-induced velocity anisotropy using finite-deformation theory. Xingzhou Liu (Westem Atlas Logging Services, 10201 Westheimer, Houston, TX 77042) and Amos Nur (Stanford Univ., Stanford, CA 94305)

Mumaghan's second-order elasticity theory is applied to studying the directional- and stress-dependence of elastic wave velocities in intrinsically isotropic rocks. At any stress within the "perfectly" elastic range, the velocity change with stress (slope) and direction is determined by three third-order elastic (TOE) constants. However, the three TOE constants are not all independent for most rock samples measured in this study. This observation reduces the number of independent TOE constants from three to two, which allows the prediction of stress-induced velocity anisotropy from $P$ - and $S$-wave velocity measurements under hydrostatic pressures. Compared with the approach that adopts linear stress-strain relation but allows stress-dependent elastic constants (second-order), this method avoids the modeling of rock pore compressibilities and has a clearer physical meaning. The origin of the elastic nonlinearity in rocks is shown to be the prevalence of compliant pore space in rocks, with geometries ranging from thin cracks in igneous rocks to cemented grain contacts in granular sedimentary rocks undergoing diagenesis. By using elastic solutions for cracks, contacts, and cemented contacts and assuming a mixture of these different types of pores, it is shown that the TOE constants of rocks can be related to their textural or petrographical properties. 
2aPA4. Nonlinear acoustics of rocks and other hard subjects. M. A. Breazeale (Natl. Ctr. for Physical Acoustics, University of Mississippi, University, MS 38677)

With single crystals, an acceptable approximation is achieved with only the first nonlinear term in the wave equation. With such crystals and weakly nonlinear solids, one can define a nonlinearity parameter $\beta$ as the negative ratio of the coefficients of the nonlinear and the linear terms. Values of $\beta$ between 3 and 15 have been observed for single crystals. It appears that the approximation no longer is adequate when $\beta$ becomes larger. Values of $\beta$ berween 80 and 1000 have been observed for rocks. A value of $\beta=1500$ has been observed for PZT at the Curie temperature. In addition, frequency dispersion of the nonlinearity has been observed in PZT, and the third harmonic is much larger than expected from an extrapolation of second harmonic data. Since the approximate theory no longer is adequate, terms have been added to the nonlinear equation. The nonlinear equation required to fit data on PZT has been determined. The next step is to explain this mathematical success in physical terms. This will involve the effect of grain boundaries. Then the results can be applied to rocks, which are more complicated.

\section{0:35-10:50 Break}

\section{0:50}

2aPA5. Nonlinear vibroacoustic properties of rocks. Lev A. Ostrovsky (Univ. of Colorado, CIRES/NOAA Environ. Technol. Lab., 325 Broadway, Boulder, CO 80303)

This is a summary of recent theoretical and experimental work performed by the Nonlinear Acoustic Group (L. Ostrovsky, A. Sutin, V. Nazarov, I. Belyaeva, Yu. Zaitsev) at the Institute of Applied Physics, N. Novgorod, Russia. Experiments show that Earth materials can have anomalously strong mechanical nonlinearity. However, in many cases there is a lack of clear understanding of specific mechanisms responsible for these nonlinear effects. Some theoretical models of structurally inhomogeneous media have been constructed and experimentally verified. These models include: (1) porous waterlike media characterized by small (but finite) shear modulus; (2) grainy media where nonlinearity is caused by the intergrain contacts (Hertz model); (3) some nonlinear models of cracks. Laboratory experiments have demonstrated a good agreement with theoretical results, and in all three cases the values of the nonlinearity parameter (defined similarly to that used in nonlinear acoustics for gases and liquids) could achieve the values of the order of $10^{3}-10^{4}$ and even more. Some estimates for real rocks are also given. They show that nonlinear parameters may prove to be much more sensitive to the details of the material structure than the usual linear ones (e.g., sound velocity). This can serve as a base for developing new methods of seismic surveillance.

\section{Contributed Papers}

\section{1:15}

2aPA6. Borehole propagation in berea sandstone: Stress-induced dipole anisotropy. Bikash Sinha, Thomas J. Plona, Kenneth Winkler, and Ralph D'Angelo (Schlumberger-Doll Res., Ridgefield, CT 06776)

Stress-induced dipole anisotropy exhibits a crossover in the principal flexural wave slowness dispersions oriented parallel and perpendicular to the farfield uniaxial compressive stress direction. This crossover phenomena is a result of borehole stress concentrations and can be used as a new technique for distinguishing stress-induced anisotropy from intrinsic anisotropy. Theoretical modeling (based on third-order elasticity) and laboratory measurements (from 10 to $60 \mathrm{kHz}$ ) have been made on a large block of berea sandstone subject to a uniaxial stress of up to $5 \mathrm{MPa}$. The two flexural mode dispersions are obtained by Prony's processing of an array of measured waveforms for dipole orientations parallel and perpendicular to the stress direction. The theoretical dispersions in the presence of biasing stresses are obtained from the solution of equations of motion for small dynamic fields superimposed on a static bias. Good agreement has been obtained between theory and experiment including a dispersion crossover phenomena unique to stress-induced anisotropy-

\section{1:30}

2aPA7. Variational approaches in the nonlinear theory of fluid-permeable elastic continua. Michael G. Grinfeld and Andrew N. Norris (Dept. of Mech. and Aerospace Eng., Rutgers Univ., Piscataway, NJ 08855-0909)

A new nonlinear goveming system for poroelastic fluid-permeable media in Eulerian variables is derived on the basis of Hamilton's principle for reversible effects and the Onsager-Sedov approach for irreversible effects. The classical (Murnaghan-like) equations of nonlinear elasticity, as well as the governing equations of the ideal and Navier-Stokes fluids, appear to be some special cases of the general governing system. It is well known that the Navier-Stokes equations for compressible fluids provide a correct self-consistent basis for studying a wide variety of nonlinear effects in fluids. The authors believe that the governing system proposed here provides the same opportunities for various nonlinear effects in poroelastic fluid-penetrable media. In particular, it allows one to study the internal structure of shock waves and flutterlike phenomena among others. [Work was supported by ONR.]

\section{1:45}

2aPA8. Elastic nonlinearity in rock: On the relative importance between higher-order elastic constants and hysteresis. Koen Van Den Abeele. Paul Johnson, and James Ten Cate (EES-4 MS D443, Los Alamos Natl. Lab., Los Alamos, NM 87545)

Rocks are extremely elastically nonlinear, even at strain as low as $10^{-7}$. Recent simulations of dynamic elastic pulsed wave experiments and comparison with static and resonance test predictions revealed that the physical mechanism for nonlinearity in rocks cannot be attributed to higher-order nonlinear coefficients alone. Static stress-strain tests and resonance measurements show in addition an undeniable hysteretic behavior of stress and modulus versus strain. Therefore, hysteresis has been introduced into the dynamic nonlinear wave equation by means of a discontinuous term in the modulus. The new theoretical model is based on four parameters: the first and second nonlinearity constants, attenuation, and hysteresis strength. In doing so, rich hannonic spectra and nonlinear waveforms observed in dynamic pulse mode experiments can be simulated using realistic values of higher-order elastic constants and hysteresis. Furthermore, the model provides characterization criteria for rock types depending on the relative importance of hysteresic and nonlinearity parameters. Chalk, for instance, can have large first and second nonlinearity parameters, because it shows a rich harmonic spectrum but no hysteresis. On the other hand, the nonlinear spectra of several sandstones can be attributed almost entirely to the first nonlinear coefficient and to hysteresis. [Work supported by DOE/OBES/UCal.] 
Session 2aSA

\title{
Strucutral Acoustics and Vibration: Plates and Beams
}

\author{
Joseph M. Cuschieri, Chair \\ Department of Ocean Engineering, Center for Acoustics and Vibration, Florida Atlantic University, \\ Boca Raton, Florida 33431
}

Contributed Papers

9:00

2aSA1. Application of Skudrzyk's “mean-value theory" to fluid-loaded plates. Rendell R. Torres, Victor W. Sparrow, and Alan D. Stuart (Grad. Prog. in Acoust., Penn State Univ., P.O. Box 30, State College, PA 16804)

The geometric-mean drive-point admittance of a complex structure can be found by examining the admittance of the corresponding infinite structure (i.e., "characteristic admittance," $Y_{c}$ ] [Skudrzyk, J. Acoust. Soc. Am. 67, 1105-1135 (1980)]. The frequency response of an infinite plate, for example, coincides with the geometric-mean response of a finite plate, i.e., the response equidistant from the resonance maxima and antiresonance minima, plotted on a logarithmic scale. Skudrzyk's "mean-value theorem" was derived (and experimentally verified) without consideration of fluid coupling, which introduces a reactive effect that physically resembles a mass loading. The purpose of this research is to find whether the response of the fluid-loaded infinite plate still corresponds to the geometric-mean response of the fluid-loaded finite plate. Numerical results indicate that, in the presence of fluid loading and at low frequencies (below critical), the mean-line drive-point admittance of the finite plate still corresponds to the infinite-plate drive-point admittance that has been derived analytically [Crighton, J. Sound Vib. 54, 389-391 (1977)]. [Work supported by the Applied Research Laboratory.]

9:15

2aSA2. An acoustic boundary element method using analytical/numerical matching applied to a three-dimensional fluid-loaded plate. Ronald J. Epstein and Donald B. Bliss (Dept. of Mech. Eng. and Mater. Sci., Duke Univ., Box 90300, Durham, NC 27708-90300)

Analytical/numerical matching (ANM) is a hybrid scheme combining a low-resolution global numerical solution with a high resolution local analytical solution to form a composite solution. ANM is applied to a harmonically vibrating flat plate in three dimensions to calculate the radiated acoustic field and the associated fluid loading. The problem utilizes overlapping smoothed dipoles, and local corrections to calculate the dipole strength distribution on the surface of the plate. A smoothing length scale is introduced that is larger than the smallest physical scale, and smaller than the largest physical scale. The global low-resolution solution is calculated numerically using smoothed dipoles, and converges quickly. Local corrections are done with high-resolution local analytical solutions. The global numerical solution is asymptotically matched to the local analytical solutions via a matching solution. The matching solution cancels the global solution in the nearfield, and cancels the local solution in the farfield. The method is very robust, offering an insensitivity to collocation point position. ANM provides high-resolution calculations from low-resolution nu- merics with analytical corrections, while avoiding the subtlety involving singular integral equations, and their numerical implementation. [Work supported by ONR.]

9:30

2aSA3. Calculation of the total and individual powers of vibrating finite panel by mutual radiation impedance. Daiji Mikami, Akio Hasegawa, and Toshiaki Kikuchi (Dept. of Appl. Phys., Natl. Defense Acad. of Japan, 1-10 Hashirimizu, Yokosuka 239, Japan)

Recently the active acoustic control technique using the controllable secondary source has attracted much attention as an effective method to reduce the power output from the primary source. In this technique, essential reduction in the sound power output from the primary source is achieved by the acoustic destructive interaction effect which is characterized by mutual radiation impedance. In this paper, the mutual radiation resistance of two identical rectangular panel cells $(a \times b)$ simply supported in an infinite baffle is determined from the total power output radiated to the farfield. Numerical values of the mutual radiation resistance are given as a function of $k a$ for rectangular panel cells with various aspect ratios and orientation angles. The 3-D representations of individual output power from a multiplicity of panel cells are shown for (a) even-even mode (quadrupole type), (b) even-odd mode (dipole type), and (c) odd-odd mode (monopole type). In some 3-D plots of odd-odd modes, for frequencies well below the critical frequency, the magnitude of the power output from each panel cell is observed to follow the velocity distribution of panel cells exactly.

\section{$9: 45$}

2aSA4. Radiated power and radiation efficiency of a point driven panel. J. Ertel (U.S. Naval Acad., Annapolis, MD 21402), G. Maidanik, and J. Dickey (David Taylor Res. Ctr., Bethesda, MD 20084)

The partial radiation efficiency from a line-driven panel was previously defined and investigated by the authors ["Partial radiation efficiency of line-driven panels," J. Sound Vib. 144, 71-86 (1991)]. In the present paper, the counterpart to the previous work is presented. It is shown that the radiation efficiency, like the partial radiation efficiency, is dependent on the mechanical damping in the panel; and again, the efficiency increases with increase in the mechanical loss factor in the panel. Also, the dependence of the radiation efficiency on fluid loading is analogous to that of the partial radiation efficiency described in the previous work. In this paper, in addition, the radiated power as a function of frequency is investigated. It is shown that the radiated power appropriately diminishes with an increase in the mechanical damping. This result, in contrast, shows the fallibility of the conclusion that "a higher radiation efficiency implies, without further qualifications, more radiated power." The results of computer experiments are cited in support of the various aspects in the paper. 
2aSA5. Acoustic scattering from a fluid-loaded elastic plate with a distributed inhomogeneity of varying length scales. Joe M. Cuschieri (Ctr. for Acoust. and Vib., Dept. of Ocean Eng., Florida Atlantic Univ., Boca Raton, FL 33431) and David Feit (David Taylor Res. Ctr., Bethesda, MD 20084)

Solutions were presented at the $128 \mathrm{th}$ and the 129 th ASA meetings for the response and acoustic scattering from a fluid-loaded elastic plate with a distributed mass or stiffness inhomogeneity. The distributions considered for the inhomogeneity were a uniform distribution, a quadratic distribution, and a biquadratic distribution. It was shown in the previous presentations that below the critical frequency, there is a marked difference in the scattering between a mass inhomogeneity and a stiffness inhomogeneity. Furthermore, the scattering from the stiffness inhomogeneities was much less than that from the mass inhomogeneities. Above the critical frequency. both types of inhomogeneities generated similar scattering results. In this paper the influence of variations within the distribution of the inhomogeneity is considered. That is, apart from the overall distribution, the inhomogeneity has local changes. The inhomogeneity has two length scales, one associated with the overall distribution, where the same types of distributions as previously are considered, and one associated with the changes within the inhomogeneity distribution. The scattering from stiffness-type inhomogeneities increases with the introduction of this second length scale, while the scattering from the mass inhomogeneities is less influenced by the introduction of the second length scale. These results agree with those presented by Steinberg and McCoy at the 129th ASA meeting. [Work sponsored by ONR.]

\section{0:15}

2aSA6. Computation of complex wave numbers and amplitudes on vibrating structures from response data. J. Gregory McDaniel, Kevin D. LePage, and Nathan C. Martin (Bolt Beranek and Newman Inc., 70 Fawcett St., Cambridge, MA 02138)

This work demonstrates a robust approach for computing complex wave numbers and amplitudes of waves in structures from experimental or numerical data. The approach postulates a wave field, which is a linear combination of damped waves. The number of waves and initial estimates of the complex wave numbers are based on any a priori physical knowledge and on the results of standard analyses of the data, such as wavenumber transforms and spatial attenuation rates. Given these initial estimates of wave numbers, associated wave amplitudes are computed by linear least-squares inversion to data. Optimization algorithms improve these estimates by searching for complex wave numbers and amplitudes that minimize the normalized mean-square error between the data and the wave field. This approach is often more robust than Prony-based techniques, which require equally spaced data and are more sensitive to noise or unmodeled components. The approach is demonstrated on experimental vibration measurements of a damped box beam. Loss factors are computed for traditional flexural waves as well as plate waves, which involve flexural motions of the walls of the box beam. [Work supported by ONR.]

\section{0:30-10:45 Break}

\section{0:45}

2aSA7. Experimental measurements of vibrational energy and acoustic radiation for a 3-D truss. Joseph E. Bondaryk, Ira Dyer, Leo Chiasson, and J. Robert Fricke (Dept. of Ocean Eng. MIT, 77 Massachusetts Ave., Rm. 5-435, Cambridge, MA 02139)

The MIT Structural Acoustics Group is making experimental measurements to determine the structural loss and radiation efficiency of an undamped, three-dimensional truss structure, constructed of 0.5 -in. aluminum tubing connected by massive aluminum joints. The truss was excited with a shaker driven by wideband white noise out to $20 \mathrm{kHz}$. The total vibrational energy in the truss was calculated by spatially integrating the energy estimated from acceleration measurements made over the entire truss. This value and the total input power, as measured with an impedance head, allow estimation of the total loss factor for the truss as a function of frequency. Total radiated sound power was calculated by spatially integrating a set of average acoustic intensity measurements made over a grid surrounding the truss. Since the total radiated sound power is known, the total loss factor can easily be broken down into a structural loss factor and a radiation loss factor. These measurements show that the radiation loss is smaller than structural loss, but larger than radiation from an equivalent length single strut would predict. The additional radiation is accounted for by a factor due to multipath in the truss. This factor is frequency dependent and can be estimated from the data by comparing to theory. [Research supported by ARPA and ONR.]

\section{1:00}

2aSA8. Acoustic radiation from a 3-D truss: Direct global stiffness matrix modeling results. J. Robert Fricke, a) Leo Chiasson, and Joseph E. Bondaryk (MIT, Rm. 5-218, 77 Massachusetts Ave., Cambridge, MA 02139)

This study was initiated to evaluate the nornalized sound power level radiated by a driven truss. The coincidence frequency for the beam elements was sufficiently high that global truss modes are not a concern. Radiation from local beam modes dominate the field. Acoustic radiation was modeled using a combination of the direct global stiffness matrix (DGSM) method and models based on radiation efficiency of cylindrical beams. Use of a nominal structural loss factor of $10^{-4}$ for the beam material (aluminum) overestimates the measured field by an order of magnitude. Earlier studies suggest that a combination of structural loss in the joints and multipath wave-type conversion in the truss leads to a loss factor of order $10^{-3}$. For this study experimental data were inverted to estimate a frequency-dependent loss factor, which confirms the prior estimate of order $10^{-3}$. When the DGSM based model was run with the estimated frequency-dependent loss factor, the model results match the measured data closely. A power balance shows that the structural loss factor dominates the total power dissipated in the system, as one might expect for a lightly fluid-loaded structure. [Research sponsored by ARPA/ONR.] a)E-mail: fricke@mit.edu

\section{$11: 15$}

2aSA9. Low-density granular fill for damping structural vibrations. J. Robert Fricke ${ }^{a)}$ and Mark A. Hayner (MTT, Rm. 5-218, 77 Massachusetts Ave., Cambridge, MA 02139)

Granular materials have been used for many years to damp structural vibrations. Often these treatments incorporate sand or lead shot. Both are heavy and provide some of their damping effect through mass loading. This paper discusses the damping properties of a low-density granular material, 3M glass microbubbles (tradename Scothlite). A paste was made using water and Scothlite and placed in an aluminum free-free beam. Resonant peaks of the beam were reduced by $10 \mathrm{~dB}$, and in some cases more. The specific gravity of the Scothlite is about 0.1 , so mass loading effects cannot account for the damping. Further, glass is not normally considered to be highly viscoelastic at room temperature. Rather, the attenuation mechanism is thought to be activated by the low bulk sound speed of the granular fill. With a low sound speed, the wavelength is short, and incipient attenuation in the fill becomes important. The mechanism is a combination of four possibilities: (1) small but finite intrinsic material attenuation, (2) frictional losses between rubbing grains, (3) nonlinear hys- 
teresis effects due to the Hertzian contact and deformation relaxation, and (4) viscous losses of the fluid flow between grains. [Research sponsored by ARPA/ONR.] ${ }^{\text {a) }}$ E-mail: fricke@mit.edu

\section{1:30}

2aSA10. Energy transport in a vibrating, extruding/intruding cantilever beam. Charles J. Konzelman and Marinos C. Stylianou (Dept. of Mech. Eng., Univ. of Victoria, Victoria, BC V8W 3P6, Canada)

Everyone has performed this experiment at one time or another: Take a ruler, hold it over the edge of a tabletop like a cantilever beam, pluck the end, and slowly retract the ruler into the tabletop. The frequency of the resulting vibration is observed to increase as the ruler is retracted into the tabletop. Although numerical simulations based on the linearized equation of motion for this intruding cantilever beam reveal that its total energy increases with the passage of time, the source of the energy is apparently unaccounted for. This study examines the transport of energy into (and out of) a cantilever beam which intrudes or extrudes from a rigid support with uniform axial velocity. By examining some second-order effects at the support and over the length of the beam, an attempt is made to define the mechanisms of energy transport into (and out of) the beam. This has potential application to the solution of transverse vibralion control problems, if the velocity of axial intrusion/extrusion is a properly prescribed function of time.

\section{1:45}

2aSA11. Applications of fractional calculus to the analysis of damped vibrations of viscoelastic oscillators. Yuriy A. Rossikhin (Dept. of Theoretical Mechanics, Voronezh State Acad. of Construction and Architecture, ul.Kirova 3-75, Voronezh 394018, Russia) and Marina V. Shitikova (Voronezh State Acad. of Construction and Architecture, Voronezh 394018, Russia)

Free damped vibrations of an oscillator, whose viscoelastic properties are described in terms of the fractional calculus Kelvin-Voight, Maxwell, standard linear solid models, and the models with a fractional operator, are determined. The problem is solved by the Laplace transform method. When passing from image to preimage, one is led to find the roots of an algebraic equation with fractional exponents. The method for solving such equations is proposed, which allows one to investigate the roots' behavior in a wide range of single-mass system parameters. A comparison between the results obtained on the basis of the four models has been carried out. It has been shown that for all models the characteristic equations do not possess real roots, but have one pair of complex conjugates, i.e., the test single-mass systems subjected to the impulse excitation do not pass into an aperiodic regime in any of the magnitudes of the relaxation and creep times. Once more, a model with some fractional operator is constructed, which allows the oscillator to be both in the vibrating motion and in the aperiodic regime depending on the intervals over which the relaxation times for the given model vary, as well as on the order of fractional power and the ratio of relaxed to nonrelaxed modulus.

\title{
Session 2aSC
}

\section{Speech Communication: Studies of Consonants, Non-Native Contrasts, Prosody, Stress and Rhythm (Poster Session)}

\author{
Keith R. Kluender, Chair \\ Department of Psychology, University of Wisconsin, 1202 West Johnson Street, Madison, Wisconsin 53706
}

\section{Contributed Papers}

\begin{abstract}
All posters will be on display from 9:00 a.m. to 12:00 noon. To allow contributors an opportunily to see other posters, contributors of odd-numbered papers will be at their posters from 9:00 a.m. to 10:30 a.m. and contributors of even-numbered papers will be at their posters from 10:30 a.m. to 12:00 noon. To allow for extended viewing time, posters will remain on display until 7:30 a.m. Wednesday morning.
\end{abstract}

2aSC1. Perceiving oral release rate of voiced stop consonants. David R. Williams (Sensimetrics Corp., 26 Landsdowne St., Cambridge, MA 02139)

Because of the differences in articulator mass and contact length, initial rales of increase in a constriction area at a consonant release may differ for stops produced at the various places of articulation. This paper examines the listener preferences for variations in the oral release rate (ORR) of syllable-initial labial, alveolar, and velar voiced stops. For each place of articulation, a five-member ORR continuum was synthesized using a set of articulatory parameters [K. N. Stevens and C. A. Bickley, J. Phon. 19, 161-174 (1991)] to control the rate of increase in the oral cross-sectional area at the stop release. ORRs were $15,20,30,50$, and $100 \mathrm{~cm}^{2} / \mathrm{s}$ for [b] and [d], and 10,15, 20,30, and $50 \mathrm{~cm}^{2} / \mathrm{s}$ for [g]. The synthesized $\mathrm{CV}$ syllables were presented in pairs to naive and experienced listeners for preference judgments. In general, faster ORRs were preferred for labial stops than for alveolar and velar stops. For the most stimuli, experienced listeners showed clearer preferences than did naive listeners. Normalized scores indicated that the range of preferred ORRs for alveolars was nar- rower than for labials and velars. Implications of these results for studies of phonetic prototypes will be discussed. [Work supported by NIH.]

2aSC2. Burst spectral measures and formant frequencies can be used to accurately discriminate place of articulation. Mark Hasegawa-Johnson (Res. Lab. of Electronics, MIT, 36-549. Cambridge, MA 02139)

Given an accurate burst location, four automatic measurements of the burst spectral shape and three semiautomatic formant onset measurements were used to correctly classify place of articulation of $90 \%$ of a corpus of 124 stops. At least 20 releases of each of the six English stops, in unconstrained prevocalic, preglide, or (for velar and labial stops only) pre-/r/ context, were chosen at random from the TIMIT corpus. Burst locations were marked by hand. The values of $F 2$ and $F 320 \mathrm{~ms}$ later, and of $\Delta F 2$ between 20 and $50 \mathrm{~ms}$ later, were measured automatically, and $18 \%$ of the tokens were corrected by hand. Burst compactness, peak frequency, and mid- and high-frequency amplitude were measured automatically. In separate analyses of variance, all seven measures showed significant place effects $(p<0.001)$. In three pairwise linear discriminants, the burst mea- 
sures alone were able to classify place with $85 \%$ accuracy, showing that automatic measurements can reproduce the result of Blumstein and Stevens [J. Acoust. Soc. Am. 64 (1978)]. With seven measures, the error rate was only $10 \%$. Most errors could be attributed to a strong labial burst and rapid formant transitions in $/ \mathrm{i} / \mathrm{or} / \mathrm{r} / \mathrm{context}$, or coloration of an alveolar burst by back-cavity coupling. [Supported by a grant from NIDCD.]

2aSC3. Locus equations as phonetic descriptors of consonantal place of articulation. Harvey M. Sussman and Jadine Shore (Dept. of Communicative Disorders \& Sciences, Univ. of Texas, Austin, TX 78712)

This experiment tested whether locus equation coefficients, slope, and $y$ intercept could serve as indices of place of articulation for obstruents sharing the same place of articulation across different manner classes. A previous study [C. A. Fowler, Percept. Psychophys. 55, 597-610 (1994)] reported that locus equations provided poor information for place because the mean slope for $/ \mathrm{d} /(0.47)$ was found to be statistically different than the mean slope for $/ z /(0.42)$ across ten speakers. Locus equations from 22 speakers were derived from $C V / U$ words with initial voiced oral stop $/ d$, voiceless aspirated stop $/ t^{\mathrm{h}} /$, nasal stop $/ \mathrm{n} /$, voice fricative $/ \mathrm{z} /$, and voiceless fricative $/ \mathrm{s} /$ preceding ten vowel contexts. Post-hoc tests revealed $/ \mathrm{d} /=/ \mathrm{z}=/ \mathrm{n} /$ for slope means. Slopes for $/ \mathrm{d} /$ and $/ \mathrm{t}^{\mathrm{h}} /$ were also equivalent when $F 2$ onset measurement points were corrected to account for aspiration. Scatterplots of slopes $x y$ intercepts revealed three nonoverlapping and distinct clusters of coordinates when the coronal group (/d, t, n, z, s/) was plotted versus labials and velars. A discriminant analysis using slope and $y$ intercept successfully categorized all coronals as alveolars with $87.1 \%$ accuracy. The collective results support the contention that locus equations can serve as effective descriptors of consonant place of articulation across manner classes. [Work supported by NIH.]

2aSC4. Categorization of stop consonants in $/ \mathrm{sCs} /$ context. Michael Kiefte and Terrance M. Neary (Dept. of Ling., Univ. of Alberta, Edmonton, AB T6H 4N1, Canada)

To study the effects of silence duration and frication frequency on the perception of stop consonants in /sCs/ context, a two-dimensional con-

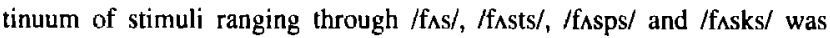
synthesized. Silence inserted within the $/ \mathrm{s} /$ frication ranged from $0-120 \mathrm{~ms}$ in 13 steps of $10 \mathrm{~ms}$, while the frication center frequency immediately surrounding the gap varied from $1500-4500 \mathrm{~Hz}$ in nine linearly spaced steps. Stimuli with a $0 \mathrm{~ms}$ gap had no change in overall amplitude near the insertion target, while stimuli in which frication frequency was held at $4500 \mathrm{~Hz}$ (that of $/ \mathrm{s} /$ ) had no change in spectral shape. Fifteen subjects were asked to categorize the stimuli as either fuss, fus-s, fusts, fusps or fusks. Subjects were told to respond with fus-s when a specific consonant was not perceived, even though two /s/ sounds could clearly be distinguished. Results from a logistic regression showed that both gap duration and the frication frequency immediately surrounding have a significant effect on categorizations of the three stop consonants $(p<0.0005)$. However, the linearity constraint imposed by a standard logistic regression appears to be too strong. Two other possibilities-quadratic categorical boundaries and hierarchical decision processes-are discussed as alternatives. [Work supported by SSHRC.]

2aSC5. Acoustic and perceptual study of French stop bursts: Implications for stop recognition. Linda Djezzar (CRIN-CNRS \& INRIA Lorraine, BP 239, 54506 Vandœuvre-les-Nancy Cedex, France)

A perceptual and acoustic investigation was conducted to better understand the discrimination power of the burst regarding place-of-articulation of French stops. These perceptual experiments showed that the burst provided very reliable spectral information about stop place and that prior knowledge of the vowel identity led to a slight but significant improvement in stop identification [A. Bonneau et al, to appear in J. Acoust. Soc. Am. (1995)]. This acoustic study confirmed that burst cues cannot be extracted and exploited without taking into account the vocalic context. Based on these conclusions, a hybrid recognizer was implemented. First, it recognizes the vocalic context, and then it takes this into account to extract and exploit the burst cues for the stop recognition task. The vocalic context was correctly recognized in $90 \%$ of the cases: Back vowels were perfectly recognized, central vowels with $80 \%$ accuracy, rounded front vowels with
$90 \%$, and unrounded front vowels with $89 \%$. The global recognition rate of stop place was $88 \%$. Statistical tests indicated that the best recognized stop was $/ \mathrm{k} /(93 \%)$, then $/ \mathrm{t} /(87 \%)$, and $/ \mathrm{p} /(84 \%)$. These results agree with listeners' performance observed in the perceptual experiments $(/ \mathrm{k} /: 94 \%$ $>/ \mathrm{V}: 91 \%>/ \mathrm{p} /: 62 \%)$.

2aSC6. Korean stops and afrricates: Acoustic and perceptual characteristics of the following vowel. Taehong Cho (Univ. of Texas, Arlington, TX 76019)

Despite numerous studies on lax, aspirated, and fortis stops in Korean, few have reported on the role of the following vowel in consonant perception. This paper examines the degree to which vowels convey the phonation characteristics of the preceding obstruents, with a hypothesis: Native speakers utilize vowel's quality in perceiving the preceding obstruents. This study includes (1) acoustic analyses of VOT, $F 0$, intensity build-up, and vowel length and (2) the perception test, where subjects were instructed to indentify CV syllables using stimuli with consonant portions completely removed. The acoustic analyses indicate that the acoustic characteristics associated with vowels pattern systematically with preceding obstruents (e.g., systematic difference in vowel length). In the perception test, subjects correctly identified the missing consonant $78 \%$ of the time, supporting the hypothesis. The probability was higher for the fortis than for the aspirated obstruents: Obstruents with longer VOTs spread less acoustic information into the following vowels, suggesting that the nature of VOT is possibly "voiceless vowelness." Most wrongly identified aspirated obstruents were misperceived as fortis ones, confirming that the aspirated and the fortis consonants share some acoustic features (e.g., higher $F 0$, rapid intensity buildup). The overall results indicate that following vowels play a pivotal role in consonant perception.

2aSC7. Production and perception of consonant coarticulation in Taiwanese. Shu-hui Peng (Dept. of Linguistics, Ohio State Univ., 222 Oxley Hall, 1712 Neil Ave., Columbus, OH 43210)

Taiwanese coda stop consonants are unreleased, so that their place of articulation tends to be confused with that of the initial consonant of any following syllable. This traditionally is described as place assimilationi.e., categorical feature change. Recent phonetic theories suggest an alternative description in terms of a continuous variation in degree of coarticulatory overlap between consonants. This study investigates the production and perception of coda consonants before different following onset consonants. Productions by six subjects were analyzed using electropalatographic (EPG) and acoustic measurements. Perception was examined using the concept formation paradigm. Preliminary data suggest that apparent place assimilation in Taiwanese is a noncategorical gestural coarticulation. The latency of the second gesture with respect to the first decreased as speech rate increased, so that gestural reduction was found in the production of every subject in normal and fast speech rates. The dental gesture was deleted more frequently than the velar gesture. In the perception test, the identification of coda place became worse as the latency of the second gesture decreased, so that the coda's gesture was overlapped more by the following onset gesture.

2aSC8. Aerodynamic evidence of preconsonantal stop lenition in Taiwanese. Chai-Shune K. Hsu (Dept. of Linguistics, Univ. of California at Los Angeles, 405 Hilgard Ave., Los Angeles, CA 90095-1543)

Taiwanese word-final stops lenite intervocalically. In addition, regressive place assimilation occurs optionally in casual speech. It has been suggested that gestural reduction is the crucial articulatory mechanism in place assimilation [J. Jun, UCLA Ph.D. dissertation (1995)]. Based on previously developed methods and interpretations [D. Silverman and J. Jun, Phonetica 51, 210-220 (1994)], oral pressure experiments were conducted to assess whether gestural reduction obtains before a stop consonant in Taiwanese. Data for all nonhomorganic consonant clusters containing a labial constituent were analyzed. Results of pressure measured behind the lips suggest that both oral and nasal word-final stops at all three places of articulation may reduce before a word-initial stop consonant. Reduction was found to be variable, but nonlabials reduce before labials more readily than labial reduction before nonlabials. Variable gestural overlap was also detected in consonant clusters. Word-final gestural reduction is suggested 
to be a general process, occurring both intervocalically and preconsonantally, but perceived as "lenition" intervocalically and "assimilation" preconsonantally.

2aSC9. Techniques for improved stop and nasal consonant discrimination. Philipos C. Loizou (Dept. of Elec. Eng., Arizona State Univ., Tempe, AZ 85287-7206), Michael F. Dorman (Arizona State Univ., Tempe, AZ 85287-0102), and Andreas S. Spanias (Arizona State Univ., Tempe, AZ 85287-7206)

The automatic recognition of stop and nasal consonants is known to be a difficult recognition task. This paper presents various techniques that can be used to improve the discrimination of stop and nasal consonants. An improved spectral representation for stop consonants is proposed, which unlike other feature representations, emphasizes the mid-to-high regions of the spectrum. A subspace projection approach, which is used as a preprocessing step in a hidden Markov model based system, is also proposed for improved nasal discrimination. This approach finds a transformation matrix which maps the original nasal observation onto a subspace such that the "distance" between the nasals is maximized on the subspace. Two statistical distance measures are investigated for finding the transformation matrix, namely the divergence and the Bhattacharyya measures. Results on stop and nasal consonant recognition will be presented using the subspace approach and the improved spectral stop representation.

2aSC10. Acoustic cues for / $/ \boldsymbol{l} /$ in American English. Nicholas Kibre (Speech Technol. Lab., Panasonic Technologies, Inc., 3888 State St., Santa Barbara, CA 93105 and Dept. of Linguistics, Univ. of California, Santa Barbara) and Kazue Hata (Panasonic Technologies, Inc., Santa Barbara, CA 93105)

Distinguishing between the voiceless fricatives $/ f /$ and $/ \theta /$ is a difficult problem in natural and synthetic speech. In a previous experiment using natural stimuli [K. Hata et al., Proc. ICSLP 327-330 (1994)], it was found that adding vowel transitions increased identification for $/ f /$ at least $15 \%$ in comparison with frication-only stimuli. However, with vowel transitions, the identification of $|\theta|$ failed to show significant improvement. The purpose of the current study was to investigate, with an improved procedure, significant cues for $/ \theta /$ which we can use in our synthesizer. Six monosyllabic nonsense words (e.g., /fiyk/, $/ \theta \mathrm{ayk} /$ ) were recorded. Segments of approximately $30-\mathrm{ms}$ duration from different locations of $|\theta|$ and its following vowel were spliced into f-initial words. Eight subjects were asked to identify each stimulus as "th," " $\mathrm{f}$ " or "indistinguishable." In the /iy/ context, /f/-initial stimuli spliced with fricative-vowel transitions from $|\theta|$ were perceived as $|\theta| 55 \%$ of the time, while stimuli involving other vowel contexts and other splices tended to be perceived as $/ f /$ This implies that a cue for $|\theta|$ resides in this transition when followed by a high, front vowel, but that the cue is rather obscure in the $0-5 \mathrm{kHz}$ region when other vowels follow.

2aSC11. Clicks in a Chinese nursery rhyme. Geoffrey S. Nathan (Dept. of Linguistics, Southern Illinois Univ., Carbondale, IL 62901-4517)

Clicks (speech sounds produced by velaric suction) have been reported in a number of African languages but have never been reported elsewhere in the world. However, several Chinese dialects have been discovered to use clicks in a special children's version of a traditional nursery rhyme. This paper reports on the nasalized palatal clicks found in two different renditions of this nursery rhyme. The clicks replace normally occurring syllable-initial velar nasals in this special version, which apparently functions as a tongue-twister in those dialects that display this behavior. The clicks are completely integrated within the syllable structure of the words that they occur in, and sound quite similar to those found in Xhosa and !Kung. The paper will present articulatory descriptions and acoustic analyses of the clicks, and there will be an audio tape available for those who wish to hear the data. Some speculation on the origins and functions of the clicks will be presented.
2aSC12. Individual differences and the acquisition of new phonetic categories. Pamela Case and Betty Tuller (Ctr. for Complex Systems and Dept. of Psych., Florida Atlantic Univ., Boca Raton, FL 33431-0991)

Adults' acquisition of non-native phonetic categories occurs within the context of an individual's existing phonology. The present work examines (1) the process of acquiring a new phonetic category, (2) the impact of the new phonetic category on nearby, previously existing categories, and (3) transfer of learning to novel contexts. Monolingual American English speakers were required to learn the Hindi voiced, unaspirated, dental stop consonant. First, listeners were asked to identify and judge the goodness of stimuli on two synthetic continua spanning a range from Hindi dental to American English alveolar stop consonants. One continuum was voiced, the other voiceless. In addition, listeners judged the similarities between all possible pairs of stimuli on each continuum. After this perceptual mapping procedure, subjects participated in a two-altemative, forced-choice training program using only voiced stimuli. Progress was monitored throughout training. Following training, the mapping procedure was repeated with both the voiced and voiceless continua. Results are discussed from the theoretical perspective of the nonlinear dynamical approach to learning and transfer in motor behavior presented by Zanone and Kelso [in Swinnen, Heuer, Massion, \& Casaer, 461-490 (1994)] and with respect to Best's perceptual assimilation model and Kuhl's perceptual magnet effect. [Work supported by NIDCD and NIMH.]

2aSC13. Improvements in the perception of American English vowels by Brazilian bilinguals. Marialice Szpigel (Dept. Speech, Communication Sciences, \& Theatre, St. John's Univ., Jamaica, NY 11439) and Fredericka Bell-Berti (St. John's Univ., Jamaica, NY 11439, and Haskins Labs., New Haven, CT 06511)

Brazilians who are fluent in English have more difficulty perceiving American English vowels than consonants. This study examines vowel perception by eight native speakers of Brazilian Portuguese at the beginning and again at the end of their sixth year of study of American English (at the Instituto Brasil-Estados Unidos, Rio de Janeiro, Brazil). At this level, all students are able to speak English fluently, but with varying degrees of proficiency; the subjects had different levels of English proficiency at the beginning of the study. Their perceptions of American English vowels (produced by a native speaker of American English) will be compared at the beginning and end of the study, and will also be compared to the reported perception of American English vowels [G. Peterson and $\mathrm{H}$. Barney, J. Acoust. Soc. Am. 39, 151-184 (1952)]. Changes in the students' productions of seven American English vowels over the course of the year will also be examined. [Work supported by St. John's University and by NIH Grant DC-00121 to the Haskins Laboratories.]

2aSC14. English $/ r /$ and $/ /$ production in American and bilingual Japanese subjects. Walter R. Naito (Haskins Labs., 270 Crown St., New Haven, CT 06511)

The tongue shapes of American and bilingual Japanese subjects were evaluated when producing words that include $/ \mathrm{r} / \mathrm{s}$ and $/ \mathrm{l} / \mathrm{s}$ in English. An electromagnetic midsagittal articulometer (EMMA) system was utilized to observe and record the tongue shape. A total of six transducer coils was put on the tongue. Four were on the superior side of the tongue, and two were on the inferior side. The tongue shapes of the American subjects when producing / $r$ 's generally agreed with the earlier studies done by Delatree and Freeman [Linguistics 44, 28-68 (1968)]. On the other hand, the Japanese subjects had more uniform tongue shapes across subjects, which was thought to correlate with the Japanese /r/'s. [Work supported by NIH.]

2aSC15. Perception and production of the English $/ r /-f /$ contrast by Japanese speakers: Relationship between performance and learning in the two domains. Ann R. Bradlow (Speech Res. Lab., Dept. of Psych., Indiana Univ., Bloomington, IN 47405), Reiko A. Yamada (ATR Human Information Processing Res. Labs., Soraku, Kyoto, 619-02, Japan), David B. Pisoni (Indiana Univ., Bloomington, IN 47405), and Yoh'ichi Tohkura (ATR Human Information Processing Res. Labs., Soraku, Kyoto, 619-02, Japan)

This study investigated the relationship between performance in perception and production, and the relationship between degrees of learning in the two domains within individual subjects. Eleven monolingual Japanese 
adults participated in an $/ r /-\Omega /$ perceptual training program. Both perception data (minimal pair identification scores) and production data (recordings of $/ r /-/ / /$ minimal pair productions) were collected before and after perceptual training. Pre- and post-test production data were then evaluated by native speakers of American English in a minimal pair identification task. Results showed considerable individual variation in all aspects of the perception-production relationship. Subjects varied widely in pretest levels of performance in both perception and production, as well as in improvement in perception and production. In general, subjects who performed well in the perception pretest also had good productions at pretest; however, subjects who were low performers in the perception pretest varied in their production abilities at pretest. Additionally, there was considerable variation in the transfer of perceptual learning to production. Subjects with a relatively high degree of improvement in perception did not necessarily have a comparable degree of improvement in production. Possible sources of these individual differences, and implications for the perception-production link will be discussed.

2aSC16. Phrase-final lengthening and stress-timed shortening effects in native speakers and Japanese learners of English. Motoko Ueyama (Dept. of TESL/Appl. Linguistics, Univ. of Califomia, Los Angeles, CA 90024)

This study analyzes the durational patterns of native Japanese speakers learning English, with a focus on the two major prosodic effects: phrasefinal lengthening and stress-timed shortening. To investigate the significance of these effects, a production experiment was conducted, adapting the method of Beckman and Edwards [Papers in Laboratory Phonology I, 152-178 (1990)]. Native speakers, beginning Japanese learners, and advanced Japanese learners of English were compared. Results are as follows: (1) The phrase-final lengthening effect is large and the stress-timed shortening effect is small in the speech of the native English speakers and the advanced Japanese learners; (2) all Japanese speakers are more successful in applying phrase-final lengthening before larger prosodic boundaries than before smaller ones. Further analysis shows that the native English speakers make more durational contrast between lexically stressed and unstressed syllables (this is called local contrast) and also differentiate the three degrees of boundary strength hierarchically (this is called hierarchical contrast). These two types of durational contrasts are considered to be effective benchmarks to assess the acquisition of English stresstiming by Japanese learners, due to their considerable correlation with learner proficiency levels.

2aSC17. English vowels for Chinese speakers enrolled in English as a second language classes. Therese I. Huber, Hong Wei Dou, Emest M. Weiler, and Joseph G. Agnello (MI \#379, Speech Sci. Lab., Communication Sci., Univ. of Cincinnati, Cincinnati, $\mathrm{OH} 45221$ )

Chinese and English differ considerably in the characteristics of the vowels. The present study focuses on acoustic characteristics of Chinese production of English vowels. The seven Chinese students in the present study had all been bom in mainland China, and were students at the University of Cincinnati. All were enrolled in ESL (English as a second language) classes because of difficulties with English pronunciation. Two native English speakers were used to provide a comparative analysis, by minimal pairs, for formant placement of the vowels $/ \mathrm{i} /, / \mathrm{V}$, and others. A total of five English speakers phonetically transcribed the productions and tallied the data into a confusion matrix. The acoustic analysis, performed separately by five speech science students revealed systematic differences in the production of the vowels associated with the confusion. The Chinese first formant was lower and the second was higher than found for the midwestern English speakers. Insufficient reduction in frequency between minimal pairs was the source of auditory confusion. The application to ESL speech therapy will be discussed.
2aSC18. Intelligibility assessment of Chinese-accented English. Catherine L. Rogers (Commun. Disord. Technol., Inc., 205 S. Walnut St., Bloomington, IN 47404 and Depts. of Linguistics and Cognitive Sci., Indiana Univ., Bloomington, IN) and Jonathan M. Dalby (Commun. Disord. Technol., Inc., Bloomington, IN 47404)

Although it is generally accepted that a strong foreign accent renders a speaker less intelligible to native listeners, few studies have attempted to investigate specific sources of this deficit. The present study explores the diagnostic effectiveness of a minimal-pairs test of intelligibility. An inventory of phonemic errors was compiled from careful transcriptions of the spoken English of two native speakers of Mandarin Chinese. Minimal pairs were constructed for each error, using the intended phoneme and the closest English phoneme transcribed. Eight additional native speakers of Mandarin Chinese were recorded reading the target words in the minimalpairs list and a set of 20 sentences. The minimal-pair target words were presented to groups of native listeners in a forced-choice task; in a second task, listeners were presented with the sentences and asked to write down what they understood. Preliminary results from listener groups for three of the speakers demonstrate (1) no significant differences across listener groups for a control speaker, indicating test reliability, (2) significant differences across listener groups for the three speakers, indicating test sensitivity, and (3) percent-correct scores on the minimal-pairs test are predictive of percent of words correctly identified in the sentence-listening task. [Work supported by NIH-NIDCD Grant \#2R44DC02213.]

2aSC19. A phonetic study of stress in Korean. Sun-Ah Jun (Dept. of Linguistics, Univ. of California, 405 Hilgard Ave., Los Angeles, CA 90024-1543)

Stress in Korean (Seoul) has been controversial for many years: Some linguists believe there is word-level stress and others do not. Among those who believe that word-level stress exists, there has been controversy regarding its location: the first or second syllable (H. Lee, 1973), the second syllable (Huh, 1985), or the final syllable (Choi, 1935). Even though it has been shown that there is no fixed acoustic property of stress, researchers have found that stress is detectable based on duration, amplitude and $F 0$ [Fry (1958), Lea (1977), Beckman (1986)]. By examining these prosodic features, this paper investigates whether there is stress in Korean and, if so, what its domain and location are. Words with a reiterated syllable, /na/ or $/ \mathrm{ta}$, were uttered by native Seoul speakers in different prosodic positions. The preliminary results show that Korean stress is not a word-level but a phrase-level stress: The location of the stressed syllable depends on its position in an accentual phrase (Jun, 1993). In addition, the stress falls on either the first or the second syllable of the phrase depending on the number of syllables in the phrase, syllable weight, and the position of the phrase in the sentence.

2aSC20. Acoustic properties of primary and secondary word-level stress in Estonian. Matthew K. Gordon (Dept. of Linguistics, UCLA, 405 Hilgard Ave., Los Angeles, CA 90024)

Languages differ in the way they signal stress. In Estonian, the primary stressed syllable is marked by increased onset duration [Lehiste, Consonant Quantity and Phonological Units in Estonian (Indiana Univ., Bloomington, 1966)] and by a pitch rise [Liiv, Sovetskoe Finno-Ugrovedenie 21(1). 1-13 (1985)]. The acoustic properties of secondary stress are subtle, resulting in controversy over its location [Hint, Eesti Keele Sônafonoloogia (Eesti NSV Teaduste Akadeemia, Tallinn, 1973); Eek, EPP, 20-59 (1982)]. To determine further the acoustic properties of Estonian stress, amplitude, $F 0$, and consonant and vowel durations were measured for di-, tri-, and tetrasyllabic words. Results for primary stress conformed to those found by Lehiste and Liiv. Secondary stress was characterized by an interruption in the $F 0$ decline (Eek, 1982) and by lengthening of the onset, but not the rhyme. Estonian is thus unusual in signaling stress by pitch and by lengthening of the onset, but not the rhyme. Unstressed open syllables were significantly longer after primary stressed syllables of both the $\mathrm{CV}$ [Lehiste, Language 41(3), 447-456 (1965)] and the CVV type, than after CVC, arguing against foot isochrony [Eek and Remmel, Speech Communication Seminar, 179-185 (1974)]. Implications of these phonetic measurements for proposed algorithms of stress assignment in Estonian will be considered. 
2aSC21. Modeling the articulatory dynamics of two kinds of stress. K. Bretonnel Cohen, Mary E. Beckman (Dept. of Linguistics, Ohio State Univ., 1712 Neil Ave., Columbus, OH 43210-1298), Jan Edwards, and Marios Fourakis (Ohio State Univ., Columbus, $\mathrm{OH} 43210$ )

A study reported at an earlier meeting of this Society examined finegrained timing cues to three levels of stress by comparing jaw kinematics in intonationally accented syllables (with full vowels), unaccented syllables (with full vowels), and completely stressless (reduced-vowel) syllables. For all four speakers in the study, measured values for movement duration, displacement, and peak velocity were consistently largest in the accented syllables and smallest in the reduced-vowel syllables. This study examines the relationships among these kinematic measures using two different models of the underlying gestural dynamics. The first generated shorter (less stressed) syllables by decreasing the latency of the closing gesture relative to the opening gesture without changing the targeted gestural speed or displacement. The second generated shorter syllables by changing the targeted gestural speed, but decreasing targeted displacement more, so as not to increase the predicted velocities. The first model generated predicted durations, which were closer to the observed distribution of durations among the three stress types, whereas the second generated predicted displacements that were closer to the observed distribution of displacements. Neither generated the observed distribution of velocities, suggesting that a hybrid model is necessary.

2aSC22. Timing differences between prenuclear and nuclear pitch accents in Italian. Mariapaola D'Imperio (Dept. of Linguistics, The Ohio State Univ., Oxley Hall, 1712 Neil Ave., Columbus, OH 43210-1298)

In Italian, as in English, lexically stressed syllables can receive a pitch accent, with the last accented syllable being the most prominent (i.e. "sentence stress"). Recent analyses based on data from northern Italian varieties describe the nuclear accent of the neutral declarative contour as falling onto the stressed syllable $\left(\mathrm{H}+\mathrm{L}^{*}\right)$, while the prenuclear one, presenting a comparatively later peak, is described as qualitatively different from the other $\left(\mathrm{H}^{*}\right)$. This analysis is problematic since there is no meaning contrast between $\mathrm{H}^{*}$ and $\mathrm{H}+\mathrm{L}^{*}$. An alternative analysis of the timing differences models the shape of both accents as $\mathrm{H}^{*}$, where the high in the nuclear one is retracted due to an upcoming low tone associated with the remaining of the utterance (tonal repulsion hypothesis). A similar account has been proposed for English $\mathrm{H}^{*}$ by Silverman and Pierrehumbert. This analysis predicts that the timing differences will depend on such things as the number of following stressed syllables for the L-, and also that other pitch accent types (such as the $L^{*}+H$ of the question) will show comparable differences. The analysis was tested by measuring the location of the fundamental frequency peak relative to the beginning of the stressed vowel in various prosodic contexts.

2aSC23. Identification of rhythmic forms of speech production. Fred Cummins (Depts. of Linguistics and Cognitive Sci., Indiana Univ., Bloomington, IN 47405)

An experiment was conducted to elicit stable forms of thythm in spoken English. Stimuli were constructed, each of which consisted of a repeated series of the words take and cards. While the interval from one take to the next was a constant $(1.5 \mathrm{~s})$, the timing of cards relative to this interval was systematically varied. Subjects were instructed to repeat the phrase take a pack of cards, so that the relative timing of take and cards matched that of the stimulus. After some time, the stimulus was switched off, and subjects attempted to maintain the prescribed timing pattern on their own. Although there were eight target timing patterns in the stimuli, the obtained distribution of subjects' productions was trimodal rather than octomodal. The three modes are hypothesized to comespond to three stable, preferred forms of rhythmic production for this phrase. These data strongly suggest that there is an observable rhythmic basis to English speech production. [Supported by ONR.]

2aSC24. Internal temporal structure of Mandarin syllables. Alan Bell (Dept. of Linguistics, Univ. of Colorado, Boulder, CO 80309) and Mei-Chun Liu (Natl. Chiautung Univ., Taiwan)

The durational interactions among different combinations of onsets, nuclei, and codas in Mandarin Chinese syllables are examined, based on data from six speakers at normal and fast rates of speech. This report extends and refines preliminary results, based on four speakers at a normal tempo, that were reported earlier [A. Bell and M. Liu, J. Acoust. Soc. Am. Suppl. 1 87, S67 (1990)]. Effects of different onsets (In t ft ), vowels ( $($ a $\mathrm{i}$ ul), diphthongs ( (ai au ou ia iaul), and nasal codas ( $\ln \mathrm{g} \backslash$ ) were considered. Consistent effects of onsets, vowel height, complex nuclei, and nasal codas on both syllable and rhyme durations were found. These effects, however, were relatively small, of the order of $15 \%$ for rhyme durations, and from about $5 \%$ to $10 \%$ for syllables, apparently consistent with a hypothesis that Mandarin rhymes and syllables are perceptually invariant. On the other hand, the nature of segment compensations was more consistent with segment-local and universal mechanisms than with language-particular global timing units of rhyme or syllable. [Work supported by the University of Colorado Council on Research and Creative Work.]

2aSC25. Organization of acoustic singularities in running speech. Charles Andrew Harsin and Kerry P. Green (Natl. Ctr. for Neurogenic Commun. Disord., Tucson, AZ 85721)

Investigating speech rhythm by examining an utterance's syllabic-beat organization seems natural, but is problematic. An utterance must first be divided into syllables, then each syllable's beat must be located through mathematical modeling or the collection of subjects' judgments. This study approaches the problem in a more straightforward way by examining the organization of singularities, specifically acoustic envelope amplitude and amplitude rate-of-change maxima, in the acoustic speech wave. These acoustic features are the basis for some acoustic models of syllabic beat location, and so are plausible candidates for more directly measurable correlates of speech rhythm. So far, qualitative analyses suggest that these acoustic features are organized in a quasiperiodic manner which is neither strictly periodicity nor random and that is similar to the organization found in the behaviors of other physical and biological systems. Quantitative analyses will be discussed that further specify the organization of the data. [Work supported by National Multipurpose Research and Training Center Grant DC-01409 from the National Institute on Deafness and Other Communication Disorders.]

2aSC26. Young infants' use of prosodic cues to assess lexical information. Francisco P. Lacerda (Inst. of Linguistics, Stockholm Univ., S-106 91 Stockholm, Sweden)

Young infants were tested by the high-amplitude sucking (HAS) technique to assess their ability to use prosodic information to pick up word contrasts occurring in sentences. The results presented here were obtained from 33 experiments in which 20 Swedish infants ( 8 girls and 12 boys) participated. The ages at the date of the experiments varied between 58 and 147 days (mean age 105 days, median 108 days). The infants listened to pairs of natural carrier sentences, produced as child-directed speech, in which target words had been inserted. The infants were randomly assigned to each of the four conditions: (1) contrasting target words in focal position, (2) contrasting target words in nonfocal position, (3) contrast only in the position of the sentence focus, and (4) control condition (no change). The results seem to suggest that the infants, while sensitive to displacement of the sentence focus $[F(1,29)=4.518, p<0.042]$, are unable to attend to the word contrast when the target word is in emphatic position $[F(1,29)=2.358, p<0.135]$, but perform well when the same target word contrasts are presented in nonfocal position $[F(1,29)=8.143, p<0.008]$. [Research supported by The Bank of Sweden Tercentenary Foundation, Grant 94-0435.]

2aSC27. Inclusion of a prosodic module in spoken language translation systems. Robert Eklund and Bertil Lyberg (Telia Res. AB, Systems Res., Spoken Language Processing, S-136 80 Haninge, Sweden)

Current speech recognition systems mainly work on statistical bases and make no use of information signaled by prosody, i.e. the segment duration and fundamental frequency contour of the speech signal. In more advanced applications for speech recognition, such as speech-to-speech translation systems, it is necessary to include the linguistic information conveyed by prosody. Earlier research has shown that prosody conveys information at syntactic, semantic, and pragmatic levels. The degree of linguistic information conveyed by prosody varies between languages, from languages such as English, with a relatively low degree of prosodic 
disambiguation, via tone-accent languages such as Swedish, to pure-tone languages. The inclusion of a prosodic module in speech translation systems is not only vital in order to link the source language to the target language, but could also be used to enhance speech recognition proper. Besides syntactic and semantic information, properties such as dialect, sociolect, sex, and attitude, etc. is signaled by prosody. Speech-to-speech recognition systems that will not transfer this type of information will be of limited value for person-to-person communication. A tentative architecture for the inclusion of a prosodic module in a speech-to-speech translation system is presented.

2aSC28. The relationship between prosody and syntax in generation and comprehension of spoken messages. Hiroya Fujisaki and Sumio Ohno (Dept. of Appl. Electron., Sci. Univ. of Tokyo, 2641 Yamazaki, Noda, 278 Japan)

While it is generally believed that prosody of a spoken message conveys information concerning its syntactic structure, there exist many cases where prosody and syntax are discordant. The discordance, however, does not usually cause any difficulty in comprehension. The present study aims at elucidating the process whereby the prosodic structure is determined in speech production, as well as the process whereby it is utilized in speech perception. Using the contour of the voice fundamental frequency as an index, this paper shows examples of such discordance in utterances of various languages including English, Japanese, and Chinese, and presents an interpretation for the origin of the discordance, based on a model of the cognitive processes involved in message generation and speech produc- tion. The role of such discordance in speech preception and message comprehension is also discussed, referring to the cognitive processes involved. Finally, the implication of these results for speech synthesis by rule and automatic speech understanding is also mentioned.

2aSC29. On the acoustics of broad and narrow focus. Steven $R$. Hoskins (Univ. of Delaware, Newark, DE 19711)

Linguistic analyses of focus [Halliday (1967), Chomsky (1971), Ladd (1980), Selkirk (1984), Gussenhoven (1984)] state that under certain conditions, foci of different scope have identical prosodic realizations. These claims have not yet been supported by empirical data. Eady et al. [Lang. Speech 29(3), 233-251 (1986)] found durational differences between broad and narrow focus within the verb phrase (VP). However, these experiments did not control for syntactic structure: According to Selkirk (1984), only verb-argument VPs have identical broad/narrow focus realization; verb-adjunct VPs do not. This study directly investigates the interaction of focus with verbal arguments and adjuncts. An experiment was conducted where matched sentences with verb-argument and verb-adjunct structures were read under three focus conditions: broad (on VP), narrow (on verb), narrow (on postverbal argument/adjunct). Both speech and electroglottographic data were gathered. Durations, absolute pitch, and change in pitch on the stressed syllable of the verb were analyzed. Preliminary results (three subjects) support Selkirk (1984): In the verb-adjunct structures, focus on the VP and narrow (postverbal) focus are significantly different for duration and pitch change; verb-argument structures, however, are not significantly different for these conditions.

Session 2aUW

\title{
Underwater Acoustics: Spatial, Temporal and Frequency Dispersion Due to Boundary Scattering in Shallow Water Propagation I
}

\author{
Peter H. Dahl, Cochair \\ Applied Physics Laboratory, College of Ocean and Fisheries Sciences, University of Washington, 1013 N.E. 40th Street, \\ Seattle, Washington 98105
}

Paul C. Hines, Cochair

Defence Research Establishment Atlantic, P.O. Box 1012, Dartmouth, Nova Scotia B2Y 3Z7, Canada

Chair's Introduction-8:05

Invited Papers

8:10

2aUW1. Scattering function characterization of time and frequency spreading in shallow water propagation. Peter G. Cable (BBN Systems and Technologies, Union Station, New London, CT 06320)

Under certain conditions the multipath time delay and frequency spread of signals propagating in shallow water can be described usefully with the ocean modeled as a randomly time-varying linear filter. Starting with an explicit physical model for the time-varying transfer function of a shallow water channel, an alternative system function, the delay-Doppler spread function, can be defined and its autocorrelation determined. A particularly simple form of the spread function autocorrelation, the scattering function, results when the propagation multipaths are uncorrelated and locally stationary in the wide sense. When the scattering function description is valid, signal propagation input-output relations can be expressed in terms of the medium scattering function and the input signal ambiguity function. The scattering function formulation will be reviewed and shown to be most useful for channels supporting many boundary- 
interacting paths (e.g., shallow water) at mid or high frequency. Using the scattering function the influence of the medium on different sonar signal types will be demonstrated and methods for measuring channel characteristics indicated. Channel characteristics determined from data obtained during ACT II on the New Jersey Shelf under downward refracting propagation conditions will be presented and discussed in terms of the scattering function description. [Work supported in part by ARPA.]

\section{8:35}

2aUW2. Theoretical approaches for computing FAT spreads. Diana F. McCammon (Appl. Res. Lab. and the Graduate Prog. in Acoust., Penn State Univ., P.O. Box 30, State College, PA 16804)

FAT (frequency, angle, and time) spreading is approached theoretically through the Helmholtz integral equation for scattering from random rough surfaces. Using the Kirchhoff approximation, angle spread is readily found to be related to the probability of surface slopes. Various approximate evaluations are discussed for modeling time spread, from numerical ray tracing to integrals over pulse shapes. Solving for the half-power points of the intensity shows the link between angle and time spread. Frequency spread functions are discussed, illustrating the difficulty of using Fresnel phase approximations for higher-order corrections to the Doppler shift.

\section{Contributed Papers}

\section{9:00}

\begin{abstract}
2aUW3. Measurements and interpretation of spatial coherence and angular spread resulting from multiple boundary interactions in a shallow-water channel. Peter H. Dahl and Warren L. J. Fox (Appl. Phys. Lab., Univ. of Washington, Seattle, WA 98195)
\end{abstract}

Measurements of the spatial coherence of high-frequency [0(10 kHz)] sound having propagated in a shallow-water channel are discussed. The experiment was conducted near Key West, Florida, in water depth of approximately $25 \mathrm{~m}$. The bottom consisted of calcium shell deposits and soft mud. Measurements were made using omnidirectional sources suspended from a spar buoy and a horizontal line array of receivers. Broadband pulses were used in order to resolve the separate arrivals, e.g., direct path, singlesurface, and double-surface bounce paths. With each boundary interaction, either surface or bottom, the forward propagating energy is scattered further in angle. The transmissions were measured along a line array oriented transverse to the direction of propagation, thereby giving an estimate of the horizontal coherence or equivalent horizontal angular spread. The results are discussed in the context of key geometric parameters such as source/ receiver depth and range, and environmental parameters such as wind speed and surface wave spectral characteristics. [Work supported by ONR.]

\section{9:15}

2aUW4. On the dependence of bottom scatter on frequency, grazing angle, and azimuth. Tokuo Yamamoto (Appl. Marine Phys. Div., RSMAS, Univ. of Miami, 4600 Rickenbacker Cswy., Miami, FL 33149)

The bottom scatter data from many different shallow and deep water sites by Jackson and Briggs (1992), Ogden and Erskine (1993), Hines and Barry (1992), and others show wide varieties of dependencies on frequency, grazing angle, and azimuth. Model predictions so far have not been satisfactory. For example, Mourad and Jackson (1993) suggested that the frequency dependence of bottom backscatter is due to the vertical velocity gradient. However, unrealistic velocity gradients were needed to predict the backscatter increasing with frequency. The high resolution crosswell tomography and cores of seafloor sediments show that the threedimensional (3-D) spectra of velocity and density fluctuations within the sediments are anisotropic and dipping [Yamamoto, J. Acoust. Soc. Am. (to be published)]. It will be shown in this paper that the various dependencies of bottom backscatter on frequency, grazing angle, and azimuth observed in the data are results of the 3-D spectra of density and velocity fluctuations in sediments based on a volume scattering theory by Yamamoto [J. Acoust. Soc. Am. (in press)]. [Work supported by ONR.]
9:30

2aUW5. Shallow water measurements of time spreading at high frequency. Paul C. Hines Arthur, J. Collier, and James A. Theriault (Defence Res. Establishment Atlantic, P.O. Box 1012, Dartmouth, NS B2Y 3Z7, Canada)

Time spreading measurements provide an indirect measure of the acoustic bandwidth that can be supported by the water channel, which is critical to the design of sonar systems. Time spreading measurements were collected in a water channel $100 \mathrm{~m}$ deep, off the coast of Nova Scotia. Data were collected at frequencies of $20-22 \mathrm{kHz}, 27-29 \mathrm{kHz}$, and $35-37 \mathrm{kHz}$ using linear FM pulses $2 \mathrm{~s}$ in duration. The experiments were part of a collaborative trial conducted by Canada, the US, and the UK. Canada's Seahorse array, an anchored, high-frequency active sonar was employed for the source-receiver, and a UK free drifting echo repeater was employed for the target. Source-receiver and target position were recorded using a portable target range operated by the US. In the paper, time spreading measurements are compared with estimates obtained from the generic sonar model (GSM) for the experiment geometry. The GSM estimates of time spreading due to multipath propagtion compare favorably with the experimental data. However, time spreading of individual paths beyond that predicted by GSM is also evident.

\section{9:45}

2aUW6. A coupled bispectral, temporal and spatial coherence function of the pressure field, scattered from a moving sea surface. Christian Bjernum-Niese and Leif Bjornd (Dept. of Industrial Acoust., Tech. Univ. of Denmark, Lyngby, Denmark)

Fluctuations in the scattering of high-frequency sound from the moving sea surface is of significance, particularly in underwater acoustic communication systems using adaptive methods. Surface scattering may statistically be described using coherence functions, especially for higher frequencies when the coherent part of the pressure field is virtually nonexistent. Previous studies have presented coherence functions as a function of either spatial and temporal variations of the channel, but with a fixed signal carrier frequency or two signal frequencies and temporal channel variations (or some of the several possible Fourier transform duals). Here, a coupled bispectral, temporal and spatial coherence function is presented. The coherence function is derived from the pressure field, given by the two-dimensional Kirchhoff-Helmholtz integral for two monochromatic tones evaluated at separate receiver positions. The channel variations are caused by a wind-driven, gravity-wave dispersed sea surface with a Pierson-Moskowitz spectrum. The derivation of the coherence function involves numerical integration. Numerical results are compared to earlier model data from the literature. [Work sponsored by the Danish Technical Research Council.] 
10:00-10:15 Break

\section{0:15}

2aUW7. Real time signal processing for high-frequency long distance crosswell tomography. Murat Kuru and Tokuo Yamamoto (Geoacoustics Lab., RSMAS, Univ. of Miami, 4600 Rickenbacker Cswy., Miami, FL 33149)

Acoustic crosswell tomography was used for determining the sediment porosity, permeability, and shear strength from travel-time measurements by means of travel-time inversion and rock mechanics [Yamamoto $e t$ al. Geophysics (1994) (1995)]. These properties can be used to model the propagation and scattering of acoustic waves in saturated sediments. Due to the effects of intrinsic attenuation, transmission loss, and scattering, crosswell measurements usually are limited to ranges of 50-100 times the wavelength of the signals. It was believed that the diffusive nature of scattering put the coherent limit of acoustic wave propagation in sediments at approximately 1000 wavelengths. To extend the measurements of the propagation of acoustic waves to longer ranges, a real time processing and data acquisition system was employed. A crosswell tomography experiment with a crosswell distance of $540 \mathrm{~m}$ was performed at a limestone aquifer at Sanibel Island, Florida. Pseudorandom binary sequences modulated with carrier frequencies up to $6 \mathrm{kHz}$, which puts the range around 1400 wavelengths, were successfully transmitted by using pulse compression and coherent averaging techniques. The hydraulic structure of the Earth with a cross section of $540 \mathrm{~m} \times 150 \mathrm{~m}$ has been imaged at spatial resolution of a few meters. [Work supported by ONR.]

\section{$10: 30$}

2aUW8. Frequency dependence of sound propagation in shallow water: Theory and experiments. Mohsen Badiey (Graduate College of Marine Studies, Univ. of Delaware, Newark, DE 19716), Kevin P. Bongiovanni (Rensselaer Polytechnic Inst., Troy, NY 12180), Indra Jaya (Univ. of Delaware, Newark, DE 19716), and William L. Siegmann (Rensselaer Polytechnic Inst., Troy, NY 12180)

Experimental observations of broadband acoustic propagation in a known geological region of the Atlantic Generating Station (AGS) site [Badiey et al., J. Acoust. Soc. Am. 96, 3593-3604 (1994)] has prompted new approaches to understanding frequency-dependent behavior in shallow-water regions. First, recent acoustic observations and detailed geological borehole measurements are reviewed, along with a threedimensional model of the geoacoustic data that have been developed using the kriging method. Parabolic equation modeling, including rangedependent sound speed and attenuation, is performed for both $\mathrm{cw}$ and broadband signals in this region. This is accompanied by normal mode investigations in which trapped modes in the layered media and rangedependent mode coupling are examined. A modal-based theory is presented to explain quantitatively the interference patterns observed in the experimental data (transmission loss versus frequency) in terms of waveguide parameters. It is shown how layered shallow-water waveguides act as bandpass filters in which broadband acoustic energy is selectively broken into narrow-band components, thereby providing new insights applicable to existing inverse techniques.

\section{0:45}

2aUW9. Broadband frequency dispersion in shallow water as an active classification technique. James H. Wilson ${ }^{\text {a) }}$ (Naval Postgrad. School, Dept. of Oceanogr., Monterey, CA 93943), J. Huw Davies, ${ }^{\text {b) }}$ and Robert H. Bourke (Naval Postgrad. School, Monterey, CA 93943)

Recently, an active broadband dispersion phenomenon was observed empirically in measured data for propagation paths within the mixed laye (ML) [Dien et al. (1994)]. This so-called Wilson Dispersion Phenomena (WDP) is explained simply by noting that high-frequency energy trapped within the ML travels faster than low frequencies that diffractively "leak" out of the ML. Thus WDP is a useful technique for distinguishing between reflectors in the ML and reflectors below the ML. More recently it was shown that the WDP may occur in other shallow-water environments [Davies et al. (1994)]. Several sound-speed profile (SSP) environments are examined, using normal mode theory, for depth dependence of broadband frequency dispersion. A very conservative approach is taken regarding frequency reflectors. Geoacoustic properties of the bottom are not addressed. SSPs for which dispersion depth dependence is observed within the water column for a lossy bottom are labeled useful for active classification. Future research including geoacoustic properties of the sub-bottom may lead to a wider range of SSP and geoacoustic sub-bottom environments which produce dispersion depth dependence. It has been empirically observed that bottom reflectors (the primary false targets) never have significant dispersion and are spread over much longer times than reflectors within the water column. ${ }^{\text {g) }}$ Work performed while on temporary leave from Neptune Sciences, Inc., Slidell, LA 70458; ${ }^{\text {b) }}$ Currently stationed at Fleet Numerical Oceanographic Ctr., Monterey, CA 93943.

\section{1:00}

2aUW10. Scattering function characterization of underwater acoustic channels. Christopher J. Link and R. Lee Culver (Graduate Prog. in Acoust., Penn State Univ., State College, PA 16804)

Underwater acoustic channels can be modeled as linear, time-varying, random filters. If the wide-sense stationary uncorrelated spreading (WSSUS) assumption is valid then the scattering function [L. J. Ziomek, Undenwater Acoustics: A Linear Systems Theory Approach (Academic, Orlando, FL, 1985)] completely defines the time and frequency characteristics of the channel. The scattering function is measured using traditional matched filter processing and the newer uncertainty product function (UPF) receiver [S. K. Mehta, Signal Design Issues for the Wigner Distribution Function and a New Twin Processor for the Measurement of Target andvor Channel Structures (Ph.D. dissertation, University of Rochester, Rochester, NY, 1991)]. The structure and performance of the UPF receiver will be discussed. The theory behind the scattering function and experimental results from sites off Halifax, Canada, and in Puget Sound will be presented.

\section{1:15}

2aUW11. Sound scattering at surface waves in the ocean, revisited. Oleg A. Godina) (NOAA/Atlantic Oceanographic and Meteorological Lab., 4301 Rickenbacker Cswy., Miami, FL 33149)

In the absence of air bubbles, sound scattering at the ocean surface is usually considered to result from surface roughness in a steady homogeneous fluid. However, the roughness is a manifestation of surface waves and is inevitably accompanied by fluid motion in a subsurface layer. Being time and space dependent, these currents provide an additional physical mechanism of sound scattering. Although negligible in most cases because of the smallness of surface-wave frequency compared to sound frequency, scattering due to currents is shown to be significant at near-specular directions. The difference between results of the quasisteady (frozen medium) approximation and that of rigorous theory is pronounced in the latter case. A visual interpretation of the volume scattering enhancement in nearspecular directions and of failure of the quasisteady approximation to predict it is given. The effect of this additional scattering mechanism on the frequency spectrum of sound scattered at the ocean surface in deep and shallow water is analyzed. [Work supported by NRC.] ${ }^{\text {a) }}$ On leave from $P$. P. Shirshov Oceanography Institute, Moscow, Russia. 
2aUW12. Broadband frequency dispersion prediction using shallow-water data from Tanner Bank. J. Paquin Fabre and James $\mathrm{H}$. Wilson (Neptune Sciences, Inc., 150 Cleveland Ave., Slidell, LA 70458)

Recent analyses of explosive charge reverberation and echo data [Davies et al. (1994)] showed a useful active broadband dispersion phenomena that was observed empirically in measured data for propagation paths within a mixed layer (ML). This Wilson Dispersion Phenomena (WDP) was originally noted in an environment which was characterized by a mixed layer. In April, 1994 an experiment [Holland et al. (1994)] was conducted under Navy sponsorship off the coast of California (Tanner Bank) in order to examine low-frequency active acoustic sources for use in characterizing bottom parameters in shallow water. This data set provided an ideal scenario for further examination of WDP. Upon preliminary examination of the Tanner Bank data set, the WDP was found to not only exist, but also to be predominant in environmental scenarios other than in the presence of a mixed layer. The phenomena proves to be highly dependent upon source-receiver geometry and environmental acoustic (EVA) characteristics such as sound-speed structure, bathymetry, bottom sediments, etc. It has been shown using the Tanner Bank data set that the occurrence of this phenomena can be modeled to a reasonable degree of accuracy using a time domain finite element parabolic equation model and a normal mode model. [Work supported by ONR.]
2aUW13. Method of determining the effective scattering cross section. Alexander E. Ekimov and Andrey V. Lebedev (Hydroacoustic Div., Inst. Appl. Phys., Russian Acad. Sci., 46 Ulyanov str., Nizhny Novgorod 603600, Russia)

This paper considers the method of determining the effective acoustical scattering cross section ( $S_{\text {eff }}$ ) for an object of arbitrary form or determining the contribution of its part into $S_{\text {eff }}$. The method is based on the determination of transfer coefficients by direct and reciprocal methods and calculation of $S_{\text {eff }}$ using the equation (without the diagram coefficient): $S_{\text {eff }}(\Omega) / S \sim\left\langle\mathbf{K}_{\text {rec }}^{2}(\Omega)\right\rangle_{F} \times\left\langle\mathbf{K}_{\text {dir }}^{2}(\Omega)\right\rangle_{V}$, where $S$ is the total square of the object, $\Omega$ is the angular position of the point in the external field, $\left\langle\mathbf{K}_{\mathrm{rec}}^{2}(\Omega)\right\rangle_{F}$ is the root-mean-square value of the transfer coefficient linking the force $F$ with the acoustic field, which is determined by the reciprocal method, $\left\langle\mathbf{K}_{\mathrm{dir}}^{2}(\Omega)\right\rangle_{V}$ is the root-mean-square value of the transfer coefficient linking the vibrovelocity $V$ on the surface of the object with the acoustic field, which is determined by the direct method. The root-meansquare values of $\mathbf{K}$ are determined over the necessary number of points on the surface of the object. The method proposed shows high noise protection in the experimental investigations. This method allows one to use a powerful generator and vibrator to increase the signal/noise ratio. The experimental results are presented.

Session 2pAAa

\title{
Architectural Acoustics: The Technical Committee on Architectural Acoustics Vern O. Knudsen Lecture
}

\author{
Leo L. Beranek, Chair \\ 975 Memorial Drive, Suite 804, Cambridge Massachusetts 02138-5755
}

Chair's Introduction-1:30

Invited Papers

$1: 35$

2pAAa1. Innovation in acoustical design. A. H. Marshall (Acoust. Res. Ctr., Univ. of Auckland, Private Bag 92019, Auckland, New Zealand)

Research clearly has a relationship to the design of concert halls, as does innovation. How do these merge in the owner's and user's best interests? In some cases the research precedes the design. In others the design itself becomes a research hypothesis for exploration, sometimes over many years. The noise in the system is political power. Aspects of the design process for several innovative halls will be discussed, together with the research background, predictive techniques applicable to each, and a summary of results achieved. 


\title{
Session 2pAAb
}

\author{
Architectural Acoustics: General \\ Dana S. Hougland, Chair \\ David L. Adams Associates, Inc., 1701 Boulder Street, Denver, Colorado 80211 \\ Chair's Introduction-3:15 \\ Contributed Papers
}

3:20

2pAAb1. New results for the music critic listening experiment at Dallas' Meyerson Symphony Center. David Lubman (David Lubman \& Assoc., 14301 Middletown Lane, Westminster, CA 92683) and Gary W. Siebein (Univ. of Florida, Gainsville, FL 32611)

A pilot experiment was conducted to compare the assessments of the acoustics of a concert hall made by music critics, musicians, and "others." The experiment, a cooperative effort between the ASA and the Music Critics Association of North America (MCANA), was performed in Dallas, TX at the McDermott concert hall of the Meyerson Symphony Center during MCANA's June 1994 meeting. The critics' meeting provided a unique opportunity to obtain acoustical assessments from a statistically significant number of critics attending concerts in the same hall. Standard survey forms and instructions were placed in the registration packets of about 75 music critics at check-in, and about 1000 noncritic volunteers attending concerts on two consecutive nights. The initial, disappointingly small number of survey forms returned by the critics was augmented by the critics' responses to a supplemental mailed appeal. Ultimately, 18 critics, nine musicians, and 20 "others" submitted survey forms for the Dallas Symphony concert. thirteen critics, 36 musicians, and 106 "others" submitted survey forms for the Houston Symphony concert held on the next night. With these new responses, statistically interesting, significant results can now be reported. [Work supported with private donor and TCAA Technical Initiative funds.]
3:35

2pAAb2. New developments of the "sound particle" concept in room acoustics. Domenico Stanzial (Natl. Res. Council of Italy, Cemoter Acoust. Dept., via Canal Bianco, 28, I-44044 Cassana, Ferrara, Italy) and Nicola Prodi (CIARM c/o Cemoter, Ferrara, Italy)

The concept of a sound particle, which is a small amount of acoustic energy that is supposed to move inside the sound field, has been used for a long time in room acoustics, principally in connection with the statistical interpretation of the reverberation theory and more recently with the raytracing-based computer simulations. New research on energetic properties of acoustic fields [G. Schiffrer and D. Stanzial, "Energetic Properties of Acoustic Fields," J. Acoust. Soc. Am. 96, 3645-3653 (1994)] leads to the possibility of rigorously defining, by means of the energy transfer velocity, the trajectories of sound particles. Furthermore, the general decomposition of the air-particle velocity $\mathbf{v}$ into one part $\mathbf{v}_{p}$, which has the same time dependence as the acoustic pressure $p$, and the other one $\mathbf{v}_{q}=\mathbf{v}-\mathbf{v}_{p}$ such that $\left\langle p v_{q}\right\rangle=0$, allows one to define two separate kinds of sound particle trajectories. These different behaviors are connected, respectively, to the instantaneous active and reactive acoustic intensities. A brief summary of the theoretical framework and some visualizations of sound particle trajectories for simple model fields will be presented. 


\title{
Session 2pAO
}

\section{Acoustical Oceanography: Acoustic Inversion of Fish and Plankton Ensembles II}

\author{
Christopher Feuillade, Cochair \\ Naval Research Laboratory, Stennis Space Center, Mississippi 39529-5004
}

Timothy K. Stanton, Cochair

Woods Hole Oceanographic Institution, Department of Applied Ocean Physics and Engineering, Bigelow 201, Woods Hole, Massachusetts 02543

Chair's Introduction-1:25

Invited Papers

1:30

2pAO1. Pulse modulation and frequency effects on long-range sonar displays of fish. David E. Weston (White Laird, 77 Wyke Rd., Weymouth, Dorset DT4 9QN, England)

Early work on long-range sonar displays of fish is reviewed, for observations in the Perranporth Sea area. There is a concentration on the complications in interpretation arising from the use of a variety of pulse types and carrier frequencies. A useful technique was the interleaved transmission of different pulses or frequencies, permitting a comparison of virtually synoptic records. For linear frequency-modulation pulses there are dramatic changes in display appearance when pulse duration or pulse bandwidth are changed, which must be allowed for in the inversion giving fish distribution and behavior. There are further differences and problems with noise-modulation pulses; although these can perform well, they do often give very poor pictures. In contrast to the pulse-type effects there is a relatively small dependence of detailed appearance on carrier frequency for frequencies 1,2 , and $3 \mathrm{kHz}$; reflecting the slow dependence of fish school target strength on frequency. Of course system performance does depend on frequency as it does on the whole system engineering. In spite of the complications it is possible to find useful information on fish numbers, school numbers, movement, target depth, and behavior generally.

\section{$1: 55$}

2pAO2. A theoretical and experimental basis for the measurement of fish migration using high-frequency acoustical scintillation. David M. Farmer, Mark Trevorrow, and Zhen Ye (Inst. of Ocean Sciences, 9860 W. Saanich Rd., Sidney, BC V8L 4B2, Canada)

Salmon migrating up the Fraser River produce an identifiable scintillation signal as they pass through high-frequency acoustic paths directed transversely across the flow. The scintillation field due to the fish can be identified by its differential motion with respect to that due to background turbulence which moves downstream with the river flow. The potential of this technique is explored in terms of a model for forward acoustic scattering from fish and the application of spatial aperture filtering and reciprocal transmission analysis. Results of recent laboratory and field studies will be presented.

2pAO3. Small scale spatial structure in zooplankton from high-frequency acoustical measurements. D. V. Holliday (Tracor Appl. Sciences, Anal. and Appl. Res. Div., 9150 Chesapeake Dr., San Diego, CA 92123)

Volume backscattering was measured at multiple discrete depths at frequencies of 0.165 and $1.1 \mathrm{MHz}$ from a mooring on the shelf-slope break at the northern edge of the San Pedro Basin near Los Angeles, CA. Attempts to understand temporal variability and vertical structure in the data from the mooring led us to make additional measurements along an onshore-offshore transect at this location at frequencies of $0.265,1.1,1.8$, and $3.0 \mathrm{MHz}$. These measurements, when processed with an inverse algorithm to determine plankton size and abundance, revealed both an onshore-offshore trend and small scale structure in this environment. Results of sampling the same environment with a multiple opening-closing net sensor system (MOCNESS) are consistent with the acoustical measurements, but at the same time highlight the difficulty of sampling a heterogeneous spatial distribution with a net system. [Work supported by ONR, NSF, and Tracor Applied Sciences.]

\section{2:45}

2pA04. Using broadband measurements and modeling near swimbladder resonance frequencies to determine biological characteristics of fish populations. Richard H. Love and Charles H. Thompson (Naval Res. Lab., Stennis Space Center, MS 39529-5004)

The Naval Research Laboratory (NRL) has been investigating low- to mid-frequency scattering from dispersed layers of fish for a number of years. Since 1988, NRL has conducted eleven experiments in a variety of regions, over shelf, slope, and deep waters. Scattering measurements have been made on many different fishes, from large commercially important fish, such as salmon, to small 
mesopelagic fishes that comprise the deep scattering layer. The frequency range of interest in these measurements, approximately 0.5 to $10 \mathrm{kHz}$, is the frequency range in which the swimbladders of many fish resonate. By employing a swimbladder scattering model in conjunction with the scattering data, information of the biological characteristics of the scatterers can be derived. This technique can provide information on the geographical distribution, depth, size, abundance, and swimbladder characteristics of the scatterers. The technique has been used successfully to provide detailed information on well-known species as well as to identify significant populations of fish in habitats where they were not expected. Examples of results for both well-known and unknown fish from both deep and shallow water are presented.

\title{
3:10-3:25 Break
}

\section{Contributed Papers}

\section{$3: 25$}

2pAO5. Determination of fish school structure from low-frequency backscattering. Redwood W. Nero and Christopher Feuillade (Naval Res. Lab., Stennis Space Center, MS 39529-5004)

A scattering model for predicting the backscattering from small ensembles of fish is used to determine the size and spatial arrangement of fish in schools. The model was initially developed to predict the low-frequency spectral signature of a fish school based on information on fish size and spacing. The inverse problem is to determine the size and spatial arrangement of fish within schools from measured scattering spectra. The method is applied to simulated data and measurements. Simulations provide information on the range of parameters that can be included in the inverse solution. Measurements are from several sources. They include examples of published data and recent measurements made by the Naval Research Laboratory. Results of this study suggest that low-frequency backscattering from fish schools may contain enough information to aid in their acoustic classification.

$3: 40$

2pA06. Sensitivity of the inverse problem to size-class selection. J. Michael Jech (Great Lakes Ctr., Buffalo State College, 1300 Elmwood Ave., Buffalo, NY 14222-1095), John K. Home (Buffalo State College, Buffalo, NY 14222-1095), and Denise M. Schael (Univ. of Zululand, KwaZulu/Natal, South Africa)

Fisheries sonar systems typically operate at discrete frequencies between 38 and $420 \mathrm{kHz}$. Can length-frequency distributions of aggregated fish be accurately estimated using available frequencies and the inverse problem? The inverse problem requires measured fish lengths and realistic scattering models. The size distribution of threadfin shad (Dorosoma petenense) in Lake Nornman was estimated using 120-, 200-, and 420-kHz data, and a recently developed scattering model [Jech et al., J. Acoust. Soc. Am. (in press 1995)]. Size distribution estimates were compared to length frequency measurements from purse seine catches. Fits of probability density functions (PDF's) using the inversion technique to those using length frequency measures were sensitive to the choice of fish size classes. Preliminary results indicate that estimation of length frequencies using multifrequency data and the inverse problem appears dependent on the shape of measured length-frequency distributions. [Work supported by NOAA Coastal Ocean Program (NA16RGO 492-01) and NSF (OCE-9115740).]

\section{3:55-4:00 Break}

\section{PANEL DISCUSSION:}

Panel Moderator: D. Vance Holliday

Panel Members: Stephen B. Brandt, Clarence S. Clay, David M. Farmer, Richard H. Love, Timothy K. Stanton, David E. Weston, Peter H. Wiebe

Session 2pEA

\section{Engineering Acoustics: Transducer Array Interactions and Underwater Transducers}

\author{
Roger T. Richards, Chair \\ Naval Undersea Warfare Center, Code 2131, New London, Connecticut 06320
}

Chair's Introduction-12:15

Invited Papers

12:20

2pEA1. High-power electromagnetic transducer array for Project Artemis. Donald P. Massa (Massa Products Corp., 280 Lincoln St., Hingham, MA 02043)

The low-frequency, high-power transducer array installed on the Mission Capistrano for use in Project Artemis was developed by Massa during the late 1950s and manufactured during 1961. The 33- $\times 50-\mathrm{ft}$ array contained 1440 Massa model TR-11C transducer elements. This paper will present a historical review of this $300000-\mathrm{lb}$ megawatt transducer array that successfully operated in the 
420- $\mathrm{Hz}$ region. The design of the transducer will be described, along with a few of the manufacturing procedures used to hold the resonance of the $180-1 b$ vibrating structure to within $10 \mathrm{~Hz}$. The 10 -mile-long receiving array, consisting of 200 towers $80 \mathrm{ft}$ tall containing hydrophones and lag lines for beam steering, will also be discussed.

\section{2:45}

2pEA2. Historic overview of the concept of velocity control relative to acoustic interactions in sonar arrays. David $L$. Carson (480 Rosecrans S1., San Diego, CA 92106)

This paper discusses the following historic points concerning the concept of velocity control for sonar projector arrays which have strong acoustic interactions among the transducer elements of a given array. (1) Diagnosis of problems that led to the introduction of the concept of velocity control and of including velocity control provisions in the design of the subject sonar projector arrays. (2) Some early observations concerning potential methods to provide velocity control and the corresponding early state of the art for a practical hardware realization of each of these potential methods. (3) An array design approach which specifically included velocity control provisions. (4) A general definition of velocity control in the context of the array design approach of item (3) above. (5) The first specific velocity control method used in the application of the concept of velocity control to the LORAD ARRAY. (6) Two more recent examples of implementation of specific velocity control methods applied to actual high-power, low-frequency, highly interactive projector arrays. (7) An example of the usefulness of inclusion of velocity control considerations in one's thinking concerning active sonar system design when interactive projector arrays are under consideration. (8) A parting thought-the discussion for the above seven points was in the context of analysis in the steady-state frequency domain; what possible relevance might these experiences have to present design problems concerned more with non-steady-state time domain analysis?

\section{1:10}

2pEA3. Approximating array mutual interactions. George W. Benthien (Naval Command, Control, and Ocean Surveillance Ctr., Res., Development, Test, and Evaluation Div., San Diego, CA 92152-5001)

In this paper some of the analytic and numerical techniques that have been applied to the array interaction problem will be reviewed. For problems where high accuracy is not required, many modelers have used an approximation for the mutual interactions that has come to be known as the Pritchard approximation. In this paper a new approximation technique will be presented which appears to be more accurate than the Pritchard approximation for the cases that have been examined. Results will be shown that compare this new approximation scheme both with the Pritchard approximation and with more accurate numerical computations. [Work supported by SPAWAR PMW-182.]

\section{$1: 35$}

2pEA4. Finite-element methods to analyze transducer array interaction. Richard E. Morrow (C+AES, Inc., 1223 Peoples Ave., Troy, NY 12180)

Understanding the effects of energy coupled from one transducer to an adjacent transducer in an array environment presents a challenging problem for the numerical analyst. Methods which minimize the number of equations to solve must be weighed against numerical accuracy and ease of model generation. Approaches based on finite elements, including "brute force" using planes of symmetry, the concept of marching frames, finite-element/boundary-element methods, and separation of the structural and acoustic solution domains for both linear and volumetric arrays are presented and evaluated. This evaluation will include a discussion on the adaptability of each method to parallel processing, especially using PVM software. Results for two array configurations of six MOD30 Class IV flextensional transducers will be presented for discussion.

\section{Contributed Papers}

2:00

2pEA5. An overview of variational methods. Harold C. Robinson (Naval Undersea Warfare Ctr., New London Detachment, New London, CT 06320)

Variational methods provide a powerful method for determining the behavior of complex physical systems without needing to solve the equations of state or satisfy the boundary conditions exactly. This technique has been widely applied in the areas of structural vibration and scattering, but has not been used much in the areas of transduction and array modeling. The advantage of this method over many others is that the answers obtained from variational principles are always significantly better than the inputs to them. A totally general method for deriving stationary variational principles from the equations of state and the boundary/initial conditions will be shown. Techiniques for choosing the inputs, or trial functions, judiciously will be discussed. The method will be illustrated using two examples: Determining the resonance frequencies of a clamped vibrating string and of a string with an attached point mass. The results of the variational treatment will be compared with exact analytical solutions of the problem for both accuracy and simplicity of solution. A brief historical overview of variational methods in acoustics will also be given.

\section{2:15}

2pEA6. Variation model for the radiation impedance of Class IV transducers III. Harold C. Robinson (Naval Undersea Warfare Ctr., New London Detachment, New London, CT 06320)

Previously [H. C. Robinson and E. A. McLaughlin, J. Acoust. Soc. Am. 97, 3300(A) (1995)], the modal radiation impedance for a pair of Class IV transducers was calculated using variational methods. This model determines distinct self and mutual radiation impedance contributions between pairs of surface velocity modes, including the effects of element orientation, without requiring exact solutions to the equations of motion and boundary conditions. However, in order to model the total radiation impedance of the transducers, suitable methods for combining these modal impedances must be determined, which in turn requires that some estimate of the relative importance of each modal contribution be made. One method of determining the relative strengths is singular value decomposition [G. W. Benthien, Naval Ocean Systems Center Technical Report 1329, Novernber 1989], which effectively allows one to determine the fluidloaded resonances of the transducer and their associated eigenvectors. The relative magnitudes of these in-water modes will determine their importance in the total impedance. The results of this combination will be incorporated in a modified equivalent circuit which includes the effects of 
the "banana" mode. Calculations using this circuit will be made to existing equivalent circuit models as well as to experimental data.

\section{2:30}

2pEA7. Close-packed acoustic array element interaction. John B. Blottman III (Naval Undersea Warfare Ctr., New London Detachment, New London, CT 06320) and Jean-Noël Decarpigny (Inst. Supérieur d'Electron. du Nord, 59046 Lille Cedex, France)

The acoustic array element interaction is an essential parameter determining transducer array behavior. It is characterized by the mutual radiation impedance. Low-frequency transducers in a volumetric array with small size constraints are subject to much larger interaction and scattering than in conventional arrays. This paper provides an overview of an investigation of the modal components of mutual radiation impedance. The in vacuo eigenmodes of a single element are determined using the ATILA finite-element method. Selection of the modal expansion terms will be based on their radiation efficiencies. Modal radiation impedances are generated using the EQI boundary integral equation method. A simple multimode equivalent circuit model of the in situ projectors, including the interaction effects, is proposed. Comparison of the results to a full finiteelement model of an array is presented.

\section{2:45-3:00 Break}

2pEA8. Sensor-actuator interactions in the NRL-ABC platform. Robert D. Corsaro and Brian Houston (Naval Res. Lab., Code 7130, Washington, DC 20375-5350)

The ABC research platform consists of a large array of multifunctional transducers mounted on a backing structure. It was specifically constructed for underwater studies of sensor/actuator coupling mechanisms. The platform transducers are represented as a 15 tile array of $\mathrm{ABC}$ tiles, where each tile contains a large area actuator, pressure sensor, and velocity sensor, where the latter is constructed by summing and integrating the outputs of four accelerometers. Acoustic characteristics of the ABC tiles and platform were evaluated in the NRL Large Pool Facility, both in the freefield and when mounted on a backing structure. This paper presents results and analysis of inter- and intra-tile coupling mechanisms, including particularly an evaluation of the role of the backing structure.

\section{$3: 15$}

2pEA9. Acoustic pressure field evaluation of an annular cylindrical array for underwater pipeline inspection. R. Sumangala and $P$. R. Saseendran Pillai (Dept. of Electron., Cochin Univ. of Sci. and Technol., Cochin-682 022, India)

Evaluation of the effective acoustic pressure field of a continuous wave, optimum spaced, point source, annular cylindrical array for underwater pipeline inspection is featured in this paper. A section of the array comprised of $m \times n$ elements is selectively energized to illuminate the region to be tested and a similarly grouped set of remaining array elements function as receivers. A steady half-power beamwidth and sidelobe levels were obtained for a radius of the array greater than $40 \lambda$. The portion of the energy reflected or reradiated from the contours of the pipeline and captured by the point source elements being nominally small can be neglected for all practical purposes. Effective acoustic pressure is computed as the Fourier transform of the impulse response of the array elements $h_{m n}(r, t)$ determined using the Green's function approach. The total effective acoustic pressure over the contour of the pipeline is the sum of the weighted contributions from all radiating set of elements. A good focusing effect can be achieved by optimizing the number of elements and the radius of the array. [Work supported by CSIR INDIA.]
2pEA10. Transmission and reception performance of multilayered transducer structures. David J. Powell (Dept. of Ocean Eng., Florida Atlantic Univ., Boca Raton, FL 33431), Robert Y. Ting (Naval Undersea Warfare Ctr., Orlando, FL 32856-8337), and Thomas R. Howarth (Naval Res. Labs., Washington, DC 20375)

Multilayered transducer structures offer the potential for greater performance in terms of increased radiated acoustic power and improved reception characteristics. In earlier work, a unidimensional modeling approach was presented, that was shown to provide a means of accurately predicting the in-air electrical impedance characteristics of a range of different laminated transducer structures [J. Acoust. Soc. Am. 97, 3299(A) (1995)]. These prototype devices have since been encapsulated within polyurethane rubber ready for in-water acoustic testing. This paper presents a theoretical and experimental analysis of both the transmission and reception performance characteristics of this group of multilayered devices. The effects of intermediate bondlines and electrode layers will be considered in terms of changes to both the device's sensitivity and its resonant frequency. Transducer performance will be assessed via the standard figures of merit, TVR and FFVS, in conjunction with its pulse-echo transient response. Theoretical predictions from the unidimensional model are in good agreement with experimentally obtained values. The polyurethane encapsulant was found to have detrimental effects on overall transducer performance. Beam profiles for the laminated devices have been recorded experimentally and are compared to the responses of their singlelayer counterparts. [Work sponsored by the Office of Naval Research.]

\section{3:45}

2pEA11. A reinforced Neoprene rubber boot for the barrel-stave flextensional projector. Dennis F. Jones (Defence Res. Establishment Atlantic, P.O. Box 1012, Dartmouth, NS B2Y 3Z7, Canada)

The Class I barrel-stave flextensional projector is a lightweight and compact underwater sound source that is well-suited to low-frequency sonar and oceanographic applications. By modifying a few of the parts used in the Class I projector, a high-power Class II or broadband Class III barrel-stave projector can be constructed, which is testimony to the versatility of the basic barrel-stave design. These projectors require gaps, between adjacent staves, that are sufficiently wide for free-stave vibration at the operating drive levels and water depths. Gap widths of about $1 \mathrm{~mm}$ are typical. A rubber boot is stretched over the projector to inhibit the ingress of seawater through the gaps. However, since the gap widths and boot wall thicknesses are similar, the boots can be forced into the gaps by hydrostatic pressure, causing significant variations in the projector performance parameters with water depth [D. F. Jones and M. B. Moffett, J. Acoust. Soc. Am. 93, 2305(A) (1993)]. To minimize these variations, a new rubber boot with reinforcements in the vicinity of the gaps has been fabricated and tested on a Class II barrel-stave projector. Measured results showing performance stability with depth are presented.

\section{4:00}

2pEA12. Assessment of a fractal model for corona discharges in salt water. H. M. Jones, J. C. Epsinosa, M. L. Galloway, A. M. Gleeson, and R. L. Rogers (Appl. Res. Labs., Univ. of Texas, P.O. Box 8029, Austin, TX 78713-8029)

The prebreakdown corona phase of a spark discharge in salt water produces a measurable acoustic pulse. This pulse is produced by the formation and collapse of a vapor bubble. The plasma has a multiply fingered shape, and it creates a bubble with a corresponding acoustic pulse. The rate of formation of plasma fingers may be a fractal dimension of the size of the discharge. A fractal model of the growth of the corona discharge can be used to connect the variables of voltage, current, and bubble wall acceleration. The dependence of the acoustic and electrical response and thus the fractal dimension of the corona growth on liquid conductivity, and applied voltage are investigated. The acoustic data are used to infer the bubble wall acceleration while the current-voltage data are measured directly and used to determine the total resistance of the plasma. The corona is formed by the application of high-amplitude electric fields to a set of electrodes that are immersed in the liquid. [Work supported by the Office of Naval Research under Grant No. N00014-94-1-0150.] 


\title{
Session 2pPA
}

\section{Physical Acoustics: Nonlinear Acoustics of Rocks II}

\author{
Andrew N. Norris, Chair \\ Department of Mechanics and Aerospace Engineering, Rutgers University, P.O. Box 909, Piscataway, \\ New Jersey 08855-0909
}

Chair's Introduction-1:30

Invited Papers

1:35

2pPA1. Manifestation of nonlinear elasticity in rock: Convincing evidence over large frequency and strain intervals from laboratory studies. Paul A. Johnson (EES-4, MS D443, Los Alamos Natl. Lab., Los Alamos, NM 87545 and Université Pierre et Marie Curie, Bureau des Mécaniques, Tour 22, 4, Pl. Jussieu, 75252 Paris Cedex 05, France) and Patrick N. J. Rasolofosaon (Institut Français du Pétrole, Rueil Malmaison Cedex, France)

Nonlinear elastic response in rock is established as a robust and representative characteristic of rock rather than a curiosity. This behavior is illustrated from a variety of experiments conducted over many orders of magnitude in strain and frequency. The evidence leads to a pattern of unifying behavior in rock: (1) Nonlinear response in rock is enormous; (2) the response takes place over a large frequency interval (dc-10 $\mathrm{Hz}$ at least); (3) the response not only occurs, as is commonly appreciated, at large strains but also at small strains where nonlinear response and the manifestations of this behavior are commonly disregarded. Nonlinear response may manifest itself in a variety of manners, including a nonlinear stress - strain relation (hysteretic/discrete memory), nonlinear dissipation, harmonic generation, and resonant peak shift, all of which are related. The experiments described include: quasistatic stress - strain tests (strains of $10^{-4}-10^{-1}$ at frequencies near dc- $1 \mathrm{~Hz}$ ); torsional oscillator experiments (strains of $10^{-4}-10^{-7}$, frequencies between 0.1 and $100 \mathrm{~Hz}$ ); resonant bar experiments (strains of $10^{-4}-10^{-8}$, frequencies between $10^{3}$ and $10^{4} \mathrm{~Hz}$ ); and dynamic, propagating wave experiments (strains of $10^{-6}-10^{-9}$, frequencies between $10^{3}$ and $10^{6} \mathrm{~Hz}$ ). [Work supported by OBES/DOE through the University of California and the Institut Français du Pétrole.]

2pPA2. Hysteresis in elastic behavior: The connection between low-frequency response and acoustic properties of rocks. Katherine R. McCall (Earth and Environ. Sciences Div., Los Alamos Natl. Lab., Los Alamos, NM 87545), Robert A. Guyer (Univ. of Massachusetts, Amherst, MA 01003), and Lei Zhu (New Mexico State Univ., Las Cruces, NM 88003)

The strain response of rock to quasistatic stress cycles (e.g., $10^{-3} \mathrm{~Hz}$ ) is highly nonlinear, hysteretic, and displays diserete memory. Rocks also display unusual nonlinear behavior in acoustic wave experiments (e.g., $10^{4} \mathrm{~Hz}$ ). Nonlinearity and hysteresis are prominent features in elastic measurements on rocks. This observation is the key to making the connection between low-frequency (quasistatic) and high-frequency (acoustic) measurements, e.g., between static modulus and dynamic modulus. A new paradigm has been developed for the description of the elastic behavior of rocks and other consolidated materials. This paradigm uses the statistical properties of an ensemble of micron-scale hysteretic mechanical units to describe the elastic response of a macroscopic piece of material. It provides a recipe for inverting stress-strain data (low-frequency data) for the distribution of hysteretic mechanical units. From this distribution, the high-frequency acoustic response of the macroscopic piece of material can be predicted. The new paradigm will be described in principle and in application. Quasistatic stress-strain data on sandstone lead to predictions for dynamic modulus and resonant response that agree well with experiment.

2pPA3. Shear hysteresis of rock at intermediate strains. Brian P. Bonner, Chantel Aracne-Ruddle, and Cindy Shew (Earth Sciences Div., Lawrence Livermore Natl. Lab., Livermore, CA 94551)

Rocks with appropriate microsetructure are nonlinear with hysteresis in the strain range 10-6 to 10-3 while showing little permanent damage during deformation. Nonlinear effects alter strong motions produced by earthquakes and explosions and may lead to new methods for relating the mechanical properties of rock to microstructure and transport properties. Results of laboratory torsion experiments will be presented to demonstrate that various nonlinear responses appear when granite and sandstone samples are driven in harmonic motion at low frequencies with increasing amplitudes. Modulus reduction, amplitude-dependent attenuation and harmonic 
generation occur and depend on microstructural parameters, including microfracture density and pore fluid content. Friction and adhesion at internal surfaces and the deformation of fluid phases in pores all play a role in these observed phenomena. Numerical constitutive models which include micromechanics are being developed to simulate nonlinear behavior for comparison with experiment. [Sponsored by OBES geosciences and performed under the auspices of the U. S. DOE by LLNLL under Contract W-7405ENG-48.]

\section{2:40}

2pPA4. Thermodynamical aspects of modeling the mechanical response of granular materials. David Elata (Earth Sci. Div., Lawrence Livermore Natl. Lab., Livermore, CA 94551)

In many applications in rock physics, the material is treated as a continuum. By supplementing the related conservation laws with constitutive equations such as stress-strain relations, a well-posed problem can be formulated and solved. The stress-strain relations may be based on a combination of experimental data and a phenomenological or micromechanical model. If the model is physically sound and its parameters have a physical meaning, it can serve to predict the stress response of the material to unmeasured deformations, predict the stress response of other materials, and perhaps predict other categories of the mechanical response such as failure, permeability, and conductivity. However, it is essential that the model be consistent with all conservation laws and consistent with the second law of thermodynamics. Specifically, some models of the mechanical response of granular materials proposed in literature, are based on intergranular contact force-displacement laws that violate the second law of thermodynamics by permitting energy generation at no cost. This diminishes the usefuiness of these models as it invalidates their predictive capabilities. [This work was performed under the auspices of the U.S. DOE by Lawrence Livermore National Laboratory under Contract No. W-7405-ENG-48.]

\section{3:10-3:15 Break}

\section{3:15}

2pPA5. Theoretical modeling of nonlinear surface waves. M. F. Hamilton, Yu. A. Il'inskii, and E. A. Zabolotskaya (Dept. of Mech. Eng., Univ. of Texas, Austin, TX 78712-1063)

Nonlinear effects in surface waves, like those in bulk elastic waves, are enhanced dramatically by microinhomogeneous features such as cracks and grains that are common in rocks. Since surface waves experience less geometrical spreading loss than bulk waves, nonlinearity can be even more pronounced than in bulk waves. A brief review of theoretical models for studying nonlinear surface wave propagation will be presented. The models are based on the theory developed by Zabolotskaya [J. Acoust. Soc. Am. 91, 2569-2575 (1992)] for nonlinear Rayleigh waves in isotropic solids. In subsequent articles published in the Journal the theory was used to study harmonic generation, waveform distortion, and shock formation in plane waves, cylindrical waves, and diffracting surface wave beams. Radiation from both time harmonic and pulsed sources was investigated. Reported values for second- and third-order elastic moduli were used to calculate coefficients of nonlinearity for a number of rocklike materials. The theoretical model was recently extended to encompass nonlinear Stoneley, Scholte, and Lamb waves, and to include effects of anisotropy and piezoelectricity. Most experiments reported on nonlinear surface waves are associated with the development of nonlinear SAW devices in the 1970s. Several of these experiments will be revisited, and new interpretations of the measurements will be offered. [Work supported by NSF, the Office of Naval Research, and the Schlumberger Foundation.]

\section{Contributed Papers}

$3: 35$

2pPA6. Reflections on the nonlinear equation of state in rock based on experiment. James A. TenCate (EES-4 MS D443, Los Alamos Natl. Lab., Los Alamos, NM 87545)

Measurements were made of the propagation of I-D nonlinear waves (i.e., Young's mode) in a bar of Berea sandstone $3.8 \mathrm{~cm}$ in diameter and 1.8 $\mathrm{m}$ long. Both waveforms (time domain) and spectra (frequency domain) were measured. The experimental results were then compared with waveforms calculated from a numerical scheme based on the simple wave solution for 1-D waves in rock using a classical nonlinear equation of state. The numerical solution is written in MATLAB and runs quickly on a small personal computer. Attenuation was added by propagating the waveform a small distance, transforming the waveform into the frequency domain, and applying the attenuation, and then transforming back into the time domain and propagating the new waveform. The same method was applied earlier for nonlinear propagation of a sound wave in a tube of air by Pestorius and Blackstock. The experiments and simulations clearly demonstrate that a classical nonlinear equation of state is incomplete or inappropriate for describing or modeling nonlinear propagation in sandstone. Results from another model (Van Den Abeele, paper 2aPA8) suggest the same conclusions. [Work supported by OBES/DOE through the University of California.]
3:50

2pPA7. Experimental determination of the linear and nonlinear dynamic moduli of rock from quasistatic measurements. L. Zhu (Phys. Dept., New Mexico State Univ., Las Cruces, NM 88003), R. A. Guyer (Univ. of Massachusetts, Amherst, MA 01003), K. R. McCall (Los Alamos Natl. Lab., Los Alamos, NM 87545), G. N. Boitnott (New England Res., Inc., White River Junction, VT 05001), L. B. Hilbert, Jr. (Univ. of Califormia, Berkeley, CA 94720), and T. J. Plona (Schlumberger-Doll Res., Ridgefield, CT 06877)

The central construct of a new theory of the elastic behavior of consolidated materials is the density in Preisach-Mayergoyz (PM) space. PM space is an abstract space in which the response of the mechanical units in the material to changes in stress state can be tracked. The theory provides a recipe for using quasistatic data to determine $\rho_{\mathrm{PM}}$, the density of mechanical units in PM space. This recipe has been applied to quasistatic stress/strain data on three sandstones samples: (a) Berea I, (b) Berea II, and (c) Castlegate. The density of mechanical units $\rho_{\mathrm{PM}}$ was found for each sample. From $\rho_{\mathrm{PM}}$ the dynamic behavior of the samples can be predicted. Using the experimentally determined $\rho_{\mathrm{PM}}$ for each of the three samples the strain response to complicated stress protocols is predicted and the linear and nonlinear dynamic moduli of the samples are found as a function of pressure. The predictions agree well with experiments that test them. 
nonlinearity. The model is based on the assumption that the stress applied to a single crack is the same as that applied to the whole body, that is valid for a low concentration of cracks. The crack behavior was described using the model in which a crack can be presented as an elastic contact of two rough surfaces, pressed one to another under the action of internal stresses in the surrounding solid. The nonlinear behavior of a crack is due to the contact area changing when the crack is undergoing stress or extension. Linear and nonlinear elastic constants of the medium with cracks are calculated; it is shown that in this medium negative values of Poisson's ratio and anomalously high values of the third-order constants are possible.

TUESDAY AFTERNOON, 28 NOVEMBER 1995

ST. LOUIS F, 1:30 TO 4:30 P.M.

\title{
Session 2pPP
}

\section{Psychological and Physiological Acoustics: Noise Discrimination, Binaural Processing and Masking}

\author{
William A. Yost, Chair \\ Parmly Hearing Institute, Loyola University of Chicago, 6525 North Sheridan Road, Chicago, Illinois 60626
}

Contributed Papers

1:30

2pPP1. Noise discriminability. I. A comparison of the effects of temporal position and bandwidth. Martin E. Rickert and Donald E. Robinson (Dept. of Psych., Indiana Univ., Bloomington, IN 47405)

In previously reported work [S. F. Coble and D. E. Robinson, J. Acoust. Soc. Am. 92, 2630-2635 (1992); M. E. Rickert and D. E. Robinson, J. Acoust. Soc. Am. 93, 2386(A) (1993)] listeners discriminated among trials consisting of eiether two identical samples of noise or two nonidentical samples. Nonidentical samples were generated by replacing a segment of noise presented during the first interval with a new segment. Although the long-term power spectrum of the segments was the same, the temporal position at which segments were replaced had a significant effect on discriminability: performance was best when changes occurred at the end and was poorest when changes occurred at the beginning. In the present study the effects of temporal position were measured under two spectral conditions. In one condition, noise samples were identically filtered $(100-3000 \mathrm{~Hz}$ or $455-655 \mathrm{~Hz})$ for the entire stimulus duration (50 $\mathrm{ms}$ ). The effect of temporal position is reduced with narrow-band stimuli but is not eliminated. In a second condition, the bandwidth was varied within each sample such that one segment was wideband $(100-3000 \mathrm{~Hz})$ and the other narrow band $(455-655 \mathrm{~Hz})$. Overall performance with mixed stimuli (1) is similar to that with pure wideband noise when the uncorrelated segment is wideband, and (2) is similar to that with pure narrow-band noise when the uncorrelated segment is narrow band. [Work supported by AFOSR.]

\section{$1: 45$}

2pPP2. Noise discriminability. II. Leaky integrator models. Donald E. Robinson and Martin E. Rickert (Dept. of Psych., Indiana Univ., Bloomington, IN 47405)

Several two-interval, same-different experiments involving the discriminability of samples of noise have been reported previously. A striking feature of these data is the effect of temporal position: samples that are altered near the end are more discriminable than those in which the change occurs earlier. This effect occurs over a wide range of durations and bandwidths. Here two models are reported that describe these results. In each model the waveforms from the two temporal intervals are jittered in amplitude, filtered, and squared or rectified. In one model, the resulting waveforms are passed through a leaky integrator and subtracted from one another. This difference waveform is squared and passed through a second leaky integrator. A sample of the output of the second-stage leaky integra- tor taken at the end of the integration period is used as a decision variable from which hit and false alarm rates are obtained and $d^{\prime}$ is computed. In the other model, the squared waveforms are multiplied by an exponential weighting function before subtraction. A signal-to-noise statistic is then used to obtain an estimate of $d^{\prime}$. The fitting parameters for each model are the variance of the internal noise process and the time constant of either the second-stage integrator or of the exponential weighting function. The results of simulations will be compared with data from two experiments, and the relation of these models to others involving leaky integrators will be discussed. [Work supported by AFOSR.]

\section{2:00}

2pPP3. Localization of a virtual acoustic target in the presence of a distractor. M. A. Stellmack and E. A. Macpherson (Waisman Ctr., Univ. of Wisconsin-Madison, 1500 Highland Ave., Madison, WI 53705)

Localization of a virtual acoustic source in isolation was compared to that for the same source played in the presence of a distractor source. The target stimulus was a tonal complex consisting of a $253-\mathrm{Hz}$ fundamental and all harmonics below $14 \mathrm{kHz}$. The distractor stimulus was a similar tonal complex with a $353-\mathrm{Hz}$ fundamental. Both target and distractor were $250 \mathrm{~ms}$ in duration. Virtual sources were generated by filtering the source stimuli with individualized head-related transfer functions (HRTF's), and the resulting stimuli were presented over headphones. Listeners were instructed to place an acoustic pointer (wideband noise source) at the same apparent position as the target by pressing buttons on a response terminal to manipulate the pointer position. Listeners were allowed to play the test stimulus and pointer at will during each trial. The variability of the pointer responses was similar in the two conditions (target alone and target-plusdistractor), but the difference between the actual target position and the average pointer position was larger when a distractor was present. Responses in the target-plus-distractor conditions tended to be biased toward the distractor position, particularly when the target and distractor were on the same side of the head.

\section{2:15}

2pPP4. The relationship between binaural fusion and binaural interference. Raymond H. Dye, Jr. (Parmly Hearing Inst., Loyola Univ., 6525 N. Sheridan Rd., Chicago, IL 60626)

A stimulus-classification paradigm was used to examine the extent to which judgments of the laterality of $753-\mathrm{Hz}$ targets were influenced by distractors of different frequency. On each trial, the target was presented with one of ten different interaural differences as was the distractor. Each 
test interval was preceded by a diotic presentation of the target alone. During a block of 100 trials, each combination of target-distractor interaural differences was presented once. The relative weights given to the target and the distractor were assessed by the slope of the best linear boundary between left and right responses. Data were collected for conditions in which the cues were interaural differences of level or time. For all conditions for which target and distractor weights were obtained, binaural interference was measured by determining threshold interaural differences for conditions in which the target was presented alone and in the presence of a diotic distractor. For most subjects, greater interference was obtained when the distractor was at a frequency that was given great weight, although the frequencies weighted most heavily for interaural differences of time were not the same as those weighted heavily for interaural differences of level. [Work supported by NIH.]

\section{$2: 30$}

2pPP5. Breakdown of echo suppression with multiple echoes. Sandra J. Guzman and William A. Yost (Parmly Hearing Inst., Loyola Uniy. Chicago, 6525 N. Sheridan Rd., Chicago, IL 60626)

If a sound is followed a few milliseconds later by its echo, the echo is often suppressed. If a train of repeating sources and echoes is established and then the source and echo are reversed, there is a temporary breakdown of echo suppression. A procedure has been developed for studying echo suppression in which listeners attend to the last sounds in a series of repeating sounds. Using this procedure the effect of multiple echoes (up to seven) on echo suppression and the breakdown of echo suppression was investigated. In all cases there is a breakdown in echo suppression when the echoes are changed in a number of ways. The results indicate an interaction among number of echoes, spatial location of the echoes, and the timing among the echoes in both echo suppression and its breakdown. The results will be discussed in terms of the concept that echo suppression depends on the listener's prior experience in the acoustic environment. [Work supported by NIDCD and AFOSR.]

\section{$2: 45$}

2pPP6. Temporal masking of amplitude modulation detection with a wideband noise carrier. Stanley Sheft and William A. Yost (Parmly Hearing Inst., Loyola Univ. Chicago, 6525 N. Sheridan Rd., Chicago, IL 60626)

Thresholds were measured for detecting amplitude modulation (AM) of a gated wideband noise signal that was either immediately preceded or followed by a modulated noise masker. The signal AM rate was $2.5,5,10$. or $20 \mathrm{~Hz}$ and the masker $A M$ rate ranged from 2.5 to $40 \mathrm{~Hz}$. Signal duration was 200 or $400 \mathrm{~ms}$ and masker duration was $400 \mathrm{~ms}$. The maximum elevation in signal AM detection thresholds due to the presence of masker modulation ranged across subjects from roughly 4 to $10 \mathrm{~dB}$ with the amount of masking generally greatest when the signal and masker AM rates were either close or the same. The extent of tuning in the modulation domain was broad in both the forward and backward masking conditions. When not modulated, the masker can be viewed as a fringe of the signal carrier. Separate conditions evaluated the effects of carrier fringes on signal AM detection. At low AM rates. the addition of either the forward or backward fringe benefitted AM detection. These results suggest that the decrement in low-rate AM detection due to carrier gating is not solely attributable to the neural onset response and short-term adaptation. [Work supported by NIH and NSF.]

\section{3:00-3:15 Break}

\section{$3: 15$}

2pPP7. Detection and temporal integration of brief band-limited increments and decrements within a broadband noise signal. $C$. Formby (Div. of Otolaryngol-HNS, Dept. of Surgery, Univ. of Maryland School of Medicine, 419 W. Redwood St., Baltimore, MD 21201), M. G. Heinz (Johns Hopkins Univ., Baltimore, MD 21218), S. E. Hargus, and J. W. Zeiders (Univ. of Maryland School of Medicine, Baltimore, MD 21201)

Detection thresholds were measured for brief band-limited increments and decrements within a broadband noise signal. The signal consisted of three temporal regions, where the first and third regions served as markers for the increment or decrement in the second region. The first and third regions had a flat magnitude spectrum from $0-6000 \mathrm{~Hz}$ and were presented at an average $N_{0}=40 \mathrm{~dB} / \mathrm{Hz}$. The second temporal region contained an incremented or decremented frequency band- or notch-width selected from $W_{N}=62$ to $6000 \mathrm{~Hz}$, with logarithmic center frequency $2500 \mathrm{~Hz}$ and spectrum level $S_{0}$. The temporal increment or decrement was centered within the 500-ms signal. For each $W_{N}$ condition, detection threshold was measured as a function of increment or decrement duration $\left(P_{N}=10\right.$ to $480 \mathrm{~ms}$ ) by tracking $S_{0}$ adaptively. Increments were detectable for all combinations of $W_{N}$ and $P_{N}$, but decrements were consistently detectable only for $W_{N}>500 \mathrm{~Hz}$. Increment and decrement thresholds varied inversely with $W_{N}$ and $P_{N}$, ranging from $13 \mathrm{~dB}$ for small increment $W_{N}$ and $\boldsymbol{P}_{N}$ to $1.5 \mathrm{~dB}$ for large $W_{N}$ and $\boldsymbol{P}_{N}$. Temporal integration functions were fitted to the increment detection results. The resulting estimates of integration time constants varied inversely with $W_{N}$ from 67 to $7 \mathrm{~ms}$. [Research supported by NIH.]

\section{3:30}

2pPP8. Comparison of peak and energy detection for auditory masking of tones by narrow-band noise. Julius L. Goldstein (Central Inst. for the Deaf, $818 \mathrm{~S}$. Euclid Ave., St. Louis, MO 63110)

Classical energy detection theory [Green and Swets, Signal Detection Theory and Psychophysics (Wiley, New York, 1966)] predicts that masking of tones by Gaussian noise is limited by the variability of detector responses and by internal auditory noise. It elegantly relates detection variability to masker duration-bandwidth product $(T W)$. The theory accounts reasonably for Gaussian maskers with fixed RMS levels, but fails when the level is randomly roved with each stimulus presentation, suggesting that the auditory system detects waveform cues. A solution to this problem is proposed in which energy detection is replaced with the dual cues of envelope peak detection and normalized envelope peak detection, which are optimally processed as in classical theory [Goldstein and Hall, J. Acoust. Soc. Am. 97, 3330(A) (1995)]. The model was studied with periodic noise maskers comprising successive harmonics having uniform amplitudes and random phases, and with Gaussian noise maskers. Fixed-level masked threshold predictions converge to $\sim-4 \mathrm{~dB}$ SNR for both noises when $T W>4$, with small differences otherwise. Roved-level predictions for the periodic noise masker (one period duration) are $\sim 5 \mathrm{~dB}$ higher. Agreement exists between model predictions and the systematic masking data of Richards [J. Acoust. Soc. Am. 91, 3424-3435 (1992)]

\section{3:45}

2pPP9. Optimal pattern recognition in signal-dependent noise as a possible basis for the Weber-Fechner laws. Nicholas C. Makris (Naval Res. Lab., Washington, DC 20375)

The apparent logarithmic response of human auditory and visual perception to intensity stimulus exhibited in the Weber-Fechner laws is interpreted from the perspective of optimal pattern recognition in signaldependent noise. The stochastic behavior of acoustic and optical fields received from both fluctuating sources and scatterers can often be well approximated with circular complex Gaussian random (CCGR) variables. Averaged intensity from a CCGR field has a standard deviation proportional to the mean. Therefore, intensity images derived from CCGR fields 
have signal-dependent noise known as speckle. Taking the logarithm of such intensity images homomorphically transforms the signal-dependent noise into additive signal-independent noise. It has recently been shown that matched filtering such images with hypothetical patterns in the logarithrnic domain provides an optimal method for pattern recognition according to the independent perspectives offered by minimum variance unbiased estimation with Fisher information, optimal filtering, and information theory [N. C. Makris, Opt. Lett. (to be published 1995)]. This provides a mathematical justification for the use of logarithmic measurements to efficiently convey information for pattern recognition, and in this context may also provide a basis for the apparent logarithmic response of human auditory and visual perception to intensity stimulus.

2pPP10. Durational effects on masked thresholds in noise as a function of signal frequency, bandwidth, and type. Jim J. Hant, Brian P. Strope, and Abeer A. Alwan (Dept. of Elec. Eng., UCLA, 405 Hilgard Ave., Los Angeles, CA 90095)

The reported perceptual experiments are aimed at quantifying the relationship between masked thresholds of signals within noise as a function of signal center frequency, duration, and signal type (pure tones or 1-8 critical-band noises). Tests are adaptive 2AFC with the signals' center frequencies ranging between 0.4 and $6 \mathrm{kHz}$ and their durations, between 10 and $300 \mathrm{~ms}$. The masker is flat noise with a spectrum level of $36 \mathrm{~dB}$ $\mathrm{SPL} / \mathrm{Hz}$ sampled at $16 \mathrm{kHz}$. Four subjects participated in the experiments. Critical-band theory accurately predicts the masked thresholds for tones at $300 \mathrm{~ms}$. Tone thresholds increase almost linearly with decreasing logarithmic duration, and these durational effects are more pronounced at low frequencies. Thresholds for $1 \mathrm{CB}$ noise and tone signals are similar at short durations (10-30 ms). For longer durations and for wideband noises, however, differences both in thresholds and the way the thresholds change with duration are observed. Possible explanations in terms of changing auditory filter bandwidths, temporal integration, temporal onset detection and/or an interplay between these factors will be presented. The data are used to predict the masked thresholds of stop bursts in the presence of noise. [Work supported by NIH.]

4:15

2pPP11. Modeling the effects of spectral density on the masking of additive noise by vowel-like sounds. B. Espinoza-Varas (Communication Sciences \& Disorders, Univ. Oklahoma Health Sciences Ctr., Oklahoma City, OK 73190) and Muralidhar R. Kudumala (Univ. of Oklahoma, Oklahoma City, OK 73190)

The masking of additive noise by vowel sounds is relevant to speech coding. Since the spectral density of vowels is relatively low, and varies inversely with fundamental frequency, it may limit the masking effectiveness of vowels. In this paper, an excitation-pattern (EP) approach (Moore and Glasberg, 1987) is used to model effects of spectral density on masking. EP predictions were obtained for masker densities ranging from 1.0 spectral component per $\mathrm{Hz}$ to 1.0 per $220 \mathrm{~Hz}$, and target densities ranging from 1.0 component per $\mathrm{Hz}$ to 1.0 per $220 \mathrm{~Hz}$. In all conditions, the overall power of masker and targets was 64.0 and $50.3 \mathrm{~dB}$, respectively. For each combination of masker and target density, the EPs of the masker alone $\left(\mathrm{EP}_{m}\right)$ and of the masker-plus-target $\left(\mathrm{EP}_{m+t}\right)$ were obtained. The EP difference, $\mathrm{EPD}=\left(\mathrm{EP}_{m+t}\right)-\left(\mathrm{EP}_{m}\right)$, at each ERB was integrated (across all ERBs) to obtain an index of target detectability. For masker densities lower than -1.0 component per $100 \mathrm{~Hz}$, detectability improved as masker density decreased; the effect of target density was small. Predictions are compared to detection thresholds for additive noise masked by either /i/ or /a/, with $100-$ or $200-\mathrm{Hz}$ fundamentals. [Work supported by OCASTHR4-064.]

\title{
Session 2pSA
}

\section{Structural Acoustics and Vibration: Wiener-Hopf Methods in Structural Acoustics}

\author{
Paul E. Barbone, Chair \\ Department of Aerospace and Mechanical Engineering, Boston University, 110 Cummington Street, Boston, \\ Massachusetts 02215
}

Invited Papers

2pSA1. Scattering from fluid-loaded junctions of plates and shells. Douglas A. Rebinsky and Andrew N. Norris (Dept. of Mech. and Aerospace Eng., Rutgers Univ., Piscataway, NJ 08855-0909)

This paper will review recent results for several related problems involving plate and shell junctions in the presence of fluid loading. First, a general solution will be discussed which gives the acoustic and structural scattered response for two joined flat plates under unilateral fluid loading. By combining this with a related solution for the admittance matrix of the fluid-loaded plates, the behavior of a pair of semi-infinite plates in contact with fluid on one side and a mechanical structure on the other can be modeled. For simplicity, the intemal frame is characterized by an impedance matrix. Based on these results, a perturbation solution can be developed for two joined curved shells under unilateral loading. The leading order term in the expansion is the previously solved case of two joined flat plates, and the next term gives an approximation of the diffracted longitudinal and shear wave fields originating from the junction of two shells. In each example considered, explicit formulas are obtained for the pressure transform, and corresponding explicit and relatively simple expressions are given for the various diffraction coefficients associated with the fluid/structure interaction. The general method of solution uses the Wiener-Hopf technique to solve dual integral equations for the acoustic pressure. The plates and shells are modeled by the classical theory of flexure and thin shell theory, respectively. Numerical results of the diffraction coefficients and the redistribution of energy at structural junctions will be presented. [Work supported by ONR.] 
2pSA2. Wiener-Hopf techniques in electromagnetics. Leopold B. Felsen (Dept. of Aerospace \& Mech. Eng., Boston Univ., 110 Cummington St., Boston, MA 02215)

Wiener-Hopf techniques have been applied to a variety of electromagnetic boundary value problems for the past four decades. Examples are presented, together with the techniques used to tackle them. Some questions are then asked concerning the role of Wiener-Hopf model solutions with respect to realistic problem scenarios in electromagnetics or acoustics.

2pSA3. Scattering by ohjects at fluid/solid interfaces. R. V. Craster (Dept. of Theoretical Mechanics, Univ. of Nottingham, Nottingham NG7 2RD, UK)

Canonical diffraction and scattering problems involving fluid/elastic solid interfaces are solved exactly using the Wiener-Hopf technique; the farfield directivities are explicitly identified. When fluid loading is light, there are regions of nonuniform behavior and intense beam formation that occur. In addition, an heuristic method is devised that partly decouples the fluid and solid responses. This leads to accurate results in the light fuid loading limit and allows one to utilize some results directly from elastodynamic problems solved when the fluid is absent.

2pSA4. Calculation of diffraction coefficients for a crack in an anisotropic solid. Gerry R. Wickham (Dept. of Mathematics and Statistics, Brunel Univ., Uxbridge, Middlesex UB8 3PH, UK) and Patricia A. Lewis (Bolton Inst., Bolton BL3, UK)

The diffraction of sound by a semi-infinite planar crack arbitrarily oriented in a homogeneous anisotropic linearly elastic solid is considered. The problem is formulated exactly as an integrodifferential equation with a difference kernel which is the stress tensor corresponding to the fundamental point force solution for the uncracked solid. The solution of this equation is the crack opening displacement (COD) induced by the incident field and this may be expressed in terms of the Wiener-Hopf factors of the Fourier symbol of the kernel. The quantity of physical interest is the diffraction coefficient which is proportional to the Fourier transform of the COD and relates the vector amplitude on an incident ray to the crack edge, to the amplitudes on the diffracted rays. In the general case, the necessary Wiener-Hopf factorization cannot be found explicitly. However, by exploiting the fact that the diffraction coefficient is independent of frequency, it is possible to develop a novel numerical scheme for its evaluation. The exact diffracted field is then analytically expressed in terms of these coefficients. It is shown how the rich qualitative geometrical structure of the diffracted field may be obtained and how the solution reproduces known results for special material symmetries.

\section{3:10-3:25 Break}

2pSA5. On the approximate factorization of scalar and matrix Wiener-Hopf kernels with applications in structural acoustics. 1. David Abrahams (Dept. of Mathematics, Keele Univ., Keele ST5 5BG, UK)

The Wiener-Hopf technique has proved to be an extremely powerful aid to solving problems in diffraction theory, and in particular for acoustic wave scattering. The key step in the procedure is the factorization of the Wiener-Hopf kemel into a product of two functions with (overlapping) semi-infinite regions of analyticity. However, for complex problems, such as those concerned with the interaction between fluids and structures, the representation of the scalar factors can have technical difficulties which make their computation both slow and delicate. Further, many important models of this type give rise to matrix kernels, for which no exact factorization technique has yet been devised. In this paper, a new procedure is presented to obtain approximate but explicit factorizations of both scalar and matrix kernels. As well as being simple to employ both analytically and numerically, the accuracy of the component factors can be increased almost indefinitely with little increase in numerical effort. Further, rigorous bounds on the error of these approximations are easy to find. The method is demonstrated by way of example, and the particular relevance of the new scheme to fluid/structure interaction problems is discussed.

3:50

2pSA6. Acoustic interaction with wedge-shaped structures. Andrew N. Norris (Dept. of Mech. and Aerospace Eng., Rutgers Univ., Piscataway, NJ 08855-0909)

This paper will review recent developments in solving canonical structural acoustics problems involving wedgelike structures composed of plates and membranes. The two cases considered are (i) a membrane held taut over a line constraint, and (ii) two semi-infinite thin plates welded together to form a wedge. The wedge-shaped structures are subject to unilateral fluid loading from an acoustic fluid. The method of Malyuzhinets, which was used in the 1950s for the purpose of dealing with simple impedance-type boundary conditions on the wedge faces, has recently been significantly developed by A.V. Osipov, who has shown how it can be successfully applied to these structural acoustics problems. The central idea of the Malyuzhinets/Osipov method is to express the acoustic pressure using a generalized Sommerfeld integral. similar to a Laplace transform. The main analytical steps in the solution will be discussed, and some numerical results will be presented. The plate structure is particularly interesting because both subsonic flexural and nonleaky supersonic structural waves are excited at the vertex by an incident acoustic wave. Interesting asymptotics emerge when the wedge angle described by the fluid is almost $180^{\circ}$ or $360^{\circ}$. [Work supported by ONR.] 


\section{Contributed Papers}

4:15

2pSA7. Reflection and transmission of structural waves at a corner of an arbitrary angle. Jane B. Lawrie (Dept. of Mathematics, Brunel Univ., Uxbridge UB8 3PH, UK) and I. David Abrahams (Keele Univ., Keele STS SBG, UK)

This talk is concerned with an analytic investigation into the reflection, transmission, and scattering of fluid-coupled structural waves by a corner of an arbitrary angle. The fluid domain is an open wedge, the surfaces of which are described by high-order boundary conditions (that is, containing derivatives with respect to variables both normal and tangential to the boundary). Malyuzhinets (1958) obtained an exact solution for a wedge with impedance faces. However, until the works of Osipov (1994) and Abrahams and Lawrie (1995), little progress was made on adapting his method to problems with more realistic wave-bearing boundaries. The model comprises a compressible fluid wedge bounded by two plane elastic surfaces. An unattenuated surface wave, incident from infinity along one wedge face, is scattered at the apex. Several different edge conditions are discussed, including configurations which excite in-plane plate motions. Explicit application of these constraints allows the boundary value problem to be formulated as two inhomogeneous coupled difference equations which are solved using Malyuzhinets' special functions. An analytical solution is obtained for an arbitrary wedge angle.

\section{4:30}

2pSA8. Visualization of scattered waves resulting from an axial surface wave incident on a discontinuity of a fluid-loaded cylindrical shell. Steven L. Means (Appl. Res. Lab., P.O. Box 30, Penn State Unjv., State College, PA 16804)

On fluid-loaded cylindrical shells there is a wave type that propagates in the axial direction at low frequencies and is characterized by a phase velocity slightly lower than the sound speed of the surrounding fluid. When such a wave is incident on a shell discontinuity, energy is scattered back along the cylinder's axis and into the surrounding fluid. This phenomenon may be modeled by considering a wave propagating along a semi-infinite cylindrical boundary having a given surface impedance attached to a semi-infinite rigid cylinder. This model allows one to use the Wiener-Hopf technique to obtain analytic expressions, in integral form, for the scattered pressure in the surrounding fluid. These expressions are then used to obtain visualizations of the scattered field. Comparisons are made with analogous surface waves scattered by a discontinuity of an elastic plate on an elastic foundation.

\section{4:45}

2pSA9. Hybrid asymptotic-numerical method for evaluating diffraction coefficients. Paul E. Barbone and Isaac Harari (Dept. of Aerospace \& Mech. Eng., Boston Univ., Boston, MA 02215, and Dept. of Solid Mechanics, Mater. and Structures, Tel-Aviv Univ., 69978 Ramat Aviv, Israel)

In complicated structural acoustics problems, the lack of relevant diffraction coefficients often limits the applicability of the geometrical theory of diffraction. A hybrid asymptotic/finite-element method is described that lends itself to the numerical evaluation of diffraction coefficients. It is based on the method of asymptotic patching. The farfield asymptotic expansion of the scattered field is patched to a finite-element interpolation of the field near the point of diffraction. This idea leads to the definition of an asymptotically equivalent boundary-value problem, which is defined on a small domain and therefore is efficiently discretized. The method is demonstrated with an application to diffraction by an imperfect wedge. [Work supported by ONR.]

\section{Session 2pUW}

\section{Underwater Acoustics: Spatial, Temporal and Frequency Dispersion Due to Boundary Scattering in Shallow Water Propagation II}

Peter H. Dahl, Cochair

Applied Physics Laboratory, College of Ocean and Fisheries Sciences, University of Washington, 1013 N.E. 40th Street, Seattle, Washington 98105

Paul C. Hines, Cochair

Defence Research Establishment Atlantic, P.O. Box 1012, Dartmouth, Nova Scotia B2Y 3Z7, Canada

Chair's Introduction-1:20

Invited Papers

1:25

2pUW1. Time and angle spreads due to scattering by bottom roughness and sediment volume inhomogeneity. Darrell $R$. Jackson and Kevin L. Williams (Appl. Phys. Lab., College of Ocean and Fishery Sciences, Univ. of Washington, Seattle, WA 98105)

A bistatic scattering model that treats both roughness and sediment volume scattering is used to infer time and angle spreading for sedimentary seabeds. This model is embedded in a simulation code that predicts the received intensity time series taking account of single-bounce bistatic geometry as well as transmitter and receiver directivity. Roughness scattering is treated in the Kirchhoff approximation without invoking the stationary-phase approximation. Sediment volume scattering is treated in the perturbation ap- 
proximation. Both roughness and volume inhomogeneity are assumed to be random processes with power-law spectra. The relative importance of roughness and sediment volume scattering as spreading mechanisms and the energy loss due to forward scattering are examined for a range of sediment types.

$1: 50$

2pUW2. A measurement and interpretation of the temporal coherence of acoustic continuous wave propagation in the Barents Sea. James H. Miller (Dept. of Ocean Eng., Univ. of Rhode Island, Narragansett, RI 02882), James F. Lynch (Woods Hole Oceanogr. Inst., Woods Hole, MA 02543), David D. Pierce, and Christopher W. Miller (Naval Postgrad. School, Monterey, CA 93943)

As part of the Barents Sea Polar Experiment in the summer of 1992, a continuous wave transmission at $224 \mathrm{~Hz}$ was made for $1 \mathrm{~h}$ from a near-bottom-moored source to a navigated, vertical array separated by approximately $35 \mathrm{~km}$. The waveguide was approximately $200 \mathrm{~m}$ in depth, and its oceanography was dominated by the Barents Sea Polar Front separating the cold, fresh polar water from the warm, saline Atlantic water and a strong thermocline due to surface insolation. Following the methods described by Dahl et al. [J. Acoust. Soc. Am. 83, 2175-2179 (1988)], the variance of the phase rate $\left(\nu^{2}\right)$ is found for single hydrophones, for beam-formed (plane wave) data, and for mode-formed data. The parameter $\nu$ governs the frequency spread and is a measurement of the dynamic effects of the ocean on acoustic propagation. The estimates of $\nu$ are related to measured ocean temperature fluctuations and sea surface conditions from the experiment and compared with estimates from the MIZEX data of Dahl et al. The implications of the measurements for sonar system performance including matched field and matched mode techniques are presented. [Research supported by ONR Code 322, High Latitude Dynamics.]

\section{Contributed Papers}

\section{$2: 15$}

2pUW3. On spatial coherence and angular spread of sound forward scattered from the sea surface: Measurements and interpretive model. Peter H. Dahl (Appl. Phys. Lab., Univ. of Washington, Seattle, WA 98195)

Measurements of the spatial coherence of high-frequency sound forward scattered from the sea surface are discussed, along with a simple interpretive model. The data originate from an experiment off the southern California coastline using the research platform FLIP. Measurements were made using omnidirectional sources suspended from a spar buoy (tethered to FLIP) and a line array mounted on FLIP's hull, with range varying between 500 and $1000 \mathrm{~m}$. The frequency was $20-40 \mathrm{kHz}$ and the roughness parameter $\chi=2 k h \sin \theta$ was $\gg 1$ (where $k$ is the acoustic wave number, $h$ is the mns waveheight, and $\theta$ is the grazing angle); thus the measurements represent high-frequency, incoherent scattering in a single surface bounce channel. The coherence was measured across a line array oriented transverse to the direction of propagation, thereby giving an estimate of the horizontal coherence. Horizontal coherence to the degree of horizontal angular spreading and to the performance of beam forming arrays can be related. The simplest model with which to interpret these results is derived from the high-frequency, large-roughness limit of the Kirchhoff approximation. This model satisfactorily explains the data, and provides basis for a predictive model for horizontal coherence and angular spreading in surface forward scattering. [Work supported by ONR.]

\section{$2: 30$}

2pUW4. High-resolution cross-well tomography measurements. Brian Rapids, Tokuo Yamamoto, Andrew Rogers, and Murat Kuru (Geoacoustics Lab., Univ. of Miami, 4600 Rickenbacker Cswy., Miami, FL 33149)

High-frequency/high-resolution marine cross-well tomography experiments have been conducted at several sites in the waters adjacent to South Florida. Frequencies ranged up to $15 \mathrm{kHz}$ yielding nominal resolution of $10 \mathrm{~cm}$ for the tomographed cross sections. Experiments with several crosssectional geometries determined by depth, range, source location, and receiver location were conducted, including orthogonal sections. These geometries allow determination of both two- and three-dimensional sediment compressional wave velocity structure. This structure may be evaluated for spectral content in both the two- and three-dimensional cases, a key indicator of sediment scattering strength. These spectra are computed for the experimental geometries and presented along side the calculated hydraulic structure. A companion paper being presented at this conference discusses the use of these wave-number spectra as input for modeling acoustic backscatter that was also measured at the cross-well tomography sites discussed here [Rogers et al., J. Acoust. Soc. Am.]. It is shown that cross-well to- mography techniques employed on the ocean floor can provide excellent information for prediction of the acoustic backscattering strength. [Work supported by ONR.]

\section{$2: 45$}

2pUW5. Evaluation of seafloor backscattering strength measured on the Florida continental shelf. Andrew K. Rogers, Tokuo Yamamoto, Murat Kuru, and Brian Rapids (Geoacoustic Lab., Univ. of Miami, 4600 Rickenbacker Cswy., Miami, FL 33149)

Recent experiments off the east coast of Florida have measured bottom backscattering strengths for several bottom types at frequencies of 7.5 and $15 \mathrm{kHz}$. Backscattering strengths are presented as a function of frequency, grazing angle, and azimuthal angle. Coincident with the backscatter measurements, forward scattering measurements and three-dimensional crosswell tomography experiments were conducted to quantify the sediment field. The data and analysis of these are presented in a companion paper given at this conference [Rapids et al., J. Acoust. Soc. Am.]. A newly developed scattering model [Yamamoto, J. Acoust. Soc. Am. (to be published)] has been employed to model the backscattering data. Using the results from the three-dimensional cross-well tomography as input, this model is found to be in good agreement with the data taken. Inversion of the data for sediment descriptors such as aspect ratio, compressional wave velocity fluctuation spectral intensity and spectral exponent, and dip structure has been made, yielding values similar to those obtained wih the in situ measurement by cross-well tomography techniques. These modeling results are detailed in this paper and their significance is discussed. [Work supported by ONR.]

\section{3:00-3:15 Break}

2pUW6. Mode filtering of broadband signals for range-dependent shallow-water environments. Indra Jaya and Mohsen Badiey (Graduate College of Marine Studies, Univ. of Delaware, Newark, DE 19716)

A mode filtering technique is described and applied to broadband signatures obtained from recent shallow-water acoustic experiments [Badiey et al., J. Acoust. Soc. Am. 96, 3593-3604 (1994)]. The technique uses a combination of a wavelet transform and singular value decomposition. The order of the modes is arranged by means of their respective energy levels. Each energy level is directly associated with the eigenvalues of the decomposed signal. The progressions of modal structures in time and frequency are given for various azimuthal propagation paths. Evolution of modal structure with frequency is examined by measuring the extent of the frequency band for each propagation path. It is shown that the pattern of 
the modal structure becomes more complicated for higher modes. Furthermore, from the time-frequency analysis, it can be seen that the existence of a given mode can vary with time and that the medium filters the broadband signal. The mode shape is shown as a function of time, frequency, azimuth, sound speed in the sediment, and other shallow-water waveguide parameters.

\section{3:30}

2pUW7. Arctic ice roughness measurements and implied sound propagation losses. John M. Ozard (Esquimalt Defence Res. Detachment, FMO Victoria, BC VoS 1BO, Canada) and John P. Todoeschuck (407-6 Argyle Ave., St. Lambert, PQ J4P 2H5, Canada)

The prediction of transmission loss is a long-standing problem in Arctic sound propagation. Some earlier estimates failed to predict the observed losses by a factor of 2 . These estimates used levels of ice roughness that were believed to be characteristic of the Arctic. A 1400-km long profile of upward looking sonar from near northem Greenland to the vicinity of the Pole was examined. The statistics of the ice roughness are not stationary over this profile. The ice is rougher near the shore. Thus the transmission loss would vary over the profile depending on the roughness. A range of transmission losses that bracket observed values was found.

\section{3:45}

2pUW8. Scattering of acoustic waves from rigid targets in stratified waveguides. T. Udagawa (Dept. of Phys., Univ. of Texas, Austin, TX 78712) and D. P. Knobles (Univ. of Texas, Austin, TX 78713-8029)

Two complementary methods are proposed for exact numerical calculations of the acoustic field scattered by a rigid sphere in an isovelocity fluid layer overlying a horizontally stratified medium. These two methods are based on the partial wave and Fourier expansions of the medium Green's function. It is shown that the method based on the partial wave expansion is useful for clarifying the physical nature of the approximation involved in the method proposed by Ingenito some time ago [F. Ingenito, J. Acoust. Soc. Am. 82, 2051 (1987)]. 1t is in fact shown that the Ingenito approximation is equivalent to replace the exact medium Green's function by that of the free-field Green's function in obtaining boundary values of the field on the surface of the target. Numerical studies are performed and it is demonstrated that the exact results obtained by using the presently proposed methods are largely different from those obtained by using Ingenito's approximation. In addition more complex cases are examined where the target is nonspherical and the waveguide is bottom limited as is commonly found in shallow water ocean waveguides. The sensitivity of the scattered field to various types of geoacoustic profiles when the target is near the sea floor is also considered.

\section{4:00}

2pUw9. Horizontal refraction of 3-D curvilinear wedge modes. Peter C. Mignerey (Acoust. Div. 7120, Naval Res. Lab., Washington, DC 20375)

For shallow-water acoustic propagation, the wavelength is commensurate with the water depth but short compared to the horizontal extent of the problem. Under these conditions a sloping bottom causes the development of normal modes having wavefronts that are curved in the vertical direction. For simple slopes, such wedge modes have been shown to propagate with cylindrical wavefronts along characteristics in the horizontal plane. This work extends adiabatic wedge mode theory to regions of arbitrary bathymetry by constructing a three-dimensional curvilinear coordinate system that follows the contours of the ocean bottom. The requirement for separation of the depth coordinate from the coupled horizontal coordinates produces a nonlinear differential equation for a potential field. The gradient of this field then gives the depth scale factors and curved shape of the wedge modes. A standard normal mode problem is then solved to obtain the curvilinear wedge modes and a ray-trace method is used to study the horizontal motion of those modes. An overview of the model formulation and some examples will be presented.
2pUW10. Intensity moments of underwater sound reflected by a Gaussian spectrum corrugated surface: Measurements and comparison with a catastrophe thenry approximation. John S. Stroud (Univ. of Cincinnati, Dept. of Radiology, Cincinnati, OH 45267-0579), Philip L. Marston (Washington State Univ., Pullman, WA 99164-2814), and Kevin L. Williams (Univ. of Washington, Seattle, WA 98105)

A surface, manufactured out of Styrofoam, provided a pressure release surface for an underwater, forward-scattering investigation. This surface was a single realization from a population of Gaussian spectrum random rough corrugated surfaces (ms roughness $1.5 \mathrm{~cm}$, correlation length 10 $\mathrm{cm}$ ). A broadband omnidirectional source was operated in the frequency range of $95-400 \mathrm{kHz}$ and a broadband receiver was used to measure sound scattered from the surface. The frequency dependence of the $m$ th higherorder intensity moments $I_{m}$ was measured and compared to theoretical predictions [M. V. Berry, J. Phys. A 10, 2061-2081 (1977)] that $I_{m}$ is proportional to $k^{\nu_{m}}$ (for $m>2$ ), where $k$ is the wave number and $\nu_{m}$ is a twinkling exponent. and that $I_{2} \propto \ln k$. Also, the dependence of $I_{2}$ on distance from the surface was examined at a single frequency utilizing various pulse durations. It is known that far from a surface the wave field will obey Gaussian statistics $\left(I_{2}=2\right)$. For short pulses, however, the statistics of the wave field are strongly dependent upon individual reflections. For longer pulses this is the case as well near the surface, but as one moves away it is shown that the Gaussian limit is approached. [Work supported by ONR.]

\section{4:30}

2pUW11. Effects of scattering-induced frequency spread on Doppler performance. Anthony I. Eller and Ellen M. Daley (Appl. Ocean Sciences Operations, SAIC, 1710 Goodridge Dr., McLean, VA 22102)

Frequency spreading caused by forward scatter from a dynamic rough surface will degrade the performance of active acoustic systems that are attempting to identify slowly moving targets against a reverberation background. This paper addresses the sequence of calculations and the approximations made to the governing physics in a system performance model whose primary output is a measure of target detectability at all locations in a two dimensional grid. The relative frequency spreading resulting from system geometries and motion, active waveform shaping, and boundary interactions are compared and are related to Doppler detection performance as a function of environmental conditions. The received reverberation is characterized by the $Q$ function, which is then convolved with an environmental spreading kernel based on the surface wave power spectral density. The scattering angles and frequency of boundary interaction are determined by shallow water propagation codes.

\section{4:45}

2pUW12. Total seattering cross section of two acoustically coupling balls. Andrey V. Lebedev and Alexander I. Khilko (Hydroacoustic Div., Inst. Appl. Phys., Russian Acad. Sci., 46 Ulyanov str., Nizhny Novgorod 603600, Russia)

The problem of a plane acoustic wave scattering at two acoustically coupled balls is considered. The solution is oblained via the theorem of addition for spherical functions. Asymptotic cases of extremely low- and high frequency are analyzed. It is shown that in a general case in a lowfrequency region total scattering cross section is two times more than for uncoupled balls. In the case of acoustically soft balls the coupling leads to decrease this value due to mutual damping of vibrations via near fields. It was found that in this case there is the optimal ratio of balls radii when the total scattering cross section reaches maximum. In the high-frequency region one can see clearly manifested spatial scales that are conditioned by geometrical sizes of the coupling system. It leads to character oscillations in the frequency dependence of scattering cross section. Maximal influence of the coupling effects in the high-frequency region is manifested when one of the balls is in the shadow of the other. In this case, dominant oscillations are defined by condition $k d=\pi n$ ( " $d$ " is the distance between the balls' centers) and caused by perturbations of the scattering pattern by the shading ball. [Work sponsored by ISF.] 


\title{
Session 3aAO
}

\section{Acoustical Oceanography: Tomography}

\author{
Robert C. Spindel, Chair \\ Applied Physics Laboratory, University of Washington, 1013 N.E. 40th Street, Seattle, Washington 98105
}

Chair's Introduction-8:10

\section{Contributed Papers}

8:15

32AO1. Horizontal refraction tomography for nonadlabatic propagation. A. G. Voronovich and E. C. Shang (CIRES, Univ. of Colorado/NOAA/ETL, 325 Broadway, Boulder, CO 80303)

A scheme of acoustical tomography of the ocean based on measurements of horizontal refraction angles associated with different acoustic modes has been developed recently [A. G. Voronovich and E. C. Shang, J. Acoust. Soc. Am. (to be published)]. Retrieving the 3-D structure of mesoscale inhomogeneities of sound speed proceeds in this case in two steps: (1) determining propagation constants of different modes in the horizontal plane (2-D linear tomography), and (2) retrieving sound-speed profiles basing on a known set of propagation constants at each horizontal point (1-D nonlinear tomography). This tomography scheme was successfully tested in a numerical simulation based on an assumption of adiabaticity of acoustic propagation. "Splitting" of the inverse problem into two stages appeared to be very effective computationally. The present investigation extends this tomography scheme for the situations when the mode's interaction should be taken into account. Now, retrieving the set of propagation constants proceeds iteratively and includes data on sound-speed profiles found at the previous step. For a relatively weak mode interaction which can be considered in a perturbative manner iterations converge after a few steps and the inversion scheme remains very effective. Typical parameters of mesoscale inhomogeneities which can be handled by this iterative approach are estimated. [Work supported by ONR.]

\section{8:30}

3aAO2. Nonlinear tomographic inversion by using the WKB modal condition. E. C. Shang, Y. Y. Wang, and A. G. Voronovich (CIRES, Univ. of Colorado/NOAA/ETL, Boulder, CO 80303)

In the HRT (horizontal refraction tomography) scheme [A. G. Voronovich and E. C. Shang, J. Acoust. Soc. Am. 95, 2851(A) (1994)], the 3-D tomographic inversion is performed in two stages: (1) retrieving the modal wave numbers $k_{m}(x, y)$ in the horizontal plane and (2) reconstructing the sound-speed profile SSP in the vertical at each node of the horizontal plane basing on a set of retrieved modal wave numbers $k_{m}(x, y)$. Usually, a nonlinear approach has to be considered for the 1-D vertical inversion of the second stage. In this paper, the nonlinear inversion by using the WKB modal condition is proposed. The advantages of using WKB modal condition are: (a) it is efficient, the data $k_{m}(x, y)$ can be matched directly to SSP without a model-based calculation (replica); (b) it works even if just a few modes are available; and (c) the error can be analyzed. Some results of numerical simulation are discussed. [Work supported by ONR.]

\section{$8: 45$}

3aAO3. Calculating received time-domain amplitude and phase from 3-D ray tracing results. Mark Grabb and Ted Birdsall (Dept. of Elec. Eng. and Comput. Sci., Univ. of Michigan, 1301 Beal, Ann Arbor, MI 48109)

For long-range sound propagation simulations, ray tracing has many advantages as well as drawbacks. Some of the advantages ray tracing offers are 3-D implementation with present-day computers and the capability of propagating only the acoustic field that will contribute to a single early arrival. Well-known drawbacks to ray tracing computations are the neglect of diffraction effects, infinite-amplitude results at caustics, and the often quoted "high-frequency approximation." However, other simulation techniques have advantages and drawbacks as well, suggesting the community should use many of the available propagation techniques and build strong conclusions through assimilation of results. Here, a ray-endpoint density method is investigated for calculating the time-domain amplitude of a received early arrival. Also, computation of the received time-domain phase is studied. The methods are investigated using 2-D range-invariant, 2-D range variant, and 3-D time and space variant computational ocean sound-speed models. The ray-endpoint density method is compared against normal mode, PE, and dynamic ray tracing results. [Work supported by ONR-ASSERT.]

\section{9:00}

3aA04. Nonlinear FM short-duration waveform design procedure for travel-time measuring systems. Mark Grabb and Ted Birdsall (Dept. of Elec. Eng. and Comput. Sci., Univ. of Michigan, 1301 Beal, Ann Arbor, MI 48109)

Many oceanographic experiments and sonar systems require a shortduration, low-frequency, large bandwidth interrogation waveform for the purpose of obtaining precise travel-time measurements. The waveforms employed can typically be classified into three categories: short pulse, digital burst, or FM sweep (chirp). The FM sweep is the most flexible waveform class in that it can have arbitrary duration and amplitude while supplying desirable matched filter output properties (high resolution and low time-domain sidelobes). A design procedure has been developed to produce nonlinear FM burst waveforms that closely obtain a "desired" energy spectral density and hence matched filter output. A transminter-toreceiver system analysis of the waveform has been conducted that includes a linear model of the transducer. The system analysis unveils the significant benefits of knowing the transducer characteristics precisely as well as uncovers the need for a transmitter clock rate that is significantly higher than the waveform's Nyquist rate. [Work supported by ONR-ASSERT.] 


\section{9:15}

3aAO5. Inversion of broadband multitone data from the Yellow Shark experiments. Peter Gerstoft, Jean-Pierre Hermand (SACLANT Undersea Res. Ctr., 19138 La Spezia, Italy), and Taco van der Leij (Ctr. for Technical Geoscience, Delft Univ. of Technol., Delft, The Netherlands)

The estimation of forward model parameters-geometric, geoacoustic, and ocean sound-speed - by the inversion of acoustic field observations is considered. During autumn '94 and spring '95 an exhaustive set of broadband acoustic data and environmental data was obtained over a shallowwater area in the Mediterranean Sea [Hermand et al., "The Yellow Shark Broadband Inversion Experiments" (to be published)]. In this paper, inversion results of a mildly range-dependent transect southeast of the Island of Elba are presented. The inversion is carried out from multitone data (in the frequency band $200-1600 \mathrm{~Hz}$ ) received on a $62-\mathrm{m}$ vertical array from a fixed acoustic projector deployed at different ranges $(4.5-15 \mathrm{~km})$. Hydrographic data measured during the acoustic transmissions and coring and seismic profiling of the sediments along the acoustic track are utilized to control and verify the inversion process. Global optimization through a directed Monte Carlo search based on genetic algorithms and the Bartlett objective function is applied. All geometric parameters are well determined and also a range-dependent geoacoustic model and the ocean soundspeed profile are estimated. The use of observarions at multiple frequencies provides considerable stability for the estimated parameters.

\section{$9: 30$}

3aA06. Data analysis and inversion of basin scale sound propagation in the Western Mediterranean Sea. Hidetoshi Fujimori, Iwao Nakano, Satoshi Tsukioka (Japan Marine Sci. and Technol. Ctr., 2-15 Natsushima-cho, Yokosuka 237, Japan), James F. Lynch, and Arthur E. Newhall (Woods Hole Oceanogr. Inst., Woods Hole, MA 02543)

In 1994, an ocean acoustic tomography experiment was performed in the Western Mediterranean Basin. It is a pilot tomography project for monitoring in this area. This project is called THETIS and constructed by European tomography groups. The S-Tether system was deployed in this experiment. This system consists of a $400-\mathrm{Hz}$ tomography transceiver made by Webb Res. Co. and a surface buoy developed by WHOI and JAMSTEC. The received data from four other transceivers to the S-Tether were analyzed. The distances from S-Tether to four communicated transceivers were about $250-450 \mathrm{~km}$. Sound was propagated four times a day from late January to late October in 1994. In the Mediterranean Sea, the sound velocity increases with depth from the surface down to the bottom and sound propagates in the surface sound channel. A simple inversion was used for this analysis. This method is effective for this surface sound channel. The time series of the temperature distribution was obtained. These are the vertical profiles averaged along the horizontal ranges. Seasonal changes are observed from the temperature time series. In the winter, a flat temperature distribution was covered from the surface to the bottom. It is considered to be sinking, which is a typical phenomena in the Mediterranean Sea.

\section{9:45}

3aA07. Acoustic tomography in the Western Mediterranean from a moving ship. Dmitry Yu. Mikhin, Sergey V. Burenkov, Yury A. Chepurin, Valerii V. Goncharov, Vladimir M. Kurtepov, Viktor G. Selivanov (P. P. Shirshov Oceanogr. Inst of the Russian Acad. Sci., Moscow 117851, Russia), and Oleg A. Godin ${ }^{a)}$ (NOAA/Atlantic Oceanographic and Meteorological Lab., Miami, FL 33149)

A moving ship tomography experiment was carried out in the Western Mediterranean in 1994. Broadband sound signals were emitted by six moored transceivers deployed by IfM (Kiel, Germany), IFREMER (Brest, France), and WHOI (Woods Hole, USA), in the framework of the THETIS-2 project and recorded at a hydrophone deployed from a drifting research vessel. The acoustic measurements were supplemented with a detailed CTD survey. The data processing technique used made it possible to compensate for the Doppler shift due to vessel drift, measure the channel pulse response and estimate the arrival angles of different rays. The arrival pattern proved to be consistent with numerical predictions using adiabatic normal modes and ray theory. The first results of tomographic inversion for a single vertical slice are presented. The travel times of early raylike arrivals and final cutoffs constitute the data set for inversion. For distances over $300 \mathrm{~km}$, late modal arrivals were resolved, identified and incorporated into the data set. An alternative inversion approach based on matching the overall arrival pattern is discussed and compared with traditional schemes. [Work supported by ISF, INTAS, and RBRF.] ${ }^{\text {a) }}$ On leave from P. P. Shirshov Oceanography Institute, Moscow, Russia.

\section{0:00-10:15 Break}

10:15

3aAO8. Simulations of acoustic tomography of ocean currents in a coastal region. Oleg A. Godin a) (NOAA/Atlantic Oceanographic and Meteorological Lab., 4301 Rickenbacker Cswy., Miami, FL 33149) and Dmitry Yu. Mikhin (P. P. Shirshov Oceanogr. Inst. of the Russian Acad. Sci., Moscow 117851, Russia)

A new, full field approach to acoustic monitoring of currents in the ocean-matched nonreciprocity tomography (MNT)-was recently put forward [Godin et al., in Full Field Inversion Methods in Ocean and Seismo-Acoustics, edited by O. Diachok et al. (Kluwer, Dordrecht, 1995), pp. 26I-266]. The approach is based on inverting nonreciprocity in phase of a cw signal measured at a set of points in a given vertical plane. MNT was designed to overcome the problems with rays resolution and identification which make the traditional current tomography technique inapplicable in coastal regions. Results of numerical simulations of suggested MNT field experiments in the Strait of Florida will be presented. The possibility to increase resolution and robustness of the inversion with few transceivers by using wideband signals is considered. The amount of $a$ priori environmental information required for an unambiguous currents inversion by MNT is evaluated. The possibility of simultaneous measurements of both horizontal components of current velocity is also addressed. Finally, the effects of surface and internal waves on the acoustic monitoring of ocean currents are analyzed. [Work supported by NRC and RBRF.]

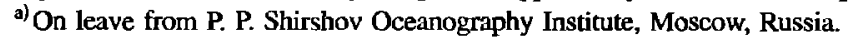

\section{0:30}

3aAO9. Analytical investigation of ray chaos in an underwater acoustic system. Zhong-Yue Jiang, Todd Pitts, and James F. Greenleaf (Biodynamics Res. Unit, Dept. of Physiology and Biophysics, Mayo Clinic and Foundation, Rochester, MN 55905)

It has previously been shown that acoustic ray paths in rangedependent ocean models exhibit chaotic behavior. Most of the investigations into the ray chaos phenomenon have been primarily numencal in nature. The objective of this report is to study theoretically the existence of ray chaos in a parabolic ray system with an analytically prescribed soundspeed model consisting of a double-channel profile perturbed by a typical periodic range-dependent disturbance. The perturbed Hamiltonian ray system is studied analytically via Melnikov's method. It is shown that, under certain conditions, ray trajectories in some regions of the Poincare section are equivalent to trajectories of the horseshoe map no matter how small the corresponding perturbation is. These conditions are sufficient for ray chaos and easily satisfied, thus explaining why double-channel propagation is very likely to exhibit chaotic behavior. [Work supported by CA43920 NIH.] 
10:45

3aAO10. Paleo-ocean acoustics: The historical variation of low-frequency sound absorption in the world's oceans and the implications for sea mammals. David G. Browning (Browning Biotech, 139 Old North Rd., Kingston, RI 0288I) and Robert H. Mellen (Kildare Corp., New London, CT 06320)

Sound is the primary method of communication in the ocean. The absorption of sound in seawater limits the range and fidelity of communication. This absorption is due to three chemical relaxation mechanisms which, of course, would vary with any change in their constituents, such as sulphur, magnesium, or carbon; or any environmental parameters such as temperature. At the low frequencies (below I $\mathrm{kHz}$ ) required for long-range communication, the principal absorption mechanism is also a $p \mathrm{H}$ dependent buffered boric acid reaction. In tum, the $p \mathrm{H}$ in the ocean is controlled by the amount of dissolved carbon dioxide. All these factors could vary during the Earth's evolution. Estimates are made of the possible changes in absorption and, hence, ocen communicalion conditions over the ages. based on the evolution of ocean as put forth by Holland [H. D. Holland. B. Lazar, and M. McCaffrey, Nalure 320, 27-33 (6 March 1986)] and others, with emphasis on the time scale, approximately 50 million years, of ocean mammal development.

\section{1:00}

3aAO11. Investigation of sound absorption in the deep sea using a submergence vehicle. Toshio Tsuchiya, Yasutaka Amitani, Hiroshi Ochi, Takuya Shimura (Japan Marine Sci. and Technol. Ctr., 2-15 Natsushima-cho, Yokosuka 237, Japan). Toshiaki Kikuchi, and Masaaki Umezawa (Natl. Defence Acad. of Japan, Yokosuka 239, Japan)

For the design of underwater acoustic equipment, it is very important to estimate the saund absorption, especially since the longer the propagation range, the greater the effect of sound absorption. Generally, the equations of Thorp, Schlkin, and Marsh and Francois and Garrison are often used for the determination of sound absorption, but these equations yield values that are different from each other. So, the signal level from the data transmitter (center frequency: $20 \mathrm{kHz}$ ) of the deep submergence vehicle "Shinkai 6500" were measured in detail on the mother ship. The dives for this investigation were carried out at a maximum depth of $6500 \mathrm{~m}$ in the northwest Pacific area. It is found that Francois and Garrison's equation was comparatively suitable for the measured data.

\section{1:15}

3aA012. Remote ocean sensing by parametric array. Konstantin $A$ Naugolnykh (Environ. Technol. Lab., Boulder, CO 80303) and Igor B. Esipov (N. Andreyev Acoust. Inst., Moscow 117036, Russia)

Parametric arrays launched by P. J. Westervelt as a result of an investigation of nonscattering of sound by sound [P. J. Westervelt, J. Acoust. Soc. Am. 35, 535-537 (1963)], have become familiar in the practice of marine research. The narrow beam of a wide frequency band parametric signal in the low-frequency range makes an attractive parametric array application for ocean sensing on a long-range acoustic pathway. Results of an experimental application of a high power narrow beam parametric array in a variety of environmental ocean conditions, that is, shallow water, long-range (up to $1000 \mathrm{~km}$ ) signal propagation, and synoptic ocean eddy remote sounding, are considered in this paper. This space pattem of the parametric antenna field is shown to be essentially changed with the direction of the ocean eddy probing. Horizontal refraction occurs when a parametric signal passes through the eddy. This shows the possibility of a long distance tomographic analysis of mesoscale environment patterns of the ocean by a high-directed parametric array. The problem of parametric array application on elongated pathways in the ocean is discussed, the advantage of the parametric array with respect of a conventional one in the ocean waveguide is considered also. [Work supported by ISF grant MH 1300 and RFFI grant 95-02-06353a.]

\title{
Session 3aEA
}

\section{Engineering Acoustics: Measurements and Noise Control}

\author{
Yves H. Berthelot, Cochair \\ Georgia Institute of Technology, School of Mechanical Engineering, Atlanta, Georgia 30332 \\ Robert D. Corsaro, Cochair \\ Naval Research Laboratory, Code 7135, Washington, DC 20375-5350
}

\section{Contributed Papers}

\section{8:00}

3aEA1. A broad frequency anechoic lining system. N. Scott Emery (Vector Res. Co., Inc., Ste. 700, 2101 E. Jefferson St., Rockville, MD 20852). Claude Ledoux (Naval Undersea Warfare Ctr., Newport, RI 02841), and Eric Buchanan (Seaward Intl., Clearbrook, VA 22624)

Testing in indoor underwater acoustic test tanks can be limited by ambient noise and by reflections from the tank's surfaces. The severity of reflection problems is related to a tank's dimensions and the acoustic reflectivity of the tank's sufaces. To reduce reflection problems, a tank may be enlarged, but in most cases, this solution is not acceptable. An altemative solution is to modify the reflectivity of the tank's surfaces by adding an anechoic lining to the tank. A broad frequency ( 1 to $100 \mathrm{kHz}$ ) anechoic lining system was designed and developed for the 700000 gallon Acoustic Test Facility at the Naval Undersea Warfare Center in Newport, RI. To accomplish broad frequency absorption, iwo parallel gradual transition absorbers are used: low-frequency, triangular wedges arranged in rows 6 in. apart and high-frequency molded pyramids spaced approximately 1 in. apart. Both the wedges and pyramids are fabricated from specially formulated, microvoided-polyurethane composites. The system is designed to provide 8 to $33 \mathrm{~dB}$ of echo reduction between 1 and $100 \mathrm{kHz}$. Measured prototype performance exceeded $10 \mathrm{~dB}$ at frequencies above $1.8 \mathrm{kHz}$. System acoustic design and materials development are discussed. The results of acoustic testing are presented and compared to design predictions. 
3aEA2. Evaluation of a computationally assisted low-frequency calibration technique in an acoustic tank. Claude A. LeDoux and Regina M. DuBord (Naval Undersea Warfare Ctr., Div. Newport. Newport, RI 02840)

Acoustic tank calibration of transducers using low-frequency pulsed sinusoidal inputs (below $500 \mathrm{~Hz}$ ) in water has generally been impossible, due to strong, variable signal reflections from the tank walls and air-water interface that corrupt measurements before the transducer response reaches steady state. Since the large tank size needed to overcome this difficulty is impractical, a computational scheme has been investigated for predicting these steady-state responses. Specifically, Prony's method, applied to the echo-free portion of a response signal, has been employed to generate a mathematical signal model which theoretically can be used to extrapolate the response to any later time, as suggested by Beatty et al. [J. Acoust. Soc. Am. 63, 1782-1794 (1978)]. An experimental assessment of this approach, performed in the Naval Undersea Warfare Center acoustic tank, as well as experimental justification of its validity, will be presented.

\section{8:30}

3aEA3. Development of a scanning laser Doppler vibrometer for imaging the surface velocity of a submerged vibrating object of arhitrary geometry. Jayme J. Caspall, Michael D. Gray, Gary W. Caille (Undersea Res. Prog. Office, Georgia Tech Res. Inst., Atlanta, GA 30332-0810), Jacek Jarzynski, Peter H. Rogers, and George S. McCall II (Georgia Inst. of Technol., Atlanta, GA 30332-0405)

An underwater scanning laser Doppler vibrometer (USLDV) was developed as part of a system for imaging the vibrations of submerged surfaces of arbitrary geometry. USLDV allows for measurement of in-plane and out-of-plane velocity components with scan resolution limited typically by the resolution of the positioning system $(<1 \mathrm{~mm})$. The Georgia Tech system features a compact underwater probe mounted on an acoustically transparent mounting truss whose position and orientation is computer controlled. The system was designed with special consideration of the measurement of compliant surfaces underwater. Characteristically low impedance and locally reacting, compliant surfaces are sensitive to field perturbations, such as those which may be generated by the measurement apparatus. Small scattered fields can generate sizable local surface motion, leading to measurement error. The design of the underwater system components was performed in consideration of the perturbation sensitivity of compliant surfaces as well as such issues as sound-induced modulation of the index of refraction, and vibration of the probe head due to incident sound and/or the motion of the positioning truss. [Work supported by ONR.]

\section{8:45}

3aEA4. Investigation of Lamb waves in copy paper using laser ultrasonics. Mont A. Johnson, Yves H. Berthelot (School of Mech. Eng., Georgia Inst. of Technol., Atlanta, GA 30332-0405), Pierre Brodeur, and Joe Gerhardstein (Inst. of Paper Science and Technol., Atlanta, GA 30318-5794)

At present, the paper industry lacks an on-line, noncontact quality control system. Several studies indicate that there are strong correlations between paper strength and elastic stiffness properties. These elastic properties or elastic coefficients can be determined by measuring the velocities of propagation of various ultrasonic waves. Lamb waves, for instance, propagate in paper and appear to be sensitive to paper strength. Experiments have been conducted with a laser-based system to both generate and detect ultrasonic Lamb waves in copy paper. Results obtained regarding the relationship between the generation laser spot size, power density, and ultrasonic signal strength are presented. Paper damage is assessed at the generation spot. Phase velocity dispersion curves are produced for those modes that have sufficient signal-to-noise ratio. These dispersion curves are then companed to those predicted by ortotropic plate theory. [Work supported by OIP-GT.]
3aEA5. Acoustic material characterization by laser interferometry. R. Lance Willis, T. Shane Stone, and Yves H. Berthelot (School of Mech. Eng., Georgia Inst. of Technol., Atlanta, GA 30332-0405)

An interferometric system consisting of five independent probes cabable of measuring in-plane or out-of-plane surface motion of a vibrating sample at five different points simultaneously has been designed, constructed, and tested. A description of the design, measurement capabilities, and performance specifications will be given. The system utilizes optical fibers and compact probe design $(30 \mathrm{~mm} \times 15 \mathrm{~mm} \times 9 \mathrm{~mm})$ to enable close spacing measurement points. Signal demodulation is performed by phaselocked loops allowing real-time measurements. The system is designed to test voided polymer samples excited by a shaker in the $100 \mathrm{~Hz}$ to $5 \mathrm{kHz}$ range. It is calibrated for steady state and transient excitation of the sample. Results include measurements of the damping constant of the sample in the time domain for various frequencies of excitation. [Work supported by the Office of Naval Research, Code 334.]

\section{9:15}

3aEA6. An inverse acoustic problem in the presence of a mean flow. Sheryl M. Patrick (Dept Aero. and Mech. Eng., Boston Univ., 110 Cummington St., Boston, MA 02215) and Hafiz M. Atassi (Univ. of Notre Dame, Notre Dame, IN 46556)

Recent research has shown the feasibility of performing inverse aeroacoustic problems for streamlined bodies. The unsteady pressure on a flatplate airfoil, due to a convected vortical disturbance in the mean flow, can be recovered from the far-field radiated sound. The present paper extends this analysis to oscillating airfoils in a uniform mean flow. In this case, the oscillating airfoil creates an unsteady pressure field on the airfoil surface. The inverse problem, then, is to determine the surface pressure from the radiated sound. For the oscillating airfoil problem, the normal pressure gradient does not vanish along the airfoil surface, rendering the inversion process more complex than for the gust problem, however it is still feasible. This paper also compares the oscillating airfoil application to acoustic holography. While the two problems are similar mathematically, farfield input data are, in general, not sufficient for acoustic holography applications while they are sufficient for the inverse aeroacoustic problem.

\section{9:30}

3aEA7. Multilayered model for near-field acoustical holography of sound sources with hot surfaces. Martín Gutiérrez V. (Inst. of Acoust., Universidad Austral de Chile, Casilla 567, Valdivia, Chile)

Since near-field acoustic holography (NAH) is a powerful tool to investigate the sound radiation of a wide range of sources immersed in a homogeneous medium, a corrective model for the presence of inhomogeneities in the near field, where the hologram is performed, is presented. Strong temperature gradients modify the propagation of the sound wave, because they modify the density of the medium and then the sound speed. Many models were developed to take into account this and other effects; most of them were proposed in the atmospheric sound propagation area. The actual model was developed to minimized the effect of temperature gradients in the near field. An early model, previously presented [M. Gutiérrez and J. Arenas, J. Acoust. Soc. Am. 96, 3263(A) (1994)], based on the spatial transformation of sound fields technique, was modified in order to obtain a new mathematical expression based on a multilayered Green's function. Some theoretical sources were numerically tested with the new model, which supposed a disturbance during heat transmission to the free air by an "omnidirectional" heat source. The comparative results between NAH theory and the proposed model will be shown. 
10:00

3aFA8. High-power ultrasound measurement. G. S. K. Wong and L. Wu (Acousi. Standards, Inst. for Natl. Measurement Standards, Natl. Res. Council Canada, Ottawa, ON KIA 0R6, Canada)

A system for the absolute measurement of high-power ultrasound in the range from 0.5 to $30 \mathrm{~W}$ is described. The theory of the system is based on measuring the ultrasound radiation force exerted on a conical-float suspended in water. The system is fully automated with the aid of the IEEE488 bus under the control of a computer. Measurement procedures and an uncertainty audit representing a confidence level of approximately $95 \%$ are presented.

\section{0:15}

3aEA9. Determination of acouslic center correction for type LS2aP condensor microphones. Randall P. Wagner (Natl. Inst. of Standards and Technol.. Bldg. 233, Rm. A 147, Gaithersburg. MD 20899) and Victor Nedzelnitsky (Natl. Inst. of Standards and Technol., Gaithersburg, MD 20899)

Acoustic center corrections for microphones are necessary for accurate free-field calibration by the reciprocity technique. Such corrections for several pairs of the relatively new type LS2aP microphone [IEC Std. 1094-1: 1992] were obtained by utilizing the theoretical inverse relationship between the sound pressure amplitude at the receiving microphone and the distance between the acoustic centers of the source and receiving microphones. For a nominally $10-\mathrm{V}$ sinusoidal rms excitation of the source microphone, source-to receiver voltage ratios were measured with a dynamic signal analyzer at $500-\mathrm{Hz}$ intervals in the extended frequency range (2 to 50) $\mathrm{kHz}$. This procedure allowed all the data for a microphone pair to be gathered within several hours as a function of microphone diaphragm separation at $10-\mathrm{mm}$ intervals from 101 to $311 \mathrm{~mm}$. At each frequency, these ratios were corrected for atmospheric effects, including attenuation of sound, and then fit to a straight line (ratio versus diaphragm separation. Acoustic center corrections were calculated from the derived values of the fit parameters. These corrections agree with appropriately scaled values [IEC Publicalion 486-1974] for "l-in." microphones with recessed diaphragms. Physical phenomena that cause small deviations from the linear fits will be discussed along with uncertainty estimates, and effects of spatially fruncating data.

\section{0:30}

3aEA 10. Theoretical prediction of the natural frequency of electrostatic ultrasonic transducers. Li-Feng Ge and L. Finkelstein (Dept. of Elec., Electron. and Information Eng., City Univ., London ECI, England)

Electrostatic airborne ultrasonic transducers with an air-gap structure are widely used in automatic ranging, acoustic imaging, NDE, etc., so that there is an increasing need to develop a reliable model for their optimal design. A Helmholtz resonator model and a plate-under-tension model were presented successively in recent years, but both are still not very satisfactory. In this paper, an electrostatic transducer is treated as a thin plate supported by an air spring, in other words, its diaphragm is viewed as a plate situated on an air cushion. An interesting point revealed by this model is that its fundamental frequency stems from the air-gap spring, and the higher resonant frequencies are caused by the combined contributions of the bending stiffness of the diaphragm, in-plane tensile forces applied to the diaphragm, and the air-gap stiffness. The model is applied to predict the nalural frequency of a typical V-grooved transducer with a 8 $\mu$ m-thick Mylar diaphragm and a 0.5-mm pitch air gap. The first resonant frequency predicted by this model is $50.8988 \mathrm{kHz}$, and its nominal measured values are $51 \mathrm{kHz}$ and $52 \mathrm{kHz}$, respectively, as reported by some researchers. [Work supported by the UK Royal Society.]
3aEA11. Micromachined ultrasonic transducers (MUTs): Theory and experiment. Igal Ladabaum, B. T. Khuri-Yakub (E. L. Ginzton Lab., Stanford Univ., Stanford, CA 94305), and Dimitri Spoliansky (Ecole Normale Superieure, 75005 Paris, France)

The successful modeling and fabrication of capacitive ultrasonic air transducers is reported. Emission and reception in air at 11.4, 9.2, and 3.1 $\mathrm{MHz}$ is demonstrated. Furthermore, transmission experiments through airglass-air ( $70 \mathrm{~dB}$ of signal loss) at 3.8 and $5.1 \mathrm{MHz}$ are reported. The transducers are made using surface micromachining techniques, which enable the realization of center frequencies ranging from 1.8 to $11.6 \mathrm{MHz}$. A theory explaining both the static and dynamic operation of the devices is presented. In addition to agreeing well with the experiments, the theory predicts that displacements on the onder of $10^{-3}$ angstroms (with potential for $\mathrm{I}^{-5}$ angstroms) are detectable with a 20-dB signal-to-noise ratio. Such detection sensitivity is shown to yield air transducer systems capable of withstanding over $100 \mathrm{~dB}$ of signal attenuation, a figure of merit that has significant implications for ultrasonic imaging, nondestructive evaluation, gas flow and composition measurements, and range sensing. [This work has been supported by a grant from ONR.]

\section{1:00}

3aEA12. Smart area actuator with integrated accelerometer array. Joseph A. Klunder and Robert D. Corsaro (Naval Res. Lab., Code 7130, Washington, DC 20375-5350)

This paper describes the design and evaluation of a very low cost high-sensitivity accelerometer included in a large area actuator. Recently MSI has begun commercial production of a 1-3 composite transducer material using an injection molding approach. This actuator material consists of a large number of transducer rods, which would typically be combined to form an actuator. NRL recognized that an incidental number of these rods could instead be converted for use as an imbedded accelerometer array, at little additional cost. This paper describes the design and performance of accelerometers formed using this process, and issues related to their proximity to the actuator. Such combination transducers are particularly needed in active control and smart-materials applications, but additionally would find use in advanced underwater, aerospace or robotic sensing applications.

\section{1:15}

3aEA13. Reduction of structure-borne noise using an air-voided elastomer. Sung H. Ko (Naval Undersea Warfare Ctr. Detachment, New London, CT 06320)

A theoretical model was developed to evaluate the reduction of structure-borne noise which is generated by a line force applied on an infinite plate using an elastomeric baffle. The vibrating plate is covered with an elastomeric baffle layer to reduce the noise generated by the structural vibration. The vibrating plate is perfectly bonded to the elastomeric baffle. The outer surfaces of the vibrating plate and the elastomer baffle are in contact with air and water, respectively. The analysis for modeling is based on the theory of elasticity and pertinent boundary conditions. Effects of various parameters such as baffle layer dimensions and material properties on the noise reduction are presented.

\section{1:30}

3aEA14. Helmholtz resonator in the induction system of a spark-ignition engine with production versus prototype manifolds. A. Selamet, S. H. Yonak (Dept. of Mech. Eng. and Appl. Mech., 120 W. E. Lay Automotive Lab., Univ. of Michigan, Ann Arbor, MI 48109-2121), and J. M. Novak (Ford Motor Co., Dearbom, MI 48121)

Following an earlier work [A. Selamet and J. M. Novak, J. Acoust. Soc. Am. 97, 3393 (1995)], the present study investigates the performance of a Helmholtz resonator installed in the induction system of a Ford $3.0 /$ V6 Vulcan engine in a dynamometer test facility. Earlier findings with the production manifold are compared with the current results obtained with a 
modified prototype manifold in an atternpt to determine the extent of multidimensional wave dynamics. The induction system is instrumented with fast-response piezoresistive transducers to capture the temporal and the spatial variation of air pressure. Measured insertion loss characteristics of the silencer are then discussed in comparison with the predictions from a nonlinear computational fuid dynamics approach in the time domain.

\section{1:45}

3aEA15. Reference sensor selection for automotive active noise control applications. Kevin D. LePage, P. J. Remington, Alan R. D. Curtis (Bolt Beranek and Newman Inc., 70 Fawcett St., Cambridge, MA 02138), and Scott Knight (Bolt Beranek and Newman Inc., Union Station, New London, CT 06320)

BBN has obtained a comprehensive set of road noise measurements for the purpose of investigating the potential performance of an automotive active noise control application. Measurements were collected over a total of 20 reference sensors on or near the suspension and four residual sensors in the cabin. Analysis shows that while the reference sensors can account for much of what is received at the microphones, some of the reference sensors provide more residual information than others. For control applications the optimum reference sensors for the available number of input channels are desired. Two reference sensor performance metrics are compared. The first is a suboptimal greedy algorithm where reference channels are iteratively selected via an orthogonalization procedure. The second technique is an exhaustive search over candidate reference sensors sets where the coherent power is maximized. Results show that the greedy procedure generally identifies the majority of the sensors shown to be optimum through the exhaustive search. [Work supported by ARPA.]

\section{2:00}

3aEA16. Identification of a space noise control model and active noise control. Zhong Yan Jiong, Yue Kai, and Zhang Zhen Shan (Dept. of Automatic Control, Univ. of Sci. and Technol., Beijing 100083. People's Republic of China)

In order to get the mathematic model of a noise control system in space, which is the impulse response of a speaker-microphone system, a square impulse modulated by a single frequency signal has been used. In the paper, a pseudorandom correlation identification method is used to get the impulse response of a MIMO speaker microphone system in the spatial field. The dynamic process and the time delay of sound waves in the spatial field and speaker coils are presented exactly. The model is used for digital simulation and a space sound experiment using a developed adaptive filter algorithm presented by the authors which is the recursive algorithm with interlacing cycle and increasing phase. The sampled noise signal from the motor driver cabin is used as the input of the system. The simulation and the experiment have proved the correctness of the model and the effectiveness of the developed adaptive fillter algorithm.

\title{
Session 3aNSa
}

\section{Noise: Acoustical Instrument Calibration-Fact and Fallacies}

\author{
John P. Seiler, Chair \\ U.S. Department of Labor, Mine Safety and Health Administration, P.O. Box 18233, Cochran Mill Road, \#147, \\ Pittsburgh, Pennsylvania 15236
}

Chair's Introduction-8:30

\begin{abstract}
It is a well-known axiom that it is necessary for accurate calibrated acoustical instrumentation to be used in order to achieve accurate and repeatable measurements. The term "calibration" has different meanings to different people. The purpose of this session is to examine the topic of acoustical instrument calibration from a number of viewpoints. These viewpoints come from scientists, engineers, lawyers, and industrial hygienists who are acoustical instrument evaluators, manufacturers, and users. Perhaps attendees will come away from the session with a better understanding of the term "calibration," or at least will give it some thought the next time they have an instrument calibrated.
\end{abstract}

\section{Invited Papers}

\section{8:40}

3aNSa1. Primary standards and instrument calibration: A national laboratory perspective. Victor Nedzelnitsky (Natl. Inst. of Standards and Technol., Sound Bldg. 233, Rm. A147. Gaithersburg, MD 20899-0001)

In every advanced industrial nation, urgent needs for accurate, impartial measurement capabilities without unnecessary duplication of laboratories have led to the establishment of a national primary metrology and standards laboratory. These laboratories conduct international comparisons of calibrations and measurements intended to demonstrate sufficiently close agreement, or to reveal and to resolve unexpected discrepancies, among results from different nations. Such comparisons must precede, accompany, and continually sustain agreements reganding legal metrology and product conformity assessment, which are essential to the fair and successful conduct of intemational trade. These laboratories also provide scientific and technical support to the development of international standards, as well as the domestic standards of their respective individual nations. A national laboratory typically meets the critical needs of that nation's private and public calibration laboratories for state-of-the-art measurement services through an instrument calibration hierarchy comprising direct or implied chains of "traceability" to the national laboratory. Selected, consequential international comparisons of electroacoustical calibrations from national laboratories are discussed. Examples of research in acoustical metrology at NIST and the measurement services provided to its customers are described. 
3aNSa2. Calibration: The legal perspective. Robert W. Aldrich (U.S. Dept. of Labor, Office of the Solicitor, Mine Safety and Health Div., 4015 Wilson Blvd., Arlington, VA 22203)

Several federal agencies have developed regulations to protect employees from health hazards in the workplace by establishing time-weighted average exposure limits. In order to ensure that exposures remain within acceptable limits, regulations have also included criteria for conducting accurate sampling. Accurate measurements of exposure can be obtained only when the instruments used are functioning properly and have been calibrated to the manufacturer's specifications. Inaccurate measurements can lead to not only incorrect or unrepresentative worker sampling, but also can result in ineffective action by the employer if it becomes necessary to take corrective actions to reduce exposures to allowable levels. As a result, from a legal point of view, measuring instruments must be properly calibrated and maintained before they are used to measure worker exposures. In addition, the issue of instrument accuracy is important because courts have determined that in order for measurements to be considered "legally certain," they must be accurate to within a $95 \%$ confidence.

3aNSa3. Acoustic instrument performance verification-The manufacturer's perspective. Jonathan M. Blick (Quest Technologies, 510 S. Worthington St., Oconomowoc, WI 53066)

The calibration and testing of acoustical instruments by a manufacturer is primarily a quality control function. Design parameters are differentiated from production test parameters during the design phase. Design parameters are inherent in the final product and may or may not be tested during production. Production testing is performed to ensure product uniformity within tolerances set by the manufacturer, many of which are dictated by national and international standards. Type testing by a third party is often used as a means of verifying compliance to standards, often at the request of customers. Independent testing is also beneficial as a means of verifying in-house test procedures.

3aNSa4. Acoustical instrument calibration: A regulatory agency viewpoint. Michael A. Crivaro (U. S. Dept. of Labor, Mine Safety and Health Administration, P. O. Box 18233, Cochrans Mill Rd., Pittsburgh, PA 15236)

The Mine Safety and Health Administration (MSHA) has the responsibility of protecting the hearing of the nation's miners. Within MSHA, the Office of Technical Support has determined a method of assuring that the noise dosimeters and acoustical calibrators used to conduct noise surveys provide an accurate representation of the miner's noise exposure. The method is comprised of three components. First, the dosimeters and calibrators are evaluated based on recognized standards. Second, each calibrator and dosimeter is calibrated annually in a laboratory using instrumentation with accuracies traceable to the National Institute of Science and Technology. Finally, mine inspectors who conduct the noise surveys are trained on using their instruments correctly including performing a field calibration both before and after the noise exposure measurement.

\section{0:00-10:10 Break}

10:10

3aNSa5. Instrumentation calibration: The user/consultant's perspective. George Paul Wilson (Wilson, Ihrig \& Assoc., Inc., 5776 Broadway, Oakland, CA 94618)

Accurate and supportable measurements of noise levels require the user/consultant to frequently "calibrate" acoustical instrumentation, usually under "field" rather than laboratory conditions. Field use calibrators are subject to a variety of environmental and use conditions, but must maintain accuracy and have calibrations traceable to NIST standards. In many instances the measurement data are used as facts in litigation or to establish compliance with ordinances and applied standards. Other uses include comparison with measurement data by others, with previous data and with projections. A description of calibration and verification methods employed by consultants is presented along with an assessment of their effectiveness. Also, presented are comments on the need for education of users in the use and significance of instrument calibration and the requirement for frequent "traceable" confirmations of calibration. Of particular importance is the concept and use of a system calibration which includes all relevant instrumentation components.

10:30

3aNSa6. Instrument calibration: The hearing conservationist's perspective. Kevin L. Michael (Michael \& Assoc., Inc., 246 Woodland Dr., State College, PA 16803)

The audiometer is a critical element of the hearing conservationist's daily routine. The measurement of hearing thresholds with an instrument that is out of calibration can result in errors in permanent records, loss of work time due to retesting, and potential legal action. Subjective audiometer calibration must be part of the hearing conservationist's daily procedure. Calibration of the industrial audiometer must be performed at specified intervals at an appropriate laboratory. Factors related to the calibration procedure including cost, thoroughness, standards and the differences between calibration of industrial and clinical equipment are of inlerest to the hearing conservationist. Related issues including ambient room noise in the test booth, computer-based audiometry and the field measurement hearing protector attenuation must also be considered. 


\title{
Session 3aNSb
}

\section{Noise: Progress Report and Discussion on the Continuing Activity of ASA's Role in Noise and Its Control}

\author{
T. James DuBois, Chair \\ DuBois and Associates, 9424 Crystal View Drive, Tujunga, California 91042
}

Invited Paper

11:00

3aNSb1. Progress report and discussion on the continuing activity of ASA's role in noise and its control. T. James DuBois (DuBois and Assoc., 9424 Crystal View Dr., Tujunga, CA 91042)

A discussion meeting sponsored by the Technical Committee on Noise is held to review progress made to date on the actions initiated at the Denver meeting in 1993 to increase the role of the ASA in noise and noise control. Members of the steering committee and others involved in related supporting activity will review specific progress made in the areas of education, increasing public awareness about noise and noise control, and encouraging joint activity in this area with other professional organizations.

\section{Session 3aPAa}

\section{Physical Acoustics and Bioresponse to Vibration and to Ultrasound: Cavitation and Detection Monitoring}

\author{
Wesley L. Nyborg, Cochair \\ Physics Department, University of Vermont, Burlington, Vermont 05405 \\ Charles C. Church, Cochair \\ Molecular Biosystems, Inc., 10030 Bames Canyon Road, San Diego, Califormia 92121
}

Chair's Introduction-8:00

This special session deals with topics of bubble detection and cavitation monitoring in the ocean, in the laboratory and in man. The topics are relevant to sound propagation in the ocean, to industrial applications of ultrasound and to the safety and effectiveness of medical ultrasound. The title of the session is identical to that of a technical report under preparation by a working group (WG22) of the Accredited Standards Committee (SI) on Acoustics. In this introduction, the scope and purpose of the technical report will be explained, progress on its preparation will be described, and an invitation extended for suggestions to the working group.

\section{Invited Papers}

8:10

3aPAal. Measurements of passive and active microbubbles in the sea. Herman Medwin (Phys. Dept., Naval Postgrad. School, Monterey, CA 93943 and Ocean Acoust. Assoc., 4021 Sunridge Rd., Pebble Beach, CA 93953)

Around 1960 an oceanographer at the Navy Electronics Laboratory dared to question physics laboratory measurements when he wrote an internal memo titled “Do Invisible Microbubbles Exist at Sea?." Indeed they do! From 1964 to 1974 a series of M.S. students at the Naval Postgraduate School published the first microbubble density distributions at sea. These were measured by several in-situ ocean techniques including: change of transducer impedance; photography; excess attenuation, backscatter and sound-speed dispersion in a small pulse-echo system: Fourier transform of a sawtooth signal propagating between two separated hydrophones. An "unbelievable" immense number of coastal bubbles of radius 15 to $300 \mu \mathrm{m}$ were found. The 1990 s have seen a renaissance in the field. The spatial and temporal variation of bubble numbers have been studied, and acoustical oceanographers now use bubbles as tracers to determine ocean processes near the ocean surface. Sea state noise and rain noise have both been definitively ascribed to the radiation 
from huge numbers of infant microbubbles. Indeed, the "noises" have now become "signals." The underwater sound of breaking waves has been inverted to yield the spectrum of ocean wave height and recently the underwater sound of rainfall has been inverted to reveal the real-time rainfall drop size distribution. The distinctive spectrum of underwater sound during rainfall even allows one to describe the clouds from which the rain has fallen. [Work supported by ONR.]

\section{8:40}

3aPAa2. Acoustic detection of inertial microcavitation from ultrasound. Ronald A. Roy (Appl. Phys. Lab., Univ. of Washington, Seattle, WA 98195)

Recent years have witnessed a growing interest in processes goveming the nucleation, perpetuation, and physical consequences of inertial microcavitation generated by ultrasound. Fields as diverse as medicine, ultrasonic cleaning, and sonochemistry all rely, in varying degrees, on our ability to either inhibit, promote, or control inertial cavitation activity. Quantitative, spatially resolved assessment of such activity often requires an ability to detect bubbles as small as a micron lasting less than a microsecond. This constitutes a technical challenge that has sought the services of optical, electrical, and acoustical technologies. A review is presented of the state of the art of spatially resolved, acoustic microcavitation detection schemes. This will include descriptions of both passive [Atchley et al., Ultrasonics 26 (1988)] and active [Roy et al., J. Acoust. Soc. Am. 87, 2451 (1990)] systems. Although these systems were designed to detect inertial microcavitation generated by microsecond pulses of Megahertz-frequency ulerasound (similar to that utilized in diagnostic ultrasound), the techniques are quite straightforward and generalizable to any situation where one seeks to detect spatially discrete cavitation activity in a reasonably homogeneous bounded liquid. Issues such as absolute sensitivity and observational invasiveness are discussed. [Work supported by NIH through Grant No. ROl CA39374.]

9:10

3aPAa3. Detection of cavitation during clinical extracorporeal lithotripsy. Andrew J. Coleman, Min J. Choi, and John E. Saunders (Medical Phys. Directorate, Guy's and St. Thomas' Hospital Trust, London SEI 7EH, England)

A clinical cavitation detection system has been developed that monitors the amplitude-lime variation of the 1-MHz component of the broadband acoustic emission expected to accompany bubble collapse during clinical extracorporeal shockwave lithotripsy [C. C. Church, J. Acoust. Soc. Am. 86, 215-227 (1989)]. The detector is based on a hand-held piezoceramic focal bowl hydrophone that is acoustically coupled to the patients' skin. A commercial electromagnetic positioning device (Fastrak, Polhemus Inc., Vermont) is used to enable the reception zone of the hydrophone to be directed under ultrasound image guidance. The system has been tested in the clinic by monitoring acoustic emission from positions around the beam focus of a clinical shockwave lithotripter during routine lithotripsy. The detected acoustic emission shows features characteristic of those obtained from cavitation observed in vitro and arises from within a region of similar dimensions to that of the hyper-echoic region that can be simultaneously observed in the B-scan image. The study provides evidence that acoustic emission from cavitation in tissue can be detected noninvasively during clinical lithotripsy.

9:40

3aPAa4. Simultaneous bubble sizing using multiple acoustic techniques. T. G. Leighton (Inst. of Sound \& Vib. Res., Univ. of Southampton, Highfield, Southampton SO17 IBJ. England)

There exists a range of acoustic techniques for characterizing bubble populations within liquids. Each technique has limitations, and complete characterization of a population requires the simultaneous use of several, so that the limitations of each find compensation in the others. An experiment is described in which a simple controlled bubble population is subjected to a driving signal at pump frequency, $\omega_{p}$, and a second signal at the imaging frequency, $\omega_{i}$. The population is then simultaneous examined using geometrical scattering, and resonance scattering of the fundamental frequency $\left(\omega_{p}\right)$, second and third harmonics $\left(2 \omega_{p}, 3 \omega_{p}\right)$, and combination frequencies $\left(\omega_{1} \pm \omega_{p}\right.$ and $\left.\omega_{i} \pm \omega_{p} / 2\right)$. Comparison is made of the ease, accuracy, and speed with which individual techniques measure the population, and what advantages accrue from their simultaneous deployment.

10:10-10:25 Break

\section{Contributed Papers}

\section{0:25}

3aPAa5. Temperature-related effects in single-bubble sonoluminescence. Sean M. Cordry (York College, York, NE 68467), Lawrence A. Crum, and Ronald A. Roy (Appl. Phys. Lab., Seattle, WA 98105)

Bubbles acoustically levitated in the appropriate underwater sound field can undergo radial motion which causes the bubbles to be luminescent, a phenomenon known as single-bubble sonoluminescence (SBSL). It has been reported [Hiller et al., Phys. Rev. Lett. 69, 1182 (1992)] that cooling the temperature of the water by $20^{\circ} \mathrm{C}$ can increase light emission by a factor of 12.5. In this present study, the acoustic energy radiated by SBSL was measured using a needle hydrophone and the number of photons emitted by the bubble was measured with a photomultiplier tube. Comparisons were made between the acoustic and electromagnetic radiation emitted by the bubble for a range of different temperatures and acous- tic pressures. Although the number of photons emitted varied strongly with the temperature, the radiated acoustic energy did not. These results suggest that it is the internal gas dynamics and not the bubble dynamics which is responsible for the observed temperature-related effects. [Work supported by the Office of Naval Research.]

\section{0:40}

3aPAa6. Correlation of cavitation-induced damage to blood elements with passive acoustic detector output. E. Carr Everbach, Inder Raj S. Makin (Eng. Dept., Swarthmore College, 500 College Ave., Swarthmore, PA 19081), and Charles W. Francis (Strong Memorial Hospital, Univ. of Rochester, Rochester, NY 14642)

A 20-MHz probe transducer placed confocally with a 1-MHz cavitation transducer was used to detect inertial cavitation in human blood preparations [Huertas et al., J. Acoust. Soc. Am. 95, 2856(A) (1994)]. The prepa- 
rations included suspensions of red blood cells (RBCs) and platelets in autologous plasma with or without the addition of Albunex (R) microbubble contrast agent. Damage to RBCs and platelets was measured hematologically and correlated with statistical descriptors of the fluctuating voltages from the passive cavitation detector. Good correlations were obtained between the average rms cavitation signal and percent hemolysis of diluted RBCs. In the platelet preparations, detector output paralleled damage as measured both by Coulter counter and by leakage of radiolabeled chromium-51 through platelet membranes. Advantages and shortcomings of the passive acoustic detection scheme used to predict cavitational damage to blood constituents will be discussed. [Work supported by an NSF PFF.]

\section{0:55}

3aPAa7. Direct observation of microbubble oscillations. Yuren Tian, Daniel Riegel, Jeff Ketterling, and Robert E. Apfel (Ctr. for Ultrasonics and Sonics, Yale Univ., New Haven, CT 06520-8286)

The study of microbubble oscillations is important for the understanding of sonoluminescence phenomena. Bubble dynamic behavior is conventionally detected with light scattering techniques. This method generally gives little information about the shapes of a bubble during its oscillation. In order to observe the bubble shapes, a direct imaging system was set up. The shape of a bubble levitated in a liquid is magnified and displayed on a TV screen. The bubble is illuminated with an LED lamp which is strobed at a frequency slightly different from the driving sound field. This technique can slow the moving image of the bubble, allowing one to observe the shape of a bubble oscillating between 5 to $100 \mu \mathrm{m}$ in diameter. Experiments show that when a bubble oscillates with sonoluminescence, it keeps a closely spherical shape during the entire oscillation period. However, with the increase of gas concentration in the host liquid, sonoluminescence disappears and higher mode shapes are developed during the bubble's shrinking period. Experiments also show that the asymmetric shape of the bubble may cause the levitated bubble to become unstable. [Work supported by NASA through JPL, contract 958722.]

\section{1:10}

3aPAa8. Pulse width measurements of sonoluminescence in cavitation-bubble fields. Thomas J. Matula, Ronald A. Roy, and Pierre D. Mourad (Appl. Phys. Lab., Univ. of Washington, Seattle, WA 98105)

Recent studies involving sonoluminescence have focused on the properties and behavior of single bubbles in isolation. Some properties of single-bubble sonoluminescence (SBSL) appear to conflict with wellknown properties of sonoluminescence from cavitation fields (MBSL). Direct comparisons between MBSL and SBSL have been difficult due to the difficulty in generating SBSL in nonaqueous solutions. A recent study comparing MBSL and SBSL [T. J. Matula et al., J. Acoust. Soc. Am. 96, 3252(A) (1994)] has shown that aqueous solutions of nonvolatile solutes show distinct differences in the corresponding spectra. The differences are attributed to differences in the relative symmetry of collapse of bubbles in cavitation fields versus in isolation. As part of the ongoing study to compare MBSL and SBSL, measurements were made of the apparent flash width of sonoluminescence from cavitation tields. Various well-defined cavitation regions were studied and compared to SBSL flash widths using an identical PMT for both cases. Both invasive and noninvasive mechanisms for generating MBSL were studied to determine the relative influence of ultrasonic horn immersion. Progress toward identifying the similarities and dissimilarities between MBSL and SBSL should lead to an improved understanding of sonochemical reactions. [Work supported by ONR.]
3aPAa9. Traveling wave impulses in synchronous sonoluminescence. Fred B. Seeley (Dept. of Phys., Univ. of Alabama, Huntsville, AL 35899) and Jonathan P. Dowling (U.S. Army Missile Command, Redstone Arsenal, AL 35898-5248)

Observations and measurements are reported of trains of travelingwave pressure impulses that are coincident with the onset of stable, synchronous sonoluminescence. Each pressure impulse is initiated by a hard collapse of the centered bubble, travels outward to the flask boundary, is reflected, and then reconverges. The converging impulse apparently rebounds from the free boundary of the bubble and the impulse continues traveling outward and inward until it is synchronized with a subsequent collapse. It is hypothesized that synchronous sonoluminescence is produced when this train of spherically symmetric, concentric pressure traveling impulses are synchronized with the driving standing wave. Experimental observations of integral numbers of such impulses for a number of spherical flasks at several harmonics support this hypothesis. Theoretical analyses of these experimental observations and measurements will be presented.

\section{1:40}

3aPAa10. Electrical matching of power transducers for acoustic cavitation applications. Isabelle Looten, Jamal Assaad, and Jacques Frohly (Dept. of OAE, IEMN, Valenciennes Univ., BP 311 Le Mont-Houy, 59304 Valenciennes, France)

Over the last 20 years, a large number of studies have been devoted to acoustic cavitation in liquids. High power transducers are needed to generate cavitation. A transducer working at $500 \mathrm{kHz}$ will be presented. This transducer has been electrically matched using a stainless steel layer welded to a piezoelectric element. Optimal thickness of the matching layer, which is considered as a transmission line, has been found using a Mason's circuit. The transducer conversion efficiency is experimentally evaluated to determine the acoustic pressure amplitude needed to generate cavitation in a liquid. The cavitation noise recorded with a hydrophone is used to characterize the cavitation state in the fluid. The energy of this noise is correlated to the acoustic pressure amplitude. Then the correlation between cavitation noise energy and chemical reactivity in homogeneous chemistry is also made. The bio-effect of cavitation on E. Coli cells is presented as a function of cavitation noise energy. It is evidenced that cavitation noise energy is an efficient tool to characterize a cavitation state in a fluid and to predict its chemical and bacteriological effects.

\section{1:55}

3aPAal1. Measurements of ultrasonic pulse distortion produced by the chest wall. Laura M. Hinkelman, Robert C. Waag (Dept. of Elec. Eng., Univ. of Rochester, Rochester, NY 14627), and Thomas L. Szabo (Hewlett-Packard Imaging Systems, Andover, MA 01810)

Ultrasonic wavefront distortion produced by transmission through human chest wall specimens was measured. Pulses with a center frequency of $2.3 \mathrm{MHz}$ were received over a $20.00 \times 20.16 \mathrm{~mm}^{2}$ area with a measurement spot size of $0.21 \times 0.40 \mathrm{~mm}^{2}$ after propagation through a chest wall specimen. Secondary wavefronts produced by interactions between transmitted pulses and structures of the rib cage sometimes disrupted the main wavefront and interfered with the determination of wavefront distortion caused by soft tissue inhomogeneities. Differences in arrival time and energy level between $11.60 \times 14.28 \mathrm{~mm}^{2}$ regions of the measured waveforms that did not include secondary wavefronts and references that account for geometric delay and spreading were computed. For 16 different intercostal spaces, the average rms value of the arrival time fluctuations was $21.3 \mathrm{~ns}$ with a mean correlation length of $2.50 \mathrm{~mm}$. The energy level fluctuations had an average ms value of $1.57 \mathrm{~dB}$ and a correlation length of $1.98 \mathrm{~mm}$. The results indicate that soft tissue inhomogeneities in chest wall specimens significantly distort ultrasonic pulses in the low $\mathrm{MHz}$ frequency range, although the magnitude of this distortion is noticeably smaller than that measured for abdominal wall specimens at $3.75 \mathrm{MHz}$ [Hinkelman et al., J. Acoust. Soc. Am. 95, 530-541 (1994)]. 


\title{
Session 3aPAb
}

\section{Physical Acoustics: Outdoor Sound Propagation}

\author{
Richard Raspet, Chair \\ Department of Physics and Astronomy, University of Mississippi, University, Mississippi 38677
}

\section{Contributed Papers}

\section{8:00}

3aPAb1. Initial measurements of sound propagation through turbulence in a wind tunnel. Randall W. Smith and Russell $E$. Henrichs (Appl. Res. Labs., Univ. of Texas, 10,000 Burnet Rd., Austin, TX 78713)

Outdoor experiments of sound propagation through atmospheric turbulence are complicated by the difficulty of making sufficient measurements to fully characterize the flow field. Therefore the authors have initiated a series of experiments in a wind tunnel. The wind tunnel provides a means of creating various inhomogeneous flow fields through which to propagate sound. These wind tunnel flows are more easily measured and characterized than the atmospheric boundary layer. The goal of the experiments is to examine in detail the propagation of sound through increasingly complex flow fields, and to understand the effect of large-scale coherent turbulent motions on sound propagation. This presentation provides an overview of the experimental setup, a discussion of scaling issues, and preliminary analysis of data from the initial experiments. Some of the lessons learned from these initial experiments also will be discussed.

\section{8:15}

3aPAb2. Influence of turbulent intermittency on acoustic scattering in the atmosphere. D. Keith Wilson (Dept. of Meteorol., Penn State Univ., 503 Walker Bldg., University Park, PA 16801)

The classical treatment of wave scattering by turbulence does not account for the influence of turbulent intermittency. Intermittency causes quantities such as the scattering cross section to vary in space and time. It is particularly important to account for intermittency when there are energetic eddies having dimensions larger than the scattering volume, as is typically the case for scattering problems involving atmospheric turbulence. The conditional-probability method developed by Gurvich and Kukharets for incorporating intermittency effects will be discussed and applied to the problem of acoustic wave scattering by atmospheric turbulence. It will be shown that intermittency can dramatically increase the probability of measuring large values of the scattered intensity. The effect of combined intermittency in the temperature and velocity fields will also be considered.

8:30

3aPAb3. Experimental observation of the effects of turbulence intermittency on scattered sound. David I. Havelock (Inst. for Microstructural Sciences, Natl. Res. Council, Ottawa, ON KIA 0R6, Canada)

Fully developed turbulence follows the well-known Kolmogorov spectrum and, within the inertial subrange, is governed by a single parameter $\epsilon$ called the viscous dissipation rate. Under stationary conditions the intensity of sound scattered from turbulence follows an exponential distribution with mean intensity $I_{0}$ determined by $\epsilon$. In a more realistic turbulence model, the viscous dissipation rate for a given scattering volume fluctuates with a log-normal distribution. The corresponding fluctuations in $I_{0}$ cause the intensity distribution to deviate from the exponential distribution. In particular, the tail of the distribution is raised, providing more frequent occurrences of higher intensity levels. This effect impacts on target detection probability in acoustic remote sensing applications. It is shown that the deviations from an exponential distribution are clearly observable in direct measurements of sound intensities within a refractive shadow near the ground. The variance $\sigma$ of the fluctuations in the dissipation rate is estimated by comparing the measured and theoretical distributions. It is also shown that $\sigma$ cannot be obtained directly from short-term estimates of $I_{0}$.

\section{$8: 45$}

3aPAb4. On the turbulence-induced thickening of sonic boom shock waves. Kenneth J. Plotkin (Wyle Labs., 2001 Jefferson Davis Hwy., Ste. 701, Arlington, VA 22202)

Two well-accepted effects of turbulence on sonic boom shock waves are the presence of random perturbations behind the shock waves, and random thickening of ths shocks themselves. Crow [J. Fluid Mech. 37, 529-563 (1969)] proposed a first-scattering model for the perturbations. This theory has been well accepted. Plotkin and George [J. Fluid Mech. 54, 449-467 (1972)] proposed a second-scattering/energy-balance theory for shock thickening. Pierce [J. Acoust. Soc. Am. 49, 906-924 (1971)] proposed a shock folding theory for thickening, and has recently [AIAA Paper 95-105] proposed a dispersion model. These thickening models present different perspectives, but are consistent with each other. Small-scale experiments by several workers have failed to fully replicate shock thickening, with consequent assertions that either thickening does not occur, or that it does occur but that the various theories are incorrect. It has been found that those experiments do not fully replicate the fight-test conditions, and that formulas from the theories have been applied without testing whether the necessary conditions have been met. A variation of the Plotkin/George theory is presented, which more closely relates that theory to Crow's perturbation model.

\section{9:00}

3aPAb5. Omega-to-the-one-third term in dispersion relation for acoustic pulse propagation through turbulence. Allan D. Pierce (Boston Univ., Dept. of Aerospace and Mech. Eng., 110 Cummington St., Boston, MA 02215)

The author's earlier formulation of pulse propagation through turbulence required a somewhat $a d$ hoc separation of the effects of large scale and small scale turbulence with the selection of a cut-off turbulent wave number $k_{c}$ that separates the two regimes. A neater-cleaner formulation proceeds with the premise that the frequency dispersion of pulses is caused by that part of the turbulence spectrum which lies in the inertial range originally predicted by Kolmogoroff. The acoustic propagating wave's dispersion relation has the acoustic wave number being of the form $k=(\omega / c)+F(\omega)$, where $c$ is a spatially averaged sound speed and where, for mechanical turbulence, the extra term $F(\omega)$ must depend on only the angular frequency $\omega$, the sound speed $c$, and the turbulent energy dissipation $\epsilon$ per unit fluid mass and per unit time. If the turbulence is weak, then the quantity $F(\omega)$ has to be of second order in the portions of the turbulent fluid velocity in the intertial range, so, following Kolmogoroff's reasoning, it must vary with $\epsilon$ as $\epsilon^{2 / 3}$. Simple dimensional analysis then 
reveals that $F(\omega)$ is $K \epsilon^{2 / 3} c^{-7 / 3} \omega^{1 / 3}$, the latter factor being as announced in the title of this abstract, and $K$ being a universal dimensionless complex constant. A similar result holds for thermal turbulence. The analysis showing that the separating-out of the effects of turbulence in the inertial regime is in fact possible yields $K=-0.37 e^{i \pi / 3}$. The dispersion is typically small, but has an accumulative effect that leads to a sizable pulse distortion over large propagation distances. [Work supported by NASA Langley Research Center.]

\section{9:15}

3aPAb6. New formulations for the scattering of sound in a moving random medium. John M. Noble (U. S. Army Res. Lab., Battlefield Environment Directorate, ATTN: AMSRL-BE-S, White Sands Missile Range, NM 88002) and V. E. Ostashev (Fachbereich Physik, Universitat Oldenburg, Oldenburg, Germany)

The main aim of the presentation is the review of the modern theory of sound propagation and scattering in media with random inhomogeneities of the adiabatic sound speed, density, and medium velocity (mainly, in the atmosphere and ocean). This theory has been intensively developed in the last few years and includes the rigorous calculations of sound field statistical moments using Bom approximation, ray, Rytov, and parabolic equation methods, and the theory of multiple scattering. This modern theory also shows that certain equations for statistical moments of the sound field propagating in the turbulent atmosphere, which previously have been widely used in the literature, must be revised. On the basis of the theory developed, new possible methods for acoustic remote sensing of the atmosphere are proposed.

\section{9:30}

3aPAb7. On the influence of turbulence modeling for atmospheric sound propagation. Ph. Blanc-Benon, D. Juvé, and P. Chevret (Lab. de Mécanique des Fluides et d'Acoustique, URA CNRS 263, Ecole Centrale de Lyon, BP 163, 69131 Ecully Cedex, France)

Incorporating random aspects in the numerical simulation of atmospheric sound propagation has led to a much better agreement between experiments and predictions. In particular, the turbulent scattering of sound into acoustic shadows had been demonstrated but some discrepancies still exist. In most of the numerical studies the fluctuations of the refractive index have been considered as scalar and characterized by a single length scale (Gaussian spectrum). However, sound propagation in the turbulent atmosphere is affected by quantities with different tensorial character and different scales. In this paper two possible ways of improving the simulations are investigated: by choosing a better spectral representation of the turbulence (von Karman instead of Gaussian form); by correctly taking into account the vectorial character of wind fluctuations. The turbulence is represented as a set of realizations of a random field generated by a limited number of scalar or vectorial random Fourier modes. Through each individual realization, the acoustic pressure field is computed with a wideangle parabolic approximation. Ensemble averaging is then performed to obtain the statistical properties of the acoustic field: mean level, standard deviation of the fluctuations. Illustrations will be given for an upward refracting atmosphere, when a deep deterministic shadow zone is present.

\section{9:45-10:00 Break}

\section{0:00}

3aPAb8. Wideband sound propagation in a refractive shadow zone: Tests of turbulence models. Xiao Di, Kenneth E. Gilbert (Appl. Res. Lab. and the Graduate Prog. in Acoust., Penn State Univ., P.O. Box 30, State College, PA 16804), David I. Havelock, Michael R. Stinson, and Gilles A. Daigle (Inst. for Microstructural Sci., Natl. Res. Council, Ottawa, ON KIA 0R6, Canada)

A wideband sound propagation experiment using discrete tones from 40 to $940 \mathrm{~Hz}$ has been conducted and analyzed to determine soundpressure levels in a refractive shadow zone. It was found that at $\mathbf{4 0}$ and $\mathbf{9 0}$ $\mathrm{Hz}$, the effect of turbulence was negligible. At $210 \mathrm{~Hz}$ and above, turbu- lence effects were significant. From 380 to $940 \mathrm{~Hz}$, the shadow zone levels showed a relatively weak dependence on frequency. To test two turbulence models (Gaussian and Kolmogorov), the experimentally observed levels in the shadow zone were compared with parabolic equation calculations. The parameters of the turbulence models were determined from meteorological measurements. The Gaussian model, which agreed with acoustic data only over a fairly narrow range of frequencies, gave a strong frequency dependence for the levels in the shadow zone. The Kolmogorov model, on the other hand, gave good agreement with experiment over the entire band of frequencies, showing a relatively weak dependence on frequency from 380 to $940 \mathrm{~Hz}$. It was concluded that the Kolmogorov turbulence model, which is consistent with inertial subrange turbulence, is a valid model for outdoor sound propagation predictions over a wide frequency range. The Gaussian model, which overpredicts the frequency dependence, is valid only in a narrow range of frequencies. [Work partially supported by the Army Research Laboratory.]

\section{0:15}

3aPAb9. Importance of the near-ground sound-speed profile in prediction of sound fields in a refiractive shadow. Michael $R$. Stinson (Inst. for Microstructural Sciences, Natl. Res. Council, Ottawa, ON K1A 0R6, Canada)

The influence of the vertical sound-speed profile on sound propagation in an acoustic shadow has been investigated. For upwardly refracting conditions with both source and receiver near the ground, the profile in the first couple of meters nearest the ground can be critically important in determining the sound-pressure level in the acoustic shadow. A meteorological tower has been used to determine sound-speed profiles, in conjunction with acoustical propagation experiments. Wind speed and direction measured at a 10-m height and temperatures measured at heights of 8.7 and $2 \mathrm{~m}$ are used with similarity scaling expressions [L'Espérance et al., Appl. Acoust. 40, 325-346 (1993)] to obtain predicted vertical profiles for wind speed, temperature, and sound speed. These predicted profiles can be tested using additional measurements of wind speed and temperature at other heights. It is found that the similarity-based profiles do not always describe the actual profiles close to the ground. The implications for the prediction of sound fields in an acoustic shadow are investigated numerically using a fast, parabolic equation approach that includes profile, atmospheric turbulence, and ground impedance effects.

\section{0:30}

3aPAb10. Efficient computation of sound field in horizontally stratified media using a WKB-type approximation with Airy functions. Y. L. Li (Dept. of Elec. and Comput. Eng., Univ. of Illinois, Rm. 332 North CSRL, 1308 W. Main St., Urbana, Illinois 61801)

Conventional fast field programs model a general refractive index profile with a system of horizontal layers. The choice of layer thickness and number of layers depends on frequency, range, and intrinsic properties of the refractive index profile. These constraints result in excessive computation time for many practical cases. To avoid the long run times associated with the layered model, a WKB-type approximation with Airy functions has been applied to the efficient computation of the height-dependent acoustic pressure. Numerical results demonstrate that the new fast field program can reduce the computer time about 50 -fold. For higher frequencies, the new fast field program can save more computer time. [Work supported by the US Army CERL.]

\section{0:45}

3aPAb11. An improved approximation for sound propagation in a medium with a linear sound-speed profile. Michael J. White (U.S. Army Construction Eng. Res. Lab., P.O. Box 9005, Champaign, IL 61826-9005), Y. L. Li (Univ. of Illinois, Urbana, IL 61801), and Jianfeng Tai (U.S. Army Construction Eng. Res. Lab., Champaign, IL 61826-9005)

The use of a refractive index profile whose square varies linearly with height is a common approximation when solving for the sound propagation in a refracting atmosphere. Its solutions are more tractable than those for an atmosphere whose sound speed varies linearly with height, although the 
linear sound-speed profile is more often the one whose solution is sought In this paper, the approximate relationship between the two profiles has been examined. Numerical examples show that the two profiles give similar solutions for the sound pressure only when the background atmosphere is upward refracting. When the atmosphere is downward refracting, however, the two profiles give results that disagree except when the refraction is extremely small. In this paper, an improved approximate solution for the pressure field is given for the linear sound-speed profile. The solution is based on a WKB-type approximation which uses Airy functions as the general solutions of the wave equation. Numerical comparisons are made to show that the new approximation adequately models the sound field for the linear sound-speed profile for a wide range of detector heights and gradients and for both upward and downward refracting atmospheres.

\section{1:00}

3aPAb12. Further investigations on the propagation of sound over flat rough finite impedance surfaces. James $P$. Chambers and James M. Sabatier (Natl. Ctr. for Physical Acoust., Univ. of Mississippi, University, MS 38677)

The propagation of sound over rough surfaces of finite impedance is investigated. Initial experiments have illustrated the excess attenuation caused by roughness, that is, attenuation above and beyond that caused by geometrical spreading alone. These initial experiments were conducted indoors in a well-controlled setting for sound at grazing incidence. These measurements also show good agreement with Attenborough's proposed model [J. Acoust. Soc. Am. 95(5), 2838(A) (1994)] that incorporates the effects of roughness into an effective admittance. The purpose of this present work is to find out if these trends persist in the propagation of sound over rough surfaces in a real-world setting. Sound signals will be measured above both rough and smooth agricultural surfaces with varying roughness profiles. Additional measurements will be made to determine the impedance and wave number of the surface as well as the roughness profile in order to examine Attenborough's model in more detail. [Work supported by USDA.]

\section{1:15}

3aPAb13. Tank field data compared with fast field program. Dean Klein (Dept. of Mech. Eng., New Mexico State Univ., Las Cruces, NM 88003) and John M. Noble (U. S. Army Res. Lab., Battlefield Environment Directorate, ATTN: AMSRL-BE-S, White Sands Missile Range, NM 88002)

Acoustic data were recorded from various tanks traveling a known path at constant speed. The data were compared with the fast field program (FFP) to determine how well the FFP model would collate with actual data covering a range up to $2 \mathrm{~km}$. This was done to determine if the FFP could be used to reliably predict the propagation effects of the environment for acoustical arrays listening for tanks. As the tanks were run through their course, meteorological data were also recorded. The meteorological data included temperature, relative humidity, barometric pressure, wind speed, and wind direction from the surface to $2000 \mathrm{~m}$. The comparisons were encouraging through most cases until tank transmissions were buried in the background noise.

\title{
Session 3aPP
}

\section{Psychological and Physiological Acoustics: Pitch, Loudness and Complex Sound Processing}

\author{
Daniel L. Weber, Chair \\ Department of Psychology, Wright State University, Dayton, Ohio 45435
}

\section{Contributed Papers}

\section{$8: 30$}

3aPP1. Information integration of frequency and duration. Robert D. Irwin and Daniel L. Weber (Dept. of Psych., Wright State Univ., Dayton, OH 45435)

The distribution discrimination procedure can be used to evaluate a listener's ability to discriminate along a single dimension of an auditory signal. This can be accomplished by sampling from two different distributions of the parameter and asking the listener to identify which sample was most likely to have come from the distribution with the higher mean. Information integration can be measured as the improvement in performance when multiple samples are presented. Whether information integration appears to be different for frequency discrimination and duration discrimination was examined. Subjects discriminated duration or frequency in a 2IFC task when different numbers of samples were presented. In the discrimination of the duration of a $1-\mathrm{kHz}$ sinusoid, samples were drawn from normal distributions with either a mean of 100 or $130 \mathrm{~ms}$; both had a variance of $900 \mathrm{~ms}$. In the discrimination of the frequency of a $100-\mathrm{ms}$ sinusoid, samples were drawn from normal distributions with means of 565 and $570 \mathrm{~Hz}$ and a variance of $25 \mathrm{~Hz}$ and from distributions with means of 565 and $585 \mathrm{~Hz}$ and a variance of $400 \mathrm{~Hz}$. These conditions should be alike to an ideal observer in that all involve a standard deviation equal to the difference between their means. The majority of the listeners show better information integration for signal duration than signal frequency. [Work supported by AFOSR through WPAFB AL/CFBA.]

\section{8:45}

3aPP2. Processing of $\log$ and linear ripple spectra. William A. Yost (Parmly Hearing Inst., Loyola Univ. Chicago, 6525 N. Sheridan Rd., Chicago, IL 60626)

Two sets of experiments were conducted involving spectra with sinusoidal ripples of amplitude versus frequency. For one set of stimuli the sinusoidal spectral ripple was on a linear frequency scale while for the other set the ripple was on a $\log$ frequency scale. For both sets of stimuli ripple frequency and ripple phase were varied. In experiment I, listeners were asked to determine if a change in ripple stimuli was a ripple frequency change or a ripple phase change. They were also asked to differentiate between log and linear ripples. Except for a few conditions, listeners were unable to differentiate between linear and log ripples and between spatial frequency and spatial phase changes. In experiment II, listeners were asked to discriminate between ripple spectra and flat spectra. The ripple spectral depth required for discrimination was approximately the same for both log and linear ripples. The results will be discussed in terms 
of suggestions that the auditory system performs a type of spatial frequency analysis similar to that performed by the visual system. [Work supported by NIDCD and AFOSR.]

\section{9:00}

3aPP3. Determinants of the perceptual similarity of complex filter shapes. Yijian Zheng and Charles S. Watson (Dept. of Speech and Hearing Sciences, Indiana Univ., Bloomington, IN 47405)

Sounds were created by passing an increasing-frequency 300-ms sawtooth $(120-170 \mathrm{~Hz})$ through each of the 15 complex filters. The filters were created by varying two parameters of a pair of overlapping secondorder filters $(C F: 500$ and $1500 \mathrm{~Hz})$. The parameters varied were the width of the upper filter $(Q: 1,3,8)$ and the relative amplitudes of the two filter peaks $(+12,+6,0,-6$, or $-12 \mathrm{~dB})$. Listeners judged the similarity of pairs of these sounds, equated for energy, using a ten-point scale. A multidimensional scaling (MDS) analysis suggested that when $Q$ of the upper filter was low, the sounds were distinguished entirely on the basis of the relative amplitudes of the filters. For high upper-filter $Q$ values, a different intensity-related dimension was the basis for distinguishing among the sounds, which were essentially identical on dimension No. 1 . The two dimensions are associated with overall pitch, and with the salience of the high-frequency spectral peak. Various physical models are fitted to the data. [Work supported by NIDCD and by AFOSR.]

\section{9:15}

3aPP4. Diufhuis pitch and other anomaly pitches. Jian-Yu Lin and William Morris Hartmann (Dept. of Phys., Michigan State Univ., East Lansing, MI 48824)

"Anomaly pitches" are tonal sensations caused by anomalies in the spectrum of a low-frequency periodic wave, for example, by a missing harmonic [Duifhuis, J. Acoust. Soc. Am. 48, 888 (1970)]- They are immediately heard in the steady state and do not require any reference signal. This paper reports anomaly pitches for both missing harmonics and inverted harmonics. They occur in pulse waves (for any constant phase), in sawtooth waves, in waves with alternating sine and cosine phases, and in waves with Schroeder phase. With a series of missing upper harmonics it is possible to make a virtual anomaly pitch with a missing fundamental. The observations can be compared with the original explanation of the effect by Duifhuis based upon detection of the anomalous harmonic during gaps. Some of the conditions press this explanation to the limit but none has proved fatal. [Research supported by the NIDCD of the NIH.]

\section{9:30}

3aPP5. Pitch ringing induced by frequency-modulated tones. Kiyoaki Aikawa, Minoru Tsuzaki, Hideki Kawahara, and Yoh'ichi Tohkura (ATR Human Information Processing Res. Labs., 2-2 Hikaridai, Seikacho, Sorakugun, Kyoto 619-02, Japan)

It was discovered that specific FM (frequency-modulated) tones induce a ringing of a perceived pitch. A mathematical model was derived to explain this phenomenon. An abrupt change of the slope in a unidirectional FM tone induces ringing of the perceived pitch. The typical ring-inducing FM tone had a piecewise linear frequency trajectory in the log frequency axis with three parts: (1) frequency onset at $1 \mathrm{kHz}$ rising to $0.732 \mathrm{kHz}$ in $200 \mathrm{~ms}$; (2) constant frequency at $1.732 \mathrm{kHz}$ for $200 \mathrm{~ms}$; and (3) frequency rising to $3 \mathrm{kHz}$ in $200 \mathrm{~ms}$. Several listeners reported one to three ringings around the middle part of the piecewise linear sweep. Frequent repetition of the listening test decreased the sensitivity of ringing perception. The ringing phenomenon can be explained by a second-order system as a functional model of sweep tracking. In order to provide experimental evidence for the second-order tracking system, specific values for the natural frequency and the damping factor were estimated. An inverse filter of this system was then constructed and was called the antiringing filter. In a psychophysical experiment, subjects compared the original piecewise sweep tone to that tone processed by the antiringing filter. Results demonstrated that pitch ringing was significantly suppressed by the antiringing filter.

\section{9:45-10:00 Break}

\section{0:00}

3aPP6. Auditory backward recognition masking: Influence of musical meaning on pitch relationship between first and second sounds. Yuki Kakita and Takahasi Hamada (Dept. of System Design Eng., Kanazawa Inst. of Technol., 7-1, Ohgigaoka, Nonoichi-machi, Kanazawa-Minami 921, Japan)

Auditory backward recognition masking (ABRM) refers to the interference of a second sound on recognition of another sound presented earlier in time. The influence of musical meaning on recognition of the first sound was investigated using the stimuli where the pitch relationship between the first and second sounds composed a triad. Four complex tones consisting of three tones differing in pitch were presented as stimuli. The frequency relationship of two complex tones composed a triad. The others did not compose a triad. One of the three tones of the complex tones was employed as a first sound. The others were employed as a second sound. The task was to label the stimuli as one of the four complex tones. The effect of the differences in the stimuli was evaluated using the percentage of correct responses as a function of ISI. Backward recognition masking was observed with the two sounds that did not compose a triad. The performance was better when the two sounds composed a triad rather than when they did not compose a triad. The results indicated that interference of a second sound on recognition of a first sound is reduced when there is musical meaning in the pitch relationship between the first and second tones.

\section{0:15}

3aPP7. A neural model of auditory scene analysis and source segregation. Stephen Grossberg (Cognitive and Neural Systems Dept., Boston Univ., 111 Cummington St., Rm. 244, Boston, MA 02215), Krishna K. Govindarajan (Res. Lab. of Electron., MIT), Lonce L Wyse (Inst. for Systems Sci., Natl. Univ. of Singapore), and Michael A Cohen (Cognitive and Neural Systems Dept, Boston Univ.)

In environments with multiple sound sources, the auditory system is capable of teasing apart the impinging jumbled signal into different mental objects or streams. A neural model of auditory scene analysis is presented that groups different frequency components based on pitch and spatial location cues, and selectively allocates the components to different streams. Grouping is accomplished through a resonance that develops between a given object's pitch, its harmonic spectral components, and (to a lesser extent) its spatial location. Those spectral components that are not reinforced by being matched with the top-down prototype read-out by the selected object's pitch representation are suppressed, thereby allowing another stream to capture these components. The model simulates data from psychophysical grouping experiments, such as how an upward sweeping tone creates a bounce percept by grouping with a downward sweeping tone due to frequency proximity, even if noise replaces the tones at their intersection point. The model also simulates illusory auditory percepts such as the auditory continuity illusion, and the Deutsch scale illusion whereby downward and upward scales presented alternately to two ears regroup based on frequency proximity. Stream resonances provide the coherence whereby one voice or instrument is tracked through a multiple source environment.

\section{0:30}

3aPP8. Efiects of signal frequency and ear of input on ICP-determined loudness adaptation. Robert S. Tannen, Emest M. Weiler, Joel S. Warm, and William N. Dember (Psychoacoustics Lab., Univ. of Cincinnati, Cincinnati, OH 45221)

The ipsilateral comparison paradigm (ICP) uses a monaurally presented base tone punctuated by referent tones of greater or less intensity to reveal magnitude-estimated loudness adaptation. Research with this technique has demonstrated adaptation across a broad spectrum of base-tone intensities [Dange et al., J. Gen. Psychol. 120, 217-274 (1993)]. Given evidence for different coding mechanisms at high and low signal frequen- 
cies and ear differences in adaptation [Ivry and Libby, Psychol. Sci. 4, 41-45 (1993); Davis and Weiler, Br. J. Audiol. 12, 59-60 (1978)], the present study examined the generality of ICP-determined loudness adaptation for the two ears at frequencies of 250,1000 , and $4000 \mathrm{~Hz}$. Base tone intensity in all conditions was $50 \mathrm{dBA}$. Three referent conditions $(+10 \mathrm{~dB}$ referent, $-10 \mathrm{~dB}$ referent, and a no referent control) were also employed. The generality of ICP-determined adaptation was demonstrated by similar declines in loudness over time in all ear/frequency combinations when referent tones were present. Adaptation effects were noted for the base and for the referent tones. In addition, adaptation was stronger for the base tone in comparison to the $+10 \mathrm{~dB}$ referent and for the $-10 \mathrm{~dB}$ referent in comparison to the base, a result consistent with Hood's model of loudness adaptation [Weiler and Hood, Audiology 16, 499-506 (1977)].

\section{0:45}

3aPP9. Loudness-data basis for "FIG6" hearing-aid fitting targets. Mead C. Killion (Etymotic Res., 61 Martin Lane, Elk Grove Village, IL 60007)

Loudness data from Pascoe (1988), Hellman et al. (1991), Lippman (1981) and Lyregaard (1988) provide the basis for a hearing-thresholdbased set of three frequency-response fitting targets: one each for 40,65 , and $90 \mathrm{~dB}$ SPL inputs. Although individual loudness measurements obtained using either the automated LGOB or LGCM loudness-growth tests generally agree with average data, Niemeyer's (1971) report that subjects with prolonged exposure to intense noise may have loudness discomfort levels elevated by $30 \mathrm{~dB}$ or more suggests caution in applying FIG6 to someone who has been wearing powerful linear hearing aids.

\section{1:00}

3aPP10. Control strategies for neurons modeled by self-exciting point processes. Laurence S. Irlicht and Graeme M. Clark (Australian Bionic Ear and Hearing Res. Inst., 384-388 Albert Rd., E. Melbourne, Victoria 3002, Australia)

Experiments demonstrate that electric stimulation of the auditory nerve evokes responses, which are qualitatively different to those of normal hearing. A self-exciting point process model of the neural response is applied to explain and predict responses for both situations. For acoustic stimulation, the model predicts the initial responses to a tone, average firing rate, maximum firing rate, and the shape of the per-stimulus time histogram. The model also predicts the neural response to pulsatile electric stimulation. For applications such as cochlear implants, it would be beneficial if the acoustic and electric responses were more similar. The model is used to parametrize the response differences in each case and based on these parameters to design electrical stimuli, which cause a neuron to respond similarly to the normal hearing situation. Such stimuli should find application in advanced cochlear implants.

\title{
Session 3aSA
}

\section{Structural Acoustics and Vibration: Radiation and Scattering from Shells}

\author{
Courtney B. Burroughs, Chair \\ Applied Research Laboratory, Pennsylvania State University, P.O. Box 30, State College, Pennsylvania 16804
}

\section{Contributed Papers}

\section{8:00}

3aSA1. Benchmarking the Kirchhoff approximation for specular scatter from a finite length cylindrical scatterer. Kevin D. LePage (Bolt Beranek and Newman Inc., 70 Fawcett St., Cambridge, MA 02138)

Recent work at BBN has been directed toward understanding and parametrizing the low- to mid-frequency, broadband scattered signature of submarines in terms of a small set of physical mechanisms. These mechanisms include parametrized dispersion curves, end-cap reflection coefficients, and end-cap radiation and excitation coefficients. Here, the Kirchhoff approximation is applied to model the early time portion of the backscattered signal. Two issues present themselves with this approach: (1) What is the correct frequency-dependent background impedance of the hull; and (2) for what frequencies and range of bistatic angles does the Kirchhoff approximation provide adequate performance. Here, the later issue is addressed by benchmarking the approximate results against consistent SARA calculations for a locally reacting finite length body of revolution. [Work supported by ONR.]

\section{8:15}

3aSA2. Scattering of a narrow acoustic beam by a truncated fluid-filled cylindrical shell. Stirling S. Dodd, Charles M. Loeffler (Adv. Sonar Group, Appl. Res. Lab., Univ. of Texas at Austin, P.O. Box 8029, Austin, TX 78713-8029), and Thomas A. Griffy (Univ. of Texas at Austin, Austin, TX 78713)

Previously a method of describing spherical acoustic waves in cylindrical coordinates was applied to the problem of point source scattering by an elastic infinite fluid-filled cylindrical shell [S. Dodd and C. Loeffler, J. Acoust. Soc. Am. 97, 3284 (A) (1995)]. This method is applied to numeri- cally model monostatic oblique incidence scattering from a truncated cylinder by a narrow-beam high-frequency imaging sonar. The narrow-beam solution results from integrating the point source solution over the spatial extent of a line source and line receiver in the frequency domain. The cylinder truncation is treated by the method of images, and assumes that the reflection coefficient at the truncation is unity. The scattering form functions, calculated using this method, are applied as filters to a narrow bandwidth, high $k a$ pulse to find the time domain scattering response. The time domain pulses are further processed and displayed in the form of a sonar image. These images compare favorably to experimentally obtained images [G. Kaduchak and C. Loeffler, J. Acoust. Soc. Am. 97, 3289 (A) (1995)]. The impact of the $s_{0}$ and $a_{0}$ Lamb waves are vividly apparent in the images.

\section{8:30}

3aSA3. The effect of ribs on the scattering response of a ribbed finite elastic cylinder. Angie Sarkissian (Naval Res. Lab., Washington, DC 20375)

The scattered field is examined from a finite cylindrical shell with ribs for $2.5<k a<25$, where $k$ is the wave number and $a$ is the radius of the cylinder. The cylinder contains ribs of two different sizes. Results for the monostatic, forward, and bistatic geometries will be shown where the interaction of the elastic waves with ribs produce extrema in the frequency/ angle domain. The rib spacing contains two types of periodicity. The first is representative of the spacing between any two ribs while the second is representative of the spacing between any two long ribs. This gives rise to two types of Bragg diffraction effects produced by the interaction of the acoustic field with the ribs. 
3aSA4. Acoustic scattering from a hemicapped cylindrical shell with substructures. Weiping Zhang (Component Products Group, Motorola, 1301 E. Algonquin Rd., Schaumburg, IL 60196), Takeru Igusa, and Jan D. Achenbach (Northwestern Univ., Evanston, IL 60208)

This paper extends the study on acoustic radiation of axisymmetric submerged shells of finite length to the scattering problem. A modal-based method is developed to determine the scattering from finite shells insounified by plane incident waves. A surface variational principle provides the acoustic impedance relationship between the surface pressure and displacement fields, which are expanded into a series of shape functions. Lagrange multipliers are introduced in a Lagrange formulation of the dynamic analysis to account for the interaction between the shell and internal substructures. Surface and far-field pressures scattered from the elastic shell are calculated. It is found that, at certain angles, the scattered pressure field has pronounced peaks, particularly as the incident frequency increases. A wave number domain analysis is performed on the surface displacement field. A dominant wave number is found which accounts for the high directivity pattern of the far field. The effect of substructures on the far field is also investigated.

\section{9:00}

3aSA5. On the scattering of sound from a submerged thin walled cylinder coated with a compliant layer. Jacek Jarzynski, John R. Bogle, George S. McCall (George W. Woodruff School of Mech. Eng., Georgia Inst. of Technol., Atlanta, GA 30332-0405), Michael D. Gray, and Gary W. Caille (Georgia Tech Res. Inst., Atlanta, GA 30332-0810)

There is currently an abundance of both mathematical and finiteelement models of the scattering of underwater sound from cylindrical bodies treated with compliant coatings. However, there is a significant lack of corresponding scattering measurements. This paper presents the results of a set of simple measurements of the monostatic backscatter from a coated, thin, ribbed cylindrical shell with flat endcaps for $k a$ of 2-8. The coating layer had an input impedance approximately one-fifth that of water, providing a near pressure-release boundary condition. Of particular interest is a minimum in the measured target strength which results from the resonant interaction of the coating and the shell. Results from finiteelement and modal expansion models of the coated shell are also presented with the aim of providing a physical understanding of the resonance. [Work supported by ONR.]

\section{9:15}

3aSA6. High-frequency threshold processes for leaky waves on cylinders of variable thickness: Fluid shell case. S. F. Morse, Z. W. Feng, and P. L. Marston (Dept. of Phys., Washington State Univ., Pullman, WA 99164-2814)

To develop an understanding of high-frequency scattering processes by complicated elastic objects, it is helpful to identify benchmark problems which exhibit new kinds of leaky wave scattering contributions. It is found that scattering by a nonconcentric circular cylinder exhibits scattering processes not present in the simple case of uniform thickness. By application of a cylindrical harmonic addition theorem, the scattering was computed for a right circular fluid cylinder with a circular off-set vacuum core. The speed of sound in the fluid corresponded to the longitudinal velocity for steel and exceeded the speed for the outer fluid (water). For a uniformthickness cylinder of sphere, previous calculations show a backscattering signature as the frequency was raised above the propagation threshold for the leaky waveguide mode analogous to the $s_{1}$ Lamb wave [P. L. Marston, Phys. Acoust. 21, 1-234 (1992), Sec. 4.11]. When the thin part of a variable thickness shell is on the shadow side, the vicinity of the $s_{1}$ mode threshold exhibits a new backscattering process due to mode cut off in the thin region and reversal of the associated ray. The local decrease in backscattering due to the thickness resonance is also affected. [Work supported by ONR.]
3aSA7. A unique boundary integral approach for acoustic radiation of axisymmetric bodies with arbitrary boundary conditions. W. Wang, N. Atalla, and J. Nicolas (G.A.U.S., Dept. of Mech. Eng., Univ. of Sherbrooke, Sherbrooke, PQ J1K 2R1, Canada)

A boundary integral method for solving the exterior acoustic radiation problem of axisymmetric bodies with arbitrary boundary conditions has been developed. The new formulation derives from the method proposed by Burton and Miller, which uses a linear combination of the Helmholtz integral equation and its normal derivative to ensure the uniqueness of the numerical solution at all frequencies. By taking advantage of the properties of axisymmetric geometry, and using the expansion of the boundary conditions and the surface distribution functions in Fourier series with respect to the angle of revolution, the surface integral is reduced to a line integral along the generator of the body, and Fourier integrals of the Green's function and its derivatives over the circumferential angle. A main feature of this formulation is that new recurrence formulas of the Fourier coefficients have been developed to evaluate accurately the Fourier integrals with the singular kemels in terms of the complete elliptic integrals. In order to demonstrate the validity and accuracy of the method, numerical results with quadratic isoparametric curvilinear elements are presented for radiation problems of a pulsating sphere, an oscillating sphere, and a finite cylinder with various boundary conditions.

\section{9:45}

3aSA8. Radiation of sound by a point-excited free-flooded cylindrical shell of finite length. K. Steven Kim (Signatures Directorate, Carderock Div., Naval Surface Warfare Ctr., Bethesda, MD 20084-5000)

Radiated acoustic pressure is obtained when a free-flooded cylindrical shell of finite length is excited by a time-harmonic point force. Integral equations are formulated for an elastic thin shell with simply supported boundary condition at both ends. Near-field and far-field acoustic pressures are calculated and cases of a short shell and a long shell are discussed.

\section{0:00-10:15 Break}

\section{0:15}

3aSA9. Interior noise of an aircraft fuselage: Dynamics and structural acoustics with experimentation. Peter C. Herdic, ${ }^{\text {a) }}$ Brian H. Houston, and Earl C. Williams (Naval Res. Lab., Code 7136, Washington, DC 20375-5350)

The area of interior noise is of growing interest to the aircraft industry due to recent trends associated with increasing passenger comfort. In an effort to understand the physics of the problem, highly spatially sampled surface motion and interior acoustic measurements of a business jet fuselage section under point excitation were made over a band from 10 to 1000 $\mathrm{Hz}$. Both the cylindrical shell and endcaps were measured using laser Doppler vibrometry and the associated coupling of these structural modes to interior pressure levels is observed and discussed. Superimposed on this interior response are the normal cavity modes of the interior which set up due to the quasirigid boundaries. Localization of the fulselage vibrations with the associated passbands and stop bands are found due to the pseudoperiodic nature of the internal structure of the fulselage. These structural acoustic attributes may be exploited to reduce the interior acoustic levels. a) Also at Sachs/Freeman Associates, Inc., 1401 McCormick Dr., Landover, MD 20785. 
3aSA10. Direct global stiffness matrix method for thin cylindrical shell dynamics. Mark A. Hayner and J. Robert Fricke (MIT, Rm. 5-435, 77 Massachusetts Ave., Cambridge, MA 02139)

This paper introduces the direct global stiffness matrix (DGSM) method for analyzing the dynamics of cylindrical shells with axial symmetry. The dynamic stiffness matrix of a straight, circular cross-section cylindrical element is formulated in terms of ring forces and displacements at each end on a per circumferential mode basis. The stiffness matrix is based on an exact wave-type solution of the thin shell Donnell equations. More complicated shell systems are analyzed by assembling any number of cylindrical elements. For example, a cylinder with a gradual variation in radius may be modeled by a series of shell elements welded together at their ends. Total system dynamics are found at any given frequency by inverting the global system matrix formed from the individual stiffness element matrices and summing over circumferential mode number. Results for a test case are presented to demonstrate and verify the implementation. [Research sponsored by ONR.]

\section{$10: 45$}

3aSA11. Power flow between circular cylindrical shells and flat plates. Benjamin F. Willis and Courtney B. Burroughs (Graduate Prog. in Acoust., Penn State Univ., State College, PA 16804)

An analytic model for the transmission of power between circular cylindrical shells and flat plates attached along a circumference of the shell is developed. The model includes transverse displacements associated with bending waves and in-plane displacements associated with longitudinal and shear waves. Plates internal and external to both semi-infinite and infinite shells are modeled. Examples of power transmission coefficients for different incident wave types in the plate, circumferential mode numbers and frequencies are presented and discussed.

\section{1:00}

3aSA12. Wave propagation on coated cylindrical shells. J. Gregory McDaniel (Bolt Beranek and Newman Inc., 70 Fawcett St., Cambridge, MA 02138)

This presentation describes methods of using an approximate elasticity formulation to compute natural wave numbers of waves in coated cylindrical shells. The efficient computation of these wave numbers is critical to coating design. A previously developed displacement-based variational formulation for coated shells [J. G. McDaniel and J. H. Ginsberg, J. Appl. Mech. 60, 463-469 (1993)] retains the accuracy of analytical formula- tions, but avoids the computational burdens associated with special functions of complex argument. This formulation, which was previously applied to two-dimensional problems, is extended to address wave propagation in the axial coordinate for each circumferential harmonic. Because the formulation is energy based, one has ready access to the strain energy distributions of each wave. For a specified real axial wave number, one obtains an easily solvable generalized eigenvalue problem for complex natural frequency. For a specified real frequency, the search for complex axial wave number is iterative. A fast algorithm for finding the complex axial wave numbers, which facilitates the design process, will also be discussed.

\section{1:15}

3aSA13. Active control of vibration transmission in a cylindrical shell. Xia Pan and Colin H. Hansen (Dept. of Mech. Eng., Univ. of Adelaide, Adelaide, South Australia 5005)

Active control of vibratory power transmission in a semi-infinite cylinder, using a circumferential array of control forces and a circumferential array of error sensors, is investigated experimentally and theoretically. The model considered is a semi-infinite cylinder, free at one end, anechoically terminated at the other end, and excited by an array of in-phase primary forces arranged in a line around its circumference. Control is achieved by an array of independent control forces applied downstream from the primary forces. For three or more control forces it is possible to achieve levels of power transmission reduction in excess of $30 \mathrm{~dB}$ for both acceleration and power transmission cost functions, provided that the error sensors are in the far field of the primary and control forces. The study also demonstrates how it is possible to simplify power transmission measurement methods for a realistic control force configuration.

\section{$11: 30$}

3aSA14. Acoustics of fluid-filled pipes in fluid-filled bores. Rama Rao V. N. (Dept. of Ocean Eng., MIT, Cambridge, MA 02139)

The acoustic transmission characteristics of fluid-filled elastic pipes inside fluid-filled bores in an infinite elastic solid are examined here. This model is of interest in exploratory drilling to study the propagation of drilling-related noises within the borehole and into the surrounding rock. The axially symmetric solutions in the four media (fluid inside the pipe, pipe, fluid in the annulus and the surrounding solid) are coupled through the boundary conditions. The resulting characteristic determinant is $9 \times 9$. The dispersion behaviors of the various modes are analyzed. The limiting behaviors at low and high frequencies are investigated. The cases of the surrounding solid being acoustically "fast" or "slow" compared to the fluid in the annulus are also examined. 
Session 3aSC

\title{
Speech Communication: Anatomy and Acoustics in Speech
}

\author{
Stephen A. Zahorian, Chair \\ Electrical and Computer Engineering, Old Dominion University, 543 Virginia Avenue, Norfolk, Virginia 23508
}

Contributed Papers

9:00

3aSC1. Vocal tract shapes and area functions from magnetic resonance imaging (MRI). Brad $\mathrm{H}$. Story, Ingo R. Titze, and Eric A. Hoffman (Univ. of Iowa, Iowa City, IA 52242)

There have been considerable research efforts in acoustic modeling of speech but there is still only a small body of information regarding direct three-dimensional measurements of the vocal tract shape. The purpose of this study was to acquire, using MRI, an inventory of three-dimensional vocal tract airway shapes that correspond to a particular set of vowels and consonants. The MR imaging was carried out for one subject (29-yr-old male, native of the midwestern United States) using a GE Signa 1.5-T scanner. The images were reconstructed and analyzed with a general display and quantitation package. The resulting three-dimensional (3D) vocal tract shapes were analyzed to find the cross-sectional areas perpendicular to the centerline extending from the glottis to the mouth to produce an "area function." These area functions were then used as input to a wavereflection type model of one-dimensional acoustic-wave propagation in the vocal tract. 3D reconstructions and area functions of the vocal tract shapes will be shown and the corresponding simulations of the speech sounds will be demonstrated.

\section{9:15}

3aSC2. A three-dimensional solution of the wave equation in a model of the vocal tract. Fariborz Alipour and Brad H. Story (Dept. of Speech Path. and Audiol. \& Natl. Ctr. for Voice and Speech, Univ. of Iowa, Iowa City, IA 52242)

The wave equation was solved in a cylindrical coordinate system for models of the vocal tract corresponding to vowels, /a/, /i/, and $/ \mathrm{u} / \mathrm{A}$ straight cylindrical model of vocal tract was built upon area function data for each vowel. Using boundary fitted coordinates, the vocal tract shape and its boundary conditions were simplified to a straight tube. However, this simplification in geometry resulted in a complicated wave equation in the new coordinate system. The transformed wave equation was discretized in space over a $91 \times 21$ grid and solved in time using a finite difference method. Preliminary results indicate that pressure contours are typically planar, however, higher-order mode propagation is possible with this model. The results of the model have been compared and validated against the open-open and closed-open uniform tubes with good accuracy. The predicted frequency response of this model was also compared with a wave-reflection type of model. [Work supported by NIDCD Grant No. DC00831-04.]

\section{9:30}

3aSC3. Driving a self-oscillating vocal fold model with laryngeal muscle activations. Ingo R. Titze (Natl. Ctr. for Voice and Speech, Dept. of Speech Path. and Audiol., Univ. of Iowa, Iowa City, IA 52242)

A self-oscillating model of the vocal folds (with arbitrary numbers of masses in the length, thickness, and depth dimensions) is driven by inputs that closely resemble muscle activations. With this model, EMG recordings and lung pressures can be used to simulate speech. In the present version, adduction-abduction and vocal fold tensing are probed to demonstrate the dynamics of laryngeal posturing and the corresponding phonation regimes.

9:45

3aSC4. Bifurcations in excised larynx experiments. David A. Berry, Ingo R. Titze, Brad H. Story (Dept. of Speech Path. and Audiol., Natl. Ctr. for Voice and Speech, Univ. of Iowa, Iowa City, IA 52242), and Hanspeter Herzel (Technical Univ. Berlin, Hardenbergstr. 36, D-10623 Berlin, Germany)

Bifurcation analysis is applied to vocal fold vibration in excised larynx experiments. In particular, two-dimensional bifurcation diagrams are generated in a parameter plane spanned by subglottal pressure and asymmetry of vocal fold adduction or elongation. Various phonatory regimes are observed, including single vocal fold oscillations. Selected spectra demonstrate a correspondence between the observed phonatory regimes and vocal registers noted in the literature. Many instabilities or bifurcations are noted in the regions of coexistence, i.e., the regions where the phonatory regimes overlap. Bifurcations are illustrated with spectrograms and fundamental frequency contours. Where possible, results from these studies are related to clinical observations. [This research was supported by Grant No. P60 DC00976 from the National Institute on Deafness and Other Communication Disorders.]

\section{0:00}

3aSC5. Determination of chaos in human vowels. Casey $R$. Winke and P. G. Vaidya (Dept. of Mech. and Mater. Eng., Washington State Univ., Pullman, WA 99163)

Human speech is a topic of much study today and many aspects of human speech are still unknown. The existence of chaos in a sustained vowel is one example. An analysis of the time series data generated by human speakers will be performed to determine if the data are chaotic. A set of pseudostate vectors will be created and used to analyze the time series data. From this analysis the largest Lyapunov exponent and the fractal dimension will be estimated. Also, support of a nonlinear origin of speech will be provided.

\section{0:15}

3aSC6. Articulatory kinematics from the standpoint of automatic speech recognition. Igor Zlokarnik (Los Alamos Natl. Lab., CIC-3, MS B256, Los Alamos, NM 87545)

The discriminant power of articulatory movements was evaluated for six subjects on a speaker-dependent continuous speech recognition task using a hidden-Markov-model-based speech recognition system. The articulatory measurements were gathered by means of electromagnetic articulography and describe the movement of small coils fixed to the speakers' tongue, jaw, and lower lip during the production of 108 German sentences. Four different articulatory representations were evaluated: coil displacements and their first three time derivatives (coil velocities, accelerations, and jerks). From these four representations, the coil accelerations performed by far the best in terms of recognition performance, both with 
and without acoustic features. The superior performance of acceleration features is surprising from the viewpoint of automatic speech recognition based on acoustics, since in the acoustic domain, acceleration features perform worse than static features on speaker-dependent tasks. From the viewpoint of articulatory phonetics, however, this result confirms the importance of the role articulator forces play in speech production.

\section{0:30}

3aSC7. Variability in tongue kinematics in stop production. Anders Lofqvist and Vincent L. Gracco (Haskins Labs., 270 Crown St., New Haven, CT 06511-6695)

The focus of this study is individual differences in tongue kinematics in the production of sequences of vowel-stop consonant-vowel. Four subjects produced VCV sequences with all possible combinations of the vowels $/ i, a, u$, and the stop consonants /p, t, k, b, d, g/. A magnetometer system was used to track vertical and horizontal movements of receivers placed on four points on the tongue. Individual variations were found for the influences of stop consonant voicing and vowel quality on tongue kinematics. In addition, in sequences with an alveolar consonant $/ \mathrm{t}, \mathrm{d}$ occurring between two high vowels $/ i, w$, some speakers lowered the rear portions of the tongue for the consonant closure, while other subjects maintained a tongue configuration that closely resembled that for the high flanking vowels. Possible accounts for these differences, such as oral anatomy, speaking rate, and speaking style will be discussed, as well as the acoustical consequences of the different articulatory patterns. [Work supported by NIH.]

\section{0:45}

3aSC8. Local and glohal effects of the pyriform fossa on speech spectra. Jianwu Dang (ATR Human Information Res. Labs., 2-2 Hikaridai Seikacho, Soraku-gun, Kyoto 619-02, Japan) and Kiyoshi Honda (ATR Human Information Res. Labs., Kyoto, Japan and Univ. of Wisconsin, Madison, WI 53705-2280)

The pyriform fossa consists of two bilateral cavities located behind the laryngeal ventricle. The fossa is known to act as a branch of the vocal tract by Fant's study (1960) based on $x$-ray data. This study investigates acoustic characteristics of the fossa using hardware models, which replicated the portion from the vocal folds to the velum based on volumetric MRI data, and using human subjects. Acoustic measurement was performed on the models by filling and emptying the fossa. The results confirmed that the fossa contributes local zeros to speech spectra at about $4 \mathrm{kHz}$. Further, it was shown that the effects of the fossa extend down to the first and second vowel formants. This global effect of the fossa on formant pattern has been confirmed by this simulation as being dependent on the location of the fossa close to the sound source. An in vivo experiment was performed by injecting water into the fossa of human subjects, and identified the antiresonance frequencies of the fossa as the frequencies increase with decreasing the volume. The zeros obtained from these experiments were found to be consistent with those in natural speech. The relatively stable acoustic properties are thought to provide one of the individual characteristics of speech sounds.

\section{1:00}

3aSC9. Open-loop acoustic-to-articulatory mapping. F Zussa, Q. Lin, G. Richard, D. Sinder, and J. Flanagan (CAIP Ctr., Rutgers Univ., Piscataway, NJ 08855-1390)

A new design is presented for open-loop estimation of articulatory parameters from an acoustic speech signal. Previous systems for acousticto-articulatory mapping generally involve an optimization loop which adapts synthetic speech to an arbitrary speech input. The open-loop method (i.e., without any optimization), aims to provide an accurate estimation of articulatory parameters at a low computational cost. The technique is used as a component of an adaptive voice mimic system. Model shapes corresponding to the natural signal are found by searching a precomputed table. The table associates vocal tract shapes to their corresponding spectra obtained by linear acoustic simulation. A new metric based on poles from linear predictive analysis is proposed to compare the natural spectrum to the precomputed synthetic spectra. Nearly real-time processing is achieved on a workstation by introducing a two-step search strategy. The resulting representation is known to provide an efficient parametrization of the speech signal which can be used for speech synthesis, low-bit-rate coding, and speech recognition. Further, the open-loop method also provides an accurate initial guess for traditional closed-loop adaptation. Initial results of speech coding using this open-loop estimation are presented and discussed. [Research supported by ARPA-DAST 63-93-C-0064.]

\title{
Session 3aUW
}

\section{Underwater Acoustics: Matched Field Processing}

\author{
Zoi-Heleni Michalopoulou, Chair \\ Mathematics Department, New Jersey Institute of Technology, University Heights, Newark, New Jersey 07102-1982
}

\section{Contributed Papers}

8:00

3aUW1. Experimental evaluation of matched-field track-beforedetect processing in shallow water. Paul A. Baxley (Ocean and Atmospheric Sciences Div., Acoust. Branch, NCCOSC RDTE Div. Code 541, 53560 Hull St., San Diego, CA 92152-5001)

The detection of a moving source at low signal-to-noise ratio can be enhanced by taking advantage of the time evolution of matched-field ambiguity surfaces. By including a track search in the matched-field process, track-before-detect (TBD) techniques extract source track information so that the target signal function can be reconstructed, rendering it more detectable. The detection enhancement produced by a shift-then-average (coherently and incoherently) TBD algorithm [Baxley, J. Acoust. Soc. Am. 90, 1844(A) (1993)] is investigated for multitone source-tow data recorded near San Diego during the first and third shallow-water evaluation cell experiment (SWellEX-1 and 3). The 3-knot source-tow traversed a rangeindependent radial from a FuP-mounted verical line array. Previously masked targets were rendered detectabie in ambient noise and in the presence of a single loud interferer. Gains in detectability of up to $5 \mathrm{~dB}$ above that obtained by straight averaging (no tracking) were obtained for a 6-min averaging time. 
3aUW2. A minimum variance unbiased estimator for resolving blurred beamformed images corrupted by signal-dependent speckle noise. Nicholas C. Makris (Naval Res. Lab., Washington, DC 20375)

A variety of methods currently exist for resolving the ambiguity and blurring introduced by beamforming ocean acoustic line array measurements of ambient noise [R. A Wagstaff, J. Acoust. Soc. Am. 63, 863-869 (1978)] and reverberation [N. C. Makris, J. Acoust. Soc. Am. 94, 983-993 (1993)]. However, these methods are not necessarily optimal because they assume that the measured data are deterministic, whereas in actuality they are stochastic. An optimal estimator for resolving such blurred beamformed images produces the minimum variance possible and is unbiased in its output. Estimation theory is used to derive such a minimum variance unbiased (MVU) estimator and to determine bounds on the resolution of such blurred images. The fields measured by the array are assumed to obey circular complex Gaussian random (CCGR) statistics, which have previously been shown to describe a wide variety of ocean acoustic field measurements from towed-array reverberation, to both horizontal and vertical ambient noise. Given CCGR fields, the resulting beamformed images are corrupted with signal-dependent noise known as speckle. Coherence theory for CCGR fields is used to express the MVU estimator for resolving blurred images in terms of the temporal coherence of the received fields and the measurement time.

\section{8:30}

3aLWW3. Detection performance of broadband adaptive matched-field beamforming on a vertical array in shallow water. Newell O. Booth and Philip W. Schey (Ocean and Atmospheric Sciences Div., NCCOSC RDTE DIV Code 541, San Diego, CA 92152-5001)

Quantitative estimates of detection performance are presented for a broadband adaptively processed vertical line array with matched-field replicas. The estimates were obtained with broadband and multitone signal data obtained in the shallow water evaluation cell experiment 3 (SWellEx3). The experiment was carried out in July 1994 west of Point Loma in 200 m deep water of complex bathymetry. The multitone test signal was transmitted from an acoustic source towed at various depths over complex propagation paths and consisted of five sets of ten tones, each set spanned $50-200 \mathrm{~Hz}$. Each set was transmitted at various levels over $22-\mathrm{dB}$ dynamic range. The beamforming used sets of 8 and 16 hydrophones over $80-\mathrm{m}$ vertical aperture. Detection performance is estimated for various input signal-to-noise ratios by processing sets of frequencies corresponding to the various signal levels. Another set of 10 frequencies which excludes the signal frequencies form the noise only estimates. [Work supported by ONR, Code 321US.]

\section{8:45}

3aUW4. HLA/VLA broadband adaptive beamforming detection performance comparison in shallow water. William S. Hodgkiss, James J. Murray, Gerald L. D'Spain, Katherine H. Kim (Marine Physical Lab., Scripps Inst. of Oceanogr., La Jolla, CA 92093-0701), Newell O. Booth, Philip W. Schey, and Joe Rice (NCCOSC RDTE DIV Code 541, San Diego, CA 92152-5001)

A broadband adaptive beamforming detection performance comparison is presented for a vertical array with matched-field replica vectors and a horizontal line array with plane-wave replica vectors. The data analyzed consists of both multitone $(50-200 \mathrm{~Hz})$ and full broadband $(75-150 \mathrm{~Hz})$ transmissions recorded during SWellEx-3 (shallow water evaluation cell experiment 3). SWellEx-3 was carried out in July 1994 west of Point Loma in approximately 200 -m-deep water. A MPL vertical array was deployed from the R/P FLIP and the NRaD SWSS (shallow water sensor string) horizontal line array was co-deployed $500 \mathrm{~m}$ to the east. Source tows (multitone transmissions) and stations (full broadband transmissions) were carried out at various levels spanning a 22 - and 16-dB dynamic range, respectively. Radar data will be included indicating the location and dynamics of interfering noise sources from shipping traffic in the area. [Work supported by ONR, Code 321 US.]
3aUW5. Selective high-resolution environmental parameter estimation using sector-focused matched-field processing. Howard A. Chandler, Christopher Feuillade, and George B. Smith (Naval Res. Lab., Code 7173, Stennis Space Center, MS 39529-5004)

Sector-focusing (SF) has been shown to be effective at enhancing range-depth resolution while minimizing the instability caused by environmental mismatch. It has also been shown that a stable Bartlett type response could be obtained with SF using a small sector in range-depth space. This property is used to advantage when matched-field processing is used for source localization. By extending the sector focusing concept to include environmental parameter variation within the sector, high resolution may be selectively achieved. In this way greater resolution may be realized for a chosen set of search parameters by including in the sector, replicas derived from a set of "neighboring" environmental parameters. Conversely, by not including replicas derived from other "known". parameters, such as source range or depth, a broad Bartlett-like response may be obtained. Thus the estimate will simultaneously attain high resolution only in the selected search parameter while remaining insensitive to measurement errors in all other parameters, including errors in source location. Examples are presented for a simple parametrization scheme using the KRAKEN normal mode model to simulate a canonical shallow-water environment.

\section{9:15}

3aUW6. Improvement of signal-to-noise ratio at the single-sensor for narrow-band signals in a partially correlated noise field. D. $R$. Munn and Stergios Stergiopoulos (Dept. Elec. Eng., Technical Univ. of Nova Scotia, P.O. Box 1000, Halifax, NS B3J 2X4, Canada)

This paper deals with the development of a processing technique that will improve the signal-to-noise ratio at the single sensor for a received signal that is embodied in a partially correlated noise field. The approach of this study is unique in that the noise will be treated as being nonwhite and partially correlated. The concept of the proposed development is based on the time interval over which the temporal coherence or correlation properties of a noise field are defined. For narrow-band signals, the associated temporal coherence period is much longer than the correlation time interval of the anisotropic noise field. Thus a coherent integration of discontinuous segments of received signals will enhance the signal-to-noise ratio at the sensor by lowering the correlation properties of the associated nonwhite noise. Reconstruction by the proposed technique of the narrowband signal time series with improved signal-to-noise ratio at the sensor will allow the use of the existing high resolution techniques to be utilized more effectively by lowering their threshold values in order to detect very weak signals. The intention here is to integrate the characteristics of the real anisotropic noise field during the preliminary processing stages of the array-sensor received signals.

\section{9:30}

3aUW7. Jovian acoustic matched-field processing. Michael D. Collins, B. Edward McDonald (Naval Res. Lab., Washington, DC 20375), W. A. Kuperman (Scripps Inst. of Oceanogr., La Jolla, CA 92093), and William L. Siegmann (Rensselaer Polytechnic Inst., Troy, NY 12180)

Hubble Space Telescope images contain evidence of outward propagating waves. Infrared images from the fragment $G$ impact site contain a small circular feature (the inner ring) and a large circular feature (the outer ring). The inner ring (which appears in several images) has been identified as an internal gravity wave based on its observed group speed of about 450 $\mathrm{m} / \mathrm{s}$ [Hammel et al., Science 267, 1288-1296 (1995)]. The radius of the outer ring (which appears in only a few images) is consistent with the group speeds of acoustic modes trapped in the Jovian sound channel. During a 34-min interval between images, however, the outer ring appears to expand at only $425 \mathrm{~m} / \mathrm{s}$, which is about half the minimum group speed of acoustic waves. To properly analyze the expansion of the outer ring, it is necessary to account for the vertical component of propagation. This is 
not necessary for the inner ring, which is apparently dominated by a single gravity wave mode trapped in the water clouds [Ingersoll and Kanamori, Nature 374, 706-708 (1995)]. Since acoustic waves repeatedly enter and exit the debris cloud as they cycle about the sound channel axis, one would expect the outer ring to spread outward (and vaporize the debris cloud as it goes) at a variable rate. Matched-field processing results are in agreement with the observations for an acoustic source located deep in the water clouds.

\section{$9: 45$}

3aUW8. Source localization in the Gulf of Mexico. Zoi-Heleni Michalopoulou, Michael B. Porter (Dept. of Mathematics and Ctr. for Appl. Marhematics and Statistics, New Jersey Inst. of Technol., Newark, NJ 07102), and Jack lanniello (Naval Undersea Warfare Ctr., New London, CT 06320)

Data collected during the Gulf of Mexico experiment were processed for the estimation of the source location and the bottom depth of the water column at the site of the experiment. The data consist of time series received at five vertically separated hydrophones after the transmission of a pulse in the 100- to $600-\mathrm{Hz}$ frequency band. Successful estimation of the parameters of interest was achieved with simulated annealing. Using the calculated parameter estimates in simulations, a remarkable match was observed between real and synthetic data.

\section{0:00-10:15 Break}

\section{$10: 15$}

3aUW9. The dependence of ocean acoustic parameter resolution bounds on sample size. Nicholas C. Makris (Naval Res. Lab., Washington, DC 20375)

It is currently common practice in theoretical ocean acoustics to derive "fundamental" parameter resolution bounds for a monochromatic measurement of the temporally fluctuating field received on a hydrophone array. However, a monochromatic measurement corresponds to a single random sample. In applied ocean acoustics, single samples are seldom if ever used for parameter estimation because the associated error can be unnecessarily large. Instead estimates are derived from ensemble averages such as the sample covariance. To bridge the gap between these two approaches, the Fisher information for the sample covariance is examined and found to be equal to the number of independent and stationary samples times the Fisher information for a single sample. Therefore, there are no practical limits on parameter resolution if (1) the bound for a single sample is finite, which is generally the case of interest, (2) a sufficiently large population of independent samples can be found. The parameter resolution issue then becomes one of determining the maximum number of such samples. This number is set by physical variables that do not appear in the monochromatic or instantaneous measurement. A means of determining this number from the temporal coherence of the received field and the measurement time is given.

\section{$10: 30$}

3aUW10. Coherent wideband matched-field processing in an uncertain environment. Stephen P. Czenszak and Jeffrey L. Krolik (Dept. of Elec. Eng., Duke Univ., Box 90291, Durham, NC 27708)

Broadband matched-field processing approaches have centered around incoherent averaging of narrow-band MFP ambiguity surfaces. This technique works well when environmental mismatch results in a frequencyindependent shift in the source position. This appears to be true for mismatch in the bottom parameters when the sound speed is constant in the lower half of the water column. In the presence of more general soundspeed profiles, the performance of incoherent averaging techniques is significantly degraded. In this paper, coherent wideband matched-field processing is proposed to exploit statistical dependencies between the signal wavefronts at different frequencies for more general settings. The fourthorder statistics of the data are used to exploit these cross-frequency dependencies. For Gaussian data this fourth-order moment can be expressed in terms of the narrow-band second-order data cross-spectral density matrices. The coherent wideband MV-EPC processor consists of minimizing the squared output power of the beamformer subject to a set of constraints designed to provide robustness over an ensemble of random environmental realizations. In the presence of sound-speed mismatch this processor is shown to yield improved probability of correct localization performance versus incoherently averaged wideband matched-field processors. [Work supported by ONR.]

\section{$10: 45$}

3aUW11. Experimental demonstration of environmental source tracking. Laurie T. Fialkowski, John S. Perkins, Michael D. Collins, W. A. Kuperman (Naval Res. Lab., Washington, DC 20375), and John A. Fawcett (SACLANT Undersea Res. Ctr., 19038 La Spezia, Italy)

Environmental source tracking (EST) is a matched-field processing (MFP) technique that exploits source motion in a complex environment [Collins et al., J. Acoust. Soc. Am. 94, 3335-3341 (1993)]. EST involves searching for a source track which provides the best agreement with the data. EST performed using a single receiver and a single frequency can often outperform conventional MFP methods with a fixed source and an array of receivers. In this paper EST performance is discussed, using a data set obtained in shallow water north of Darwin, Australia. In-situ acoustic data from a known source were used to invert for the environmental parameters. Parabolic equation solutions were then used to generate replica fields throughout the region. EST was performed for both radial and nonradial source tracks. In some instances, the source speed was reasonably constrained to eliminate very short tracks from the search space. The performance of several error measures is discussed. Improved EST results were obtained using multiple receivers and frequencies.

\section{1:00}

3aUW12. Saturated ocean acoustic intensity statistics as a function of temporal coherence and measurement time. Nicholas C. Makris (Naval Res. Lab., Washington, DC 20375)

Coherence theory is used to analyze the statistics of saturated ocean acoustic intensity measurements. The circular complex Gaussian random field assumption has been used to describe saturated multipath propagation in the ocean for many years. However, previous analysis of intensity statistics in the saturated region have implicity been limited to certain special cases for which the time-bandwidth product of the field measured from a given source is unity. In this paper, the intensity statistics in the saturated region are extended and generalized to be a function of measurement time and temporal coherence. As a result, the well-known 5.6-dB transmission loss (TL) standard deviation of Dyer is found to be a special case of a more general TL standard deviation that approximates $4.34 \sqrt{1 / \mu} \mathrm{dB}$ when the time-bandwidth product or number of independent samples $\mu$ of the intensity average is large. Therefore, the TL standard deviation is shown to approach zero when the time-bandwidth product becomes large, as it must in this deterministic limit. A similar generalization is obtained for the TL mean. Additionally, asymptotic analysis shows that the log-normal distribution for intensity can provide an excellent approximation to TL statistics contrary to previous contentions.

\section{$11: 15$}

3aUW13. Broadband model-based processing for shallow ocean environments. James V. Candy (Lawrence Livermore Natl. Lab., Univ. of Califomia, P.O. Box 808, L-495, Livermore, CA 94550) and Edmund J. Sullivan (Naval Undersea Warfare Ctr., Newport, RI 02841)

Most acoustic sources found in the ocean environment are spatially complex and broadband. When propagating in a shallow ocean these source characteristics complicate the analysis of received acoustic data considerably. On the other hand. each of the narrow-band lines composing the broadband source spectrum can be considered multiple observations which can be used to enhance signal levels. The usual approach is to process each line separately and combine the results to achieve more enhancement at the array than that which could be utilized for a single 
temporal frequency. The enhancement of broadband acoustic pressure-field measurements using a vertical array is discussed. Here the model-based approach is developed for a broadband source using a normal mode propagation model. It is well known from propagation theroy that a different modal structure evolves for each temporal frequency line; thus it is not surprising that the model-based solution to this problem results in a scheme that requires a "bank" of model-based processors-each processing its own underlying modal structure for the narrow frequency band it operates over. It is shown how this broadband processor can be implemented in pseudo-real-time due to its underlying parallel structure.

\section{1:30}

3aUW14. A DSP-based neural computing system for acoustical signal processing. J. W. Zhang and Y. L. Ma (P.O. Box 19, Northwestern Polytechnical Univ., Xían, 710072, China)

An artificial neural network (ANN) can be implemented by many methods, such as electronic hardware or simulation on digital machines. Hardware implementation of an ANN cannot provide network size and computation flexibility because its topology is difficult to change once it is implemented in the hardware. Simulation of an ANN on commercial parallel computers is not cost effective because their architectures are not optimized for ANN computation, while software approaches need a large amount of execution time. The solution to these problems is to use the special parallel-processing architectures. Based on studying existing methods, this paper concentrates on the DSP-based virtual implementation of ANN. A parallel processing system composed of TMS320C 30 has been designed and configured, which meets the needs of ANN application to acoustical signal processing in the real world. It is a multiprocessor system with shared multiport memories. In this paper, the architecture of the system is described, and its performance is evaluated. The scalibility and communication method are also studied. The simulation results show that parallel efficiency of the system has reached a high level $80 \%$ when running a BP algorithm for classification of acoustical signals.

\section{1:45}

3aUW15. Prediction of mismatch dependence and source depth dependence of mismatch-induced peak broadening in matched-field processing. George B. Smith (Naval Res. Lab., Stennis Space Center, MS 39529-5004) and Nichalos D. Gardner (University of Mississippi, University, MS 38677)

In previous work [Smith and Akundi, J. Acoust. Soc. Am. 95, 2980(A) (1994); Smith, Akundi, and Gardner, J. Acoust. Soc. Am. 97, 3292(A) (1995)] analytic expressions for mismatch-induced range and depth localization error and signal gain degradation for a linear correlator matchedfield processor were obtained. Those results agreed with previously obtained results [Shang and Wang, J. Acoust. Soc. Am. 89, 2285-2290 (1991)]. In this work the problems of mismatch-induced peak broadening and the mismatch dependence and the source depth dependence of this peak broadening are addressed. Computer simulations are compared to predictions based on the hypothesis that the peak broadening in range and in depth are related to the variances of the distributions of the range shifts and depth shifts, respectively, predicted by the mode pairs. [This work was supported by the Office of Naval Research and by the Mississippi Alliance for Minority Participation (MAMP) program.]

\title{
Meeting of Accredited Standards Committee S2 on Mechanical Vibration and Shock
}

to be held jointly with the

\section{U.S. Technical Advisory Group (TAG) Meeting for ISO/TC 108 Mechanical Vibration and Shock}

\author{
D. J. Evans, Chair S2 \\ National Institute of Standards and Technology (NIST), Acoustics, Mass and Vibrations Group, Building 233, Room A147,
} Gaithersburg, Maryland 20899

D. F. Muster, Chair, U.S. Technical Advisory Group (TAG) for ISO/TC 108, Mechanical Vibration and Shock 4615 O'Meara Drive, Houston, Texas 77035

\begin{abstract}
Standards Committee S2 on Mechanical Vibration and Shock. Working group chairs will present reports of their recent progress on writing and processing various shock and vibration standards. There will be a report on the interface of S2 activities with those of ISO/TC 108 (the Technical Advisory Group for ISO/TC 108 consists of members of S2, S3, and other persons not necessarily members of those committees), including a report on the activities of ISO/TC 108, with the plans for its September 1996 meeting in Sydney, Australia. A report will be given on the meetings of ISO/TC 108/SC3 and ISO/TC 108/SC5, held at BSI in London, U.K., during September 1995.
\end{abstract}

Scope of S2: Standards, specifications, methods of measurement and test, and terminology in the fields of mechanical vibration and shock, and condition monitoring and diagnostics of machines, but excluding those aspects which pertain to biological safety, tolerance and comfort. 


\title{
Session 3pID
}

\section{Interdisciplinary: Hot Topics in Acoustics}

\author{
Joseph Pope, Chair \\ Pope Engineering Company, P.O. Box 236, Newton Center, Massachusetts 02159
}

A special session on "Hot Topics in Acoustics" is presented at each meeting of the Society. From several of the Society's technical committees, technical specialty and interdisciplinary groups, a member is chosen to present a tutorial paper on topics of current special interest. The presentations are intended to help acousticians become familiar with issues and achievements that are not within their own primary field of interest.

Chair's Introduction-1:20

Invited Papers

1:25

3pID1. Hot topics in physiological acoustics. Donald W. Nielsen (Central Inst. for the Deaf, $818 \mathrm{~S}$. Euclid Ave., St. Louis, MO 63110)

For those in the Society whose main interest lies in other acoustic fields, the presentation will review current topics in physiology of audition. Much basic physiological research has been focused on the micromechanics of the cochlea, the end organ of hearing. For many years the cochlear tuning process was through to be passive and mechanical; however, recent studies indicate that active motion of one group of cochlear hair cells adds to and sharpens the tuning. The results and implications of studies of outer hair cell motion will be discussed. Also discussed will be the cochlear implant, a device that addresses injury or loss of cochlear hair cells, the cause of most incurable forms of deafness. With this device, the electric fields of electrodes surgically implanted in the cochlea stimulate the nerve supply in the absence of normal hair cells. Also presented will be recent advances in molecular biology that attempt to induce growth or regrowth of hair cells in cochleae that have none.

1:45

3pID2. Hot topics in physical acoustics. Anthony A. Atchley (Phys. Dept., Naval Postgrad. School, Monterey, CA 93943)

The field of physical acoustics involves: (1) the use of our understanding of the parameters governing propagation of acoustic waves in and interaction of acoustic waves with other systems to understand the structure and behavior of those systems; (2) the study of nonlinear acoustic propagation and phenomena; and (3) the use of acoustics to generate or stimulate physical processes. This hot topics presentation will draw on examples from each of these categories to demonstrate both the breadth of the field and how physical acoustics is closely tied to many other technical areas within the Society. Some specific topics are sonoluminescence, thermoacoustics, high intensity ultrasonic surgery, sonic booms, imaging at the nanometer scale, resonant ultrasound spectroscopy, and applications of modulated radiation pressure to fluid mechanics.

2:05

3pID3. Hot topics in animal bioacoustics. Whitlow W. L. Au (Hawaii Inst. of Marine Biology, Univ. of Hawaii, P.O. Box 1106, Kailua, HI 96734)

The field of animal bioacoustics is a diverse one involving a wide variety of species varying from insects and birds to terrestrial and aquatic animals. Some of the interesting research being performed with different species will be highlighted. Recent research has uncovered a possible new mechanism for sound localization in the parasoidal fly. Acoustics is being used to solve agricultural problems associated with insects. The U.S. Air Force is conducting a comprehensive program to study the effects of loud sounds and noise on different birds and terrestrial mammals. The availability of the U.S. Navy Integrated Undersea Surveillance System for detecting, localizing, and tracking whales has contributed significantly in the understanding of the seasonal distribution and movements of these large mammals. The ATOC program has generated considerable controversy on the possible effects of the ATOC source on marine animals. Several research programs have been initiated to investigate this particular issue. 


\title{
Session 3pSC
}

\section{Speech Communication: Studies of Voice}

\author{
Keith A. Johnson, Chair \\ Department of Linguistics, Ohio State University, 222 Oxley Hall, 1712 Neil Avenue, Columbus, Ohio 43210-1298
}

Contributed Papers

1:00

3pSC1. Analysis and perception of voice similarities among family members. Rachel E. Kushner (MGH-Inst, of Health Professions, 101 Merrimac St., Boston, MA 02114) and Corine A. Bickley (MIT, Cambridge, MA 02139)

The similarities of voice between members of the same family were found to be high when measured both perceptually and acoustically. The recordings of nine subjects (two mothers, two daughters, two sisters, two brothers, and an unrelated male) were made using sentences of different stress and reiterant syllable combinations. The recordings were paired into related and unrelated sets. Unfamilar listeners were instructed to listen to the recordings and rate their similarity on a numerical scale. Results showed that paired voices of those who were related showed a significant number of high scores as opposed to the voiced pairs of individuals who were unrelated. It was also found that voice similarity was more easily detected when the listeners heard whole sentences as opposed to reiterant syllables and individual words. Other influential factors associated with high scores (similar sounding voices) were equal prosody and volume. Related pairs who had similar prosody scored higher than unrelated pairs with similar prosody. Acoustic measurements of individual voices found that the difference in amplitude of the first and second harmonic as well as the spectral tilt were related to the listener's judgment about voice similarities.

\section{1:15}

3pSC2. Voice quality: Listener identification of African-American versus Caucasian speakers. Sonja A. Trent (Dept. of Psych., Univ. of South Florida, 4202 E. Fowler Ave., Tampa, FL 33620)

Although relative contributions of dialect and voice quality are difficult to tease apart, previous research has shown that listeners can perceive the gender and ethnicity of speakers at better than chance performance from recorded speech samples. The present study attempted to mitigate dialect cues and measure the resulting effects on perception by screening for dialect and by varying stimulus length. In experiment $I$, passages produced by ten African-American males, ten African-American females, ten Caucasian males, and ten Caucasian females were presented to 40 listeners with the same gender/ethnic distribution. In experiment II, sentence and citation $/ \mathrm{hVd} / \mathrm{stimuli}$ produced by five of the speakers from each of the gender/ethnic groups were presented to 40 listeners. Both experiments required listeners to categorize the speakers by gender and ethnicity. Listeners were able to identify speaker gender and ethnicity at far better than chance performance. Accuracy of identification increased with stimulus length. Accurate identifications were made even in the citation condition, suggesting a unique contribution of voice quality apart from dialect. Caucasian male speakers were most accurately identified, while AfricanAmerican females were least accurately identified. No listener gender/ ethnic group performed more accurately than another. [Work supported by NIDCD.]
$1: 30$

3pSC3. Voice analysis: A stochastic approach. Yingyong Qi (Dept. of Speech and Hearing Sciences, Univ. of Arizona, Tucson, AZ 85721)

Current methods of computing noise present in human voices depend upon being able to determine fundamental periods. In this paper, a method of estimating noise present in human voices that does not depend on the determination of the boundaries between fundamental periods is described. In this method, noise was computed as the uncorrelated component in the voice signal. The magnitude of the uncorrelated component was obtained by computing the average, maximum cross correlation between many pairs of short frames of voice signals. The method is evaluated using synthetic and natural voice signals. Results indicate that it is an effective tool for estimating the noise component in voice signals. [Work supported by NIH.]

\section{$1: 45$}

3pSC4. Acoustic study of acted emotions in speech. Rong Wang and William J. Strong (Dept. of Phys. and Astronomy, Brigham Young Univ., Provo, UT 84602)

An extensive set of carefully recorded utterances provided a speech database for investigating acoustic correlates among eight emotional states. Four actors and four actresses simulated the emotional states of anger, joy, neutral, nervousness, hate, fear, sadness, and depression. Many acoustic parameters were extracted from analyses of the $64(8 \times 8)$ emotion portrayals. A reduced set of acoustic parameters was obtained by eliminating some highly correlated parameters. The talkers realized "unique" emotions, such as anger, with consistent values of the parameters. Different talkers realized "ambiguous" emotions, such as neutral and nervousness, with different values of the parameters. Emotion "pairs," such as sadness and depression, had similar parameter values. The acoustic parameters tended to correlate strongly with "unique" emotions but less well with "ambiguous" emotions.

\section{2:00}

3pSC5. Vocal expression of emotion is associated with formant characteristics. Jo-Anne Bachorowski (Dept. of Psych., Vanderbilt Univ., Nashville, TN 37240) and Michael J. Owren (Reed College, Portland, OR 97202)

Acoustic properties of speech likely provide external cues about internal emotions, a phenomenon called "vocal expression of emotion." Most empirical work in this area has emphasized global measures, such as pitch, speech rate, and overall amplitude. In this work, associations between induced positive and negative emotions and more fine-grained formant characteristics were tested. Subjects were 120 undergraduates who completed a questionnaire measuring their typical intensity of emotional experience and thereafter performed a challenging lexical-decision task during which noncontingent feedback occurred. On each of 10 baseline and 20 on-task trials, subjects spoke the words "Test $n$ test" (n referred to next trial or trial block). The intent was to induce positive and negative emotions by providing success and failure feedback, and to measure the ex- 
pression of emotion during the experience of those states. Analysis focused on the 3 phoneme from the first "test." Frequency and amplitude of $F 1$, $F 2$, and $F 3$ were examined with MANOVAs that examined changes between baseline and on-task trials, as well as differences associated with the feedback conditions. Subjects who reported experiencing emotions intensely were found to show statistically significant changes in formant characteristics between baseline and on-task trials in both feedback conditions.

WEDNESDAY AFTERNOON, 29 NOVEMBER 1995

ST. LOUIS E, 1:00 TO 2:50 P.M.

\title{
Session 3pUW
}

\section{Underwater Acoustics and Acoustical Oceanography: Bubble Effects}

\author{
Frank S. Henyey, Chair \\ Applied Physics Laboratory, HN-10, University of Washington, 1013 N.E. 40th Street, Seattle, Washington $98105-6698$
}

Chair's Introduction-1:00

\section{Contributed Papers}

$1: 05$

3pUW1. High-frequency propagation in a bubbly medium. Ralph R. Goodman (Appl. Res. Lab., Penn State Univ., State College, PA 16804), Jerald W. Caruthers, Stephen J. Stanic, and Ming Y. Su (Naval Res. Lab., Stennis Space Center, MS 39529)

During a shallow-water, direct-path experiment off the Panama City, Florida coast a storm passed through the area that gave rise to both a change in sound speed of about $50 \mathrm{~m} / \mathrm{s}$ and pulse-to-pulse variations that were significantly larger than those found during relatively benign periods before and after the storm. The depth of the water was about $10 \mathrm{~m}$ with the propagation path over a range of about $60 \mathrm{~m}$ at mid-depth. Very narrow beam pulsed signals from 20 to $135 \mathrm{kHz}$ were transmitted at 1 -s intervals. Measurements of the void fraction of bubbles showed a bubble concentration that can account for the change in the speed of sound. Fluctuations in the arrival times can be attributed to small changes in the average bubble concentration along the propagation path. Both scattering and dispersion due to the presence of small bubbles will be discussed. Comparison of measurements before, during, and after the storm period will be presented. [Work supported by NRL and ONR.]

1:20

3pUW2. The collective acoustic properties of water containing resonating air bubbles, and its effect on the propagation of sound. C. Feuillade (Naval Res. Lab., Stennis Space Center, MS 39529-5004)

The classic theory of sound propagation in bubbly water incorrectly describes the properties of dense media containing resonating bubbles of uniform size. It assumes that the bubbles oscillate independently. However, resonating bubbles are strongly coupled by acoustic radiation, and acoustic propagation can be fully explained only in terms of the collective action of the medium, which is dominated by the "symmetric" normal mode. In this work, the propagational characteristics of bubbly water are determined by averaging the ensemble behavior of the symmetric mode over distributions of bubble sizes and locations. All orders of multiple scattering are included, and "shadowing" effects incorporated. New theoretical expressions for the phase speed and attenuation are presented. Comparisons between theory and experimental data are made by integrating multiple scattering effects over a "region of collective interaction" around the bubbles. For uniformly size bubbles, a downward frequency shift and suppression of the attenuation peak is observed, which is more pronounced for higher volume fractions. The phase speed is also modified. In water containing many differently sized bubbles, multiple scattering typically plays a much smaller role in determining acoustic properties, and the classic theory of propagation is adequate.

\section{$1: 35$}

3pUW3. Sound propagation through a bubbly wake. Holly Burch, Michael Buckingham, and Svein Vagle (Marine Physical Lab.-0238, Scripps Inst. of Oceanogr., 9500 Gilman Dr., La Jolla, CA 92093-0238)

The presence of bubbles in the near surface layer of the ocean causes significant reduction of the sound speed (A. B. Wood, 1957). The result is an upward-refracting sound-speed profile which acts as an acoustic waveguide. An experiment was performed in Saanich Inlet off the south east coast of Vancouver Island to investigate the spectral structure of sound which had propagated through a bubble layer. A motorboat, driven in a circle, created a bubbly wake which advected through the experimental region. Acoustic measurements were obtained at depths of 1.5 and $3.5 \mathrm{~m}$ at a range of $10 \mathrm{~m}$ from a broadband source. The spectral structure of the data depends on depth, but in both cases, shows a regular positioning of the peaks. The Green's function solution for an isovelocity sound-speed profile (Lloyd's mirror) and an upward-refractng inverse-square profile were fitted to acoustic spectra. The bubble-free records are well fitted by the Lloyd's mirror theory, and good inverse-square fits were found for acoustic "snapshots" of the bubbly field at 2 and $8 \mathrm{~min}$ after field generation. It is possible to invert for the sound-speed profile in the near-surface bubble layer by fitting the spectral structure predicted by the inverse-square theory to that of sound that has propagated through the bubbly field.

\section{$1: 50$}

3pUW4. Rough sea surface scattering in the presence of an upward-refracting bubble layer. Kenneth $E$. Gilbert, Kathy $K$. Bekker, and Lucy J. Kulbago (Appl. Res. Lab. and the Graduate Prog. in Acoust., Penn State Univ., P.O. Box 30, State College, PA 16804)

Perturbation theory for scattering from rough surfaces is generalized to account for nonplanar insonifying waves. The generalized theory is applied to low-frequency $(50-1500 \mathrm{~Hz})$ sea surface scattering in the presence of an upward-refracting bubble layer. Using an exact numerical solution for the insonifying wave, it is shown that although the sea surface backscatter is enhanced by the upward-refracting bubble layer, the enhancement is not nearly enough to account for the experimentally observed backscatter. Several analytic approximations (plane wave, WKB, Snell's law) are considered to gain some physical insight into the numerical calculation. At frequencies below a few hundred Hertz, the plane-wave approximation is adequate, that is, the bubble layer becomes acoustically transparent. At higher frequencies, the WKB approximation, which conserves energy, is surprisingly accurate. At low grazing angles and all frequencies, simply using the local wave number (i.e., Snell's law) is a very poor approximation that does not conserve energy and grossly overestimates the effects of upward refraction. It is concluded that even with upward refraction 
present, scattering from the rough air-water interface is a minor contributor to the total acoustic backscatter. [Work supported by the Office of Naval Research.]

3pUW5. A discussion of the low-frequency resonance scattering of a bubble cloud. Paul A. Hwang (Res. Div., QUEST Integrated, Inc., 21414 68th Ave. S., Kent, WA 98032)

A comparison of four different formulas to compute the lowest mode resonance frequency of a bubble cloud is provided in this presentation. Based on the comparison, it is concluded that only one of the four equations represents the generalized solution, which includes air bubbles as its asymptotic condition, of void traction equals unity. Also shown is the numerical calculation of the scattering of a spherical bubble cloud using the classical solution of acoustic scattering of elastic spheres. With the elastic properties of the bubble cloud approximated by Wood's formula. tion, it is found that isothermal conditions exist only at a very low void fraction level (less than 0.0001 ). Within the range of void fraction from 0.001 to 0.1 , the polytropic coefficient of the bubble cloud is approximately 1.2, which is halfway between adiabatic and isothermal conditions. Finally, two simple scaling laws for the resonance characteristics of a spherical bubble cloud are presented: (1) the dimensionless resonance wave number is uniquely determined by the void fraction; and (2) the backscatter cross section at resonance is uniquely determined by the resonance frequency. [Work supported by ARPA.]

\section{$2: 20$}

3pUW6. Monopole acoustic radiation by a bubble encountering a turbulent flow field. Ali R. Kolaini and Alexei Goumilevski (Natl. Ctr. for Physical Acoust., Univ. of Mississippi, University, MS 38677)

The acoustical characteristics of an "adult" bubble encountering a turbulent flow are studied both exprimentally and theoretically. By injecting an "adult" bubble in a flow, generated by a submerged axisymmetric water jet, the acoustic reexcitation of the bubble with and without breakup may occur in the shear-induced flow region. Bubbles of various sizes were introduced into jets of various speeds by means of interchangeable hypodermic needles. Results of the role of the turbulent flow characteristics in determining the acoustic bubble response are discussed. The characterizations of both acoustical and the dynamics of bubbles encountering the turbulent flow field depend upon the estimated integral and microlength scales, the corresponding Reynolds numbers, and the cirtical Weber numbers for both bubble distortion and breakup. These parameters are examined both in fresh and salt water. A simple model is given to account for bubble acoustic radiation. The Rayleigh-Plesset equation was altered by incorporating the turbulent pressure fluctuation of the flow as a driving force of the bubble. Some conclusions are given about the nature of the turbulent flow field and conditions under which the bubble acoustic reexcitation may occur. [Work supported by ONR.]

$$
\text { 2:35 }
$$

3pUW7. Scattering of sound from laboratory breaking waves. Yi Mao, Ali R. Kolaini, Xinwei Hao, and Pat B. Dandenault (Natl. Ctr. for Phys. Acoust., Univ. of Mississippi, University, MS 38677)

Computer-controlled gravity waves were generated to break in an anechoic tank. A sequence of 15 incident bursts was directed at the bubble cloud entrained by each breaking wave. A burst has seven cycles of a sine wave of frequency ranging from 10 to $15 \mathrm{kHz}$. The interval between adjacent bursts is set at $0.1 \mathrm{~s}$ in order to avoid overlapping the bursts. By analyzing the data of the 15 sound scattering events, the fluctuation was significantly reduced. The bubble clouds were observed to be roughly semicylindrical. The bubble size distribution in an entrained cloud was obtained from video images of a bubble cloud. This experimental information of the shape, size, and bubble size distribution of a bubble cloud was employed as the input into the theory adapted from Sarkar and Prosperetti [J. Acoust. Soc. Am. 93, 3128-3138 (1993)] to estimate the theoretical scattering strength. A comparison between the experimentally measured and the theoretically estimated scattering strengths will be shown. [Work supported by ONR.]

\title{
Plenary Session, Business Meeting, and Awards Ceremony
}

\author{
Robert E. Apfel, Chair \\ President, Acoustical Society of America
}

\author{
Business Meeting \\ Presentation of Certificates to New Fellows and Science Writing Award Recipients
}

Presentation of Awards

Pioneers of Underwater Acoustics Medal to William A. Kuperman

Silver Medal in Engineering Acoustics to James E. West

von Békésy Medal to Peter Dallos

Wallace Clement Sabine Medal to A. Harold Marshall

Presentation of the Lifetime Achievement Award of the American Auditory Society to Edgar Villchur 


\title{
Session 4aAB
}

\section{Animal Bioacoustics: Startle Responses}

\author{
Charles R. Greene, Jr., Cochair \\ Greeneridge Sciences, Inc., 4512 Via Huerto, Santa Barbara, California 93110 \\ Mardi C. Hastings, Cochair \\ Department of Mechanical Engineering, Ohio State University, 206 West 18th Avenue, Columbus, Ohio 43210-1107
}

Chair's Introduction-8:00

Invited Papers

8:05

4aAB1. The physiology and psychophysics of the acoustic startle reaction. James R. Ison (Dept. of Brain and Cognitive Sci., Meliora Hall, Univ. of Rochester, Rochester, NY 14627)

Intense noise bursts elicit in many animals, including humans, an abrupt and graded contraction of the flexor muscles called the "acoustic startle reflex" (ASR). Response vigor is determined in part by the acoustic properties of the eliciting stimulus, and in part by organismic factors such as biological rhythıns, habituation, and emotion. Additionally it is powerfully affected by diverse and apparently irrelevant momentary shifts in the stimulus surround occurring just prior to the eliciting stimuli. These stimuli, called "prepulses," can variously inhibit ("PPI") or facilitate ("PPF") the ASR, the general effect being "reflex modification." The major variables affecting the strength of RM are prepulse salience and lead time, and, for short lead times, whether prepulses are increments or decrements in background level. Reflex modification has obvious intrinsic interest, and is being used to study sensory, perceptual, and cognitive processes in laboratory animals and in humans. The short brain-stem pathway responsible for ASR elicitation is simple and reasonably understood, with few remaining unknowns. In contrast, reflex modification consists of a set of less well understood semi-independent phenomena, which may variously call on different levels of the neuraxis, from brain stem to cortex, in processing stimulus input. [Work supported by NIH.]

8:35

4aAB2. Attentional factors in the elicitation and inhibition of the startle reflex. Howard S. Hoffman (Dept. of Psych., Dalton Hall, Bryn Mawr College, Bryn Mawr, PA 19010)

More than a century ago, Helmholtz noted the "curious fact" that by mere conscious effort one can focus attention on any portion of the visual field and that the process "is entirely independent of the position and accomodation of the eyes." A sequence of experiments in the Bryn Mawr laboratory, provided strong support for Helmholtz's assertion. When eyeblinks were elicited by a tap between the eyebrows, the response was inhibited by a light flash (i.e., a prepulse) presented in various parts of the visual field. Subjects were able to modify the amount of inhibition engendered by the flash by shifting their attention toward or away from its impending location and they were able to do so without moving their eyes. A second experiment examined the effects engendered by foreknowledge as to the modality (auditory versus tactile) of an impending startle eliciting stimulus. Foreknowledge was found to reduce response amplitude. In other experiments foreknowledge as to when a startle eliciting signal would occur also reduced response amplitude. Overall the results imply that foreknowledge acts selectively with respect to the modality of a given sensory input and it also acts selectively as to where in the nervous system that input is directed.

4aAB3. Startle reflex in fish. Peter H. Rogers, Thomas N. Lewis (George W. Woodruff School of Mech. Eng., Georgia Inst. of Technol., Atlanta, GA 30332-0405), and Michael D. Gray (Georgia Tech Res. Inst., Atlanta, GA 30332-0810)

Directional hearing in fish is a poorly understood phenomenon, whose complexity makes it difficult to analyze. The directional, Mauthner cell mediated, startle response, which does not involve the CNS, is considerably simpler and more amenable to analysis but may still provide insight into the algorithms and mechanisms for more general directional hearing tasks. The startle reflex is modeled and studied experimentally in goldfish. The basic model posits that the initial polarity of both the incident acoustic pressure and particle acceleration measured by the fish's auditory system determines the direction of a threat, and initiates an escape reflex in the appropriate direction. The startle reflex of goldfish is observed experimentally in a large acoustic test tank at Georgia Tech. The subject is placed in the center of the tank, and its behavior is observed using a video camera. The acoustic stimulus is generated using simple spherical sources driven to provide independent control of pressure and velocity waveform, so that, for example, monopole and nearfield dipole fields could be established. A parallel effort was undertaken in order to determine a potentially relevant acoustical stimulus, the field generated by an attacking fish. [Work supported by ONR.] 
4aAB4. The neural mechanism for directional escape in the goldfish. Robert C. Eaton, Audrey L. Guzik, and Janet L. Casagrand (Ctr. for Neurosci., EPO Box 334, Univ. of Colorado, Boulder, CO 80309)

In response to sudden sound, many fishes rapidly accelerate away from the stimulus. This complex behavior, or C-start, is mediated by a network of brain-stem neurons that receive acoustic input and connect to motoneurons in the spinal cord. In the brain-stem network, the bilateral pair of Mauthner cells (M-cells) play the major role in determining the initial direction of the C-start. Each M-cell axon crosses the brain and connects to motoneurons on the opposite side of the body, so that the animal turns away from the side of the activated M-cell. M-cells receive primary acoustic afferents and inhibitory input from a network of "PHP" cells. PHP cells have a very short latency response to sound and operate in a feedforward mode to regulate $M$-cell firing threshold. These studies suggest that the PHP cells receive specific combinations of pressure- and displacement-sensitive auditory afferents that inhibit the M-cell to sounds coming from the side of the body opposite the stimulus. Thus only the correct M-cell fires, while its opposite counterpart is inhibited by PHP cells. Present studies involve an electrophysiological, behavioral, and neurocomputational analysis of this hypothesis. [Work supported by NIH and ONR.]

\section{0:05-10:15 Break}

\section{Contributed Papers}

\section{0:15}

4aAB5. Effects of helicopter noise on spotted owls: Methodology. Larry L. Pater (U.S. Army Construction Eng. Res. Labs., Champaign, IL), David K. Delaney, Teryl G. Grubb (USDA Forest Service, Flagstaff, AZ), Paul Beier (Northern Arizona Univ., Flagstaff, AZ), and M. Hildegard Reiser (Holloman Air Force Base, NM)

The Endangered Species Act requires federal agencies to assess impacts of their activities on threatened and endangered species and to carry out programs for the conservation of listed species. In some cases activities, including military training, are curtailed because of potential impacts. There is currently no known published research on the possible impact of noise on the spotted owl. The present research addresses the question of the noise impacts of low level helicopter flights and ground activities such as chain saws and motorcycles on Mexican spotted owls (Strix occidentalis lucida). This research will characterize the effect of anthropogenic activity on breeding Mexican spotted owls by developing a dose-response threshold model that quantifies animal response relative to sound stimulus levels and approach distances. Consideration is given to the hearing range and sensitivity of the Mexican spotted owl, the sound level received at roost and nest sites, flight response of nonbreeding owls, effects on nest attentiveness of breeding females and on the rate of prey delivery by breeding males, and development of disturbance-specific management guidelines to minimize potential audio and visual impacts from helicopter and ground activities.

\section{0:30}

4aAB6. Responses of bowhead whales to sonobuoy impacts. Charles R. Greene, Jr. (Greeneridge Sciences, Inc., Santa Barbara, CA 93110) and William Koski (LGL Ltd., Envir. Res. Assoc., King City, ON L7B 1A6, Canada)

Air-dropped sonobuoys have been used to record sounds near bowhead whales since 1979. They have not been observed to react to sonobuoys dropped $0.5-1 \mathrm{~km}$ from them during numerous studies conducted during the summer; however, on a few occasions they have reacted to sonobuoys air dropped near them during their spring and fall migrations. The sound from an AN/SSQ-57A sonobuoy impact was recorded at distance $800 \mathrm{~m}$ in water $90 \mathrm{~m}$ deep in the western Beaufort Sea. Hydrophone depths were 3 and $18 \mathrm{~m}$. Analysis of the impact signature and sound transmission paths revealed two reflected paths: one with a single bottom reflection and the second with two bottom reflections. A third arrival, occurring later and very weak, was also noted. The presence of a strong, low-frequency sound source nearby led us to high-pass filter the signal at $1 \mathrm{kHz}$. Analysis of the received signal amplitude, corrected for spreading loss, revealed a peak source pressure level of $211 \mathrm{~dB}$ re: $1 \mu \mathrm{Pa} / \mathrm{m}$. The positive acoustic impulse at a distance of $1 \mathrm{~m}$, assuming no positive pulse spreading and scaling the pressure for spherical spreading, is estimated to be $5.55 \mathrm{~Pa} / \mathrm{s}$. The overall sound duration of the single bottom bounce arrival was $0.9 \mathrm{~ms}$. [Work supported by MMS.]

\section{0:45}

4aAB7. The effects of the acoustic thermometry of ocean climate signals on dolphins and small whales. Whitlow W. L. Au, Paul E. Nachtigall, and Jeffrey L. Pawloski (Hawaii Inst. of Marine Biology, Univ. of Hawaii, P.O. Box 1106, Kailua, Hawaii 96734)

The acoustic thermometry of ocean climate (ATOC) program of Scripps Institute of Oceanography and the Applied Physics Laboratory, University of Washington, will broadcast a low-frequency $75-\mathrm{Hz}$ phasemodulated acoustic signal over ocean basins in order to study ocean temperatures on a global scale. One of the major concerns is the possible effect of the ATOC signal on marine life, especially on dolphins and whales. In order to address this issue, the hearing sensitivity of a false killer whale (Pseudorca crassidens) and a Risso's dolphin (Grampus griseus) to the ATOC sound was measured behaviorally. A staircase procedure with the signal levels being changed in 1-dB steps was used to measure the animals' threshold to the actual ATOC coded signal. The results will be used to examine how the ATOC sound will affect the hearing capability of these small cetaceans. The relative effect of the ATOC sound will also be compared with effects from other sources of noise such as aircraft, ocean going ships, pleasure craft, and other whales.

\section{1:00}

4aAB8. Acoustic stunning of marine animals. Mardi C. Hastings (Dept. of Mech. Eng., Ohio State Univ., Columbus, OH 43210-1107)

Acoustic stunning, a complete physiological shutdown of bodily functions, occurs in some marine animals when exposed to intense sound for a short period of time. Anecdotal evidence of acoustic stunning has been reported in the literature [G. E. MacGinitie and N. MacGinitie, Natural History of Marine Animals (McGraw-Hill, New York, 1968); K. S. Norris and B. Mohl, Am. Naturalist 122, 85-104 (1983)], but the physiological mechanisms and threshold values associated with this phenomenon have not been studied. Acoustic stunning of gouramis (Trichogaster trichopterus) was observed in the laboratory when they were exposed to intense pure tones underwater; however, oscars (Atronotus ocellatus) and goldfish (Carassius auratus) exposed to similar tones were not acoustically stunned. This paper examines the characteristics of the sound field that caused the transient stunning. In addition, differences in body size and 
geometry as well as in structure of the ear and lateral line among the three species are examined in an effort to explain why only the gourami was affected.

\section{1:15}

4aAB9. Ambient noise measurements of selected fish environments. Joseph A. Clark (CDNSWC, Code 734, Bethesda, MD 20084, and COMB, Ste. 236, Columbus Ctr., 701 E. Pratt St., Baltimore, MD 21202), Amrit N. Bart (COMB, Baltimore, MD 21202), and Jane A. Young (CDNSWC, Bethesda, MD 20084, and COMB, Baltimore, MD 21202)

A survey of ambient noise levels in a variety of environments, where fish are found or raised, has been conducted. Included in the survey were regions in the open water of the Chesapeake Bay, fish pens used by aqua farms in several states, and closed cycle tanks used for aqua-culture research and for public exhibitions. One-third octave-band and narrow-band measurements of the ambient noise levels will be presented. Sound recordings will also be played to demonstrate other characteristics of the noise at the different sites. [Work partially supported by Dual Use Technology Program, NAVSEA.]

\section{$11: 30$}

4aAB10. Active/passive technique for the creation of a low-frequency traveling wave inside a water-filled tube. James J. Finneran and Mardi C. Hastings (Dept. of Mech. Eng., Ohio State Univ., 206 W. 18th Ave., Columbus, OH 43210)

Studies of sound reception in aquatic animals are often complicated by the problem of generating a suitable acoustic stimulus at low frequencies underwater. In this study, active and passive control techniques were used to generate a traveling acoustic wave inside a water-filled acrylic tube, at pure-tone frequencies from 10 to $800 \mathrm{~Hz}$. The compliant walls of the tube reduced the wavelength and increased attenuation, both of which helped to reduce the amplitude of any reflected waves. Above $400 \mathrm{~Hz}$, a traveling wave was present without any active control. Below $400 \mathrm{~Hz}$, a secondary source was used to actively absorb sound at the tube end. The active control system consisted of two hydrophones, two sound projectors, and a personal computer with digital signal processing hardware. A simple pattern search algorithm was used to adjust the secondary source amplitude and phase in order to attain the desired acoustic impedance at the test location. This technique seems well suited for underwater sound tests on fish, since the relationship between pressure and particle velocity may be easily manipulated. [Work supported by ONR Grant No. N00014-94-10337.]

\title{
Session 4aPA
}

\section{Physical Acoustics and Bioresponse to Vibration and to Ultrasound: Lithotripsy}

\author{
E. Carr Everbach, Chair \\ Department of Engineering, Swarthmore College, 500 College Avenue, Swarthmore, Pennsylvania 19081-1397
}

Chair's Introduction-8:00

Invited Papers

8:10

4aPA1. Theoretical modeling of the acoustic pressure field produced by commercial lithotripters. Michalakis A. Averkiou, Lawrence A. Crum (Appl. Phys. Lab., Univ. of Washington, Seattle, WA 98105), and Mark F. Hamilton (Univ. of Texas, Austin, TX 78712-1063)

A theoretical model for the acoustic field produced by commercial lithotripters based on the KZK equation is presented. The KZK equation has been used previously to model high-intensity sound beams in thermoviscous fluids. Both electrohydraulic and piezoelectric lithotripters are considered. To model the acoustic field reflected from ellipsoidal reflectors found in electrohydraulic lithotripters, geometrical acoustics are used to define a directivity function at the mouth of the reflector. An equivalent focused source with a shading function defined by the directivity function is then assumed as the boundary condition for the KZK equation. A code that solves this equation entirely in the time domain is used to obtain results for the acoustic pressure. The numerical results are compared with previous experiments [Coleman et al., Ultrasound Med. Biol. 13(10), 651-657 (1987)] and good agreement is found. Results for propagation in both water and tissue are presented. Positive pressures of 40-100 MPa and negative pressures of 4-10 MPa are predicted in the focal region. A reflector with a pressure release surface is also considered. In this case the field close to the source is an inverted replica of that of the rigid reflector. However, strong distortion due to nonlinearity affects the rest of field. Negative pressures of 20-30 MPa are predicted in the focal region for this type of reflector. [Work supported by NIH.]

$$
\text { 8:35 }
$$

4aPA2. A review of current research on optical techniques for acoustic measurements in lithotripsy. Andrew J. Coleman (Medical Phys. Directorate, Guy's and St. Thomas' Hospital Trust, London SE1 7EH, UK) and Peter C. M. Galloway (Natl. Physical Lab., Teddington, Middlesex TWII OLW, UK)

This paper reviews some recent research on optical techniques for acoustic pressure measurement along with initial results obtained in the field of a newly developed electromagnetic-type shock wave source. A long-path-difference Michelson interferometer enables direct determination of the Doppler shift from a target to evaluate particle velocity in water in the range $1-100 \mathrm{~ms}^{-1}$. The instrument 
operates as a high-resolution spectroscope with a 532-nm wavelength laser source and a remote optical-head utilizing fiber links. The aim is to establish measurement standards in lithotripsy where conventional hydrophone-based procedures are inadequate. A second optical fiber ultrasound sensor, orginally developed for the measurement of photoacoustic transients in tissue, is also described [P. C. Beard and T. N. Mills, Proc. SPIE 2388, 446-457 (1995)]. This sensor employs a Fabry-Perot interferometer comprising a 50$\mu \mathrm{m}$ polymer film acoustically matched to water which is illuminated with light emerging from the fiber. An electromagnetic shock wave source developed recently by the Medical Physics Directorate (Guy's and St. Thomas' Hospital, London) has been used to compare measurements from the two interferometer systems with conventional hydrophone measurements.

4aPA3. Stress wave fracturing of biological stones with lithotripter pulses. Sheryl M. Gracewski, Girish Dahake, Adam Fitzgerald, and Stephen J. Bums (Mech. Eng., Univ. of Rochester, Rochester, NY 14627)

Stress waves contributions to stone fragmentation during lithotripsy are investigated both theoretically and experimentally. A two-dimensional finite difference scheme is developed to analyze the time evolution of the strain fields inside irregularly shaped solids subjected to ultrasonic pulses that simulate lithotripter shock waves. The reflections and superposition of stress waves inside the stones are analyzed to better understand the effects of stone parameters and geometry on the induced internal strains and fragmentation during lithotripsy. Numerical results show the focusing effect of the concave backsurface of a spherical stone, with the subsequent formation of focal zones (caustics). The focusing is reduced when a section of the back surface of the stone is removed. Principal strain contours depict the time evolution of the stress waves as they refract and reflect at the stone boundaries. Locations of maximum stresses are calculated and compared to locations of crack initiation in experiments with stones of similar geometry. The calculated time evolution of strain at fixed points within a stone is compared to imbedded silicon strain gauge measurements. Fracture characteristics of synthetic stones show internal crack initiation and subsequent propagation to external stone surfaces, indicative of intemal stress fragmentation mechanisms. [Work supported by NIH.]

\section{9:25}

4aPA4. The mechanical effects of focused shock waves on tissuelike structures. Bradford Sturtevant and Danny Howard (Graduate Aeronautical Labs., Calif. Inst. of Tech., Pasadena, CA 91125)

Shock waves focused to strengths sufficient to fracture kidney stones in extracorporeal shock wave lithotripsy (ESWL) injure soft tissue. In a complex organ like the kidney, the injury occurs in a sequence of processes beginning with the mechanical stimulus during or shortly after the passage of the shock wave, followed by a complex series of bio-mechano-chemical responses, terminating with altered function and histology of the organ. This paper presents preliminary results of an in vitro study of the initial mechanical stimulus. A planar nitrocellulose membrane of order $10 \mu \mathrm{m}$ thick immersed in liquid is used as a simple model of tissue membrane. Use of thoroughly degassed water, glycerin, and castor oil at the focus of an electrohydraulic lithotripter controls cavitation, and addition of $20-\mu \mathrm{m}$-diam hollow glass spheres to the test liquid simulates the acoustic nonuniformity of tissue. In water, small ( 1-mm diameter) circular punctures are generated by bubble collapse after only one or two shock waves. In noncavitating, uniform liquids the membrane only fails after of order 100 shock waves, by cavitation. With the addition of acoustic nonuniformity in order to increase shear induced by the shock, a different mode of failure occurs; the membrane fails in long $(\sim 1 \mathrm{~cm})$ tears after about 20 shocks. [Work supported by NIH Grant P01 DK43881-01A3.]

4aPA5. Analog experiments of tissue damage generated during ESWL treatments. K. Takayama, T. Kodama (Shock Wave Res. Ctr., Inst. Fluid Sci., Tohoku Univ., Sendai, Japan), M. Kuwahara (Miyagi Cancer Ctr.), and M. Ioritani (Tohoku Univ., Sendai, Japan)

Among the possible causes of ESWL tissue damage, the interaction of shock waves with cavitation bubbles is believed to be the most responsible. A series of analog experiments has been carried out for clarifying the mechanism of tissue damage during ESWL. Interactions of shock waves with single air bubbles in water were examined, including the formation of a rebound shock wave and the generation of liquid microjets. The collapse of air bubbles which were attached to gelatin walls and exposed to shock waves was quantitatively observed using double-exposure holographic interferometry and high-speed cinematography. In these analog experiments, shock waves were created via microexplosives: 10-mg silver azide pellets were pasted on the tip of an optical fiber and detonated with the radiation of a pulsed YAG laser beam. Details of the bubble collapse were well resolved. More complex materials analogous to human tissue have been tested and will be presented also.

$$
\text { 10:15-10:30 Break }
$$

4aPA6. Kidney tubular epithelial cell injury in shock wave lithoripsy: The search for an appropriate in vitro model. James A. McAteer, Andrew P. Evan (Dept. of Anatomy, Indiana Univ. School of Medicine, 635 Barnhill Dr., Indianapolis, IN 46202-5120), James E. Lingeman (Methodist Hospital of Indiana, Indianapolis, IN 46202), and Sharon P. Andreoli (Indiana Univ. School of Medicine, Indianapolis, IN 46202-5120)

Renal injury is a documented consequence of SWL and includes direct damage to the tubular epithelium. The factors of SW administration that contribute to cell injury are not fully understood, nor have the mechanisms responsible for cell damage been adequately characterized. Numerous laboratories have used cultured cells to assess SW cytolytic potential. For the most part, these studies have employed fully dissociated cells. To test the idea that cell polarity and cell-cell interactions influence response to SW, the proximal tubulelike line LLC-PK1 was prepared to allow isolation for SW exposure as a polarized monolayer (PM). SW response of PM was compared to dissociated cells. SW exposures were performed with an unmodified HM3 (electrohydraulic). Viability of 
dissociated cells descrased with increased SW number at $18 \mathrm{kV}$ but was equivalent when $\mathrm{kV}$ was varied (14-22 kV). ATP depletion was greater for freshly dissociated cells than for isolated cells from stable suspension culture. PM showed diminished lytic injury (LDH release) compared to dissociated cells. These findings indicate that the nature of an in vitro preparation influences the characteristics and severity of cell injury, and that polarized epithelial monolayers can be used to model the tubular cell response to shock waves. [Work supported by NIH P01 43881.]

\section{0:55}

4aPA7. Biological effects of lithotripter fields. Diane Dalecki, Sally Z. Child, Carol H. Raeman, and Edwin L. Carstensen (Dept. of Elec. Eng. and the Rochester Ctr. for Biomedical Ultrasound, Univ. of Rochester, Rochester, NY 14627)

Lithotripsy has become a common procedure for the treatment of kidney stones. Fields of lithotripters are capable of producing both stone distintegration and damage to soft tissues. Thresholds for biological effects of lithotripter fields include hemorrhage in mammalian lung ( $\sim 1.5 \mathrm{MPa})$, kidney (3-5 MPa), and intestine (1-3 MPa), malformations in the chick embryo $(<10 \mathrm{MPa})$, premature ventricular contractions in the frog heart (5-10 MPa), and killing of Drosophila larvae $(<1 \mathrm{MPa})$. Tissues containing gas bodies are particularly susceptible to damage. Pulsed ultrasound can also produce comparable soft-tissue damage and the similarity of threshold pressures for lithotripter and pulsed ultrasound exposures suggests that the same mechanisms may be involved in both phenomena. Cavitation and purely mechanical forces have been investigated as possible mechanisms for these bioeffects.

\section{Contributed Papers}

4aPA8. A durable, wide-bandwidth lithotripsy hydrophone. Byron Holz and E. Carr Everbach (Swarthmore College, Eng. Dept., 500 College Ave., Swarthmore, PA 19081)

In order to balance wide bandwidth with durability, a hydrophone for lithotripsy research was developed using disposable elements of 9$\mu$ m-thick PVDF copolymer film. Each element, which measures $1 \mathrm{~cm}$ $\times 10 \mathrm{~cm}$ and is stretched across a Plexiglas frame, contains $0.2-\mathrm{mm}$-wide electrodes overlapping in a crosshair pattern at the element center. When an element fails, it may be quickly replaced. To avoid the need for recalibration with each replacement, the use of hysteresis poling ensures constant sensitivity of elements [E. Carr Everbach, J. Acoust. Soc. Am. Suppl. $18 \mathbf{8 7}, \mathrm{S} 128$ (1990)]. Electronics located in the frame include a wide-bandwidth preamplifier and gating circuitry to prevent saturation by the electromagnetic pulse from a spark-gap lithotripter. This design provides the needed bandwidth to resolve shock wave frequency components beyond $100 \mathrm{MHz}$, as well as the durability and spatial resolution necessary to map the acoustic field within a lithotripter. [Work supported by an NSF PFF.]

\section{1:35}

4aPA9. Further study of the effects of shear viscosity on inertial cavitation thresholds. John Allen (Dept. of Mech. Eng., Univ. of Washington, Seattle, WA 98195), Ronald A. Roy (Univ. of Washington, Seattle, WA 98195), and Charles C. Church (San Diego, CA 92126)

Initial research [Allen et al., J. Acoust. Soc. Am. 96, 3306(A) (1994)] on accessing the effects of shear viscosity on inertial cavitation thresholds has been extended in order to further explicate previous results. Thresholds for inertial cavitation in water and biological media modeled as a viscous fluid were calculated using a numerical implementation of the Gilmore equation for adiabatic bubble oscillations [Church, J. Acoust. Soc. Am. 83, 2210-2217 (1988)]. The threshold criterion was chosen to be a bubble collapse temperature of $5000 \mathrm{~K}$ as to facilitate comparison with the analytical theory of Holland and Apfel [IEEE-UFFC 36, 204 (1989)]. The scaling of the calculated pressure thresholds with initial bubble radius was previously not sufficiently explained by linear resonance theory. The addition of calculations of the "nonlinear resonance" sizes, however, more adequately explains this scaling. Furthermore, the nonlinear resonance size is shown to be a more accurate indicator of the bubble sizes most likely to undergo inertial cavitation than the linear resonance size. The qualitative physics pertaining to these additional results is highlighted along with comparisons to recent experimental measurements [Zheng et al., J. Acoust. Soc. Am. 95, 2855(A) (1994)]. [Work supported by NIH through Grant No. ROI CA39374.]

\section{1:50}

4aPA10. Kidney size is a determinant of structural/functional injury following shock wave treatment of pigs. Andrew P. Evan, Bret A. Connors (Dept. of Anatomy, Indiana Univ. School of Medicine, 635 Barnhill Dr., Indianapolis, IN 46223), Lynn R. Willis (Indiana Univ., Indianapolis, IN 46223), Anne Trout, and James E. Lingeman (Methodist Hospital, Indianapolis, IN 46202)

It has been suggested that kidney size is a risk factor for increased shock wave lithotripsy- (SWL) induced damage. This relationship was investigated in pigs of two different ages; ten weeks of age (group 1) and five weeks of age (group 2). Each pig received 2000 shocks, $24 \mathrm{kV}$ to the right kidney. Glomerular filtration rate (GFR), PAH clearance and extraction were measured $1 \mathrm{~h}$ before and 1 and $4 \mathrm{~h}$ after SWL treatment. The kidneys were harvested at the end of the clearance study. Values for GFR, PAH clearance, and extraction were reduced in the shocked kidney of both groups $1 \mathrm{~h}$ post-SWL. Calculated values for true renal plasma flow showed a greater reduction in the treated kidneys of group 2 versus group 1 . The degree of subcapsular hemorrhage and intraparenchymal bleeding and tubular disruption was more extensive in group 2 versus group 1 treated kidneys. The data document the degree of structural and functional injury induced by SWL and support the hypothesis that the effect of SWL is greatest in kidneys having the least mass. [Work supported by NIH Grant No. P01 DK 43881.]

12:05

4aPA11. Deoxyribonucleic acid damage induced by a spark-gap lithotripter in vitro. Douglas L. Miller, Ronald $M$. Thomas, and Brian D. Thrall (M.S. P7-53, Battelle PNL, P.O. Box 999, Richland, WA 99352)

Chinese hamster ovary cells suspended at a concentration of $10^{6}$ $\mathrm{ml}^{-1}$ were exposed at $37 T^{\circ} \mathrm{C}$ in thin-walled chambers at the focus of a spark-gap lithotripter. Discharges of $20 \mathrm{kV}$, triggered at a $2-\mathrm{Hz}$ rate, emitted bright flashes of light, and generated shock waves of 27-MPa peak positive and 3.3-MPa peak negative pressures. DNA damage in viable cells was assessed using the single-cell gel electrophoresis (comet) assay. After exposure to 500 discharges, DNA strand breaks were readily detected. This DNA damage approximated the damage seen either from 10-min treatment with 5-micromolar hydrogen peroxide, or from $37 \mathrm{~J} / \mathrm{m}^{2}$ of $254-\mathrm{nm}$ ultraviolet light. Cavitation caused by the shock waves reduced cellular viability (trypan-blue exclusion) to about $67 \%$ after 500 discharges. However, the cavitation generated less than $0.1 \mu \mathrm{M} \mathrm{H}_{2} \mathrm{O}_{2}$, far too little to account for the DNA damage. Placing an opaque plastic sheet between the gap and the chamber to block the flashes of light eliminated the DNA damage effect, but maintained the cavitation-induced viability reduction (to about $66 \%$ after 500 discharges). This finding suggests that the ultraviolet light, 
not the cavitation, associated with the spark discharge may be the mechanism responsible for the DNA damage observed. [Work supported by NIH Grant No. CA42947.]

\section{$12: 20$}

4aPA12. Effects of frequency-dependent absorption on the propagation and attenuation of high-intensity acoustic waves containing shocks. Vera A. Khokhlova, Oleg A. Sapozhnikov (Dept. of Acoust., Phys. Faculty, Moscow State Univ., Moscow 119899, Russia), Michalakis A. Averkiou, and Lawrence A. Cnum (Univ. of Washington, Seattle, WA 98105)

Shock waves used in extracorporeal lithotripsy and ultrasonic surgery are greatly influenced by thermoviscous losses in the tissue. The influence of the frequency dependence of the absorption coefficient on nonlinear wave distortion and energy attenuation has not been investigated in detail up to now. For absorption frequency dependence other than quadratic, the corresponding nonlinear evolution equation is often complicated and thus frequency domain methods are more effective. The presence of the shock, however, makes this analysis time consuming due to the large number of spectral components needed in the numerical calculation. To avoid this complexity, a semianalytical method that enables calculations with relatively few harmonic components is used. Propagation in media with various power law absorption is considered. It is shown that energy attenuation of an initially sinusoidal wave in a medium with linear power law absorption is quite similar with that in a medium with quadratic power law absorption, even though the waveforms are quite different. The effect of selective absorption of a certain harmonic component is also investigated. It is shown that second harmonic selective absorption does not prevent shock formation, but results in much less energy attenuation over large distances. [Work supported by NIH, FIRCA, and RFFI.]

\title{
Session 4aPP
}

\section{Psychological and Physiological Acoustics: Auditory Physiology and Psychoacoustics Potpourri (Poster Session)}

\author{
Alec N. Salt, Chair \\ Department of Otolaryngalogy, Washington University School of Medicine, 517 South Euclid Avenue, \\ St. Louis, Missouri 63110
}

\section{Contributed Papers}

\begin{abstract}
All posters will be on display from 8:00 a.m. to 12:00 noon. To allow contributors an opportunity to see other posters, contributors of odd-numbered papers will be at their posters from 8:00 a.m. to 10:00 a.m. and contributors of even-numbered papers will be at their posters from 10:00 a.m. to 12:00 noon. To allow for extended viewing time, posters will remain on display until 8:00 a.m. on Friday morning.
\end{abstract}

4aPP1. Relaxation dynamics of spontaneous otoacoustic emissions perturbed by external tones: Response to a single tone at multiple suppression levels. William J. Murphy (Bioacoust. and Occup. Vib. Sect., Natl. Inst. for Occup. Safety and Health, MS C-27, 4676 Columbia Pkwy., Cincinnati, OH 45226-1998), Arnold Tubis, and Carrick L. Talmadge (Purdue Univ., West Lafayette, IN 47907-1396)

The relaxation dynamics of spontaneous otoacoustic emissions (SOAEs) interacting with an external tone have been successfully described using a Van der Pol limit-cycle oscillator model [Murphy et al., J. Acoust. Soc. Am. 97, 3702-3711 (1995a) and Murphy et al., J. Acoust. Soc. Am. 97, 3712-3720 (1995b)]. The theory developed in Murphy et al. (1995a) includes the possibility for multiple external tones to produce suppression of the oscillator amplitude. The relaxation dynamics are dependent upon the final state of the oscillator. The oscillator's rate of transition between two suppressed states is a function of $\kappa_{2}^{2} r_{1}$, the final amount of suppression and the negative damping parameter $r_{1}$. Data were collected from an SOAE interacting with a single frequency ipsilateral suppressor. Transitions between different suppressed states were achieved by adding or removing signal at the same frequency. The ability of the relaxation dynamics theory to describe this special case will be examined.
4aPP2. Effects of repetition rate, phase, and frequency on the auditory brain-stem response in newborn neonates and adults. $\mathrm{T}$. K. Parthasarathy (Dept. of Speech Pathol. and Audiol., Southern Illinois Univ., Edwardsville, IL 62026), Paul Borgsmiller (Cardinal Glennon Children's Hospital, St. Louis, MO 63104-1095), and Barbara Cohlan (St. Mary's Health Ctr., St. Louis, MO 63117)

Effects of repetition rate, phase, and frequency on wave $V$ latency of the auditory brain-stem response (ABR) were evaluated and compared in ten normal newborn neonates and adult subjects. Single cycle sinusoids at 0.25 and $2.0 \mathrm{kHz}$ were presented monaurally via an insert earphone at 75 $\mathrm{dB} n H L$, using either a condensation (C) or a rarefaction ( $R$ ) stimuli at two rates, 11.1 and $55.5 / \mathrm{s}$. The stimulus phase by frequency interaction was significant. In both neonates and adult subjects, the ABR wave V latency for R stimuli was significantly shorter compared to $C$ stimuli at $0.25 \mathrm{kHz}$. However, the effect of phase on wave $\mathrm{V}$ latency was insignificant at 2.0 $\mathrm{kHz}$. Furthermore, the stimulus rate by age interaction was significant. The $A B R$ wave $V$ latency shift with an increase in repetition rate was significantly greater in the newbom neonates than in the adult subjects. 
4aPP3. Endolymph volume regulation mechanisms revealed by microinjections into scala media. Alec N. Salt and John E. DeMott (Dept. of Otolaryngol., Washington Univ. School of Medicine, $517 \mathrm{~S}$. Euclid Ave., St. Louis, MO 63110)

Longitudinal movements of endolymph were quantified in vivo in the basal turn of the guinea pig cochlea during injections of artificial endolymph into the second tum at rates up to $70 \mathrm{nl} / \mathrm{min}$. Flow measurements utilized the marker ion tertramethylammonium (TMA), which was iontophoretically injected into the basal turn. TMA dispersion was measured by two ion-selective microelectrodes, one placed $0.5 \mathrm{~mm}$ apical, and the other $0.5 \mathrm{~mm}$ basal to the TMA injection site. Longitudinal endolymph movements were calculated from the recorded TMA time courses. Prior to injections, endolymph flow rates were extremely low. Volume injection into the second turn induced basally directed flows in the basal turn. The relationship between the measured rate and the injection rate was nonlinear, in which low-injection rates produced proportionately less flow than higher rates. These data show that for injections at low rates, volume disturbances have primarily local effects, presumably generating local distension or being compensated by local homeostatic mechanisms. At higher rates, basally directed endolymph flow becomes increasingly significant. These findings suggest that two independent processes may be involved in the regulation of endolymph volume. [Work supported by NIH Grant No. DC01368.]

4aPP4. Directionality of sound-pressure transformation at the pinna of Mus domesticus. Qi-Cai Chen (Dept. of Biology, Central China Normal Univ., Wuhan, Hubei, People's Republic of China), David Cain, and Philip H.-S. Jen (Univ. of Missouri, Columbia, MO 65211)

Sound-pressure transformation properties at the pinna of laboratory mice, Mus domesticus, were studied by measuring the sound-pressure level of a continuous tone at a series of frequencies at the tympanic membrane as a function of the position of a sound source under free-field stimulation conditions. The spectral transformation, the interaural spectral difference, the isopressure contours and the interaural pressure difference contours were plotted, Sound-pressure transformation functions showed some prominent spectral notches throughout the frequency range of $10-80 \mathrm{kHz}$ tested. When delivered from some angles within the ipsilateral frontal hemisphere, the sound pressure at the tympanic membrane of certain frequencies may be lower than that determined at the corresponding contralateral angles (i.e., the negative pressure). For each sound frequency tested, there was an angle (the acoustic axis) within the ipsilateral frontal hemisphere from which the delivered sound reached a maximal pressure level at the tympanic membrane. However, the acoustic axis often changed to a new angle after removal of the ipsilateral pinna. Removal of the ipsilateral pinna or modification of pinna posture expanded isopressure contours irregularly and split the 2-dB maximal pressure area into several parts. [Work supported by NIH and HFSP.]

4aPP5. The influence of sound direction on frequency tuning of inferior collicular neurons of the big brown bat, Eptesicus fuscus. Yi-Wen Chen, Ka-Choi Tang, and Philip H.-S. Jen (Div. of Biological Sciences, Univ. of Missouri, Columbia, MO 65211)

The influence of sound direction on frequency tuning of 64 inferior collicular neurons of the big brown bat was studied by comparing the best frequency and $Q_{10-\mathrm{dB}}, Q_{30-\mathrm{dB}}$, and $Q_{50-\mathrm{dB}}$ values of each neuron determined from five sound directions between $\pm 70^{\circ}$ in azimuth. Sound direction did not affect the BF of all but three neurons by more than $5 \mathrm{kHz}$ but it affected at least one $Q$ value of $51(80 \%)$ neurons by more than $50 \%$. Whereas sound direction affected all three $Q$ values of $9(14 \%)$ neurons, it afected no more than two $Q$ values of the remaining $42(66 \%)$ neurons. Sound direction also affected the shape of frequency tuning curves in 21 $(32.8 \%)$ neurons. Frequency tuning of 30 collicular neurons to different sound directions was also studied before and after ionophoretical application of bicuculline to each recorded neuron. Bicuculline application not only lowered the minimum threshold of $20(67 \%)$ neurons in all sound directions, it also shifted the lowest MT of $10(33 \%)$ neurons to a different sound direction. In addition, it reduced the $Q_{10-\mathrm{dB}}$ values of $18(60 \%)$ neurons and changed the shape of the frequency tuning curves of 21 (70\%) neurons.
4aPP6. An evoked potential study of directional sensitivity in the inferior colliculus of the laboratory mouse (Mus domesticus). David M. Cain and Philip H.-S. Jen (Div. of Biological Sciences, Univ. of Missouri, Columbia, MO 65211)

Directional hearing of the laboratory mouse was studied by recording 30 evoked potential responses from the inferior colliculus to best frequency (BF) sounds delivered from within the frontal auditory field. Audiograms determined with maximal response amplitude $(N=6)$ and minimum threshold $(N=8)$ showed maximal auditory sensitivity occurring between $10-15 \mathrm{kHz}(M \pm$ s.d. $=11.2 \mathrm{kHz} \pm 2.34)$. For five evoked potentials, amplitude-intensity functions were obtained for seven selected azimuthal angles at $0^{\circ}$ elevation. All 35 functions were nonmonotonic but their dynamic range was affected by sound direction. The azimuthal and elevational angles of maximal auditory sensitivity (the response centers) of 25 evoked potentials responses, were always located in the upper, contralateral quadrant $\left(M \pm\right.$ s.d. $=$ up $25^{\circ} \pm 19.97$, contralateral $39^{\circ} \pm 11.73$ ). Spatial response areas measured at either 3 or $5 \mathrm{~dB}$ above the minimum threshold decreased with stimulus frequency $(3-\mathrm{dB}$ area: $r=0.53$, $p=0.0118$, and 5-dB area: $r=0.44, p=0.0338$ ). Spatial response areas associated with higher BFs were more concentric and smaller than those of lower BFs, which were larger and often expanded irregularly beyond the tested angles of the frontal auditory field. The results reflect the directionality of the sound-pressure transformation at the pinna of the mouse as demonstrated in our recent study (Chen et al., in press).

4aPP7. Discharge patterns and amplitude modulation encoding in the torus semicircularis of the frog. Nikolay G. Bibikov (Acoust. Inst., Schvernik st., 4, Moscow 117036, Russia)

Responses to pure and sinusoidally $20-\mathrm{Hz}$ amplitude-modulated tone bursts $(612,5$-ms stimulus on time at the rate of once per $2.2 \mathrm{~s})$ were recorded in 202 units of the torus semicircularis (midbrain auditory center) in the immobilized grass frogs (Rana temporaria). Best frequency tones at $30 \mathrm{~dB}$ re: minimum threshold were used. The largest values of synchronization coefficients (SCs) to $80 \%$ amplitude-modulated stimuli were observed for same phasic units. The proportion of the responses with a good phase-locking ( SC >0.5) was, however, higher in the tonic unit's population. Only in a few phasic units was the sustained response to $10 \%$ modulated stimuli observed. These particular units demonstrated, however, extremely high SCs. On the other hand, the phase-locking response to tones at $10 \%$ modulation depth was observed in $65 \%$ of the tonic units. In the majority of these neurons the partial SCs for successive modulation periods increased considerably from the initial to the terminal modulation periods. The psychophysical correlates of this physiological "overshoot" effect are discussed. [Work supported by ISF and RFFI.]

4aPP8. Correlations between auditory-filter shape parameters measured at proximal center frequencies. Marc A. Fagelson (Program in Comm. Sci. \& Disord., Univ. of Texas, Austin, TX 78712) and Craig A. Champlin (Univ. of Texas, Austin, TX 78712)

Auditory-filter shape parameters in 20 normal-hearing listeners were determined at center frequencies (CFs) of $913,1095,3651$, and $4382 \mathrm{~Hz}$ using the five-point roex $(p, r)$ method. Slopes of the filters' skirts were correlated for the CFs in each frequency region at both low and high stimulus levels. In the $\lambda=1000-\mathrm{Hz}$ region, the auditory filters' lowfrequency slopes were significantly correlated at the low and high stimulus levels, while the high-frequency slopes were associated at the high, but not the low level. In the $\lambda=4000-\mathrm{Hz}$ region the relationships were clearer as the low-frequency and high-frequency filter skirts diverged at the low level, but were significantly correlated at high stimulus levels. Level dependencies in auditory-filter shapes indicated subtle differences in cochlear frequency analysis at proximal places along the basilar membrane diminished as signal level was increased. Conversely, in those situations most likely to be affected by active processing along the partition, such as the low-level and high-frequency conditions, auditory filters centered at neighboring frequencies often did not resemble one another. This suggests that active cochlear mechanisms are not uniformly distributed throughout the length of the basilar membrane. [Work supported by the College of Communication (Jamail Grant) and NIDCD.] 
4aPP9. Source spectrum recovery at different spatial locations. Ewan A. Macpherson (Waisman Ctr., Univ. of Wisconsin-Madison, 1500 Highland Ave., Madison, WI 53705-2280)

The directional filtering of sound by the pinnae is vital to localization, but distorts the spectrum of the signal reaching the eardrum. Sounds do not appear to change character dramatically as a function of direction, so listeners might have some ability to deconvolve pinna effects from the received signal. A three-interval profile analysis task measured listeners' ability to recover source spectra. The stimuli were wideband noise bursts in which the levels of $1 / 3$-octave bands were adjusted to control spectral shape. They were filtered by listeners' measured head-related transfer functions (HRTFs) and delivered via headphones. Listeners discriminated between various nonflat spectra and perturbed versions of them. HRTFs were selected randomly for each interval, and localizability was controlled by varying the correspondence of the left and right HRTFs. Thresholds were highest when HRTFs were imposed diotically, and lowest in an unfiltered diotic baseline condition. Accurate spatialization improved recovery, but applying independent near-ear HRTFs dichotically was more effective. However, deconvolution was imperfect in both conditions. The results suggest that the ability to disregard HRTF filtering and achieve some timbre constancy over direction depends on having two independent, wideband "looks" at the source spectrum, rather than on accurate localization.

4aPP10. The role of temporal factors in pitch perception. Valter Ciocca (Dept. of Speech \& Hearing Sciences, Univ. of Hong Kong, 34 Hospital Rd., Hong Kong)

This study investigated how pitch perception mechanisms integrate acoustic information over time. The pitch matching procedure developed by Moore et al. [J. Acoust. Soc. Am. 77, 1853-1860 (1985)] was used in order to measure pitch shifts in a harmonic series (target complex) produced by mistuning a harmonic that either preceded or followed the target complex. In the first experiment, the mistuned component could either stop as the target complex started (pretarget condition) or start as the target stopped (post-target condition). The results showed that pitch shifts were significantly larger in the post-target than in the pretarget condition. In the second experiment, the duration of the silent period, which separated the mistuned component and the target complex, was varied in both the preand the post-target conditions. Pitch shifts were virtually eliminated by a delay longer than $20 \mathrm{~ms}$ in the pretarget condition. By contrast, a delay of $160 \mathrm{~ms}$ was necessary to eliminate pitch shifts in the post-target condition. These results suggest that pitch perception mechanisms take into account the order of occurrence of acoustic information for calculating the pitch of a complex sound. [Work supported by Hong Kong RGC, Grant HKU $362 / 94 \mathrm{M}$.]

4aPP11. Glide difference limens as a function of center frequency, duration, and transition size. John P. Madden (Dept. of Commun. Disord., Univ. of North Dakota, University Station, Grand Forks, ND 58202-8040)

The study determined glide difference limens for up and down glides in several conditions. Signal durations were 50,100 , and $400 \mathrm{~ms}$, and the average stimulus center frequencies were 2 and $6 \mathrm{kHz}$. The standard, or comparison, signals changed in frequency by 0,500 , and $1000 \mathrm{~Hz}$. These transition sizes were chosen to cover the range of $F 2$ formant transitions. The subject's task was to distinguish between the standard and a target signal with a greater or lesser change in frequency, depending on the experimental condition, in a 2-alternative, forced-choice task. To avoid the confounding effect of between-stimuli static pitch cues, the center frequencies of the standard and target stimuli were "roved" about the center frequencies [Neill and Feth, J. Acoust. Soc. Am. Suppl. 1 87, S23 (1990)]: The data were analyzed using a level-detection model consisting of a filter bank, a nonlinearity, a temporal integrator, and a detection device. The question of whether the results support a rate place or temporal mechanism of frequency coding is discussed. [Work supported by NIDCD.]

\title{
Session 4aSA
}

\section{Structural Acoustics and Vibration: Statistical Methods in Complex Structures I}

\author{
Richard L. Weaver, Chair \\ Department of Theoretical and Applied Mechanics, University of Illinois, 104 Wright Street, Urbana, Illinois 61801
}

Invited Papers

4aSA1. Parameter estimation for fuzzy structures. A result concerning vibrations in the low-frequency range. Christian Soize (Structures Dept., ONERA, BP 72, 92322 Chatillon Cedex, France)

In the field of structural vibrations, the structural complexity can be induced by "secondary" mechanical subsystems attached to the "master" structure or by "local eigenmodes" of some continuum elastic subelements of the master structure; these local eigenmodes induce a structural complexity when the model of these subelements can only restitute the elastostatic behavior but not its elastodynamic response. Within this context, a model is presented of the apparent vibration damping of the master structure due to the vibrations of the structural complexity. This vibration-damping model is deduced from the theory of fuzzy structures that was previously developed by the author. Presently, this model uses only the mean part of the probabilistic fuzzy law of the fuzzy substructure. A model of the generalized damping matrix deduced from the model of the structural complexity, is explicitly constructed. This generalized damping matrix depends on parameters related to the fuzzy substructure. Problems related to the model parameters estimation are studied. Finally, an example is presented and allows the theory to be validated.

\section{9:00}

4aSA2. Fuzzy elements, their coupling rules, and the Jaynes-Shannon maximum entropy principle. Allan D. Pierce (Boston Univ., Dept. of Aerospace and Mech. Eng., 110 Cummington St., Boston, MA 02215)

Emerging theories of fuzzy structures are regarded as the wholesale replacement of certain portions of the structure by fuzzy elements, whose chief characteristic is a smeared-out (fuzzied) distribution of natural frequencies, so that there are an infinite number of natural frequencies in any given frequency band. Descriptors of fuzzy elements are the mass per unit natural frequency band and 
the elements of a mass marrix per unit natural frequency band. Modeling of complex structures is simplified by regarding internal and appended structures as assemblages of coupled fuzzy elements, so circuit laws are here suggested for determining parameters of an overall fuzzy element from the known features of its fuzzy components. In regard to assigning mass distributions among frequencies to actual elements that are to be modeled as fuzzy elements, the appropriate guide is Jaynes's (1957) general method of statistical inference (principle of maximum entropy) extracted from Shannon's information theory. The mass distribution is regarded as a probability distribution, and the known features, such as total mass, asymptotic behavior, mass-weighted average natural frequency, d-c behavior, are imposed as constraints on a variational principle that maximizes the entropy asssociated with that probability distribution, to find the most likely such distribution consistent with the known information. [Work supported by Office of Naval Research.]

9:30

4aSA3. Real and apparent dissipation of vibrations of structural systems. J. J. McCoy (School of Eng., Catholic Univ. of America, Rm. 102, Pangborn Hall, Washington. DC 20064)

Discussions of "real" and "apparent" dissipation of the vibrations of structural elements to which are attached substuctures, have suffered from a lack of precision in terminology. Identifying a real dissipation with the transformation of mechanical energy to heat and an apparent dissipation with the transformation of mechanical energy from a form that one observes to a form that one doesn't. would clarify issues. A further classification of a real dissipation as either "resonant" or "nonresonant" is suggested. A resonant dissipation is obtained for vibration frequencies that are nearly coincident with the natural frequencies of a subset of the attached subsystems. Finally, a further classification of an apparent dissipation as either "reversible" or "irreversible" is also suggested. The physics underlying the different type dissipations; their modeling; and, the dependence of measures of dissipation on more fundamental measures of the attached substructures will be discussed.

10:00

4aSA4. Time domain simulation and visualization of fuzzy structures. Victor W. Sparrow (Graduate Prog. Acoust., Penn State Univ., 157 Hammond Bldg., University Park, PA 16802)

Fuzzy structure formulations divide a complicated structure into a well-known master structure and an imprecisely known fuzzy substructure. The loss of energy from the master structure, now known in the fuzzy structures literature as apparent damping, is simply a transfer of energy into the internal degrees of freedom of the attached fuzzy substructure. Some energy in the substructure is not returned to the master structure if either the internal damping of the substructure is non-negligible or there are a large number of degrees of freedom in the substructure with closely spaced resonances. This paper will demonstrate such effects using transverse wave pulses propagating on simple master structures, strings, and rods. Sets of one degree of freedom (dof) oscillators, drawn from particular statistical distributions, are attached as the fuzzy substructure. Finite-difference models for the strings and rods are solved simultaneously with the dynamics of the attached one dof oscillators in the time domain. Videotape animations show both the displacements of the master structure and the attached oscillators. Quantitative comparisons are made to the recent results of Russell [J. Acoust. Soc. Am. 97, 3414-3415(A) (1995)]. [Work supporled by ONR.]

10:30-10:45 Break

\section{Contributed Papers}

\section{$10: 45$}

4aSA5. Analysis of a model structural fuzzy with long-range spatial interactions. Melvin E. King (Dept. of Aerospace and Mech. Eng., Boston Univ., 110 Cummington St., Boston, MA 02215)

Considerable progress has recently been made in analyzing the vibrational response of systems that enclose complex internal structures by introducing the notion of structural fuzzies. For globally distributed fuzzies that locally interact with a master structure (i.e., systems without length scales), a primary descriptor for the structural fuzzy has been identified as the density of mass per unit temporal frequency, $d M / d \omega$. Many practical structures, however, possess internals which are not local in nature, and therefore couple with the response of a master structure over various length scales. In an effort to address such problems, a prototypical model has been selected which simulates a network of pipes, ducts, and/or conduits attached to a master structure. The model consists of a flat plate (taken to be the master structure) onto which is attached a network of pipes (taken to be the spatially interacting structural fuzzy). Each pipe is attached to the plate via periodically spaced supports (assumed to be rigid and massless), and only coupled transverse motions are considered. Using this model system, basic insights into frequency wave-number interactions due to the structural fuzzy are sought and important physical parameters are identified.

\section{1:00}

4aSA6. Determination of modal masses from plots of driving point impedance magnitude versus frequency when modal overlap is present. Jacques Livingston and Allan D. Pierce (Boston Univ., Dept. of Aerospace and Mech. Eng., 110 Cummington St., Boston, MA 02215)

Implementation of a piecemeal technique for the modeling of complex structures within the context of the theory of fuzzy structures requires that one have some prior knowledge of the distribution of modal masses for each class of intemal appendages that have similar design features and function. For each such category, sample calculations or measurements can yield data that can be gathered into an ensemble and then used in the computational replacement of each appendage within that category by a generic fuzzy element. The present paper is concerned with how such data might be obtained from experimental tests that determine how the magnitude squared of the driving point impedance varies with frequency. For a lightly damped one-degree-of-freedom system, basic vibration theory leads to an expression for the (modal) mass which is proportional to the height of the resonance peak divided by the square root of the magnitude of the second derivative at that peak. However, for a multidegree-of-freedom or continuum structure, a fundamental scheme based on the measurement of heights of peaks and of the second derivatives at such peaks can be frustrated by the occurrence of strong modal overlap. A numerical iteration method that can largely overcome this makes use of a general functional 
form for the complex impedance and proceeds to gradually "filter out" the contributions from the stronger peaks, so that the weaker peaks can be identified and then also filtered out. [Work supported by Office of Naval Research.]

\section{1:15}

4aSA7. Nonlinear localization in mistuned periodic structures. Philip Layne and Melvin E. King (Dept. of Aerospace and Mech. Eng., Boston Univ., 110 Cummington St., Boston, MA 02215)

A characteristic phenomenon encountered in weakly coupled repetitive systems (systems composed of identical, reperitive substructural elements) is mode localization. Such localized modes have been investigated widely in the literature, in which it has been shown that eigenvalue veering in mistuned linear systems and mode bifurcations in perfectly tuned nonlinear systems give rise to motions during which a system's vibrational energy may be spatially confined to a small subset of its elements. In the present work, a systematic investigation of the combined effects of nonlinearities and structural mistunings is discussed. The method of multiple scales is utilized to compute localized modes for an $\boldsymbol{n}$ degree-of-freedom nonlinear cyclic system with structural mistunings. Strong and weak localized motions are computed for various structural parameters, and it is shown that the presence of nonlinearities and mistunings can enhance the localization effect for some modes, while simultaneously diminishing the effect for others. Sample calculations will be presented for systems composed of two, three, and four degrees of freedom. The implications of nonlinear mode localization for vibration isolation are also discussed.

\section{1:30}

4aSA8. Anomalous attenuation in disordered networks. J. Dickey, G. Maidanik (David Taylor Res. Ctr., Annapolis, MD 21402), and J. D'Archangelo (U.S. Naval Acad., Annapolis, MD 21402)

A two-dimensional "jungle gym" is modeled as a network of connected dynamic systems, each characterized by a propagation wave number, loss factor, and length. The response of the network to an out-of-plane harmonic drive is calculated as a function of frequency. When the systems are all identical (i.e., a regular lattice) the modes are well defined and identifiable as being either modes of the individual systems or global modes of the network. Further, the pass and stop band structure in the network is distinct. When the system lengths are not regular, and in particular when they are randomly distributed, the modes are not easy to classify and the transmission of waves through the network is profoundly affected. This tends to destroy both pass and stop bands giving rise to an attenuation which is an average of the pass and stop bands, i.e., an "amber" band (since pass bands are green, stop bands are red and amber bands lie in between). In other words, perturbing the lengths gives rise to a structure whose attenuation (and transmission) from one point to another far exceeds that which would be expected based on the propagation loss of the constituent systems.

\section{1:45}

4aSA9. Loss factor of a beaded structure. G. Maidanik and J. Dickey (David Taylor Res. Ctr., Bethesda, MD 20084)

It is found that when beads are inserted into the interior of a pipelike structure, the damping of the beaded structure may substantially exceed that of the unbeaded structure. The beads are modeled by a "beaded fluid" characterized by a low density, a low sound speed, and a high loss factor. Statistical energy analysis (SEA) is developed to account for the presence of the beaded fluid within the structure. It is shown that in certain frequency regimes and certain choices of the parameters that characterize the beaded fluid, relative to those that characterze the structure, the loss factor of the beaded structure can be designed to significantly exceed that of the unbeaded structure. The mechanism for the increase lies in that the stored energy in the beaded fluid may, in the "steady state," be made to substantially exceed that in the structure. The bulk of the stored energy is then decimated by the high damping capability that the beaded fluid enjoys. A selected number of computer experiments are cited to illustrate the potential effectiveness of this damping mechanism and the manner by which the parameters, that define the beads and the structure, control it.

THURSDAY MORNING, 30 NOVEMBER 1995

ROSE GARDEN, 8:00 TO 11:45 A.M.

Session 4aSC

\section{Speech Communication: Current Directions in Vowel Perception Research}

Peter F. Assmann, Cochair

School of Human Development. University of Texas at Dallas, Box 830688, GR 4.1, Richardson, Texas 75083

Randy L. Diehl, Cochair

Department of Psychology, University of Texas, 300 Mezes, Austin, Texas 78712

Chair's Introduction-8:00

Invited Papers

8:05

4aSC1. Vowel formant discrimination. Diane Kewley-Port (Dept. of Speech and Hearing Sciences, Indiana Univ., Bloomington, IN 47405)

This series of experiments examines the relation between the basic capabilities of the peripheral auditory system to process speech and the classification of speech at more central levels. Psychophysical techniques have been applied to determine listeners' abilities to detect, discriminate, and identify speech sounds. The current series of experiments established that thresholds for the discrimination of vowel formants are constant in the $F 1$ region with a value of $F=14 \mathrm{~Hz}$ and in the $F 2$ region increase linearly with increasing frequency. This presentation describes three directions of our recent research. First, the effects of various stimulus parameters, including fundamental frequency and consonantal context, on thresholds were explored. Second, excitation pattern models have been 
shown to account for the significant effects of vowel formant frequency and fundamental frequency on $F$ thresholds. Third, an initial experiment compared formant discrimination to vowel identification for listeners with normal hearing and moderate hearing impairment. Results demonstrated that for the impaired listeners, reduced ability to identify vowels was partially predicted by elevated discrimination thresholds in the $F 2$ region. [Work supported by NIDCD.]

8:30 Commentary by Robert A. Fox

Speech and Hearing Science, Ohio State Univ., Columbus, OH 43210-1372

8:45 Discussion

8:55

4aSC2. Vowel recognition: Formants, spectral peaks, and spectral shape. James Hillenbrand (Speech Pathol. and Audiol., Western Michigan Univ., Kalamazoo, MI 49008) and Robert A. Houde (RIT Res. Corp., Rochester, NY 14623)

The purpose of this presentation will be to review some recent research on the acoustic characteristics of American English vowels, and to discuss some issues related to the auditory mechanisms underlying vowel recognition. Evidence from studies using traditional formant frequency representations will be reviewed to address issues such as talker normalization and the role of dynamic features in vowel identification. A long-standing debate in phonetic perception theory concerning whether phonetic quality is controlled by formant frequencies or the overall shape of the spectrum will also be addressed. While formant theory has tended to dominate much of vowel perception research, some compelling arguments have been leveled against formant representations; however, there are also some very important problems with whole-spectrum representations. A new method of representing speech will be described which is believed to address some of the limitations of both formants and overall spectral shape. The masked peak representation (MPR) was designed to: (1) show maximal sensitivity to spectral peaks, (2) show minimal sensitivity to spectral shape details in nonpeak regions, and (3) avoid explicit formant tracking. Preliminary results will be described from an experiment in which listeners are asked to identify $/ \mathrm{hVd}$ utterances that were synthesized from MPR spectra.

9:20 Commentary by Stephen A. Zahorian

Electrical and Computer Engineering. Old Dominion Univ., Norfolk. VA 23508

9:35 Discussion

9:45-10:00 Break

10:00

4aSC3. Acoustic correlates of perceived vowels of American English. James D. Miller (Central Inst. for the Deaf, 818 S. Euclid, St. Louis, MO 63110)

Relations between four acoustic paramenters, fundamental frequency $(F 0)$ and the center frequencies of the first three formants $(F 1, F 2$, and $F 3)$, and the perception of vowels are described. Prediction of listeners' identifications of vowels are best when acoustic trajectories are based on all four parameters. These parameters can be taken separately to form a four-dimensional space or they can be combined to form a three-dimensional space such as Miller's Auditory Perceptual Space (APS). The time-normalized paths through such spaces correlate best with listener responses. Temporal factors such as durations and speeds along these paths, within limits, are not critical. However, the direction of movement along the path can be crucial. While movement in a forward direction usually evokes the perception of the intended vowel, the opposite movement may sometimes evoke the perception of another vowel. Recent work shows that neural networks, trained with inputs based on $F 0, F 1, F 2$, and $F 3$, perform very similarly to humans listening to the waveforms of the isolated nuclei. These results will be reviewed and their implications for models of vowel perception will be discussed. [Work supported by NIDCD, AFOSR, and CID.]

10:25 Commentary by Winifred Strange

Communication Sciences. Univ. of South Florida. Tampa, FL 33620

10:40 Discussion

10:50

4aSC4. Talker variability in vowel perception. Keith Johnson (Dept. of Linguist.. Ohio State Univ., 222 Oxley Hall, 1712 Neil Ave.. Columbus, OH 43210-1292)

Individual differences in the speech acoustic waveform create complications for theories of human speech perception, and auditory word recognition, as well as for automatic computer speech recognition systems. Theories of vowel perception, often taking the form of scatter reduction techniques more or less related to possible auditory or cognitive mechanisms, have been proposed to deal with individual differences. Word recognition theorists are also beginning to grapple with the problem of talker variability and implicit memory for talker-specific acoustic patterns. Talker variability is also one of the central problems in automatic computer speech recognition, where the dichotomy between speaker-dependent and speaker-independent systems has recently been augnented by new hybrid speaker-adaptive systems. Recently it has become apparent that all three of these areas of research are converging on the same 
conclusion - that speaker variability is best handled by rich, speaker-specific representations. In speech perception, this viewpoint has been called "indirect" speaker normalization. In the theory of word recognition, exemplar-based models of memory have been proposed to account for talker variability, and in automatic speech recognition a similar strategy is evident in multimodel systems and data augmentation approaches to speaker adaptation.

11:15 Commentary by Terrance $M$. Nearey

Linguistics Dept., Univ. of Alberta, Edmonton, $A B$ T6G 2E7, Canada

11:30 Discussion

THURSDAY MORNING, 30 NOVEMBER 1995

ST. LOUIS C, 9:00 TO 11:30 A.M.

Session 4aSP

Signal Processing in Acoustics: Higher-Order Spectra and Trans-Spectra

Gary R. Wilson, Chair

Applied Research Laboratories, P.O. Box 8029, Austin, Texas 78713-8029

Invited Papers

9:00

4aSP1. Quantification of nonlinear phenomena in laboratory-generated random sea waves. Edward J. Powers, Sungbin Im, In-Seung Park (Dept. of Elec. and Comput. Eng. and Offshore Technol. Res. Ctr., Univ. of Texas, Austin, TX 78712-1084), Arun Duggal, and Peter Johnson (Texas A \& M Univ., College Station, TX 77843-3400)

In this paper higher-order statistical signal processing and Volterra series models of nonlinear phenomena are utilized to quantify the role of nonlinear second-order effects in laboratory-generated random seas. It is indicated how higher-order moment spectra may be utilized to determine a frequency-domain Volterra model of the linear and second-order wave physics occurring between two closely spaced points at which the random wave field is sampled. It will be shown that although the amount of "energy" associated with second-order effects is very small, such second-order phenomena play an important role in the generation of large amplitude waves. By inverse Fourier transforming the outputs of the linear and quadratic Volterra filters, it is demonstrated that the generation of large amplitude waves is due to momentary phase locking of the first- and second-order components. All of the phenomena mentioned above will be demonstrated with the aid of experimental data collected at the Offshore Technology Research Center's Model Basin. [This study was supported by the National Science Foundation Engineering Research Centers Program Grant Number CDR-8721512 through the Offshore Technology Research Center (OTRC). The Volterra modeling techniques were developed under ONR Grant N00014-92-J-1046 and the Joint Services Electronics Program AFOSR F-49620-92-C-0027.]

9:30

4aSP2. Advanced acoustic signal detection and localization with lower-order statkstics. Panagiotis Tsakalides and Chrysostomos L. Nikias (Signal and Image Processing Inst., Dept. of Elec. Eng.-Systems, Univ. of Southern California, 3740 McClintock Ave., Rm. 400B, Los Angeles, CA 90089-2564)

The importance of extending the statistical signal processing methodology to the so-called alpha-stable farmework is apparent. First, scientists and engineers have started to appreciate alpha-spectra and the elegant scaling and self-similarity properties of stable distributions. Additionally, real life sonar applications exist in which impulsive ocean channels tend to produce large-amplitude, short-duration interferences more frequently than Gaussian channels do. The stable law has been shown to successfully model noise over certain impulsive channels. In this lecture, new robust techniques are proposed for source detection and localization in the presence of noise modeled as a complex isotropic stable process. First, optimal, in the maximum likelihood sense, approaches are presented and the Cauchy beamformer is introduced. Also, subspace methods based on fractional lower-order statistics are developed for sonar applications where reduced computational cost is a crucial design parameter. Finally, simulation experiments demonstrating the performance of the proposed methods are presented. 
4aSP3. Multilag high-order ambiguity function for multicomponent polynomial phase signals. Georgios B. Giannakis and Sergio Barbarossa (Dept. of Elec. Eng., Univ. of Virginia, Charlottesville, VA 22903-2442)

The analysis of polynomial phase signals using the recently developed polynomial phase transform suffers from spurious harmonics when dealing with multiple components having identical high-order coefficients. It is illustrated that such a model appears in synthetic aperture radars (SAR) imagery and transmission of polynomial phase signals through multipath channels. Analytical expressions for the spurious peaks are derived first, followed by algorithms for estimating the parameters of multicomponent polynomial phase signals taking advantage of the overdeterminacy introduced by the so called multilag high-order ambiguity function. An algorithm will be presented based on the projection of the observed signal onto a subspace estimated as the intersection of signal subspaces obtained using different sets of lags. Fast suboptimal algorthms will also be presented for suppressing interference terms and removing ambiguities. Autofocusing of real SAR imagery and mitigation of multipath when acoustic signals arrive at moving receivers, will be used to illustrate the usefulness of the proposed techniques.

10:30

4aSP4. Applications of the trans-spectral coherence technique. P. G. Vaidya (MME Dept., Washington State Univ., Pullman, WA 99164)

The trans-spectral coherence technique (TSC) [Vaidya and Anderson, J. Acoust. Soc. Am. 89, 2370-2378 (1991)] has been used to remove noise and distortions from periodic signals. Recent results show that the technique is also well suited to analyzing nonstationary signals, including chaos. For example, TSC can be used to distinguish a chaotic signal from a random one. As opposed to a truly random signal, in chaotic signals nonzero TSCs are required, at least for some combinations. In fact, for the Duffing equation undergoing chaos, nearly perfect trans-spectral coherence was observed for many combinations. In this case, TSC proved to be a very useful diagnostic tool, and it helped develop a deeper insight into the nature of the specific attractor, and the associated invariant group structures. Similar strong coherences have also been observed in speech signals. This sheds further light on the issues of speaker and speech recognition. Further, it is important in many fields (heart arrythmia, for example), to be able to predict in advance that a periodic system is likely to become chaotic. TSC can be used to arrive at a premonition of such a forthcoming change.

\section{Contributed Papers}

11:00

4aSP5. Preliminary analysis of SWellEX-3 noise characteristics. Lisa A. Pflug, Pam M. Jackson (Naval Res. Lab., Code 7176, Stennis Space Center, MS 39529-5004), Juliette W. Ioup, and George E. loup (Univ. of New Orleans, New Orleans, LA 70148)

Higher-order moment analysis is performed for segments of data taken during the SWellEX-3 experiment, which was conducted off the Southern California coast in 1994. One SWellEX-3 objective was to provide a controlled data set for the purpose of improving understanding of detection in shallow water dominated by local shipping noise. Ambient noise and radar ship tracks were recorded for two full weekends over which the shipping character of the area changed dramatically. Second- and higher-order detection performance depends on the statistical character of the noise including the moment properties. As a prelude to higher-order detection evaluation, segments of data during times of light and heavy nearby shipping are analyzed for second- and higher-order moments, stationarity, and directionality. [UNO research supported by Office of Naval Research.]

\section{$11: 15$}

4aSP6. Sequential classification of moving objects from higher-order information. Roger F. Dwyer (Information Processing Branch, Naval Undersea Warfare Ctr., New London, CT 06320)

Objects in motion, due to the Doppler shift, modify their spectra and to a greater extent modify their higher-order spectra. Sequential classification methods have been developed to exploit higher-order spectral information in active sonar returns. To demonstrate the ability to sequentially classify moving objects from higher-order spectra, five spheres of different composition and velocity were tested. The sequential classifier correctly identified the sphere and its velocity based on higher-order information. A comparison of the sequential classifier's performance as a function of signal-to-noise ratio using higher-order information and second-order information will be presented. 


\title{
Session 4aUW
}

\section{Underwater Acoustics: Shallow Water Acoustics I}

\author{
Raymond J. Nagem, Chair \\ Department of Aerospace and Mechanical Engineering, Boston University, 110 Cummington Street, \\ Boston, Massachusetts 02215
}

\section{Contributed Papers}

\section{8:00}

4aUW1. Surf zone acoustic measurements from DUCK94. Ellen S. Livingston and Bruce H. Pasewark (Naval Res. Lab., Washington, DC 20375)

Low-frequency $(0-600 \mathrm{~Hz})$ acoustic measurements were taken on a 20-element bottom mounted horizontal line array in the surf zone of Duck, NC, during the DUCK94 exercise of Oct 1994. These measurements of breaking wave noise comprise acoustic array data uniquely taken so close to shore. The array was oriented endfire to shore, at $500 \mathrm{~m}$ offshore in $6 \mathrm{~m}$ of water. The beamformed array data provide a space-time-frequency decomposition of the surf zone noise. In particular, comparisons of noise levels from inshore and offshore in low surf conditions and moderate surf conditions are shown. Individual hydrophone data show frequency versus time versus level dependence and indicates a correlation between breaking wave period and noise levels.

\section{8:15}

4aUW2. Characteristics of seismoacoustic propagation in a near-shore environment. William S. Hodgkiss, Gerald L. D'Spain, and William A. Kuperman (Marine Physical Lab., Scripps Inst. of Oceanogr., La Jolla, CA 92093-0701)

MPL conducted the adaptive beach monitoring experiment, a nearshore, seismoacoustic experiment, over April-June 1995, at Camp Pendleton Marine Base north of San Diego, CA. Other participants included $\mathrm{NRaD} / \mathrm{NCCOSC}, \mathrm{NRL}, \mathrm{ARL} / \mathrm{PSU}$, and ARL/UT. The objectives were to examine the generation and propagation of seismoacoustic energy from both signals of interest and ambient noise sources on land and in the ocean, and the signal processing structures needed to separate signals from noise. To help quantify the propagation characteristics, a series of controlled source tows was performed. Tow tracks included along-shore tows (at approximately constant depth of $20 \mathrm{~m}$ ), upslope/downslope tows from the surf zone to $3.5 \mathrm{~km}$ offshore, and cross-slope runs. Four tones at 95,145 , 195 , and $370 \mathrm{~Hz}$ were simultaneously broadcast by the source and received by two nearly orthogonal, 120 -m-long, 64-element, horizontal bottom hydrophone arrays located $3.4 \mathrm{~km}$ offshore in $20-\mathrm{m}$ water. For tows along a bottom depth contour, the acoustic field at the higher frequencies shows the classical two- or three-mode interference pattern, whereas those in the upslope/downslope and cross-slope directions are more complicated. Horizontal plane-wave beamforming techniques are used to examine the horizontal refractive effects of cross-slope propagation. [Work supported by ONR, Code 32.]

\section{8:30}

4aUW3. Features of the seismoacoustic ambient noise field in a near-shore environment. Gerald L. D'Spain, William S. Hodgkiss, Lewis F. Berger, and William A. Kuperman (Marine Physical Lab., Scripps Inst. of Oceanogr., La Jolla, CA 92093-0701)

This paper presents the features of the 1- to 750-Hz ambient noise field recorded during MPL's adaptive beach monitoring experiment, a nearshore seismoacoustic experiment off the Camp Pendleton Marine Base north of San Diego, CA. Signal propagation characteristics in this environment are presented in a companion paper. The data were recorded by two nearly orthogonal, horizontal hydrophone arrays located $3.4 \mathrm{~km}$ offshore in 20-m water. The most predominant aspect is the sounds created by biologics, particularly night-time "cycling" choruses of fish. Ideas on the use of these sounds for determining the geoacoustic properties of the ocean bottom are discussed. Also present at night are strong lines at $85 \mathrm{~Hz}$ and its two higher harmonics, associated with water pumps on the marine base used to fill wells in the surrounding hills. A still-puzzling feature is the broadband pulses $(50-200 \mathrm{~Hz})$ that occur at a 7.5 -s repetitition period; they possibly also are of a biological nature. Fluctuations in the infrasonic band are associated with ocean surface wave propagation, thereby providing a means of determining the ocean surface wave directional spectrum. Finally, by judicious selection of data, noise generated by breaking surf can be measured. [Work supported by ONR, Code 32.]

\section{8:45}

4aUW4. Characterizing parameter landscapes for poroelastic media. Joseph F. Lingevitch and Michael D. Collins (Naval Res. Lab., Washington, DC 20375)

There is presently a great deal of interest in modeling ocean sediments as poroelastic media. One of the topics of particular interest is matchedfield inversion for sediment parameters. This paper will investigate the resolvability of poroelastic parameters in ocean sediments using acoustic sources and receivers located in the water column. The eigenvectors and eigenvalues of the covariance matrix of the gradient of the cost function indicate the most important underlying parameters as well as the degree of coupling between parameters [M. D. Collins and L. Fishman, "Efficient navigation of parameter landscapes," J. Acoust. Soc. Am. 98, 1637-1644 (1995)]. This information is useful for designing effective experiments for resolving the desired parameters. The information in the covariance matrix can be used to determine the most effective frequencies and array configuration and to optimize the performance of the inversion at each frequency. Wave-number integration and parabolic equation models are available for solving the forward problem [M. D. Collins, W. A. Kuperman, and W. L. Siegmann, "A parabolic equation for poro-elastic media," J. Acoust. Soc. Am. 98, 1645-1656 (1995)].

\section{9:00}

4aUw5. Determining sediment absorption using ambient noise in shallow water. Nicholas M. Carbone, Grant B. Deane, and Michael J. Buckingham (Marine Physical Lab.-0238, Scripps Inst. of Oceanogr., 9500 Gilman Dr., La Jolla, CA 92093-0238)

The ambient noise field in shallow water interacts strongly with the underlying seabed. As a consequence, the vertical directionality of the noise is largely determined from the characteristics of the seabed. In previous research, this property has been utilized to perform inversions for the compressional and shear wave speeds in hard seabeds. Soft seabeds predominate along continental shelf regions. In these cases, the absorption of compressional waves in the sediment is an important determinant of the noise field statistics which creates a measurable asymmetry in the vertical 
directionality. This asymmetry has been used as the basis of an inversion methodology, whereby the compressional wave absorption is estimated from measurements of the noise. The technique uses a broadband measurement from a vertically separated hydrophone pair at a fixed location. The ambient noise method yields an absorption estimate representing an average over range and depth. The technique represents a new means of estimating seabed absorption which leaves the sediment undisturbed. Absorption estimates obtained from inversions of ocean noise data taken above various soft seabeds will be presented. [Work supported by ONR.]

\section{9:15}

4aUW6. A numerical solution of the parabolic elastic wave equation. Raymond J. Nagem (Dept. of Aerospace and Mech. Eng., Boston Univ., 110 Cummington St., Boston, MA 02215), Ding Lee (Naval Undersea Warfare Ctr., New London, CT), and Gongquin Li (Univ. of New Orleans)

Based on the parabolic equation approximation, a set of equations has been developed for three-dimensional time-harmonic wave propagation in an elastic medium. The elastic equations for the scalar and vector potentials are written in a matrix form which is a direct counterpart to previous work on the scalar wave equation for a fluid medium. An ordinary differential equation (ODE) method in conjunction with a finite-difference scheme leads to a stable marching procedure. One feature of this approach is that every finite-difference discretization results in a tridiagonal system of equations; these equations can be solved efficiently by recursive formulas. This paper reports the computational results which are used to check (1) the stability of the marching scheme, and (2) the accuracy of the elastic model. Accuracy and validity are verified by comparing the numerical results of the finite-difference method with a far-field analytic solution in an unbounded medium.

\section{9:30}

4aUW7. Acoustic bottom penetration in a shallow water site from a parametric projector. Nicholas P. Chotiros, Adrienne M. Mautner (Appl. Res. Labs., Univ. of Texas, P.O. Box 8029, Austin, TX 78713-8029), Áge Kristensen, Oddbjom Bergem, and Ame Lovik (SACLANT Undersea Res. Ctr., 19026 La Spezia, Italy)

Acoustic penetration of a sandy ocean sediment by a narrow beam signal was investigated. A parametric source, the Simrad TOPAS, projected acoustic pulses at a shallow grazing angle into a sandy silt sediment in which a sparse 3-D array of acoustic sensors was buried, in a site off La Spezia, Italy. Although the experiment was conducted in shallow waters, the received signals were free of surface-bounce multipaths, due to the narrowness of sound beam, and excellent penetration was obtained. The collected signals were processed for direction and speed of the sediment acoustic waves. From a theoretical point of view, the medium is treated as a poroelastic solid governed by Biot's theory of acoustic propagation. Comparisons are made between theory and experiment. [Work supported by Office of Naval Research, Ocean Acoustics Program, Code 321.]

\section{9:45}

4aUW8. Shallow-water acoustic measurements in the southern Adriatic Sea. J. M. Berkson (Naval Res. Lab., Washington, DC 20375), R. D. Hollett, and M. Max (SACLANT Undersea Res. Ctr., 1-19138 La Spezia, Italy)

Shallow-water acoustic and geoacoustic measurements were made in the southern Adriatic Sea in May-June 1995 in combination with detailed oceanographic, geological, and geophysical surveys. Seismic subbotom profiling, narrow-beam echosounding, and bottom coring results were used to select two shallow water sites with downward refracting conditions for acoustic experiments: (1) a flat seafloor at $90-\mathrm{m}$ depth with about $1 \mathrm{~m}$ of rigid, nongaseous clay over a sequence of laminated clays and (2) a seafloor sloping from 49- to $37-\mathrm{m}$ depth and consisting of soft gaseous mud over laminated clay. Broadband sparker and 180-g TNT explosive sources were used for propagation and reverberation to a vertical array receiver in addition to cw sources for propagation. Initial results for the deeper water site show frequency-independent propagation that is consistent with modeled results using geoacoustic parameters extrapolated from coring results. A modification of the Dix method to estimate sediment sound speed versus depth from wide-angle reflectivity experiments made at the deeper water site is described. [Work supported by ONR.]

\section{0:00-10:15 Break}

\section{0:15}

4aUW9. Seismoacoustic modeling of thin sedimented regions in the mid-Atlantic ridge. Stanley A. Chin-Bing (Naval Res. Lab., Stennis Space Center, MS 39529-5004) and Joseph E. Murphy (Univ. of New Orleans, New Orleans, LA 70148)

In several previous meetings results have been reported comparing computer generated acoustic simulations with reverberation data taken from the mid-Atlantic ridge region during the 1993 Acoustics Experiment ("Site A, Seg 076" from the ONR Acoustic Reverberation Special Research Program). The acoustic simulations used representations of the rough, range-dependent ocean sediment/subbottom bathymetry that were generated by geomorphology models (the Webb-Jordan sediment distribution model and the Goff-Jordan fractal seafloor/basement model). A modified version of the Collins FEPE2WAY model was used for the longrange reverberation predictions. The simulated backscatter signal (210$280 \mathrm{~Hz}$ ) compared favorably, in structure and level, with the reverberation data. In this study the same PE model is used to propagate the incident field to a thin sedimented region of interest. This field is then used as the initial field to the FFRAME and SAFE models to study the seismoacoustic influence of the thin sedimented regions on reverberation. Results will be presented that indicate the effects that shear waves and shear wave attenuations have on the reverberation. [Work supported by Office of Naval Research, Acoustic Reverberation SRP, and a High Performance Computing DoD Shared Resource Center grant.]

\section{0:30}

4aUW10. Gaussian ray bundles for simulating shallow-water torpedo reverberation. Henry Weinberg (Naval Undersea Warfare Ctr. Detachment, New London, CT 06320-5594) and Ruth E. Keenan (Science Applications Intl. Corp., Mashpee, MA 02649)

A range-dependent propagation model was developed to simulate shallow-water torpedo reverberation. The model is based on Gaussian ray bundles in the form $|P(r, z)|^{2}=[\beta / \sqrt{2 \pi} \sigma(r) p, r] \exp \left(-0.5\left[\left(z-z_{r}\right) /\right.\right.$ $\left.\sigma(r)]^{2}\right)$, where $r$ is the horizontal range from the source to the field point, $z$ is the vertical depth of the field point, $z_{r}$ is the vertical depth along a central ray, $p_{r}$ is the horizontal slowness along a central ray, $\beta$ depends on the source, and $\sigma(r)$ is an effective standard deviation given by $\sigma(r)=(1 / 2) \max \left(\Delta z_{r}, 8 \lambda\right)$. Here, $\Delta z_{r}$ is the vertical distance between two adjacent rays and $\lambda$ is the wavelength at the field point. [Work supported by the Nayal Sea Systems Command (PMS-402 and PMS-406) and the Office of Naval Research (ONR-321).]

\section{0:45}

4aUW11. Shallow-water torpedo reverberation data modeling with the comprehensive acoustic system simulation. Ruth E. Keenan (Sci. Applications Intl. Corp., P.O. Box 658, Mashpee, MA 02649) and Henry Weinberg (Naval Undersea Warfare Ctr. Detachment, New London, CT 06320-5594)

The comprehensive acoustic system simulation (CASS) was used to track a 30-dB variability in the reverberation time series from 38 torpedo pings within a $4-\mathrm{km}^{2}$ area of the shallow-water U.S. Navy SOCAL range. This data set was extremely well modeled with a fairly simple bottom description. A thorough survey of the bottom characteristics in this area showed that the region transitioned from a current-swept rough-rock bottom to a mud-filled depression. The Gaussian ray bundles (GRAB) model, a component of CASS, simulated the acoustic propagation in this rangedependent environment. With the exception of the forward bottom loss for rock, the component scattering models proposed by the Applied Physics 
Laboratory, University of Washington [APL-UW 'TR 9407, AEAS 9501, October 1994] were used in the simulation. Twenty-nine interleaving torpedo pings traversed the rough-rock conditions. The model predictions were internally consistent with the data and the environment when a 6-dB value was assumed for the rough-rock forward bottom loss. [Work supported by the Naval Sea Systems Command (PMS-402 and PMS-406) and the Office of Naval Research (ONR-321).]

\section{1:00}

4aUW12. Nonadiabatic ocean acoustic environments and the flux density derivative. Timothy $\mathrm{H}$. Ruppel (Code 7173, Naval Res. Lab., Stennis Space Center, MS 39529-5004)

The accuracy of the adiabatic approximation is known to be suspect when applied to certain environments, particularly areas along the continental shelf. An issue in these environments is the degree to which the adiabatic assumption is valid. Transmission loss calculations using both parabolic equation and coupled mode models show that for steep slopes much more energy reaches the shelf from deep water than predicted by the adiabatic approximation. It has been shown that rapid variations in the energy flux as calculated from a parabolic equation model often indicate a breakdown of the adiabatic approximation and, therefore, significant mode coupling. This study connects these observations to the propagation physics and the standard coupled mode equations. The limits and strengths of using variations in the energy flux as an indicator of mode coupling are outlined. It is shown that this method of determining the degree of validity of the adiabatic assumption is valid in a large class of environments.

\section{1:15}

4aUW13. Optimum wideband detection of solid spherical objects of uncertain composition in the vicinity of a smooth sandy bottom. $M$. Wazenski, D. Alexandrou, and L. W. Nolte (Dept. of Elec. Eng., Duke Univ., Box 90291, Durham, NC 27708-0291)

Wideband detection of solid spherical elastic objects is approached via optimal detection and estimation theory in conjunction with acoustic scattering models for both the object and the seafloor. The objective is the time domain development of full field optimal decision methods for highfrequency active detection of a target in the vicinity of the seafloor. This parametric approach incorporates the inherent uncertainty surrounding the physical composition of both the object and seafloor. The acoustic model used for the spherical object is the well-known modal series solution and is parameterized by object size, density (object and medium), compressional wave speed (object and medium), and shear wave speed. This deterministic model is used to predict the target signature measured at an array of sensors. Acoustic modeling of the seafloor is performed through application of a modified point-scatterer model. This model provides a physical mechanism for describing the spectral scattering properties of the seafloor and is used to predict the spatial and temporal coherence of the scattered sound field. Illustrative detection examples are presented using both simulation and tank experimentation. Performance measurements are given by receiver operator characteristic (ROC) analysis. [Work supported by ONR: Ocean Acoustics.]

\section{1:30}

4aUW14. A seismic interface wave transducer. Eric Smith, Preston S. Wilson, John A. Behrens, and Thomas G. Muir (Appl. Res. Labs., Univ. of Texas, Austin, TX 78713-8029)

Use of seismic interface waves in applications such as buried object detection and echo ranging introduce special transduction needs. Because the spectrum of interface wave excitations can be very complicated, involving modes of different polarizations and speeds, a transducer is required that can select isolated modes as well as isolated frequencies. For applications in unconsolidated sediments, problems of consistency and stability must also be overcome. Experiments with such a transducer have been carried out on a natural hard-sand beach of the Gulf of Mexico. The device is an independently amplitude- and phase-controlled bidirectional oscillator coupled to the sediment. Preliminary results indicate that mode isolation is indeed possible, and also that high degrees of mechanical efficiency and consistency can be achieved in coupling to sediments. [Work supported by the Office of Naval Research.]

\section{1:45}

4aUW15. Automatic classification of low-frequency Arctic ambient noise. Michael V. Greening (Datavision Computing Services Ltd., 203-1545 Pandora Ave., Victoria, BC V8R 6R1, Canada), John M. Ozard, and Stanley E. Dosso (Esquimalt Defence Res. Detachment, FMO Victoria, BC VOS IBO, Canada)

A technique is described which can be used to automatically classify Arctic ambient noise data collected using an array of hydrophones suspended below the ice. The technique is currently designed for analyzing low-frequency $(5-60 \mathrm{~Hz})$ noise collected from the central Arctic pack ice. It is capable of distinguishing between nearby or distant active pressureridging events, thermal ice-cracking events, biological noise, cable strum, flow noise, and microseisms. Different noise types are distinguished by the power spectra, cross spectral matrices, noise directivity, and modal decomposition of the received sound. The technique is designed to be used in a real-time system which can automatically classify the dominant noise contained in the data over a specified time interval. This technique is applied to data obtained in the Lincoln Sea over 5 days in April 1988 to show how the ambient noise characteristics change with time during this period. Application of the algorithms produced a quantitative classification of the noise type that was clearly recognized in displays of the distinguishing measures. 


\title{
Meeting of Accredited Standards Committee S12 on Noise
}

to be held jointly with the

\section{U.S. Technical Advisory Group for ISO/TC 43/SC1 Noise and ISO/TC 94/SC12 Hearing Protection}

\author{
D. L. Johnson, Chair S12
}

EG\&G Special Projects, Albuquerque Operations, Albuquerque, New Mexico 87119-9024

P. D. Schomer, Chair, U.S. Technical Advisory Group (TAG) for ISO/TC 43/SC1, Noise (and Vice Chair S12) U.S. CERL, P.O. Box 4005, Champaign, Illinois 61820

H. E. von Gierke, Vice Chair, U.S. Technical Advisory Group (TAG) for ISO/TC 43/SC1, Noise 1325 Meadow Lane, Yellow Springs, Ohio 45387

E. H. Berger, Chair, U.S. Technical Advisory Group (TAG) for ISO/TC 94/SC12, Hearing Protection Cabot Safety Corporation, 7911 Zionsville Road, Indianapolis, Indiana 46268-1657

\begin{abstract}
Standards Committee S12 on Noise. Working group chairs will report on their progress for the production of noise standards. The interaction with ISO/TC $43 / \mathrm{SCl}$ and ISO/TC $94 / \mathrm{SC} 12$ activities will also be discussed, with reference to the international standards under preparation. the Chairs of the respective U.S. Technical Advisory Groups (H. E. von Gierke and E. H. Berger) will report on current activities of these intemational Technical Subcommittees under ISO.
\end{abstract}

Scope of S12: Standards, specifications and terminology in the field of acoustical noise pertaining to methods of measurement, evaluation and control; including biological safety, tolerance and comfort and physical acoustics as related to environmental and occupational noise.

\section{Session 4pMU}

\section{Musical Acoustics: Early Musical Instruments and General Topics}

\author{
Peter L. Hoekje, Chair \\ Physics Department 0150, University of Northern Iowa, Cedar Falls, Iowa 50614
}

Chair's Introduction-1:30

Invited Papers

$1: 35$

4pMU1. Early Flemish harpsichord string scalings and their acoustical implications. John Koster (The Shrine to Music Museum, Univ. of South Dakota, 414 E. Clark St., Vermillion, SD 57069)

Measurements of string lengths and diameters in historical harpsichords such as those made by the Ruckers family, active in Antwerp from 1579 to the 1680 s, are commonly used to calculate: (1) the pitch at which they were intended to be tuned, based on the assumption that strings were stressed to the practical limits of their tensile strength; and (2) the resulting tensions. Consideration of early music theory and workshop design practices have led to the proposal of altemative assumptions that some pre-Ruckers' makers employed shorter string lengths. With other variables remaining constant, higher calculated coefficients of inharmonicity result. The strings, however, were probably thinner, therefore resulting in inharmonicities similar to those of Ruckers' instruments. Further, coupling with sympathetically vibrating generally undamped strings of the earliest harpsichords might have reduced the theoretically 
calculated inharmonicities of the upper partials of bass and tenor strings. Other aspects to consider in light of early instrument makers' use of traditional geometrical methods of design include: (1) the Ruckers' slight lengthening of virginal (rectangular harpsichord) $\mathbf{E}$, F-sharp, and G-sharp strings intended to be tuned in meantone temperament, in which these pitches are flatter than in equal temperament; and (2) the development of reasonable plucking points.

2:05.

4pMU2. Influences of cornett lower end. P. L. Hoekje (Phys. Dept., Univ. of Northern Iowa. Cedar Falls, IA 50614-0150)

The lack of a distinct bell at the large end of the renaissance cornett is associated with some unique properties. The typical cornett has a conical shape with a length between 55 and $65 \mathrm{~cm}$, and an open end diameter of $2.5-3.5 \mathrm{~cm}$. Its range is from A3 to D6 (about $220-1175 \mathrm{~Hz}$ ). With all holes covered, it usually overblows a minor ninth from the first register to the second. Notes with open holes overblow an octave, and adjustment of tone-hole dimensions can bring the ratios of second- to first-mode frequencies close to 2:1 for diatonic scale notes. Besides benefitting tuning, this also improves low register response due to intermode cooperation. The cutoff frequency of the tone-hole lattice is typically between about 600 and $900 \mathrm{~Hz}$. Higher frequencies pass down the bore but are reflected back at the open end, whose cutoff frequency exceeds $3000 \mathrm{~Hz}$. This sustains several higher modes whose frequencies are strongly affected by the total bore length. The length directly affects the tuning of some third-register notes, and a second-register note can benefit when the bore length is adjusted so that a higher mode frequency lines up with a harmonic of the tone.

\section{Contributed Papers}

\section{2:35}

4pMU3. Mechanisms of vocal vibrato. Ingo R. Titze (Natl. Ctr. for Voice and Speech, Dept. of Speech Path. and Audiol., Univ. of Iowa, Iowa City, (A 52242)

A model of vocal vibrato is proposed that incorporates a late reflex (about $70 \mathrm{~ms}$ in loop delay) between the stretch and joint receptors of the vocal fold and the cricothyroid and thyroarytenoid muscle activations. The negative feedback loop is modeled with an adjustable gain and additional latencies in muscle contraction and rotation between the cricoid and thyroid cartilages. With these latencies and known mechanical properties of the laryngeal system, vibrato frequencies on the order of 5-7 $\mathrm{Hz}$ have been obtained.

\section{2:50}

4pMU4. Vibrational characteristics of pipe organ reed tongues. G. R. Plitnik and Ronald Knox (Dept. of Phys., Frostburg State Univ., Frostburg, MD 21545)

Pipe organ reed pipes sound when a fixed-free curved brass reed mounted on a shallot is forced to vibrate by an impressed static air pressure. The frequency dependence of these vibrating reed tongues was determined as a function of three variables. These were the thickness of the reed, the static air pressure within the reed boot, and the length of the reed allowed to vibrate. For each trial, two of these variables were kept constant while the third was altered. Experiments were conducted using three different types of shallots: The American (standard) style, the French style, and the German style. The results show that for each shallot, the frequency increases linearly with thickness and linearly with air pressure (over the normal operating range of the reed). For each of the shallots, frequency varies inversely with length when the other variables are held constant.

$$
\text { 3:05-3:15 Break }
$$

\section{3:15}

4pMU5. Computer modal analysis of percussion sounds: A preliminary study. James W. Beauchamp (School of Music and Dept. of Elec. Eng., Univ. of Illinois at Urbana-Champaign, Urbana, IL 61801)

Short time Fourier transform methods can be applied to the problem of modal analysis of percussion sounds, which are well known to contain inharmonic partials. One problem is that the Fourier transform is equivalent to a harmonically spaced filter bank, so it is not possible to position filter centers at arbitrary positions. Another problem is that, typically, percussion modes are closely spaced while their amplitudes change rapidly, which plays havoc with frequency versus time resolution limitations. For widely spaced modes, the first problem is solved by choosing the base analysis frequency to be an integral common divisor of the modal frequencies, so that each mode corresponds to a unique equivalent filter. When modes become too dense, they cannot be resolved, and bands of modes must be treated as indivisible entities in order to conserve the temporal behavior. For example, if a 20 -ms time resolution is desired, modes no closer than $50 \mathrm{~Hz}$ can be resolved. Results and ramifications of these limitations for analysis of percussion instruments such as tubular bells, timpani, and cymbals will be presented and discussed.

4pMU6. Computer simulation of drum sounds. James H. Irwin, Jr. (Elec. and Comput., Eng. and Technol. Dept., Bradley Univ., Peoria, IL 61625) and James $W$. Beauchamp (Univ. of Illinois at Urbana-Champaign, Urbana, IL 61801)

A difference equation derived from a physical model of a stiff membrane is used to simulate the acoustic signal produced by a drum head. The physical model was extended beyond the classical model by the inclusion of terms which represent viscous damping, air loading, and time-varying tension. Fixed phase vocoder and tracking spectral analysis show that the simulated sound contains correct theoretical frequencies when the damping and stiffness are set to zero. As stiffness is increased, the partial frequencies increase as predicted by plate theory. Simulations of zero (vacuum) and typical air loads show no significant changes. As a means of corroboration, measurements were made on an actual drum head mounted in a vacuum chamber. The drum signal was generated by striking the drumhead with a plastic tip mounted on a solenoid core and measured with an accelerometer mounted upon the drumhead. Spectral analysis results for this signal are qualitatively similar to the simulation measurements although they differ in some details. 
results were compared with the pitch model of Terhardt $e t$ al. [J. Acoust. Soc. Am. 71, 679-688 (1982)]. Both experiment and model show competition between spectral and virtual pitch and between cues at the nominal strike tone and the minor third. Generally the experiment finds the range of virtual pitch to be higher than predicted by the model. [Research supported by the Sligh Clock Company, Zeeland, Michigan, by the NIDCD, and by the NSF Research Participation for Undergraduates Program.]

\section{4:00}

4pMU8. Automatic recognition of musical notes. Jimmy H. Kapadia and John F. Hemdal (Dept. of Elec. Eng. and Comput. Sci., Univ. of Toledo, Toledo, OH 43606)

A system for the automatic recognition of musical notes was designed and implemented on a IBM-PC using commonly available hardware. The input to the system was a recorded piece of monophonic music while the output was its musical score. The recognition was carried out in the time as well as the frequency domains. Different characteristics of the note, like its frequency (or its name) and its time value, were found. The approach for this project was primarily from a digital signal processing (DSP) point of view. Various DSP fundamentals, like comb filter analysis and cepstrum analysis, were used for achieving the ends. The emphasis was more on trying out different approaches than on achieving a desirable result. Finally, an attempt was made to recognize a chord.
4pMU9. Signal processing for computer-assisted instruction of sight singing. Lloyd Smith and Rodger McNab (Dept. of Comput. Sci., Univ. of Waikato, Hamilton, New Zealand)

SST (Sight Singing Tutor) is a computer-assisted instructional program to teach the skill of singing melodies at sight. The program displays a melody on the screen, accepts acoustic input from a user, then evaluates the user's attempt to sing the melody. SST's primary signal processing tasks are frequency identification, note segmentation, and matching the singer's input against the test melody. SST tracks frequency using the well-known Gold-Rabiner algorithm [J. Acoust. Soc. Am. 46, 442-448 (1969)], averaging over 20 -ms frames in order to compensate for sampling error at higher frequencies. Notes are segmented using smoothing and grouping procedures based on frequency differences calculated in cents. The user's melody is matched against the test melody using a dynamic time warping (DTW) algorithm designed to match discrete melodic sequences [Mongeau and Sankoff, Comput. Humanit. 24, 161-175 (1990)]. SST displays the DTW match and presents a score, which takes into account both pitch and rhythm. SST works equally well with both male and female voices and allows the singer to sing the melody in his or her most comfortable octave; the prototype system has been shown to accurately identify and display notes ranging from $\mathrm{F} 2(87 \mathrm{~Hz})$ to $\mathrm{A} 5(880 \mathrm{~Hz})$.

THURSDAY AFTERNOON, 30 NOVEMBER 1995

ST. LOUIS H, 1:00 TO 3:15 P.M.

\title{
Session 4pNSa
}

\section{Noise: General Noise Control}

\author{
Carl D. Bohl, Chair \\ Central Institute for the Deaf, 818 South Euclid Avenue, St. Louis, Missouri 63110
}

\section{Contributed Papers}

\section{1:00}

4pNSal. An ad hoc method for control of generator noise. Ewart A. Wetherill (Paoletti Assoc., Inc., 40 Gold St., San Francisco, CA 94133)

A machine shop is housed in the same building as two large motor generators. Because of the need for overhead crane access to both facilities they are separated only by a partial-height wall. The resulting work environment is noisy and unpleasant although below the OSHA requirements for hearing protection. This paper describes two feasible methods of acoustical separation, one of which was aceptable to the owner but which has not yet been executed.

\section{1:15}

4pNSa2. The effect of duct cross-sectional area variation on acoustic and flow performance: Herschel Venturi tubes versus Universal Venturi tubes. A. Selamet (Dept. of Mech. Eng. and Appl. Mech., 120 W. E. Lay Automotive Lab., Univ. of Michigan, Ann Arbor, MI 48109-2121)

Following an earlier work by Selamet et al. [Proc. Inter-Noise 95, 425-430 (1995)], the present analytical, computational, and experimental study investigates the effect of duct cross-sectional area variation on the wave attenuation and flow performance. Two different Venturi configurations, Herschel Venturi tubes and Universal Venturi tubes, are compared. Four Venturi tubes are fabricated in each category witl duct to throat cross-sectional area ratios varying from 2 to 16 and used in the experi- mental effort. The results from these configurations are then discussed in terms of the acoustic attenuation versus flow efficiency.

\section{1:30}

4pNSa3. Coupled response and noise of roller chain drive systems. S. P. Liu, ${ }^{\text {a) }}$ S. I. Hayek, K. W. Wang, M. W. Trethewey (Penn State Univ., University Park, PA 16801), and F. H. K. Chen (GM Res. \& Development Ctr., Warren, MI 48090)

A chain drive system consists of a closed loop roller chain wrapped around two or more sprockets. One of the most significant noise sources in an operating roller chain drive emanates from the repeated impacts between the chain links and sprocket teeth during their meshing. Previous studies on the local-global meshing of the chain with the sprockets only considered the dynamic transverse motion of an axially moving chain while uncoupled from the sprockets. In this study, the analysis is extended to axial-transverse motions of the moving roller chain coupled with the dynamic response of the rigid sprockets over which the chain is wrapped around. The analysis thus integrates the local impact meashing to the global response of the chain-sprocket systern. Numerical simulations of the analytical model showed that the coupling effects between the two sprockets, the two chain spans, and the chain-sprocket meshing impulse intensity increase with decreasing sprocket inertia and chain longitudinal stiffness Controlled tests on a roller chain/two-sprocket system verified the results of the numerical simulations; that the meshing noise SPL is directly related to the chain speed and its vibrational characteristics. [Work supported by the GM Corp. ${ }^{\text {a) }}$ Currently at GM Research \& Development Center. 
analysis of the interrelation between emergency situations at thermal power plants of Russia and Ukraine and the noise load on the workers (1979-1989), while artesting working places at the enterprises of building materials in Kiev (1992-1994).

\section{$\mathbf{2 : 3 0}$}

A preliminary model experiment showed that the insertion loss for a random edge barrier exceeded that of a straight edge barrier of the same average height [J. Acoust. Soc. Am. 97, 3392(A) (1995)]. Based on this promising result, a three-level full factorial model experiment has been conducted on random edge barriers. The top of the barrier is a strip of sheet metal of fixed average height but made randomly triangular by straight line culs. A random number generator was used to specify the height variations $\Delta y$ (scaled to a prescribed maximum value) at fixed intervals $\Delta x$ along the barrier. Nine different random edges provide a range of 9-37 $\mathrm{mm}$ in $\Delta x$ and 5-18 mm in $\Delta y$. An electric spark was the sound source, a 1/8-in. $\mathrm{B} \& \mathrm{~K}$ condenser microphone the receiver. The frequency range of the measurements is $5-2 \mathrm{l} \mathrm{kHz}$, the Fresnel number range 2-20. ln most of the measurements the random edge barriers showed 3-8 dB more insertion loss than the straight barrier. For several cases, however, the straight barrier was better. [Work supported by TxDOT.]

\section{2:00}

4pNSa5. Acoustical testing and treatment of elementary schools, St. Louis International Airport environs. Jerry W. Schweiker, Philip J. Wentz, and Robert Taylor, Jr. (Eng. Dynam. Intl., 8420 Delmar Blvd., Ste. 303, St. Louis, MO 63124-2186)

Since 1986 a school acoustical treatment has been funded by Airport Community Programs Office (ACPO), St. Louis International Airport. The program has included selection of candidate schools, inside-outside noise tests, providing acoustical trealment recommendations, construction, and the postconstruction testing of elementary schools within the environs of St. Louis Intemational Airport (Lambert Field). This paper summarizes the results of the first eight schools. Three schools remain in the program and await funding for the construction phase. Although the instrumentation and the type of noise tests has varied somewhat over the years, the primary goal was to reduce the classroom noise to below the $L_{\mathrm{eq}} 45$ level per FAA guidelines. SEL and 1/3-oct band data from overflights were also evaluated in some schools. The improvement in classroom $L_{\mathrm{eq}}$ varied from 8 to 15.7 dBA in the classrooms tested. The acoustical improvements specified included acoustic windows and doors, interior acoustical treatment, new roofing, and air conditioning. The cost of construction varied from $\$ 253000$ to $\$ 500000$ per school.

\section{2:15}

4pNSa6. Methods of group evaluation of noise load in industry. Alexander A. Menshov and Mark V. Levin (Dept. of Physical Factors, Inst. for Oecupational Health, 75 Saksagansky St., Kiev, Ukraine 252033)

Methods of evaluation and regulation of individual noise load for the analysis of its inlerrelation with quality, labor productivity, and emergency situations at production areas have been elaborated in detail. It is much more difficult to evaluate noise load for the whole group of workers even within the boundaries of one technological zone. For the production area attestation, from the viewpoint of the noise factor, it was proposed that the noise load of a group of workers should he evaluated as a percent ratio of the number of persons whose noise load is higher than the standard, to the total number of workers in the evaluated group. This index can be called the "coefficient of noise attestation" (CNA). For the analysis of the interrelation between quality, labor productivity, emergency situations at the production areas, and the noise load, it is proposed that the latter be evaluated as a quotient from the division of the sum of the noise load excesses for each of the workers by the number of workers in the evaluated group. This second index can be called the "coefficient of noise load on workers" (CNLW). These coefficients are used in new standards of Ukraine (N 2325 "Noise. Terms and definitions," 1993), in a draft standard of Ukraine ("Noise. Methods of definition of noise load of workers," 1995), and in the
4pNSa7. The meaningfulness of reductions in aircraft noise exposure Pearsons (BBN Systems and Technologies, 21120 Vanowen St, Canoga Park, CA 91303)

As quieter aircraft have begun to replace some of the noiser ones in the civil air transport fleet, aircraft noise exposure levels in certain airport neighborhoods have declined to some degree. Federal agencies with interests in aviation noise have not yet offered policy guidance for gauging the smallest decrease in aircraft noise that may be considered meaningful under these circumstances in airport neighborhoods. An empirical study of this issue was thus underken in residential areas near a large airport at which noise exposure levels had declined by several decibels over the last few years. Very few neighborhood residents either noticed a decrease in aircraft noise in the recent past or decribed themselves as less annoyed by aircraft noise than in prior years.

\section{2:45}

4pNSa8. Blast sound propagation over hilly land. Takehiro Isei, Hiroyuki Imaizumi, Sunao Kunimatsu, Michiaki Kinoshita (Safety Eng. Dept. Natl. Inst. for Resources and Environment, 16-3, Onogawa, Tsukuba 305, Japan)

A blast sound propagates over a long range, because of its large sound power of sources and low-frequency components. Blasting operations are often conducted at a hilly side and as a result, sometimes the sound of the blast influences the life environment beyond the hilly undulations. At both hilly sites with heights of up to several tens of meters and flat lands, excess attenuation of blast sound was measured at points of several hundred meters away. Frequency spectrums of excess attenuation beyond the hilly side show that (1) up to around $100 \mathrm{~Hz}$, excess attenuation increases in proportion with frequency, and for more than $100 \mathrm{~Hz}$, it shows mostly constant values of around $40 \mathrm{~dB}$; (2) shapes of frequency spectrum of the excess attenuation show similar pattern with the ones of flat land; and (3) shielding effects of hills mainly appear at the frequency range around $100-250 \mathrm{~Hz}$. From these results, the authors discuss the possibility of sound propagation modeling by extending usual models for flat land.

\section{3:00}

4pNSa9. Sound energy distribution at an intersection of underground tunnel: Small scale experiment. Hiroyuki Imaizumi and Takehiro Isei (Safety Eng. Dept., Natl. Inst. for Resources and Environment, 16-3 Onogawa, Tsukuba 305, Japan)

Underground spaces generally have complex networks of tunnels which include many intersections. When a disaster such as a fire and an earthquake breaks out there, correct transmission of sound information has an important role in order for people to evacuate from underground safely [Fujimiya and Miura, J. INCE Jpn. 6(5) (1982)]. From the above point of view, sound energy distribution characteristics at an intersection of a scale model tunnel were measured under several conditions. The scale model tunnel had a square cross section, and experiments were carried out under acoustically hard and soft conditions. Impulsive sound from an electrical sound source indicated high reproductivity and omnidirectional characteristics from a preliminary experiment in the anechoic room. In a case where the scale model tunnel has an orthogonal branch, it was observed that low-frequency components were mainly propagated toward the branch, and this tendency was changed according to the angle of intersection. Furthermore, qualitative numerical simulation on sound propagation in a tunnel with an intersection was developed and the results agreed well with experimental results. in airport neighborhoods. Sanford Fidell, Laura Silvati, and Karl 


\title{
Session 4pNSb
}

\author{
Noise: Active Noise Control \\ Jason T. Weissenburger, Chair \\ Engineering Dynamics International, 8420 Delmar Boulevard, \#303, St. Louis, Missouri 63124
}

\section{Contributed Papers}

3:30

4pNSb1. Quiet unlined HVAC ductwork: Using active silencing to obtain NC-35 in buildings without fibrous materials. Steve Wise, Lawrence J. Gelin, Kirk G. Burlage, and Susan H. Dineen (DIGISONIX, 8401 Murphy Dr., Middleton, WI 53562)

Fan noise in HVAC ducts has traditionally been attenuated with fibrous internal duct liner or with passive silencers constructed with porous fill material. Now, with active noise control, it is possible to cancel the loudest frequencies in the fan noise spectrum using inert components on the ductmicrophones and loudspeakers. Proper selection of fan type, optimal duct design, and appropriate active noise control system configuration can achieve acceptable noise levels in all octave bands in the sensitive building spaces. Many recent examples of actual installations are given, including systems on air handlers up to $25000 \mathrm{cfm}$ capacity. Systems are applied, not only the central station air handlers, but also to secondary fans such as those used in fan-powered VAV boxes. Application considerations such as duct design and energy efficiency will be noted in this paper along with the specific room acoustical requirements and system performance.

\section{3:45}

4pNSb2. Active sound extraction for noise control in the presence of duct mean flow. Sameer I. Madanshetty (Aerospace and Mech. Eng., Boston Univ., 110 Cummington St., Boston, MA 02215) and Boa-Teh Chu (Yale Univ., New Haven, CT 06520)

A method of controlling noise level in duct flows is described. The method is based on the principle of energy extraction by active source(s), rather than by wave cancellation as in "antisound." As such, the method of energy extraction is robust; it does not need the delicate signal processing, perfect phase, and amplitude matching, crucial to sound cancellation. An improved control strategy useful for both low and high frequencies is described, and its effectiveness in the presence of mean flow is analyzed. It is found that in the presence of through flow the control is effective in extracting energy from any waves traversing it. As the duct flow Mach number increases the control appears to be less effctive for waves produced upstream of it but it is more effective for the waves produced downstream of the device. Further, this conclusion remains valid whether the detector is placed on the upstream or downstream side of the control device.

\section{4:00}

4pNSb3. Primary and secondary sound transfer functions in an active noise control earplug. Keith T. Olree and Thomas R. Harley (Natl. Cir. for Phys. Acoust., Coliseum Dr., University, MS 38677)

Active noise control in a headset or earplug typically attempts to lower the sound level at an error microphone located close to the ear. If the transmission path between the error microphone and the eardrum for the primary noise is different to the path for the secondary noise, a reduction at the error microphone may not lead to a reduction at the eardrum. In fact, if these paths are sufficiently different a reduction at the error microphone may actually lead to amplification at the eardrum. An example of a possible cause for differing transmission paths is the bone conduction effect that becomes noticeable in occluded ear canals. In this study, a probe microphone was inserted into the ear canal of human subjects. This microphone was used to measure the pressure near the eardrum for primary and secondary sound sources. The pressure was also measured at an error microphone of an active noise control earplug. These measurements will be presented and some of the implications for active noise control will be given.

\section{$4: 15$}

4pNSb4. Experimental results using active control of energy density. Scott D. Sommerfeldt (Dept. of Phys. and Astron., Brigham Young Univ., Provo, UT 84602), John W. Parkins, Young C. Park, and Zane M. Rhea (Penn State Univ., University Park, PA 16802)

Previous research reported has presented numerical results obtained, indicating that one can often achieve improved global control of an enclosed acoustic field by minimizing the acoustic energy density, rather than the acoustic pressure. Inexpensive sensor probes have been developed that are capable of sensing the pressure and velocity components of the acoustic field, for incorporation into an adaptive control system that minimizes the energy density. The control system has been implemented within an enclosed sound field and has been used to compare the global attenuation achieved by minimizing either the squared pressure or the energy density. The control system is capable of implementing control with multiple sources and/or sensors. Results are shown for the low modal density case in the enclosure and indicate the improved global attenuation that can be achieved using energy density control. As well, the dependence of the controlled field on error sensor location is shown to be substantially weaker when controlling energy density than when controlling squared pressure. [Work supported by NASA Langley Research Center.]

\section{$4: 30$}

4pNSb5. Local algorithms for controlling an active piston damping system. Vladimir V. Arabadzhi (Inst. of Appl. Phys., Russian Acad. of Sci., Dept. of Hydrophys. and Hydroacoust., 603600 Nizhny Novgorod, Russia)

A scattering and/or emitting surface with local (of small wave dimensions) piston emitters located directly on it is considered. The problem of active sound damping by the quantity of prior information on the wave field and surface properties are classified. It is shown that in some practically significant cases one may do without traditional accumulation in a damping system of the entire information "imprint" of the wave field. The aim of the paper is the construction of the most local algorithms. Four control algorithms are described in the paper: (a) the algorithm of the maximum of the total average for the period of the absorbed power on the pistons, (b) the algorithm of the iterative compensating of the sound pressure on pistons, (c) the algorithm of the compensating of the volume velocity, (d) the algorithm of the physical surface in accordance with the incident wave. The results of the action of algorithms (a)-(d) is the trans- 
formation of the distribution of normal oscillating velocities on a given surface to the region of high (weakly emitting) space frequencies. A joint solution of the problems of radiating and scattering damping is achieved too.

\section{$4: 45$}

4pNSb6. Parametric coating. Vladimir V. Arabadzhi (Inst. of Appl. Phys., Russian Acad. of Sci., Dept. of Hydrophys. and Hydroacoust., 603600 Nizhny Novgorod, Russia)

A control algorithm for the multilayer parametric coating is suggested. The frequency of layer parameter manipulations is much larger than the incident wave frequency and the total multilayer coating thickness is much less than the incident wavelength. This denotes the space-time locality of the damping system. The coating provides the boundary conditions of the acoustical blackbody at low frequencies for a surface of arbitrary form and wave dimensions. This case includes the incident waves absorption and the forward scattering without backward scattering. The algorithm does not require wave field measurements. It assigns the law of coating parameters to time changes only (instantaneously on the whole surface). Algorithm realization may be based on the electrorheological liquids which make fast transformations from the liquid state to the solid one and back due to the applied electric voltage at all usual temperatures. The results of numerical simulation are presented.

\section{5:00}

4pNSb7. Parametric silencer. Vladimir V. Arabadzhi (Inst. of Appl. Phys., Russian Acad. of Sci., Dept. of Hydrophys. and Hydroacoust., 603600 Nizhny Novgorod, Russia)

Total dimensions of the silencer of exhaust gas noise usually are in finite relations with the sound wavelength, corresponding to the engine assigned frequency. A silencer design independent of assigned frequency is suggested. An ultrasound sirenlike flux cyclical switch ensures the
$\mathrm{N}$-cascade up-conversion of the pulsing frequency into the ultrasound frequency range, where the small-dimensional sound absorbers may be used. $N$ cascades ensure low-frequency pulsing amplitude decreasing by $2^{N}$ times. The output cascade tube diameter must be more than the input tube diameter by $\sqrt{2}$ times to save the average in time total flux cross section. This circumstance is the main factor that limits a decrease in the size of the silencer dimensions. The results of experimental investigations of the onecascade system are presented.

\section{$5: 15$}

4pNSb8. The local algorithms of autoresonant absorption of the energy of bending waves in elastic plates. Vladimir V. Arabadzhi (Inst. of Appl. Phys., Russian Acad. of Sci., 603600 Nizhny Novgorod, Russia)

For the simplest model一an infinite elastic plate-three control algorithms (local in space and time) are studied by a normal point shift to achieve the maximum of absorbed power. They are: (a) the algorithm of half-retum, (b) the algorithm of the random search for the maximum of the instantaneous absorbed power, (c) the continuous algorithm of the resonant absorption. These algorithms provide the exit to the trajectory of the resonant absorption during the time much less than the minimum time scale of the damped wave, i.e., knowing either its period, or its length. The considered algorithms differ in the necessary volume of the a priori information on the plate parameters and in the power of the "technological" high-frequency radiation caused by rapid manipulations with the boundary condition. The speed of response of sensors and final control elements is supposed to allow the formation of arbitrary kinematic characteristics of the boundary. The conditions for the effective operation of the algorithms in the case of plates with finite dimensions are formulated. The algorithms may be used to decrease the quality of mechanical systems made up of elastic plates.

\title{
Session 4pPA
}

\section{Physical Acoustics: Thermoacoustics}

\author{
W. Patrick Arnott, Chair \\ Desert Research Institute, Atmospheric Sciences Center, University of Nevada, P.O. Box 60220, Reno, Nevada 89506-0220
}

\section{Contributed Papers}

\section{$1: 30$}

4pPA1. Experimental results from four thermoacoustic cryocoolers. Thomas J. Hofler and Jay A. Adeff (Dept. of Phys., Naval Postgrad. School, Monterey, CA 93943)

The experimental results from a thermoacoustic cryocooler project will be reported. Four different versions of the cryocooler were constructed and the coldest temperature achieved was $180 \mathrm{~K}$. While this temperature result fell short of our goals, a number of interesting discoveries were made. One engine performed very much worse than the linear theory predicted, the pitfalls associated with compact and efficient acoustic resonators were discovered, and lower SWR standing waves were found to enhance engine performance. [Work supported by NRL and ONR.]
$1: 45$

4pPA2. A model of the temperature discontinuities found between the elements of thermoacoustic engines. James $R$. Brewster and Richard Raspet (Dept. of Phys. and Astron., Univ. of Mississippi, University, MS 38677)

In experimental investigations of thermoacoustic engines, the temperature difference required to drive these devices at high amplitude has been found to be markedly higher than that predicted by linear theory. This discrepancy is due in part to a significant difference in temperature that has been observed between stack sections and their adjoining heat exchangers. It will be shown that the existence of a step in the longitudinal temperature profile is a finite-amplitude effect, not encompassed by the linear theory, and is essential if heat is to be transferred between elements of the engine. 
Physical insights obtained from a simple model of the heat transfer process based on the assumption that the working fluid is inviscid and incompressible will be presented. This will be followed by consideration of how the existence of viscosity and fluctuations in the density of the working fluid affect this model. [Work supported by the Office of Naval Research.]

\section{2:00}

4pPA3. A simplified model of a thermoacoustic refrigerator. Andrea Prosperetti and He Yuan (Dept. of Mech. Eng., Johns Hopkins Univ., Baltimore, MD 21218)

The simplified approach to thermoacoustic prime movers described at earlier ASA meetings is extended to the case of heat pumps. The mathematical model is described and a weakly nonlinear theory is developed. The impact of the gas pressure, stack geometry, resonator shape, and other design parameters is explored. [Work supported by the Office of Naval Research.]

\section{2:15}

4pPA4. Comparative study of heat exchangers for thermoacoustic devices. George Mozurkewich and Steven R. Murrell (Ford Motor Co. Res. Lab., Maildrop 3028 SRL, P. O. Box 2053, Dearborn, MI 48121-2053)

Among the critical elements of a thermoacoustic device are its internal heat exchangers. This work quantifies the behavior of actual heat exchangers in situ in an operating thermoacoustic refrigerator. Each heat exchanger consisted of a specified arrangement of metal in contact both with oscillating gas adjacent to a thermoacoustic stack and also with water bearing tubes. Measured quantities were the temperature $T_{s}$ of the adjacent end of the stack, the temperature $T_{w}$ of the water flowing through the tubes, the change of water temperature $\delta T$ between tube inlet and outlet, and the water flow rate. The heat-transfer rate $Q$ was determined from the latter two quantities. The overall heat-transfer conductance $G$, defined by $G=Q /\left(T_{s}-T_{w}\right)$, is determined by three quantities: the gas-to-metal temperature drop, the fin efficiency, and the metal-to-water temperature drop. Raw experimental results will be presented for several gas-side heatexchanger geometries, including copper screen, reticulated aluminum foam, and bundled copper tubes. Where possible, comparisons will be made to simple theoretical expectations.

\section{$2: 30$}

4pPA5. Resonator coiling in thermoacoustic engines. Jeffrey $R$. Olson and Gregory W. Swift (Condensed Matter and Thermal Phys. Group, Los Alamos Natl. Lab, Los Alamos, NM 87545)

Coiling the resonator of a thermoacoustic engine is one way to try to minimize the engine's size. However, flow in bent pipes is known to alter the fluid flow pattem because of centrifugal forces. Theory and measurements will be presented on the energy dissipation caused by oscillating flow in curved pipes. Measurements have been taken using free oscillations of liquids in $U$-tubes, and using a thermoacoustic engine with straight and bent resonators. [Work supported by the TTI program of the US Department of Energy, and by the Tektronix Corporation.]

\section{2:45}

4pPA6. Turbulent losses in thermoacoustic resonators. D. $L$ Gardner, J. R. Olson, and G. W. Swift (MS K764, Los Alamos Natl. Lab, Los Alamos, NM 87545)

Minimizing losses resulting from turbulent flow in thermoacoustic resonators while preserving compactness is of concern. Iguchi et al. [Bull. JSME 25, 1398 (1982)], using an extension of the Blasius turbulent friction factor, determined a formulation by which turbulent losses in pipes with oscillating flows could be predicted. This formulation is applied in a preliminary effort to predict acoustic power dissipation. These predictions are compared with acoustic power flow measurements made using a thermo- acoustic engine having a straight pipe. Additional losses are expected if the resonator is not straight. Measurements using an engine having corkscrew or mitre-jointed resonators are compared to the work of Olson et al. (manuscript in progress). [Work supported by DOE.]

$$
\text { 3:00-3:15 Break }
$$

\section{$3: 15$}

4pPA7. Thermodynamic properties of helium-xenon mixtures for thermoacoustic refrigerators. John J. Hurly, Steven J. Boyes, and James W. Schmidt (Natl. Inst. of Standards and Technol., Thermophys. Div., Gaithersburg, MD 20899)

The equation of state and the speed of sound have been measured for helium, xenon, and three helium-xenon mixtures in the temperature and pressure ranges of interest for designing thermoacoustic refrigerators. The results have been correlated with a virial equation of state and can be used to optimize the refrigerator designs. [Work supported by the Office of Naval Research.]

\section{$3: 30$}

4pPA8. Shipboard electronics thermoacoustic cooler. D. McKelvey, S. Ballaster (Naval Postgrad. School, Monterey, CA 93943), and S. Garrett (Penn State Univ., State College, PA 16804)

A thermoacoustic refrigerator that was optimized for preservation of biological samples in space, was modified for use as a cooler for the CV-2095 shipboard radar electronics rack. The thermoacoustic cooler was tested in the laboratory and demonstrated at sea aboard USS DEYO (DD989). In the laboratory, using a calibrated heat load, the data acquisition system was able to account for the total energy balance to within $4 \%$. At the highest operating power aboard ship, $227 \mathrm{~W}$ of acoustic power was used to provide $419 \mathrm{~W}$ of useful cooling power, corresponding to a coefficient of performance of 1.85 . Taking into account the $54 \%$ electroacoustic efficiency of the loudspeakers, the shipboard electronics thermoacoustic cooler (SETAC) provided $1 \mathrm{~W}$ of cooling for each watt of electrical power input. [Work supported the Office of Naval Research and the Navy Science Assistance Program.]

\section{$3: 45$}

4pPA9. Radial wave refrigerator driven by a plane-wave prime mover. W. Patrick Arnott (Desert Res. Inst., Univ. Nevada, P.O. Box 60220, Reno, NV 89506) and Richard Raspet (Univ. of Mississippi, University, MS 38677)

Previous heat-driven thermoacoustic refrigerators have used a design that compromises the performance of both the prime mover sound source and the refrigerator. The prime mover and refrigerator were placed next to each other in the resonator, and neither could be placed at the optimal performance location; otherwise, the efficiency or output of the one placed well away from the optimal location would be low. A radial wave refrigerator in a wide cylindrical resonator driven by a plane-wave prime mover from a narrow resonator allows for a relatively compact design, and more importantly, for optimization of the performance of both elements. Results of numerical analysis for the radial wave refrigerator driven by a planewave prime mover will be presented. [Work supported by the Office of Naval Research.]

\section{$4: 00$}

4pPA10. Optimization of thermoacoustic engine design variables for maximum performance. Brian L. Minner, Luc Mongeau, and James E. Braun (1077 Ray W. Herrick Labs., Purdue Univ., West Lafayette, IN 47907-1077)

Thermoacoustic engine prototype design typically proceeds based on an intuitive, trial and error approach. This study investigates the benefits of performing a systematic optimization of design parameters in a thermoacoustic engine for given operational requirements. The design optimiza- 
tion seeks to minimize a cost function of performance using the simplex method. This method allows constraints to be readily implemented without need for gradient evaluations. Parameter space maps are generated for visualization of solution sensitivity. System analysis is performed with the assistance of DeltaE, a commercially available software tool which solves the 1-D lossy wave equation in resonator sections and Rott energy and wave equations in stack sections. The solution is therefore subject to the low-amplitude (linear) flow assumptions and conduction mode heat transfer assumptions in heat exchangers. The optimization and space mapping tools systematically write input decks, execute DeltaE, and evaluate results. Optimization of up to 13 geometric, fluid, and material properties has been explored, producing encouraging performance predictions. Application of this strategy to design modifications of documented prototypes and resulting benefits of systematic optimization will be illustrated. It is anticipated that the optimization scheme will be useful in enhancing performance of future thermoacoustic engine prototypes

\section{$4: 15$}

4pPA11. Numerical study of the effective impedance of an idealized thermoacoustic stack. A. S. Worlikar (Dept. of Mech. Eng., Johns Hopkins Univ., Baltimore, MD 21218), R. Klein (Institut für Technische Mechanik, 52056 Aachen, Germany), and O. M. Knio (Johns Hopkins Univ., Baltimore, MD 21218)

A low-Mach-number, compressible flow, simulation model is used to compute unsteady oscillatory flow in the neighborhood of a thermoacoustic stack. The model relies on a vorticity-based formulation of the mass, momentum, and energy conservation equations. The numerical scheme incorporates a highly efficient construction which combines a domain decomposition boundary Green's function formulation with fast Fourier inversion methods. Computed results are used to calculate the effective im- pedance of the device, which accounts for contributions of both linearized acoustic phenomena and nonlinear inertial effects. The dependence of this effective impedance on stack configuration and on acoustic driving amplitudes and frequencies is also analyzed. [Work supported by the Office of Naval Research.]

\section{4:30}

4pPA12. Holographic interferometry: An approach to study the unsteady temperature fields in the stack region and its neighborhood. C. Herman, M. Wetzel, and J. Wagner (Dept. of Mech. Eng., Johns Hopkins Univ., Baltimore, MD 21218-2686)

Holographic interferometry combined with high-speed cinematography is a measurement technique, which allows the investigation of unsteady temperature distributions without affecting the physical process. Therefore it is the most suitable measurement technique for the investigation of the oscillating temperature field in the stack region and its neighborhood. In order to apply holographic interferometry, vibrations of the experimental setup have to be kept below a fraction of the wavelength of the used laser light, $514 \mathrm{~nm}$. The first research efforts were focused in the design and verification of a feasible experimental setup which satisfies the requirement of low vibrations. Currently, temperature measurements, applying holographic interferometry to our thermoacoustic refrigerator model, are carried out. From the obtained interferometric fringe patterns the tempera ture distribution can be reconstructed quantitatively applying digital image processing. Results of these measurements will yield valuable information about the heat transfer mechanism occurring in the stack region and its neighborhood, which can be used to improve the heat exchanger design. [Work supported by the Office of Naval Research; Martin Wetzel is also supported by a scholarship from DAAD.]

\title{
Session 4pSA
}

\section{Structural Acoustics and Vibration: Statistical Methods in Complex Structures II}

\author{
Victor W. Sparrow, Chair \\ Graduate Program in Acoustics, Pennsylvania State University, 157 Hammond Building, State College, Pennsylvania 16804
}

Invited Papers

$1: 30$

4pSA1. Statistical phase analysis-The phase behavior of complex structures. Richard H. Lyon (RH Lyon Corp., Cambridge, MA 02138)

The magnitude and phase of a transfer function are often thought of as complementary or conjugate variables, and there may be special relationships between them. Statistical energy analysis (SEA) is a procedure for estimating the magnitude of a transfer function, not only of a single, directly excited structure, but of several structures joined together. In some situations, as in problems of machinery diagnostics and signal transmission in drill piping, the phase of the transfer function is important in determining system performance. If the structure is complex, a statistical estimate of the phase may be the best one can hope for, and may also be adequate. For this reason, an investigation has been done on the statistical properties of the phase of complex structures, first of directly excited structures, and, recenty, of joined structures to see if some of the relatively simple results of SEA are reflected in statistical phase analysis (SPA). This paper will review the approach taken to understanding the phase in complex structures and some of the results thus far.

\section{2:00}

4pSA2. Diffuse energy propagation on heterogeneous plates: Structural acoustics radiative transfer. Joseph A. Turner and Richard L. Weaver (Dept. Theoret. and Appl. Mech., Univ. of Illinois, Urbana, IL 61801)

The propagation of diffuse energy on an unwetted heterogeneous plate is examined using a diagrammatic approach. The heterogeneities are assumed to lightly couple the membrane and flexural waves. The energy propagation is formulated in terms of the Bethe-Salpeter equation. This equation is further reduced to a radiative transfer equation in limit that the attenuations per wave number are small, i.e., when the heterogeneities are weak. This approach allows the diffuse energy propagation to be calculated as a function of space, time, and propagation direction. Solutions of the derived radiative transfer equation are presented for the simple case of 
attached heterogeneities in the form of delta-correlated springs excited by an extensional point source launched in a single direction. The results show the evolution and mode conversion of the extensional, shear, and flexural energy densities across the plate as a function of time. A similar approach is expected to apply to the more complicated case of submerged complex structures. [Work supported by ONR.]

2:30

\section{4pSA3. Scattering from complex elastic structures. Douglas M. Photiadis (Naval Res. Lab., Washington. DC 20375-5000)}

The scattering cross section of elastic structures is modified in a nontrivial fashion by the presence of effectively random internal structure. The locus of the highlights in frequency-angle space can often be modeled reasonably by considering the behavior of the associated average structure, but the influence of the internal structure on the levels of the scattering cross section cannot be determined in this way. In terms of the development of effective scattering models, the mean Green's function does not contain enough information in this context and higher-order averages are required, at a minimum the mean intensity $\left(G G^{*}\right)$. The perturbative computation of such quantities is relatively well understood for a medium which is uniform, but for the structures of most interest, those whose properties on average vary spatially, the problem is much more difficult. The theoretical approach to the solution in this case will be discussed, and the approximate results obtained will be compared with the results from direct Monte Carlo simulations.

\section{3:00}

4pSA4. Waves in random media: Coherent and fluctuating parts, energy distribution. Samuil A. Rybak (N. N. Andreyev Acoustics Inst., 117036 Shvemik Str. 4, Moscow, Russia)

Waves in media with fluctuating parameters are described with the help of a Green's function method. For the average field, the integral Dyson equation is formulated and its solution is obtained by means of the Bourret approximation. The average field (coherent part) decays exponentially with distance from the point source while the fluctuations grow. The Bethe-Salpeter equations are formulated for the average energy distribution. The "ladder" approximation then gives a system of transport integral equations which resemble heat transport equations. For the validity of the theory some threshold level of dissipation in the medium is necessary. The distribution of the fluctuations of the parameters is taken to be Gaussian. The solutions for energy distribution have an exponential decay with the index proportional to the root of the dissipation coefficient of the medium. In waveguides with fluctuating parameters the wave modes are coupled and both the coherent parts of these modes and the energy distributions are not independent. The energy fluxes become equal for traveling normal waves. Elastic plates with fluctuating parameters are examples of such waveguides for coupled longitudinal and flexural waves. Verification of the theory was made with the help of direct calculations for some simple models.

\section{3:30-3:45 Break}

\section{Contributed Papers}

3:45

4pSA5. Ultrasonic band gaps in aggregates of sintered aluminum beads. Joseph A. Turner and Richard L. Weaver (Dept. Theoret. and Appl. Mech., Univ. of Illinois, Urbana, IL 61801)

An experimental examination of the transmission power spectrum of a block of sintered aluminum beads is discussed. Periodic structures such as these have complicated dispersion curves that contain stop bands and pass bands. Thus the power transmission spectrum for the agglomeration of sintered beads is strikingly different than that for a solid aluminum block. The positions of the observed band gaps are shown to agree with theoretical predictions. The width of the bands is directly related to the sintering level and the corresponding coupling between beads. Unlike previous onedimensional work, the medium considered here is three dimensional. Also, the rare modes that may lie in the band gaps or those near the edges of the bands are expected to localize. Therefore, this sintered bead structure is a promising candidate for the discovery of localizing ultrasound. [Work supported by NSF.]

\section{4:00}

4pSA6. Diffuse wave energy transport in multicoupled, one-dimensional Anderson localizing systems. R. L. Weaver (Dept. of Theoret. and Appl. Mech., Univ. of Illinois, Urbana, IL 61801) and John Burkhardt (Indiana Univ.-Purdue Univ., Ft. Wayne, IN 46805-1499)

The spatial and time domain evolution of energy density in a multicoupled, one-dimensional disordered system is investigated. Scaling theory predictions are presented for both localization lengths and rates of diffuse transport. Scaling arguments suggest that localization lengths equal $\pi \rho D_{0}$, where $\rho$ is the modal density per unit length and $D_{0}$ is the bare diffusivity. Additionally, the rate of diffuse energy transport over a distance $L$ is found to scale as $\rho L$. These predictions are compared with the behavior of a numerical model for an Anderson localizing system. The system modeled is a cylindrical membrane disordered by the introduction of a random foundation of springs. [Work supported by ONR.]

\section{4:15}

4pSA7. Spectral statistics for systems with ray splitting. Robert $\mathrm{N}$. Oerter (SFA, Inc., c/o Naval Res. Lab., Code 7131, Washington, DC 20375-5350), Ed Ott, Thomas M. Antonsen, Jr., and Paul So (Univ. of Maryland, College Park, MD 20742)

A finite reverberant system having a discontinuity in some physical parameter will exhibit the splitting of ray trajectories in the high-frequency limit. It is known that this ray splitting can increase the amount of chaos in the ray trajectories [Couchman et al., Phys. Rev. A 46, 6193 (1992)]. This increase of chaos is expected to reveal itself in the eigenfrequency spectrum as a shift away from Poisson statistics and toward the Gaussian orthogonal ensemble (GOE) statistics of random matrix theory. Numerical results are presented that confirm the predicted shift in the spectral statistics. [Work supported by ONR and DOE.]

\section{$4 \cdot 30$}

4pSA8. Statistical measures of transfer functions in complex structures. Richard G. DeJong (Eng. Dept., Calvin College, Grand Rapids, MI 49546)

The frequency response function of structures can be modeled with pole-zero functions or as modal summations. Either representation can be used to obtain probability distributions for the magnitude of the frequency 
response functions. These probabiltiy functions range from trigonometric functions for simple structures to log-normal distributions for complex structures. Examples are given for simulated and measured frequency response functions of beams, plates, and vehicle structures. The statistical measures obtained from frequency averages are similar to those obtained from ensemble averages in complex structures. This suggests that a sufficiently complex structure can be considered ergodic in the frequency domain.

THURSDAY AFTERNOON, 30 NOVEMBER 1995

ROSE GARDEN, 1:00 TO 4:00 P.M.

\title{
Session 4pSC
}

\section{Speech Communication: Current Directions in Vowel Perception Research (Poster Session)}

\author{
Mitchell S. Sommers, Chair \\ Department of Psychology, Washington University, Campus Box 1125, St. Louis, Missouri 63130
}

\section{Contributed Papers}

\begin{abstract}
All posters will be on display from 1:00 p.m. to 4:00 p.m. To allow contributors an opportunity to see other posters, contributors of odd-numbered papers will be at their posters from 1:00 p.m. to 2:30 p.m. and contributors of even-numbered papers will be at their posters from 2:30 p.m. to 4:00 p.m. To allow for extended viewing time, posters will remain on display until 8:00 a.m. on Friday moming.
\end{abstract}

4pSC1. The relation between identification and discrimination of vowels by young normal-hearing and elderly hearing-impaired listeners. Maureen P. Coughlin, Diane Kewley-Port, and Larry E. Humes (Dept. of Speech and Hearing Sciences, Indiana Univ., Bloomington, IN 47405)

Four young normal-hearing ( $\mathrm{YNH}$ ) and four elderly hearing-impaired subjects (EHI) with moderate sloping sensori-neural hearing losses participated in vowel-identification and formant-discrimination tasks. To examine the relationship between vowel-identification and formantdiscrimination abilities in conditions differing in audibility, signals were presented at two levels (70 and $95 \mathrm{~dB}$ SPL). Four mid-vowels $(/ \mathrm{t} /, \mathrm{e} /, / \varepsilon /$, and $/ \nsubseteq /$ ) were chosen as the target signals for both tasks. Identification performance for the $\mathrm{YNH}$ subjects was near ceiling performance. The EHI subjects averaged $80 \%$ for the $95 \mathrm{~dB}$ and $69 \%$ for the $70 \mathrm{~dB}$ SPL presentation level, although individual subject variability was high. Equivalent discrimination performance in the $F 1$ region ( $\Delta F$ threshold approximately $30 \mathrm{~Hz}$ ) was observed for all four vowels, between groups and across levels. In the $F$ region the EHI subjects' thresholds were elevated compared to the YNH subjects at both levels, even when the formants appeared to be fully audible (at $95 \mathrm{~dB}$ SPL). Correlational analyses suggested that vowel identification was partially predicted by reduced ability to discriminate spectral differences in the $F 2$ region (at higher frequencies) as well as the subjects' hearing loss. [Work supported by NIH and NIA.]

4pSC2. Identification of natural and synthesized vowels produced by children and adults: Effects of fundamental frequency variation. William F. Katz, Peter F. Assmann, and Kathleen M. Jenouri (Univ. of Texas, Callier Ctr. for Commun. Disord., 1966 Inwood Rd., Dallas, TX 75235)

Fundamental frequency $(F 0)$ and formant frequencies $(F 1-F 4)$ were measured for vowels from $\mathrm{hVd} /$ words produced by 10 men, 10 women, and 30 children (ages $3,5,7$ ). For all age groups, intrinsic $F$ (IF0) differences were present, i.e., high (open) vowels had higher $F 0$ 's than low vowels. However, there was increased $F 0$ variability for younger children. Theoretically, the vocal tract transfer function is less precisely defined in the vowel spectrum when $F 0$ is high. Therefore, time-varying changes in $F 0$ could help delineate the shape of the transfer function. Also, IF0 might help specify vowel identity. To determine the perceptual consequences of F0 variation, natural and synthesized versions of a subset of the vowels were presented to listeners for identification. Although the natural vowels were identified more accurately than the synthesized vowels, there was no reduction in accuracy when the natural $F 0$ contour was held constant over the time course of the vowel. Moreover, accuracy was not reduced when IF0 differences were eliminated by synthesizing each vowel with the speaker's average $F 0$. These results suggest that $F 0$ variation does not play a prominent role in vowel identification for isolated syllables produced by children and adults. [Work supported by Texas Advanced Research Program.]

4pSC3. Identification of natural and synthesized vowels produced by children and adults: Effects of formant frequency variation. Peter F. Assmann, William F. Katz, Kathleen M. Jenouri, and Phillip W. Hamilton (School of Human Development, Univ. of Texas at Dallas, Box 830688, Richardson, TX 75083-0688)

To examine developmental patterns in the production and perception of American English vowels, recordings were made of $12 \mathrm{hVd} /$ words from 10 men, 10 women, and 30 children (ages $3,5,7$ ). Fundamental frequency $(F 0)$ and formant center frequencies $(F 1-F 4)$ were estimated and a subset of the measurements served as input to a cascade formant synthesizer. Natural and synthesized vowels were presented to adult listeners for identification. Overall, natural tokens were identified more accurately than synthesized versions. Performance was significantly lower when timevarying changes in either $F 1$ or $F 2$ were replaced by constant values drawn from the vowel nucleus. A further drop in accuracy resulted when all formants $(F 1-F 4)$ and $F 0$ were "flattened," consistent with findings of Hillenbrand [J. Acoust. Soc. Am. 97, 3245(A) (1995)]. These findings highlight the perceptual importance of time-varying changes in vowel spectra. It has been suggested that time-varying changes in the formants can improve the intelligibility of vowels whose spectral envelopes are sparsely sampled by harmonics of the source spectrum. Although the vowels produced by children were generally less well identified, there was no evidence of an increased contribution of formant frequency dynamics with decreasing age. [Work supported by Texas Advanced Research Program.]

4pSC4. Creation of a children's speech database. James D. Miller, Sungbok Lee, Rosalie M. Uchanski, Amold F. Heidbreder, Barbara B. Richman (Central Inst. for the Deaf, 818 S. Euclid, St. Louis, MO 63110), and John Tadlock (Technology Resources, Inc., St. Louis, MO 63141)

Speech was recorded from a large number of children ages 5 through 18 years inclusive, at a custom installation at the St. Louis Science Center. Two separate sets of recordings were made: (1) using a high-fidelity microphone, and (2) using one of five telephone handsets. To date, 415 and 456 children have been recorded for each data set, respectively. Microphone recordings are two repetitions of 15 American English vowels in 
/stop/-V-/stop/ words embedded in a carrier sentence. Five additional English sentences were also recorded twice each. The vocabulary for the telephone recordings included (1) 25 commands (e.g.. delete, new name) spoken twice each, (2) six telephone numbers, and (3) spontaneous answers to eight questions. Exact distributions of the number of talkers of each age and sex will be presented. The recording environment, procedures, and real-time digitization equipment will be described and illustrated. [Work supported by Technology Resources, Inc. and Central Institute for the Deaf.]

4pSC5. Acoustic indices of production mechanisms underlying tonal "grunt" calls in baboons. Michael J. Owren (Dept. of Psych., Reed College, 3203 SE Woodstock Blvd., Portland, OR 97202), Robert M. Seyfarth, and Dorothy L. Cheney (Univ. of Pennsylvania, Philadelphia, PA 19104)

Free-ranging baboons (Papio cynocephalus ursirus) produce brief, tonal "grunt" calls in a variety of social circumstances. These calls bear a striking resemblance to human vowels, being composed of regular, apparently harmonic energy forming several prominent energy peaks below 5 $\mathrm{kHz}$. This resemblance was tested by examining 216 grunt calls from nine adult female baboons for clues to the mechanisms apparently involved in their production. Comparisons of spacing of purported harmonics in grunt frequency spectra to the results of other pitch-extraction methods strongly supported the supposition that these calls are produced using regular vocal fold vibration at approximately the same rates found in adult humans. Examination of individual grunt waveforms showed variation in apparent modes of vibration corresponding to human phonation in the modal and pulse registers. LPC analyses revealed an overall spectral pattern approximating that of the vowel 2 . However, formant positions were found to vary to a greater degree among calls produced by different individuals than between communication contexts. This outcome suggests a low degree of flexibility in articulator positioning. Overall, baboon grunts acoustic features appear to reflect the action of a source-filter production system in which vocal tract filtering mainly provides cues to caller identity.

4pSC6. Animal and computational models of development of graded vowel categories. Andrew J. Lotto, Keith R. Kluender, and Lori L. Holt (Dept. of Psych., Univ. of Wisconsin, 1202 W. Johnson St., Madison, WI 53706)

Some acoustic instances of phonetic segments are phonemically more compelling than others, and studies involving human infants and monkeys suggest that experience plays a critical role in modifying the manner in which subjects respond to between and within phonetic category differences. In this study, eight European starlings (Sturnus vulgaris) were trained to discriminate vowel tokens drawn from stylized distributions either of the Engish vowel categories /i/ and /I/, or of the Swedish vowel categories $/ y /$ and $/ \mathrm{t} /$. Following training, responses to novel stimuli drawn from these distributions indicated that starlings' responses generalized with facility to novel category examples. Multiple linear regression analyses revealed that responses could be well predicted on the bases of $F 1$ and $F 2$ values and distributional characteristics of experienced vowel sounds. Responses manifested a graded structure about the central "prototypical" vowel of the training category. Starling response rates (/i-1/birds) corresponded closely to adult human judgments of "goodness" for the English vowel sounds. Finally, a simple linear association network model trained with vowels drawn from the training set captured $95 \%$ of the variance in birds' response rates for novel vowel tokens. [Work supported by NIDCD and NSF.

4pSC7. Hot spots in the newborn vowel space. Michelle A. Aldridge (Callier Ctr. for Commun. Disord.-UTD, 1966 Inwood RJ., Dallas, TX 75235), Katrena L. Kneeppel, and T. Bower (Univ. of Texas at Dallas, Richardson, TX 75803)

The vowels $/ \mathrm{i} /, / \mathrm{y} /, \mathrm{u} /$, and /unrounded $\mathrm{u} /$ detine one surface of the vowel space. whether defined in articulatory or acoustic terms. Adults can give goodness ratings to vowels from the space surrounding prototypic vowels, the prolotypic vowels typically obtaining the highest ratings. Does this reflect unleamed structural sensitivities or some averaging process during life? Is it true in newborns? Attractiveness or "goodness" was measured by how long newborns listened to first presentation of a set of vowel stimuli. The longer the listen, the better the newborn perceived that vowel to be. The edges of the surface defined by $/ \mathrm{i} /, / \mathrm{y} /, \mathrm{k} /$, and /unrounded $u /$ was broken into 40 steps. Twenty newborns (7-33 h) were presented with 21 stimuli defining two edges of the vowel surface, five infants begining at each corner of the vowel surface. The comer, prototype vowels are preferred over intermediares; even / $y /$ and /unrounded $u /$ which do not occur in Texas. However, $/ i /$ and $/ \mathrm{L} /$, which do oecur in Texas, do elicit more listening than the other two corner vowels. Unlearned, structural sensitivities have been affected by experience in infants as young as $7 \mathrm{~h}$ of age.

4pSC8. Competition and categorization: The effect of choice set on vowel category structure. D. A. Galama and Terrance M. Nearey (Dept. of Ling., Univ. of Alberta, 4-32 Assiniboia Hall, Edmonton, AB T6G 2E7, Canada)

The effect of competition on the perception of category peaks and on goodness judgments was examined. A 48-step vowel continuum spanning the /I-E-æ/ range was synthesized by varying $F 1$ and $F 2$. Subjects listened to multiple presentations of all 48 vowel stimuli in five separate conditions, with nature of the choice set as the dependent variable. The five choice sets were: $/ I-\varepsilon-æ /, / I-\varepsilon /, / \varepsilon-æ /, / I-x /$, and $/ \varepsilon /$. For each of the first four conditions, subjects were asked to simultaneously categorize and provide category goodness ratings for the vowel stimuli. In the fifth condition, subjects were required to provide goodness judgments only, thereby indicating perceived degree of membership to the category $/ \varepsilon /$. Identification and goodness functions corresponded well to each other, with changes in the competition paradigm affecting both of these functions similarly. Also, location of category peaks and range of goodness judgments exceeding $90 \%$ were found to remain relatively stable, regardless of the number of categories in the choice set. While the results of various normalization studies suggest that the structure and location of vowel category peaks is highly affeeted by various contextual factors, they appear to be relatively unaffected by the nature of category competition. [Work supported by SSHRC.]

4pSC9. Study of quantal theory of vowel production using area function and articulatory models. T. V. Ananthapadmanabha and Megha Sundara (Voice and Speech Systems, 53, "Girinivas," Temple St., Malleswaram, Bangalore 560003 , India)

Quantal theory of Stevens [J. Phonetics 17. 3-45 (1989)] states that there appear to be ranges of the articulatory parameter for which there is very little change in the acoustic parameter and other ranges where the acoustic parameter is more sensitive to changes in articulation. This result has been arrived at using a three- or four-fube model and coupled resonator theory. In this paper, the above experiment is replicated using a more realistic frequency domain method for calculating the formant frequencies for a given area function which include the effect of yielding walls and radiation impedance. Further, the quantal theory of vowel production is studied using Steven's and Mermelstein's articulatory models. Nomograms have been generated for these models. The acoustic and perceptual effects of perturbing articulatory parameters centered around the target values for selected vowels are studied. Preliminary experiments show that (a) the so-called areas of stability (broad $F 2$ maximurn, $F 3$ minimum) are, in fact, most sensitive for articulatory changes and (b) for a given change in articulation, while $F 2$ is insensitive, $F 1$ and/or $F 3$ are highly sensitive. In view of these findings, the quantal theory may have to be restated.

4pSC10. Vowel recognition using an articulatory representation. $G$. Richard, Q. Lin, F. Zussa, D. Sinder, C. Che. and J. Flanagan (Rutgers Univ.-CAIP Ctr., Piscataway, NJ 08855-1390)

An alternate approach to speech recognition based on an articulatory representation of speech is proposed. Unlike traditional methods based on Fourier or cepstrum representations, the articulatory description of speech provides a compact parametrization linked to physiological properties. The expected results are robust speaker-independent speech recognition. In fact, physiologically related parameters should be useful for handling variability across speakers. In this work, a three-parameter aniculatory representation is applied to vowel recognition. These parameters (location and size of the main constriction in the vocal tract and the ratio of lip length to mouth aperture) are estimated using a codebook search strategy. Before the 
recognition process, a pattern database is built using a corpus of ten repetitions of nine different vowels. Vector quantization is then applied to this corpus to obtain several representative articulatory vectors, or centroids, for each vowel. In the recognition stage, articulatory parameters of the incoming speech segments are estimated and compared to the centroids of the pattern database to arrive at a recognition decision. Initial results in dicate strong potential for articulatory-based speech recognition. [Research supported by ARPA-DAST 63-93-C-0064.]

4pSC11. Back-front classification of English vowels using a cepstrum-to-formant model. Frantz Clermont (Dept. of Comput. Sci., Univ, of New South Wales, Australian Defence Force Acad., Canberra, ACT 2600, Australia) and David J. Broad (Santa Barbara, CA 93105)

A method is described for importing the concept of critical formant spacing into the cepstral domain, with a view toward automatic and robust classification of English vowels into back and front categories. The method is based on a cepstrum-to-formant, linear regression model reported previously [D. J. Broad and F. Clermont, J. Acoust. Soc. Am. 86, 2013-2017 (1989)], which is reformulated in order to transform low-order, linearprediction (LP) cepstra into Bark distances between third $\left(F_{3}\right)$ and second $\left(F_{2}\right)$ formant frequencies. The back-front distinction itself is established using the critical 3.5-Bark distance as a binary decision criterion. The proposed method was evaluated through classification experiments involving (14th-order) LP cepstra and formant frequencies $F_{2}$ (Barks) and $F_{3}$ (Barks), which were all measured from three center frames of nine vowels spoken five times in $/ \mathrm{CVd} /$ context $(\mathrm{C}=/ \mathrm{h}, \mathrm{b}, \mathrm{d}, \mathrm{g}, \mathrm{p}, \mathrm{t}, \mathrm{k} /)$ by four adult, male speakers of Australian English. The accuracies obtained for correct backfront classification range between $94 \%$ and $100 \%$ both on an intra- and an interspeaker basis. These results confirm the effectiveness of relational measures of formant patterns for broad vowel distinction, but more importantly indicate the possibility of deriving certain phonetic features directly from the LP cepstrum.

4pSC12. The long/short vowel distinction in Maori-A preliminary study. Kirk P. H. Sullivan (Dept. Phonetics, Umeå Univ., S-901 87 Umeå, Sweden)

In Măori, vowels are considered to be intrinsically either long or short. Orthographically a long vowel is marked with a macron. The distinction in vowel length is used contrastively and results in word pairs such as matau (to know) and matau (right, as in not left). There are inconsistencies with length marking even internally within Maori language dictionaries [e.g., Williams, A Dictionary of the Maori Language (GP, 1971)]. The long vowels have previously been considered sequences of two identical short vowels [Krupa, Morpheme and Word in Maori (Mouton, 1966)]. The question of what phonetically distinguishes a short vowel from a long vowel and whether these can be distinguished from two consecutive examples of the same short vowel at a morpheme boundary, for example, arises. The current preliminary study aims to examine the durational range of long and short vowels and to determine the factors to be investigated in a longer study. The results relate to one male speaker. Whether a vowel was long or short was marked in the orthographic version of the data by a single speaker. The results are discussed in relation to the factors to be investigated in further studies.

4pSC13. Coproduction, accent, and the tense/lax vowel contrast. Janet M. Fletcher (Dept. of Linguist., Univ. of Melbourne, Parkville, Victoria 3052, Australia) and Jonathan M. Harrington (Macquarie Univ., North Ryde, NSW 2109, Australia)

The kinematics of accented versus unaccented syllables was analyzed in a corpus of Australian English. It was found that unaccented productions of the long vowel /a:/ in the word "barb" show evidence of articulatory truncation. In other words, opening jaw movements into the long vowel are cut short by the jaw-raising gesture for the final $/ \mathrm{b} /$. Unaccented vowels were best modeled as more "coarticulated" or coproduced versions of accented vowels in this corpus. As part of the same experiment, the kinematics of the vowel contrast /a/ vs /a:/ was also examined. Previous studies of Australian English vowels note the acoustic similarity of the vowels in words like "cut" versus "cart." While treated phonologically as part of the set of tense/lax contrasts in Australian English, it is perhaps more accurately described as a vowel quantity as opposed to vowel quality contrast.
Evidence of articulatory truncation or coproduction was also found in three speakers' productions of the first (short) vowel in accented versions of the two words "bub" and "barb." The short vowel was best modeled as a co-produced version of the long vowel. However, there was also evidence of alternative articulatory strategies implying some movement or gesturespecific dynamics in addition to intergestural articulatory timing strategies.

4pSC14. An analysis of Norwegian vowels: What determines the topography of a vowel space? Michael S. Ziolkowski (Dept. of Linguist., Univ. of Chicago, 1010 E. 59th St., Chicago, IL 60637), Joanna H. Lowenstein, Karen L. Landahl, Peter D. Viechnicki, and Richard E. McDorman (Univ. of Chicago, Chicago, IL 60637)

Paucity of information on the acoustic structure of Norwegian and interest in comparing similarities and differences with its well-described neighbor, Swedish, led to an investigation of Norwegian vowels. A commutation set of single-word utterances was presented to 12 native speakers (4 male, 6 female, 2 children), yielding a corpus of 505 tokens. Spectrographic analysis guided measurement of each vowel's first five formant frequencies both at a single point in steady state (single FFT) and throughout (overlapping FFTs) to exploit the varying fundamental frequency through the steady state, which affords a more accurate reading of the formants. Results indicate a pattern of formant convergences and attenuations in the range of the first four formants, a robust finding which falls between the cracks when using a standard $F 1 / F 2$ technique for laying out vowel spaces. That such patterns characterize different vowel categories and dimensions (front-back, rounded-unrounded) opens discussion to more general questions of what determines vowel location in acoustic space [e.g., Lindblom (1986)]. The hypothesis that two languages with isomerous vowel categories will yield unique acoustic topographies when their systems are structured on different primatives/"features" is discussed.

4pSC15. Variability of vowel formant frequencies and speaker normalization: The cases of Mandarin and Taiwanese. Kuo-You Huang (Psych. Dept., Natl. Chung-Cheng Univ., Chia-Yi, Taiwan) and Chin-Hsing Tseng (Natl. Kaohsiung Normal Univ., Kaohsiung, Taiwan)

Formant frequencies of vowels produced by male and female speakers of Mandarin and Taiwanese were measured. Twenty Mandarin-Taiwanese bilingual subjects produced three tokens of each vowel in the $\Lambda \mathrm{V} /$ (for Mandarin) and $/ \mathrm{kV} /$ (for Taiwanese) contexts. Like previous studies with other languages, women's formants were higher than men's in frequency, that high vowels had $F 1$ values lower than low vowels, and that front vowels had $F 2$ values higher than back vowels. Bark-difference measures were obtained from the Bark-transformed formant frequencies; that is $F 0$ was subtracted from $F 1, F 1$ from $F 2, F 2$ from $F 3, F 2$ from $F 4$, and $F 3$ from F4. Two Bark-difference measures (i.e., $F 1-F 0, F 3-F 2$ ) correspond well to vowel height and point of articulation, respectively. Most importantly, high vowels have $F 1-F 0$ values within 3.5 Bark and front vowels have $F 3-F 2$ differences also within 3.5 Bark. These transformations largely reduced the between-speaker variability related to the gender factor. Results of these Mandarin and Taiwanese vowel data agree with English data [A. K. Syrdal and H. S. Gopal, J. Acoust. Soc. Am. 79, $1086-1100(1986)]$ and support the universality of the categoryindependent auditory theory of normalization.

4pSC16. Dynamic information for vowel identity is formant-based, while steady-state information is based on spectral shape. Fred Cummins (Depts. of Linguist. and Cognitive Sci., Indiana Univ., Bloomington, IN 47405)

Recent vowel research has attempted to identify a canonical set of acoustic parameters which best supports vowel categorization. Other work has argued that the speaker-independent information specifying vowel identity is time-varying, rather than static. The present study examines the possibility that these two research issues are related in complex ways. Recurrent neural networks were trained to identify vowels based on one of two types of time series: either spectral-shape (PLP) representations or formant peak ( $F 1, F 2$, and fundamental frequency) representations. Networks were trained using inputs that reproduced the dynamics of vowels excised from continuous speech. The trained networks were then tested with both static and time-varying vowel tokens. Those trained on formant 
information outperformed those trained on spectral shape information. However, when tested on stimuli lacking dynamic information, the formant-based networks suffered more from the absence of time-varying information than did the PLP-based networks. This suggests that timevarying and steady-state information for vowel identity may not share a single "best" representation. [Work supported by ONR.]

4pSCI7. Speech enhancement based on auditory perception. Jun Yang and Zlatan Ribic (Viennatone GmbH, Froebelgasse 26-32, A-1164 Wien, Austria)

It is well known that formants are more important for speech perception than other details of the spectral envelope. Therefore, for the case of speech (vowels) in noise, improving the salience of the formants by decreasing the energy in the valleys between spectral peaks would logically result in improving intelligibility. Furthermore, perceptually only the first three formants are important in determining the sound that is heard, although the higher formants are necessary to produce sounds of acceptable quality. Based on the above, this paper proposes a scheme to enhance the spectral peaks of the first three formants. In this scheme, the first-order high-pass filter with $1-\mathrm{kHz}$ cutoff frequency is used to pre-empliasize the input speech. Three equalizers in parallel form are designed to enhance the amplitudes of the first three formants that are extracted by the cepstral technique and used as the corresponding resonant frequencies of these equalizers. This proposed scheme is particularly effective in the situation where clean speech can be obtained, such as theaters, concert halls, and classrooms with electrical audio-visual aids, etc. The advantages of this scheme over existing schemes will be discussed, and a demonstration tape will also be played during the presentation.

4pSC18. Spectral-envelope synthesis of vowels: Progress report. James D. Miller, Arnold F. Heidbreder, and Sungbok Lee (Central Inst. for the Deaf, 818 S. Euclid Ave., St. Louis, MO 63110)

Synthesizers based on vocal-tract models do not allow independent control of perceptually relevant characteristics of spectral envelopes. The goal is to develop a tool that will allow assessment of envelope characteristics in speech perception by both normally hearing and hearing-impaired listeners. Using LabVIEW (National Instruments, Inc.), a software synthesizer is being developed that is designed to allow systematic and independent control of these envelope characteristics: (1) the locations of up to five spectral peaks; (2) the locations of the minimums (valleys) between peaks: (3) the peak-to-valley depths in decibels; (4) the ratio of peak-to-valley bandwidths; and (5) the overall spectral shape imposed on the patterns of peaks and valleys. The resulting envelopes specify the component amplitudes of carriers consisting of sinusoids. The phases of the component sinusoids can also be individually specified. Additionally, so-called "autofunctions" adjust "nominal" values of the envelope variables in accordance with the pattern of peak locations in a manner designed to be consistent with natural speech. For example, a nominal peak-to-valley depth is increased or decreased based on the logarithmic separation between adjacent peaks. Synthetic vowels and the effects of manipulation of the variables will be demonstrated.

4pSC19. Formant synthesis: Turning cascade into parallel with applications to the Klatt synthesizer. Qiguang Lin and Jingyun Zou (CAIP Ctr., Rutgers Univ., Piscataway, NJ 08855-1390)

Formant speech synthesis is one of the successful approaches for textto-speech synthesis. Usually, formants are connected in parallel for the synthesis of consonants and in either cascade or parallel for vowels. The cascade structure requires a fewer number of control parameters and the synthesized spectrum is guaranteed to be correct. It is best suited for vowel synthesis. However, because of the necessary interchange of a parallel and cascade structure, it is not trivial to maintain good continuity in resonance modes. On the other hand, vowels can also be synthesized by the same parallel formant synthesizer used for consonants. This solution avoids the interchange of resonators, but mone control parameters are necessitated and the resultant spectrum may be distorted. In this paper. a method is described which mathematically turns a cascade configuration into parallel. No additional parameters, that is, the complex amplitudes of formants, are explicitly required. Instead, these parameters are determined from given formant frequencies, bandwidths, and the higher-pole correction. The method thus maintains continuity in resonance modes without requiring extra parameters. The method has been integrated into the Klatt synthesizer. Synthetic speech generated using the proposed method will be demonstrated. [Work partially supported by ARPA Contract No. DAST 63-93C-0064.]

4pSC20. Fractal timing of phonemic transforms. Betty Tuller, Mingzhou Ding, and J. A. Scott Kelso (Ctr. for Complex Systems, Florida Atlantic Univ.. Boca Raton, Fl. 33431)

The focus of the present work is the timing pattern of perceptual change elicited by multiple repetitions of a syllable (the verbal transformation effect; Warren and Gregory, 1958). It is shown that the distribution of the dwell time, the time spent perceiving a given phonemic form before switching to another form, obeys a power law with an exponent valued between 1 and 2. This result is robust, occurring for meaningless syllables and for English words of different initial phonemic salience, and for presentations as short as 1000 syllable repetitions or as long as 10000 repetitions. Thus within this paradign there is no characteristic time scale for perceptual change. [Work supported by NIDCD Grant No. 5-R29DC00411, NIMH Grant No. 5-R01-MH42900, and BRSG Grant No. NSS I-SO7-RR07258.]

4pSC21. The effects of systematic duration changes on phonemic transformations. Magdalene H. Chalikia and Natasha Parvey (Dept. of Psych., Moorhead State Univ., Moorhead, MN 56563)

Previous studies show that listeners who are presented with a repeated sequence of steady state vowels (between 30-100 ms each) report hearing illusory changes in the identity of the speech sounds, a phenomenon called phonemic transformations [M. H. Chalikia and R. M. Warren, Lang. Speech 34, 109-143 (1991)]. The organizations are pretty salient and can be recognized at a later time. In previous studies all vowels were initially at the same duration. In this study, the vowels within a sequence had different durations (baseline stimuli), to resemble characteristics of real speech utterances. The effects of manipulating systematically the duration of individual vowels by increasing or decreasing the duration of each vowel relative to the baseline is investigated. Twenty-six subjects were asked to listen to the baseline stimuli and record what they heard with each. Then, they were asked to match each of the additional vowel sequences to the forms heard with the baseline ones. Matching performance decreased for the short durations (10-60 ms/vowel), but not for the longer ones (40-180 ms/vowel).

4pSC22. Speech variability in the TIMIT database. Demetrios E. Paneras, Nabil N. Bitar, and Carol Y. Espy-Wilson (Elec., Comput. and Systems Eng. Dept., Boston Univ., Boston, MA 02215)

In an effort to understand variability occurring in fairly casual speech, two experiments were conducted using the TIMIT test data which consist of 1680 sentences spoken by 112 males and 56 females. In the first experiment, the TIMIT phonetic transcriptions were compared automatically against the phonemic transcriptions provided in an on-line dictionary. In the second experiment, the TIMIT phonetic transcriptions were mapped to the broad classes vowel, sonorant consonant, stop, fricative, and affricate, and compared to the output of an automatic broad classifier. For the types of variability found, the analysis includes: (1) the contexts in which they occur, (2) the frequency at which they occur, and (3) differences in their manifestations as a function of dialect region. Phonological rules generated from this study are discussed in the context of those cited in the literature. 


\title{
Session 4pSP
}

\section{Signal Processing in Acoustics: Wavelet and Image Processing}

\author{
Edmund J. Sullivan, Chair \\ Naval Undersea Warfare Center, Code 103, Newport, Rhode Island 02841
}

\section{Contributed Papers}

\section{$1: 00$}

4pSP1. Characterization of supersonic projectile shockwaves using wavelets. Brian M. Sadler and Tien Pham (Army Res. Lab., Adelphi, MD 20783)

Wavelets were applied to experimentally obtained acoustic time series for detection and characterization of shockwaves generated by supersonic projectiles. Exploitation of the shockwave ( $N$ wave) for detection is useful in noisy, echo-filled environments. Wavelets generated from the derivative of a quadratic spline yield a wavelet transform (WT) with a deterministic relationship between the WT modulus-maxima and sharp transitions of the signal [S. Mallat and W. Hwang, IEEE Trans. Inf. Theory 38, 617-643 (1992)]. This WT is dyadic in scale but includes all signal shifts. Shockwave detection was accomplished by analyzing across scales. Analysis proceeds from large to small scales because large scales are more low pass and thus have fewer artifacts from high-frequency interference. With a sampling rate of $250 \mathrm{kHz}$ the first seven or eight scales were found to be sufficient for detection and filtering. The $N$-wave produces characteristic double-peak waveforms at the various scales. Cross-scale analysis searches for these waveforms with constraints on $\mathrm{N}$-wave duration. Detection of the leading and trailing edge of the shockwaves was achieved with high accuracy. The algorithm was validated using data from a variety of small (rifle) and large (tank) projectiles.

\section{1:15}

4pSP2. The use of surface wave dispersion for source ranging. Jason T. Penshom and Randall W. Smith (Appl. Res. Labs., Univ. of Texas, 10,000 Burnet Rd., Austin, TX 78713)

Surface wave dispersion curves (velocity versus frequency curves) are used to estimate source range. The data used in this study consist of signals, due to an impulsive source, received on a line array of triaxial seismometers. The first step in the ranging method is the estimation of the surface wave dispersion curve. This is accomplished via the use of the continuous wavelet transform as a time-frequency decomposition. The known dispersion curve is then used in conjunction with the timefrequency decomposition of the received signal to estimate source range. The method and some preliminary results were presented previously [Smith et al., J. Acoust. Soc. Am. 97, 3310(A) (1995)]. This presentation focuses on the effects of receiver separation and signal-to-noise ratio on the ability to accurately estimate the surface wave dispersion curve, and the corresponding effects on the source range estimates.

\section{1:30}

4pSP3. Application of wavelet transform to fluctuation based processing. Jacob George and Ronald A. Wagstaff (Naval Res. Lab., Code 7176, Stennis Space Center, MS 39529)

Recently the topic of wavelet analysis has received considerable attention from physicists, engineers, and mathematicians [C. K. Chui, An Introduction to Wavelets (Academic, New York, 1992)]. One well-known feature of a wavelet transform is the choice of a flexible time window which automatically narrows when observing high-frequency phenomena and widens when studying low-frequency environments, in contrast to a fixed window in traditional Fourier transforms. Alternately, the wavelet transform can be viewed as a two-parameter representation of a signal. These features have been used with advantage in time-frequency analyses [Badiey et al., "Shallow water acoustic/ geoacoustic experiments at the New Jersey Atlantic Generating Station site," J. Acoust. Soc. Am. 96, 3593-3604 (1994); Drumheller et al., "Identification and synthesis of acoustic scattering components via the wavelet transform," J. Acoust. Soc. Am. 97, 3649-3656 (1995)]. Fluctuation based processing which has recently been developed at NRL, takes advantage of the time fluctuations of the signal to improve the signal/noise ratio and to enhance signal detection for several frequencies. The improvements in such processing due to the use of wavelet representation will be discussed. [Work supported by ONR.]

\section{$1: 45$}

4pSP4. A first approximation to wavelet transform. José Romero and Salvador Cerdá (Laboratorio de Acústica, Departamento de Física Aplicada, Facultad de Ciencias Físicas, c/ Doctor Moliner, Burjasot 46100, Spain)

The wavelet transform is a tool usually used to analyze time-varying spectrum signals. In this work a simple algorithm was presented to evaluate the integrals involved in wavelet transform. In the analysis of timevarying spectrum signals different methods are used. One of them is the short-time Fourier transform (STFT). This method was presented and its problems were mentioned. To solve them, the discrete wavelet transform (DWT) and the Weyl-Heisenberg wavelet transform (WHT) were introduced. To compute the DWT an algorithm was presented that replaced the integrals by a sum in analogy to the case of FT, and also permitted computation of the WHT. Some signals were analyzed using three functions as the mother wavelet: the Haar function, the Mexican hat function, and the Morlet function. The analyzed signals were a $1860-\mathrm{Hz}$ tone, a sweep simulated with $\cos \left(a t^{2}+b\right)$, an impulsive signal, and an example on FFT which did not work correctly. Results were graphically represented and comments on every case were realized. It was found that in different cases, it was best to use the mother wavelet functions.

\section{2:00}

4pSP5. The application of wave field dislocations to the characterization of objects using scattered pulsed sound. James $R$. Brewster (Natl. Ctr. for Physical Acoust, Dept. of Phys., Univ. of Mississippi, University, MS 38677)

Wave field dislocations, so named because of their morphological similarity to dislocations in crystals, are singular lines in the phase of a wave. It will be demonstrated that they can be applied to the characterization of sound fields that exhibit a complicated spatial variation. Dislocations have been identified experimentally in wave fields formed by the scattering of pulsed ultrasound from rough surfaces and from the internal microstructure of metals. The statistics of these dislocations can be correlated with the length scale of the features responsible for the scattering. Experiments were performed using pulsed ultrasound, of central frequency $20 \mathrm{MHz}$, 
reflected from the specimen and detected in water immersion using a coincident source-receiver. The phase of the wave is defined in terms of the position of the wave crests in the signal detected as a function of time at a fixed receiver position.

\section{2:15}

4pSP6. Estimation of spatial backscatter power spectra from sonar images. Jules S. Jaffe (Marine Physical Lab., Scripps Inst. of Oceanogr., La Jolla, CA 92093)

Two- and three-dimensional power spectral estimation from sonar backscarter images is an important area of research that has ramifications for understanding the underlying structure of many environmental features. In one case, that of backscatter from groups of animals, the interanimal correlation distances are the inverse Fourier transform of the threedimensional power spectrum. Estimation of three-dimensional power spectra from sonar data is made difficult because of the spreading of the sonar beams as a function of range which results in a lower resolution image. In multidimensional signal processing parlance, the resulting collected sonar data have been convolved with a spatially variant kemel. Standard signal processing methods have difficulty when applied to these kinds of functions, as the usual assumption is that the data have been obtained from a spatially invariant kernel. A method is proposed to estimate the power spectrum of such images by first starting with an ensemble. It is shown how the spatially variant problem can then be converted into a spatially invariant problem. Straightforward methods for power spectral estimation are then used to determine the spectrum. Examples of the use of the methodology on three-dimensional sonar data will be used to illustrate the method.

\section{$2: 30$}

4pSP7. An adaptive threshold target detection and estimation algorithm. Girish Chandran and Jules S. Jaffe (Marine Physical Lab., Scripps Inst. of Oceanogr., Univ. of Calif. at San Diego, La Jolla, CA 92093-0238)

An algorithm to isolate multiple targets reflecting multiple correlated signals arriving at a receiver is described. The algorithm stems from a specific application, an active high-frequency, three-dimensional imaging system that is used to image zooplankton [Jaffe et al., "FTV: A Sonar for Tracking Macrozooplankton in 3-dimensions" (to appear in Deep Sea Research)]. The signals that are transmitted are designed to have flat crosscorrelation properties. The receiver algorithm exploits this property of the transmitted signal set and works by iteratively isolating progressively weaker targets at each iteration step. An important feature of the structure is the ability to adapt the threshold of the detectors of different signals to targets of various strengths. The algorithm leads to a receiver structure which is a modification of the classical parallel matched filter bank to detect targets and estimate their parameters. Experiments were performed in a tank to test the receiver structure. Various objects were suspended in known configurations and then two signals were transmitted at them. The algorithm was successfully able to isolate the targets and estimate their positions and strengths. [Work supported by NSF Grant No. OCE 9421876.]

\section{2:45}

4pSP8. Acoustic imaging with radiation imaging operators. Mohammed I. Raza (Dept. of Elect. Eng., Univ. of Missouri-Rolla, Rolla, MO 65401), Richard E. DuBroff, and Thomas J. Herrick (Uniy. of Missouri-Rolla, Rolla, MO 65401)

A differential operator has been formulated, which can be used to remotely detect boundaries and position of objects from remote data. The operator has been called the radiation imaging operator (RIO). The RIO is composed of local characteristic parameters and local derivatives of the acoustic wave. After operating on the wave distribution, the output gives a minimum at the boundary of the object, when the correct values of the characteristic parameters are used. The RIOs are developed from radiation boundary conditions (RBCs) and the relevant material boundary conditions
(MBCs). The RBCs were formulated [B. Engquist and A. Majda, Math. Comput. 31, 629-651 (1977)] to simulate the propagation of out-going waves without any significant reflection at the truncating boundary. The MBCs are in the form of Dirichlet and Neumann boundary conditions. A finite-difference time-domain algorithm is implemented to image objects using the discretized RIO, where the shape and location of object is not known. The imaging technique has been found to be robust and tolerant to a reasonable amount of noise.

\section{3:00-3:15 Break}

\section{3:15}

4pSP9. Aeroacoustic wideband array processing for detection and tracking of ground vehicles. Tien Pham and Brian M. Sadler (Army Res. Lab., Adelphi, MD 20783)

Wideband array processing using a steered covariance matrix (STCM) [H. Wang and M. Kaveh, IEEE Trans. ASSP 33, 823-831 (1985)] was implemented and evaluated with experimental data from a small baseline aeroacoustic sensor array. The nonstationary character of acoustic signatures from maneuvering vehicles requires harmonic association and adaptive selection of an operating-frequency set for the STCM on a data-block by block basis. The sensor outputs are processed so that all selected frequencies with the same direction of arrival (DOA) have the same rank-one representation in the steered covariance matrix. This matrix was used in the steered minimum variance (STMV) method [J. Krolik, in Advances in Spectrum Analysis and Array Processing. Vol. II, Chap. 6 (Prentice-Hall, Englewood Cliffs, NJ, 1991)] to accomplish adaptive direction finding. Processing gain and corresponding improvement of accuracy in the DOA estimates was observed when compared to narrow-band subspace methods such as ESPRIT. The STMV DOA estimates are more stable and more reliable than the narrow-band methods. This is especially evident in the multivehicle case with a nearby vehicle much louder than more distant ones.

\section{3:30}

4pSP10. Application of narrow-band and broadband signal processing techniques to the acoustic detection and localization of aircraft. Brian G. Ferguson (Defence Sci. and Technol. Organisation, P.O. Box 44, Pyrmont 2009, Australia)

The acoustic spectrum of a transiting aircraft is shown to be "time varying" when received by a stationary observer. This phenomenon is an example of the Doppler effect, which is exploited to localize aircraft having narrow-band lines in their source spectra; for example, propeller-driven aircraft and helicopters. For the present case of a turboprop aircraft, the application of time-varing spectral analysis techniques to the acoustic data from a single sensor enables the instantaneous propeller blade rate to be estimated at short-time intervals during the aircraft transit. The temporal variation of the Doppler blade rate is required as an input to the localization algorithm, which provides estimates of the aircraft's speed and range at the closest point of approach, as well as the source (or rest) frequency of the propeller blade rate. Next, a broadband cross-correlation technique is applied to the acoustic data from a pair of spatially separated microphones to demonstate the automatic detection of an aircraft transit. Short integration times and Doppler compensation are required to track the transit of a fast aircraft.

\section{$3: 45$}

4pSP11. Simulation of real-time recognition of marine-mammal sounds by a multiple-resolution Bayesian classifier. Thomas $J$. Hayward (Naval Res. Lab., Washington, DC 20375-5350)

A multiple-resolution, Bayesian statistical approach to classification [J. Acoust. Soc. Am. 96, 3312(A) (1994)] is tested on simulations of marinemammal sounds received in an ocean waveguide. Training data for several types of marine-mammal vocalizations are obtained from both the Woods 
Hole Oceanographic Institution SOUND database and the Naval Research Laboratory Dual Use Acoustics Center (DUAC) database. Sound samples not in the training set are then presented to the classification algorithm in a simulation of sound emanating from multiple marine-mammal sources [J. Acoust. Soc. Am. 97, 3371(A) (1995)]. This simulation incorporates modeling of ocean acoustic propagation effects on the vocalization waveforms. When combined with event detection, the classification algorithm achieves near-real-time speed in classification of several types of marinemammal sounds. [Work supported by ONR.]

\section{4:00}

4pSP12. Experimental studies of the effect of non-Newtonian constitutivity on propagation of sine waves in liquids. Daniel $R$. Raichel and Ken Takabayashi (Dept. of Mech. Eng., Cooper Union, 51 Astor Pl., New York, NY 10003)

According to the Raichel-Kapfer theory [J. Appl. Mech. $\mathbf{4 0}$ (Series E), 1-6 (1973)], propagation of even the purest sinusoidal sound wave through a fluid will cause higher harmonics to appear, due to the nonlinear nature of fluid motion. Moreover, any deviation of the fluid's constitutivity from Newtonian behavior will cause these harmonics to change in their amplitudes with respect to the fundamental. In the effort to verify these effects, a sonic viscometer was constructed and operated. The device consisted of a 15-cm-diam, 182-cm-long tube that is filled with specimen fluid. A series of pure sine waves, varied octave-wise from $500 \mathrm{~Hz}$ to $8 \mathrm{kHz}$, was introduced through an electromagnetic driver at one end of the tube into distilled water first and then into a $1 \%$ solution of polyethylene oxide which effectively rendered the water non-Newtonian. Fast Fourier transform analyses of the signals intercepted by a hydrophone at the other end of the tube indicate that pronounced changes occur in the second and third harmonics, as predicted by the Raichel-Kapfer theory. [Work supported by the NY State Science Foundation and the Howard Hughes Medical Foundation.]

\section{$4: 15$}

4pSP13. Spectrum space-time signal processing matched with an antenna's motion, medium, and noises. Igor I. Gorban (Inst. of Mathematical Machines and Systems, Natl. Acad. of Sciences, 42 Ave Acad. Glushkov, Kiev, 252187, Ukraine)

There have been many works connected with working out and researching high-quality space-time signal processing (STSP) algorithms matched with medium and noises. However, there has been far less research into the question of STSP algorithms matched with the motion of the anterna in space, especially in the case of the three-dimensional motion of the antenna with variable velocity, angle rotations, and modified form. The aim of this paper is to present the results of research in the field of STSP matched with the complicated motion of the antenna, the medium, and the noises together. This research developed from the author's earlier research that was presented at the conference on Computational Acoustics and its Environment Applications, Southampton, UK, 1995. In this paper the integral equations that describe maximum likelihood STSP matched with the complicated motion of the antenna, inhomogeneous medium, and additive Gaussian space-time correlated noises, have been obtained. Special ways for solving the equations were proposed and high-quality spectrum STSP algorithms were found for partially coherent and stochastic signals. The new and the former algorithms were compared in different conditions. It was found that in many cases the new algorithms were essentially better than the former ones.

\title{
Session 4pUW
}

\section{Underwater Acoustics: Shallow Water Acoustics II}

\author{
Robert J. Cederberg, Chair \\ Naval Research Laboratory, Code 7140, Washington, DC 20375
}

Contributed Papers

1:00

4pUW1. Benchmark cases for range-dependent seismoacoustic propagation codes. Joo Thiam Goh and Henrik Schmidt (Dept. of Ocean Eng., MIT, 77 Massachusetts Ave., Cambridge, MA 02139)

Benchmark solutions exist for range-dependent acoustic propagation in fluid-only media [J. Acoust. Soc. Am. 87, 1497-1545 (1990)]. However, not many reference solutions are available for the case of mixed fluidelastic media. Some simple canonical benchmark problems are presented and the solutions from four different computational methods are compared. They are the elastic PE [J. Acoust. Soc. Am. 93, 1815-1825 (1993)], boundary element method [J. Acoust. Soc. Am. 89, 1629-1642 (1991)], wave-number integration approach [J. Acoust. Soc. Am. 97(A), 3316 (1995)], and a spectral superelement formulation [J. Acoust. Soc. Am. 98, 465-472 (1995)]. Both forward and backscattered results are presented. A common thread in the last three approaches is the use of the SAFARI/ OASES code as a Green's function generator. Conclusions regarding the general performances of these methods are drawn for the various test cases. [Work supported by ONR.]

\section{$1: 15$}

4pUW2. Wave-based extraction of ocean bottom impedance from constant frequency transmission loss measurements. Kevin D. LePage (Bolt Beranek and Newman, Inc., 70 Fawcett St., Cambridge, MA 02138)

One method of characterizing the propagation in a shallow-water region is to collect a series of measurements which together form a composite of the range-dependent transmission loss (TL) between a source and receiver at single frequencies. Here, a wave-based method of extracting the bottom impedance matrix is presented. As opposed to traditional approaches to extracting environmental information from these measurements, where the bottom properties are perturbed until the modeled and the measured transmission loss agree, the wave-based method is a two-part method, where the horizontal wave numbers of the waveguide are first determined such that all the features in the measured TL are accounted for, and then the water column properties are used in conjunction with a finitedifference scheme [M. B. Porter and E. L. Reis, J. Acoust. Soc. Am. 77, 1760-1767 (1985)] to determine the bottom impedance at these wave numbers. One attraction to this approach is that most features in the data are modeled, and all the unique information about the bottom is contained in an impedance matrix over horizontal grazing angle and frequency. Since 
many of the unknown physical processes which affect shallow-water transmission are concentrated in the bottom, modeling issues are reduced by this approach.

\section{$1: 30$}

4pUW3. Short-range seismoacoustic propagation on and off the beach. LeRoy M. Dorman, Allan W. Sauter, Chris Bradley, Sean Wiggins, and Javier Porras (Marine Physical Lab., SIO, UCSD, La Jolla, CA 92093-0215)

Cultural seismic noise (noise caused by human activities) can be used for tracking and surveillance. During June and July 1995, a field experiment, dubbed "Adaptive Beach Monitoring" was conducted on both sides of the shoreline at U.S. Marine Corps Base Camp Pendleton, near Oceanside, California. Various sources were observed by seafloor seismic and acoustic sensors (four ocean-bottom seismometers), and by a 24-element seismometer array ashore. Preliminary results on the propagation of surface (and interface) waves shows that the surficial shear velocity is similar on both sides of the shoreline (nominally $250 \mathrm{~m} / \mathrm{s}$ ). The dispersion of the waves is, however, markedly different. The dispersion of Scholte waves observed on the OBSs was strong, with group velocity varying by a factor of 2 in the 2- to 10- $\mathrm{Hz}$ range. Ashore, however, Rayleigh waves in the 5to $20-\mathrm{Hz}$ frequency range showed little or no dispersion. [Work sponsored by the Office of Naval Research, Code 32.]

\section{$1: 45$}

4pUW4. Extracting modal structure from vertical array ambient noise data in shallow water. Paul Hursky (Lockheed Martin Aeronautical Systems, San Diego Office, 52990 Gatchell Rd., San Diego, CA 92152-7314), William A. Kuperman, and William S. Hodgkiss (Scripps Inst. of Oceanogr., San Diego, CA 92093-0701)

Measurements of the acoustic mode structure in shallow water can be inverted to determine ocean environmental acoustic parameters. This modal stucture is embedded in shallow-water ambient noise [Kuperman and Ingenito, J. Acoust. Soc. Am. 67, 1988-1996 (1980)]. Ambient noise measurements (SWellEx 1) using a vertical array spanning the lower duct of a summer sound-speed environment were used to extract the acoustic modal structure of a coastal water environment off southern California. The experimental results derived from eigenvector processing are consistent with simulations using the measured environmental parameters.

\section{2:00}

4pUW5. Applications of optimized rational approximations to parabolic equation modeling. R. J. Cederberg, Michael D. Collins (Naval Res. Lab., Washington, DC 20375), and William L. Siegmann (Rensselaer Polytechnic Inst., Troy, NY 12180)

Rational approximations designed using least-squares constraints are easy to obtain and have useful applications. The coefficients are defined in terms of accuracy constraints on the propagating part of the spectrum and stability constraints on the rest of the spectrum. Among the applications are a filtering operator as well as improved approximations for the split-step Padé solution [J. Acoust. Soc. Am. 93, 1736-1742 (1993)] and radiation boundary conditions for outgoing wave equations [Clayton and Engquist, Geophysics 45, 895-904 (1980)]. An efficient operator filter is obtained by designing a rational approximation that is the identity function (or a weighted function) over the propagating (or desired) spectrum and decays to zero elsewhere. Operator approximations have previously been applied to replace the operator in the interior of the domain (the parabolic wave equation), generate radiation boundary conditions, solve scattering problems, and generate initial conditions. With the improved approximations, it is possible to use range steps of more than a hundred wavelengths with the split-step Padé solution. By enforcing both accuracy and stability constraints, it should be possible to overcome stability problems [Howell and Trefethen, Geophysics 53, 593-603 (1988)] and obtain useful higher-order radiation boundary conditions for outgoing wave equations.
4pUW6. Geoacoustic profile estimation by inversion of head wave data. N. Ross Chapman (School of Earth and Ocean Sciences, Univ. of Victoria, Victoria, BC V8W 2Y2, Canada) and David E. Hannay (JASCO Res. Ltd., Victoria, BC V8M IP7, Canada)

An approach is described for estimation of geoacoustic model parameters in shallow water based on inversion of head wave data obtained in experiments with vertical line arrays (VLA). Analysis of head waves obtained in seismic refraction surveys with horizontal arrays is a widespread practice in exploration seismology. However, in shallow water, there can be practical advantages in the use of VLAs. Inversion of head wave data obtained with a VLA provides estimates of the layer depths, and compressional wave speeds and attenuations. Three inversion techniques are compared: inversion of travel time versus range data for a single sensor, direct measurement of the critical angle using the VLA, and inversion of travel time versus hydrophone depth data for a specific range. The techniques are applied to data from an experiment on the continental shelf off Vancouver Island. Shallow explosive charges were used as sound sources out to ranges of $5 \mathrm{~km}$. Strong head wave signals were recorded from two distinct layers, and the data were inverted using the travel time versus depth method. The estimated values of 1750 and $1900 \mathrm{~m} / \mathrm{s}$ for the compressional wave speeds are consistent with results from conventional seismic surveys in the vicinity.

\section{$2: 30$}

4pUW7. Bathymetrically refracted modal eigenrays and bottom attenuation: A paradoxical relation. B. Edward McDonald (Naval Res. Lab., Washington, DC 20375)

Shallow ocean acoustic propagation generally results in bathymetric refraction toward deeper water, and is accompanied by bottom attenuation. In modal eigenray calculations involving small horizontal grazing angle interactions with bathymetry, cases have been found [Heaney et al., J. Acoust. Soc. Am. 90, 2586-2594 (1991)] in which path integrated bottom loss actually decreases with increasing mode number. This apparent paradox is resolved by showing that the increase in modal ray turning rate with mode number can exceed the accompanying increase in bottom attenuation rate. Thus fixed angular deflection can imply attenuation which decreases with mode number. Investigation of acoustic eigenmodes leads to an algebraic relation between bathymetrically induced rates of horizontal ray turning and modal energy loss. This relation gives the modal attenuation rate per increment of horizontal ray turning, and is expressed in $\mathrm{dB}$ per radian. The $\mathrm{dB}$ per radian relation is in general not a monotonic function of mode number. Implications of this result may be relevant to observations in the Heard Island Feasability Test. [Work supported by NRL and Scripps Institute of Oceanography.]

\section{2:45}

4pUW8. Acoustic properties of marine sediments in the North West Shelf of Australia. P. Philip Thomson and John I. Dunlop (Dept. of Appl. Phys., School of Phys., Univ. of New South Wales, Sydney 2052, Australia)

Mathematical models for characterizing the propagation of acoustic waves in shallow water require knowledge of such acoustic properties as dilatational velocity, attenuation constant, shear velocity, and attenuation of the seafloor. In situ measurement of these properties is difficult due to the remoteness of the sea bottom. There are uncertainties in predicting these properties from geological features such as porosity, grain size, density, etc., and there is a need for direct measurements. This paper outlines some exploratory work on the laboratory measurement of core samples taken from the North West Shelf of Australia and subsequent mathematical modeling to predict general propagation characteristics. The sound-speed ratio and attenuation constant were measured by timing a high-frequency wave packet through a length of sediment core. Shear wave measurements were made using a similar measurement frame with piezoceramic bender disk transducers of $\mathrm{I}-$ to $2-\mathrm{kHz}$ resonance frequency. Before making mea- 
surements, the samples were individually evacuated in a mild vacuum for a short period and then slowly infused with seawater at room temperature. Measurements were made at three different positions in the cores corresponding to different depths.

\section{3:00-3:15 Break}

\section{3:15}

4pUW9. Experimental measurements of three-dimensional underwater sound propagation over a variable bathymetry laboratory scale model. Joseph M. Riley, Stewart A. L. Glegg (Ctr. for Acoust. \& Vib., Florida Atlantic Univ., Dept. of Ocean Eng., Boca Raton, FL 33431), and Grant B. Deane (Scripps Inst. of Oceanogr., La Jolla, CA 92093)

Experimental measurements have been conducted giving a complete survey of the acoustic field from a point source in a three-dimensional shallow-water environment. The measurements have been conducted over a 1/10 000th scale model of the Santa Lucia Escarpment off the coast of California. The results of the experiment show three-dimensional propagation effects and areas of modal cutoff in the shallow-water regions. In addition, a discontinuity in the acoustic field was observed for propagation over a joint in the model. This observation has implications for localizing changes in seafloor composition from propagation measurements. Numerical predictions of the acoustic field for selected tracks in the survey show good agreement with the experimental results. [Work supported by the Office of Naval Research, Ocean Acoustics Program.]

\section{3:30}

4pUW10. Stochastic inhomogeneous volume model for fine-scale sediment layering. Roger M. Oba (Naval Res. Lab., Stennis Space Center, MS 39529-5004)

An inverse relation of bottom loss to frequency was successfully modeled by random fine-scale sediment layering [C. W. Holland and G. Muncill, J. Acoust. Soc. Am. 94, 1609-1620 (1993)]. A computational method for propagation of the Helmholtz equation in a water column with stochastic volume variation was developed to compute average complex pressure field and average square of the field numerically in a single pass. This model is revised for use in sediment with uncertain layering, assuming negligible shear interaction. A uniform upward refracting sediment has inclusions of fixed thickness, sound speed, and density, but of uncertain vertical spatial distribution. The vertical interlayer spacing is treated as a random variable with gamma distribution. This model also can be used to isolate phenomena associated with density variation versus sound speed variation. [Work supported by the Naval Research Laboratory and the Office of Naval Research.]

\section{$3: 45$}

4pUW11. A numerical investigation of the relative scattering of Biot fast and slow waves by a spherical inclusion in a poroelastic medium. Dennis J. Yelton (Appl. Res. Labs., Univ. of Texas, Austin, TX 78713-8029) and Morris Stern (Univ. of Texas, Austin, TX 78712)

The relative scattering of Biot fast and slow wave pulses by a spherical inclusion in a poroelastic medium is computed by applying the single frequency steady-state solution of Zimmerman and Stern [J. Acoust. Soc. Am. 94, 527-536 (1993)] to the discrete Fourier components of an incident pulse, and employing linear superposition. The solution of Zimmerman and Stern is an analytical series solution whose convergence is primarily determined by computation precision, which imposes certain limits on the frequency and size of the inclusion. Within these limits, the scattered signals are computed as a function of scattering angle and range for broadband incident fast and slow wave pulses. The findings are compared to experimental observations. [Work supported by the Naval Research Laboratory, Stennis Space Center.]
4pUW12. Determination of seabed statistical properties and their effects on acoustic wave propagation in shallow water. Altan Turgut and Stephen N. Wolf (Naval Res. Lab., Acoust Div., Washington, DC 20375)

Genetic algorithms are used to invert statistical properties of sea bottom/sub-bottom from backscattering data. Backscattering from the seabed is calculated using composite roughness model of Jackson et al. [J. Acoust. Soc. Am. 79(5) (1986)], incorporated with the volume scattering model of Stockhausen [NRE Report 63/9 (1963)]. A parametric study indicates that, for given average sound speed and density of the seabed, bottom roughness parameters such as spectral strength and spectral exponent and sub-bottom parameters such as sound-speed variation, vertical correlation length, and horizontal correlation length can be inverted successfully. Complementary sub-bottom measurement techniques such as core sampling, seismic, and tomographic measurements are also discussed. Acoustic calculations using measured seabed inhomogeneities predict significant coupling to the higher-order modes. This coupling manifests itself as an important loss mechanism.

\section{4:15}

4pUW13. Classification of lake bottom sediments by neural networks using wideband echo signals. Yuanliang Ma (College of Marine Eng., Northwestern Polytechnical Univ., Xi'an 710072, People's Republic of China), Z. Y. Wang (Northwestern Polytechnical Univ.), G. Gimenez, and D. Vray (CREATIS, INSA, Lyon, France)

The paper presents a method of lake bottom sediment classification by artificial neural networks (ANN) using wideband echo signals. The samples to be classified were acquired by experiments at Lake Geneva. There are five types of sediments, namely, silt, rocks, pebbles, sand, and a mixture of sand and gravel. The pattern features are extracted from spectra of echo signals in subband energy expression. Different subband divisions for frequency-domain feature extraction are compared and it is shown that the contant $Q$ method provides better results in comparison with the constant bandwidth method. Using the constant $Q$ method in association with a BP-type locally connected neural network, $85.1 \%$ correct classification in average has been achieved for a testing data set. Wideband echo signals have outstanding superiority for classification in comparison with narrowband signals. It contains more information representing the physical and architectural features of targets. The neural network ultilizes the information through careful optimization and provides a performance improvement up to $10 \%$.

\section{4:30}

4pUW14. Application of a stochastic inversion method for estimating the sound speed in the ocean bottom. Kazuhiko Ohta (5th Res. Ctr., Technical Res. and Development Ctr., Japan Defense Agency, Yokosuka, 239 Japan), Itaru Morishita, Shunji Ozaki, and Tsuneo Ishiwata (Oki Electric Industry Co., Ltd., Tokyo, 108 Japan)

A stochastic inversion method is applied to the problem of determining sound-speed profile in bottom sediment from modal eigenvalues, which can be observed from the FFT beamformed output of a horizontal array in a range-independent shallow-water waveguide. Under a noisy field, each observed eigenvalue has different variance depending on the signal-tonoise ratio (SNR) of modal peaks in the output wave-number spectrum; this difference in SNR originates from the relation between mode shape and source/receiver depths. Thus by using those variances in a covariance matrix involved in stochastic inversion method, it is numerically demonstrated that we can improve the estimation for the solution as compared to that obtained by using a uniform covariance matrix. It is also shown that combination of the same modal eigenvalues observed at the different source or receiver depth can reduce the variance of estimated solution. Application of the stochastic inversion method to real data is also discussed together with comparison with a regularization method. [Work support by JDA.] 


\title{
Meeting of Accredited Standards Committee S1 on Acoustics
}

to be held jointly with the

\section{U.S. Technical Advisory Group for ISO/TC 43 Acoustics and IEC/TC 29 Electroacoustics}

\author{
G. S. K. Wong, Chair S1
}

Institute for National Measurement Standards (INMS), National Research Council, Ottawa, Ontario KlA OR6, Canada

R. W. Krug, Vice Chair S1

Cirrus Research, Inc., 6423 West North Avenue, Suite 170, Wauwatosa, Wisconsin 53213

P. D. Schomer, Chair, U.S. Technical Advisory Group (TAG) for ISO/TC 43, Acoustics U.S. CERL, P.O. Box 4005, Champaign, Illinois 61820

H. E. von Gierke, Vice Chair, U.S. Technical Advisory Group (TAG) for ISO/TC 43, Acoustics 1325 Meadow Lane, Yellow Springs, Ohio 45387

V. Nedzelnitsky, U.S. Technical Advisor (TA) for IEC/TC 29, Electroacoustics

National Institute of Standards and Technology (NIST), Building 233, Room A149, Gaithersburg, Maryland 20899

Standards Committee S1 on Acoustics. Working group chairs will report on their preparation of standards on methods of measurement and testing, and terminology, in physical acoustics, electro-acoustics, sonics, ultrasonics, and underwater sound. Work in progress includes measurement of noise sources, noise dosimeters, integrating sound-level meters, and revision and extension of sound level meter specifications. Open discussion of committee reports is encouraged. The international activities in ISO/TC 43 Acoustics, and IEC/TC 29 Electro-acoustics, will also be discussed. The chairs of the respective U.S. Technical Advisory Groups for ISO/TC 43 (P. D. Schomer), and IEC/TC 29 (V. Nedzelnitsky), will report on current activities of these international Technical Committees.

Scope of S1: Standards, specifications, methods of measurement and test and terminology in the field of physical acoustics including architectural acoustics, electro-acoustics, sonics and ultrasonics, and underwater sound, but excluding those aspects which pertain to biological safety, tolerance and comfort. 


\title{
Meeting of Accredited Standards Committee S3 on Bioacoustics
}

to be held jointly with the

\section{U.S. Technical Advisory Group (TAG) Meetings for ISO/TC 43 Acoustics, IEC/TC 29 Electroacoustics, and ISO/TC 108/SC4 Human Exposure to Mechanical Vibration and Shock}

\author{
T. A. Frank, Chair S3 \\ Pennsylvania State University, Speech and Hearing Clinic, 110 Moore Building, University Park, Pennsylvania 16802 \\ R. F. Burkard, Vice Chair S3 \\ Hearing Research Laboratory, State University of New York at Buffalo, 215 Parker Hall, Buffalo, New York 14214 \\ P.D. Schomer, Chair, U.S. Technical Advisory Group (TAG) for ISO/TC 43, Acoustics \\ U.S. CERL, P.O. Box 4005, Champaign, Illinois 61820
}

H. E. von Gierke, Vice Chair, U. S. Technical Advisory Group (TAG) for ISO/TC 43, Acoustics and ISO/TC 108/SC4, Human Exposure to Mechanical Vibration and Shock

1325 Meadow Lane, Yellow Springs, Ohio 45387

V. Nedzelnitsky, U.S. Technical Advisor (TA) for IEC/TC 29, Electroacoustics

National Institute of Standards and Technology (NIST), Building 233, Room A149, Gaithersburg, Maryland 20899

\begin{abstract}
Standards Committee S3 on Bioacoustics. The current status of standards under preparation will be discussed. In addition to those topics of interest, including hearing conservation, noise, dosimeters, hearing aids, etc., consideration will be given to new standards which might be needed over the next few years. Open discussion of committee reports is encouraged. The international activities in ISO/TC 43 Acoustics, and IEC/TC 29 Electro-acoustics, and ISO/TC 108/SC4 Human Exposure to Mechanical Vibration and Shock, will also be discussed. The Chairs of the U.S. Technical Advisory Groups for ISO/TC 43 (P. D. Schomer), IEC/TC 29 (V. Nedzelnitsky), and ISO/TC 108/SC4 (H. E. von Gierke) will report on current activities of these international Technical Committees and Subcommittees.
\end{abstract}

Scope of S3: Standards, specifications, methods of measurement and test, and terminology in the fields of mechanical shock and physiological acoustics, including aspects of general acoustics, shock, and vibration which pertain to biological safety, tolerance and comfort. 


\title{
Session 5aMU
}

\author{
Musical Acoustics: Steel Pans \\ Thomas D. Rossing, Chair \\ Physics Department, Northern Illinois University, DeKalb, Illinois 60115
}

Chair's Introduction-8:30

Invited Papers

8:35

5aMU1. The history and development of the steelpan. G. Allan O'Connor (School of Music, Northern Illinois Univ., DeKalb, IL 60115)

The history and development of the steelpan is a fascinating story, from its beginnings as a folk instrument in its native Trinidad to a musical instrument played thoughout the world today in calypso, popular, jazz, and classical music. Various steelpans will be demonstrated.

\section{9:05}

5aMU2. Acoustics of steelpans. Thomas D. Rossing, D. Scott Hampton (Phys. Dept., Northern Illinois Univ., DeKalb, IL 60115), and Uwe J. Hansen (Indiana State Univ., Terre Haute, IN 47809)

Steelpans are characterized by strong coupling between note areas partly due to nonlinear vibrational effects. Experimental and theoretical studies of their vibrational behavior will be discussed, and the origin of the rich spectra of harmonic overtones explained in terms of this behavior.

\section{9:35}

5aMU3. Modal analysis and materials considerations in Caribbean steelpans. Uwe Hansen (Phys. Dept., Indiana State Univ., Terre Haute, IN 47809), Felix Rohner (PanArt, Bern, Switzerland), and Thomas D. Rossing (Northern Illinois Univ., DeKalb, IL 60115)

Modal analysis contributes significantly to an understanding of the vibrational patterns and the rich spectral tone color of the Caribbean steelpan sound. This will be illustrated with several examples of computer animations of steelpan normal modes. In addition to showing some of the low-order modes which contribute strongly to the radiated sound, these animations also show direct linear coupling effects between note sections. Since impact excitation modal analysis is fundamentally a linear experimental technique a solenoid driven excitation hammer minimizes amplitude-dependent nonlinearities. PANART artisans have emphasized tonal consistency through material and processing controls. This includes steel production to pan crafters specifications, custom drum manufacture, and a systematic sandwich surface hardening process. A European cultural influence has led to some departure from traditional Caribbean standards. Examples of note spectra and modal analysis computer animations will be shown.

\section{0:05-10:15 Break}

\section{0:15}

5aMU4. Construction and tuning of steelpans. Clifford Alexis (School of Music, Northern Illinois Univ., DeKalb, IL 60115)

During its 50 years of existence, the steelpan has evolved from a folk instrument in its native Trinidad to a versatile instrument capable of performing a wide range of musical styles from symphonic to calypso. Tonal design of the various instruments is approaching standardization but still there is considerable experimentation in the physical design of individual instruments. The construction of the various instruments in the steel drum family is discussed, and the tuning of notes on a cello pan is demonstrated.

10:45

5aMU5. Music of the steelband. Clifford Alexis, Allan O'Connor, and Liam Teague (School of Music, Northern Illinois Univ., DeKalb, IL 60115)

Established in 1973 as the first actively performing steelband in an American university, the NIU Steel Band has toured in the United States and Asia, recorded three albums on disk, cassette, and CD, and has performed with five symphony orchestras, including the St. Louis Symphony and the Chicago Sinfonietta. The program will include classical, popular, and calypso selections, as well as a work composed specifically for them which utilizes an East African Amadinda xylophone. 


\title{
Session 5aPAa
}

\section{Physical Acoustics: Poroelastic Media}

\author{
J. Stuart Bolton, Cochair \\ School of Mechanical Engineering, 1077 Ray W. Herrick Laboratories, Purdue University, \\ West Lafayette, Indiana 47907-1077 \\ Hari S. Paul, Cochair \\ Department of Mathematics, Indian Institute of Technology, Madras, Tamilnadu, Madras 600 036, India
}

Contributed Papers

8:00

5aPAa1. A numerical approach of the transmission of a polyurethane foam sphere in an impulse regime. Abdelkader Sfaoui (Laboratoire d'Acoustique, U.F.R. de Physique, P5, Villeneuve d'Ascq 59655, France)

Among the phenomena envolved in the propagation of an acoustic wave in a diphasic medium such as a polyurethane foam, there is the air/structure interaction. According to the Biot theory the latter is characterized by two parameters: $\rho_{a}$ the inertial coupling and $b$ the resistivity of the fluid flow. When the material porosity, the Young's modulus, and the Poisson's ratio are determined [G. Deprez, J. Phys. Suppl. C2, 43-52 (1990) and A. Sfaoui, J. Acoust. Soc. Am. 97, 1046-1052 (1995)], it is sufficient to adjust these parameters to describe the acoustic propagation. In this aim, an experimental technique has been elaborated. It consists in studying the transmission of a broadband impulse by a foam sphere. The transmission coefficient is obtained after time filtering and fast Fourier transform calculation of two signals: the incident signal and the transmitted signal at the center of the sphere. By selecting the curve to fit the Young's modulus data [A. Sfaoui, J. Acoust. Soc. Am. 97, 1046-1052 (1995)] by means of a regression program, the transmission coefficient is numerically analyzed in the range of $0-20 \mathrm{kHz}$. It follows that the transmitted signal is the superimposition of the Biot fast and slow waves.

\section{$8: 15$}

5aPAa2. The effect of edge constraints on the surface normal impedance of a layer of elastic porous material. J. Stuart Bolton (1077 Ray W. Herrick Labs., School of Mech. Eng., Purdue Univ., West Lafayette, IN 47907-1077), Wim Desmet (Katholieke Universiteit, Leuven, Celestijnenlaan 300B, 3001 Leuven, Belgium), and Nae-Ming Shiau (Ford Motor Co., Dearborn, MI 48121-2053)

Previous work has indicated that the acoustical behavior of partially reticulated noise control foams can be sensitive to small mounting details. It is thus reasonable to expect that the surface normal impedance of a foam sample placed in a standing wave tube will depend on the degree to which the sample is constrained at its edges. Here a two-dimensional version of the Biot theory governing wave propagation in elastic porous materials has been used to investigate the effect of such an edge constraint. First, the allowed modes of propagation within a constrained foam layer were identified. Those modes were then used to predict the response of the constrained layer to an incident plane wave. A comparison of that prediction with the surface normal impedance of an unconstrained half-space of the same material has shown that the principal effect of the edge constraints is to stiffen the sample at frequencies below the cut on of the first shearing mode within the constrained layer. A simple criterion based on the shear stiffness of the elastic porous material has been developed to give the frequency below which the edge-stiffening effect may have a significant effect on a sample's surface normal impedance.

\section{$8: 30$}

5aPAa3. Finite element models for sound transmission through foam wedges and foam layers having spatially graded properties. Yeon June Kang and J. Stuart Bolton (1077 Ray W. Herrick Labs., School of Mech. Eng., Purdue Univ., West Lafayette, IN 47907-1077)

Recently a finite element implementation of the Biot elastic porous material theory has been developed for the purpose of modeling and optimizing partially reticulated foam noise control treatments [Y. J. Kang and J. S. Bolton, J. Acoust. Soc. Am. 98, 635-643 (1995)]. In the present work, that finite element formulation was used first to study normal incidence sound transmission through a foam wedge contained in a hard-walled duct. It has been found that the transmission loss of the wedge is significantly higher than that of a plane foam layer of the same volume in some frequency bands. The increase in transmission loss was found to result from the conversion within the foam of the incident plane wave into a higherorder symmetric mode that does not radiate efficiently from the rear surface of the foam wedge. It has also been found that the same increase in transmission loss can be produced using a plane, constant depth foam layer if the tortuosity is varied across the width (not the depth) of the foam layer. Thus it will be shown that spatially graded lining materials may be used to enhance the transmission loss of double panel systems.

\section{$8: 45$}

5aPAa4. An efficient solution method for solving poroelasticity dynamic problems using the finite-element method. Raymond Panneton and Noureddine Atalla (GAUS, Mech. Eng., Univ. of Sherbrooke, PQ J1K 2R1, Canada)

Recently, a three-dimensional (3-D) finite-element formulation for the dynamic behavior of poroelastic materials was developed [Panneton et al., J. Acoust. Soc. Am. 96, 3339(A) (1994)]. The fluid and solid macroscopic displacements were used as the fundamental variables. The formulation was based on an analogy with 3-D elastic solid elements. However, six degrees-of-freedom (dof) per node were necessary. For large-scale finiteelement models and multifrequency analyses, the use of 6 dof per node has proven to be time- and memory-consuming. Indeed, the complex dissipation mechanisms and frequency-dependent poroelastic coefficients prevent the use of efficient classical solution methods, such as mode superposition method. To alleviate the problem, an efficient solution method is presented. This method is based on assumptions about the poroelastic stress-strain relations and their frequency dependence. A parametric study on the dynamic behavior of poroelastic materials will be presented to back up the used assumptions. Finally, results will be shown to prove that the proposed method leads to substantial gain in computer time without loss of accuracy. 
the tube was determined by applying the modified boundary conditions to

5aPAa5. A model for sound absorption by and sound transmission through limp fibrous layers. Srinivas Katragadda, Heng-Yi Lai, and J. Stuart Bolton (1077 Ray W. Herrick Labs., School of Mech. Eng., Purdue Univ., West Lafayette, IN 47907-1077)

In recent tests it was found that the measured surface normal impedance and transmission loss of limp, fibrous materials could be predicted more accurately when using an elastic porous material model rather than a rigid porous material model: i.e., the motion of the solid phase was found to be acoustically significant. However, it was noted that if the frame stiffness was set to too small a value in the elastic porous model, the boundary value problem that must be solved to yield the surface impedance or transmission coefficient becomes singular. Thus it was of interest to develop a porous material model in which it is assumed from the beginning that the solid phase may move, but that it has no bulk stiffness. Under these circumstances, the Biot model for elastic porous materials may be simplified to yield a single second-order wave equation governing the propagation of a single wave type in the limp porous material. The solutions to that wave equation in combination with appropriate boundary conditions may then be used to predict either the sound absorption by or sound transmission through layers of limp fibrous material without concerns regarding the numerical stability of the predictions.

\section{9:15}

5aPAa6. On the assumption of normal reaction in acoustic characterization of air-filled rigid porous materials. James $\mathbf{M}$. Sabatier, Craig J. Hickey (Natl. Ctr. for Physical Acoust., Univ. of Mississippi, University, MS 38677), and Carl K. Frederickson (Univ. of Central Arkansas, Conway, AR 72035)

The ground has been modeled as a rigid air-filled porous media for the purpose of determining the characteristic impedance important to outdoor sound propagation. It is possible to use these models to acoustically measure the porous parameters of the ground important to agriculture. To completely determine the porous parameters, both reflection and transmission measurements must be made. For both measurements, the ground has been considered to be locally reacting giving transmitted signals that propagate in a direction normal to the surface. This is not nessessarily the case. Using the local reaction assumption, transmission measurements only determine the normal component of the propagation constant. This error lowers the caclulated value of the tortuosity. The error in the tortuosity depends on the angle of incidence of the incident signal. There is no effect on the calculated effective flow resistivity. A theoretical determination of the tortuosity correction will be presented along with data that demonstrate the effect. [Work supported by USDA.]

\section{9:30}

5aPAa7. Reconsideration of sound attenuation in a cylindrical tube due to evaporation-condensation. Yi Mao, James M. Sabatier, and Richard Raspet (Natl. Ctr. for Phys. Acoust., Univ. of Mississippi, University, MS 38677)

Previous results of a theoretical study [J. Acoust. Soc. Am. 96, 3254(A) (1994)] of the sound propagation in a cylindrical tube indicated the sound attenuation due to the evaporation-condensation process underestimated the experimental results in porous materials. Some modifications have been introduced into our theoretical model for solving the discrepancy. First, the fundamental equations in a gas-vapor mixture are resolved including relaxation processes. Again four modes-acoustical, thermal, vorticity, and mass-diffusion modes-are obtained. Second, the temperature on the wall is no longer kept constant; the wall has a finite heat capacity and heat can conduct within the wall. The sound field propagating down a superposition of the newly obtained four modes. The effects of each individual modification on the sound attenuation due to evaporationcondensation are examined. [Work supported by ONR.]

$$
\text { 9:45-10:00 Break }
$$

\section{0:00}

5aPAa8. Acoustic wave processes in viscoelastic porous media. Timothy S. Margulies (Natl. Ctr. for Physical Acoust., Univ. of Mississippi, Oxford, MS 38677)

An understanding of acoustic waves in deformable, porous media has important applications in understanding propagation characteristics through ocean sediments, as well as, in the seismicity of rocks and earthquake design. Continuum balance equations for viscoelastic mixtures are applied to wave dynamics of fluid flow in porous media. Stress representations for the response of the solid phase, for example, Kelvin-Voigt are assumed and compared to frame-indifferent fractional calculus stress tensor assumptions. The linear plane-wave dynamics are developed for homogeneous materials and inhomogeneous layered porous media using matrix propagator techniques. Several specific examples and concluding remarks on possible future directions for theoretical and experimental investigations will be given to gain a better understanding of the effects of coupled deformable solid and fluid motions, in addition to relaxation effects such as chemical reactions or multiphase mass-transfer processes.

\section{0:15}

5aPAa9. Sound speeds and absorptions in a sandy sediment. James M. Sabatier, Christopher C. Lawrenson, James B. Shadwick, Craig J. Hickey, and F. Douglas Shields (Natl. Ctr. for Phys. Acoust., Univ. of Mississippi, University, MS 38677)

A sediment tank $11 \mathrm{ft}$ deep and $10 \mathrm{ft}$ in diameter has been installed at the National Center for Physical Acoustics. This tank has been instrumented with a vertical array of $B \& K 8103$ hydrophones along the axis of the tank, and has been filled with washed "pit run" sand. In studying the sound transmission through the sediment, the hydrophones are used both as senders and receivers. The tank is being used to develop "shaded" array transducers for sub-bottom profiling for measuring sound speeds and absorptions in sediments. Difficulties have been experienced in getting a homogeneous sediment. However, two distinct waves have been observed. The first is a fast wave with a velocity equal to approximately $1600 \mathrm{~m} / \mathrm{s}$ that propagates readily at frequencies up to $100 \mathrm{kHz}$. The second is a much slower wave that cuts off above $2 \mathrm{kHz}$ and has a velocity of a few hundred meters per second. Experiments are under way to determine the frequency dependence off the speed and absorption of these two waves. Calculations are being made in an effort to explain theoretically the experimental results. [Work supported by ONR, MMRI.]

\section{0:30}

5aPAa10. Effect of trapped gas on acoustic properties of model sediments. Mark Wang, F. Douglas Shields, James M. Sabatier, and L. Dwynn Lafleur (Natl. Ctr. for Phys. Acoust., Univ. of Mississippi, University, MS 38677)

Sound speeds and attenuations have been measured in sediments contained in a steel tube 2.5 in. i.d., 3.5 in. o.d., and $5.5 \mathrm{ft}$ long. Measurements have been made in two kinds of sand and three sizes of glass beads over a frequency range of $2-20 \mathrm{kHz}$. In order to study the effect of trapped gases on the acoustical properties of the sediment, a dry sediment was evacuated and then flooded with degassed water. The results are highly sensitive to the packing of the sediment, and are slightly influenced by the pumping process. The measured sound speeds and absorptions after correction for the effects of the yielding wall are compared with those expected from the Biot theory. [Work supported by ONR.] 
5aPAa11. Nonlinear acoustic scattering from trapped gas bubbles in sandy sediments. Frank A. Boyle and Nicholas P. Chotiros (Appl. Res. Labs., Univ. of Texas, P.O. Box 8029, Austin, TX 78713-8029)

A nonlinear backscattering model for trapped bubbles in sandy sediments was developed. A difference frequency scattering coefficient is computed via a technique similar to that of Ostrovsky and Sutin [Nonlinear Sound Scattering from Subsurface Bubble Layers, Natural Physical Sources of Underwater Sound, edited by B. R. Kerman, pp. 363-373 (1993)], which treats the case of bubbles surrounded by water. The Biot theory is incorporated to model the acoustics of a sandy sediment. Biot fast and slow waves are included by modeling the pore fluid as a superposition of two acoustic fluids with effective densities that differ from the pore fluids' actual density and account for its confinement within sediment pores. The principle of acoustic reciprocity is employed to develop an expression for the backscattering strength. [Work supported by ONR under management by APL/UW.]

\section{1:00}

5aPAa12. The detection of objects buried in sand using airborne sound-induced seismic vibrations. James $M$. Sabatier and Tom $\mathbf{M}$ Troutman (Natl. Ctr. for Phys. Acoust., Univ. of Mississippi, University, MS 38677)

Objects buried in air-saturated sand can be detected by measuring the normal component of matrix velocity or acceleration in the vicinity of the object. A loudspeaker positioned $1.15 \mathrm{~m}$ away and $1.15 \mathrm{~m}$ above the surface is driven in the frequency range between 1 and $4.2 \mathrm{kHz}$. Geophones, accelerometers, and an off-the-shelf laser Doppler vibrometer are used to measure the normal velocity component. Surface images of the object are formed by making many point velocity or acceleration measurements with a geophone or accelerometer. An LDV will also be used to produce noncontact, scanned images of small targets in sand. The resolution of this technique is discussed. Also, water-saturated sand will be investigated.
5aPAa13. Dynamic response of axisymmetric pornelastic cylindrical bone. H. S. Paul and V. M. Murali (Dept. of Math., Indian Inst. of Technol., Madras 600 036, India)

The dynamic response of axisymmetric poroelastic bone subjected to transient pressure along the curved surface of the cylinder is studied. In the analysis, exact solutions that satisfy the boundary conditions are obtained by applying Laplace transformations to the equations of motion and fluid flow. The Laplace inversion is obtained from the residue theorem. The stress component $\sigma_{r r}^{*}$ (dimensionless) is plotted with $T$ (dimensionless time) for various $\eta^{*}$ (dimensionless decaying parameter). It is seen that the stresses behave alike for different $\eta^{*}$. In the region $1<T<1.5$, for $\eta^{*}=2$ a slight oscillation is present. The stress component $\sigma^{*}$ (dimensionless) is plotted for the same parameters. Similar behavior is exhibited with the crossing of curves for $\eta^{*}=2$ and 3 . The comparison between the elastic and poroelastic behavior of stress $\sigma_{r r}^{*}$ for $\eta=1$ is carried out. It is seen that there is rapid oscillation for the elastic case, while it is absent in the poroelastic case. For larger time there is no significant effect of stresses. It appears that the elastic constants of material which are considered without porosity may not exist in nature.

\section{1:30}

5aPAa14. Axisymmetric free vibrations of finite poroelastic bone. K. Natarajan and H. S. Paul (Dept. of Math., Indian Inst. of Technol., Madras 600 036, India)

Axisymmetric free vibrations of poroelastic finite cylindrical bone, which behaves as transversely isotropic material, are investigated. Both curved and plane end surfaces of the solid cylinder are free from mechanical stresses and average fluid stresses. Two sets of basic solutions are derived to the equations of motion and poroelastic equation (due to Biot's theory) by applying variable separable technique. From the shear stressfree boundary conditions, eigenvalues for wave numbers are found. Using the basic solutions and eigen wave numbers, solutions to the mechanical displacements and the fluid velocities are developed in series form. The series form solutions satisfy the shear stress-free boundary conditions exactly term by term. Remaining boundary conditions are satisfied by an orthogonalization procedure using trigonometric functions and first kind Bessel functions. Natural frequencies of vibrations are calculated for human bone by varying the number of terms in the series that are tabulated. The series solutions converge rapidly within few terms. For various halflength to radius ratios of the finite cylinder, natural frequencies are computed and presented graphically. [One of the authors (K.N.) acknowledges CSIR, New Delhi, India for the financial support] 


\title{
Session 5aPAb
}

\section{Physical Acoustics: General III}

\author{
David L. Gardner, Cochair \\ Material Division, Los Alamos National Laboratory, MS K764, Los Alamos, New Mexico 87545 \\ Jeffrey R. Olson, Cochair \\ Los Alamos National Laboratory, MS K764, Los Alamos, New Mexico 87545
}

\section{Contributed Papers}

8:00

5aPAb1. Acoustics in the first 100 years of The Physical Review. Robert T. Beyer (Phys. Dept., Brown Univ., Providence, RI 02912)

Recently, the American Physical Society published a volume entitled "The First One Hundred Years of the Physical Review," containing 1200 pages of reprints of "seminal papers." Unfortunately, there are no acoustics papers in that volume. This paper attempts to redress the balance by discussing a number of significant acoustics papers that have appeared in The Physical Review, especially in the years before the appearance of The Journal of the Acoustic Society of America.

\section{$8: 15$}

5aPAb2. Acoustic wave propagation through actin/alpha-actinin gels. Timothy S. Margulies (Johns Hopkins Univ., Baltimore, MD 21218)

The acoustic properties of mixtures of actin filaments and alpha-actinin depend in a complicated way on both the concentration and affinity of the cross-linking protein for actin. The dependence of sound absorption and speed through actin filaments at low frequencies has been analyzed for one-dimensional waves in a viscoelastic medium. Estimates are made using rheology data for the complex modulus versus deformation rate for actin filaments including the effects of various amounts of concentration of alpha-actinin. Calculations of acoustic waves show the influence of the transition from an isotropic viscoelastic solid to a viscous fluid of actin bundles. The perturbative approach for nonlinear wave propagation in viscoelastic media was also applied to investigate gel properties and infuences on waveform modification.

$$
\text { 8:30 }
$$

5aPAb3. Calibrating the methods of stationary phase and steepest descent. Raymond J. Nagem and Brian J. Collins (Dept. of Aerospace and Mech. Eng., Boston Univ., 110 Cummington St., Boston, MA 02215)

Analytic modeling of radiation from sources or scattering by obstacles near submerged layered environments generally leads to Fourier-type spectral integrals with integrands that contain pole and branch point singularities. In the high-frequency range, there are spectral intervals in which the integrands are highly oscillatory, thereby making their numerical evaluation problematic, especially in the vicinity of singularities that lie on the Fourier path in the absence of dissipation. Various methods of performing the numerics are examined, including deformation of the real-axis integration path into a contour in the complex plane, on which the integrand has weak or no oscillations and is exponentially damped away from a local maximum. By comparing the corresponding steepest descent and stationary phase analytic approximations with the numerics, one may calibrate these algorithms for accuracy, especially when saddle (stationary) points and singularities are in close proximity. The physical wave phenomena associated with these altemative analytic models are discussed. Attention is given to the separation of the integrand into weakly and highly oscillatory portions, as required in the analytic approximations.

\section{$8: 45$}

5aPAb4. Edge wave on-axis behind a disk or aperture having a random edge. Pinelopi Menounou, Michael R. Bailey, and David T. Blackstock (Appl. Res. Labs. and Dept. of Mech. Eng., Univ. of Texas, P.O. Box 8029, Austin, TX 78713-8029)

The Helmholtz-Kirchhoff intergral is used to predict the edge wave on-axis behind a disk (or an aperture) that has a ragged edge. The ragged edge is modeled as being made up of $N$ arcs of equal angle (subtended from the center of the disk) but differing radii $r_{i}$. The on-axis edge wave is thus a sum of $N$ scattered signals, each of which has a common amplitude proportional to $1 / N$ but a different delay time $\tau_{i}=\sqrt{r_{i}^{2}+s^{2}} / c_{0}$, where $s$ is the axial distance from the disk. A formula has been derived for the edge wave's rms pressure, in terms of $N$ and the incident wave's rms pressure and autocorrelation function. The formula has been evaluated for incident waves that are sinusoidal, random (noise), and transient. The calculations agree reasonably well with underwater measurements [J. Acoust. Soc. Am. 92, 2359(A) (1992)] made with a spark source and various apertures and disks (biradial, triradial, and ragged). When $N$ is large and the range of values of $\tau_{i}$ is large enough, the rms value of the edge wave approaches zero. [Work supported by ONR, NASA, and the ARL:UT IR\&D program.]

\section{9:00}

5aPAb5. Analysis of the diffraction process near a corner of a rigid wedge. Mitsuhiro Ueda (Intl. Cooperation Ctr. for Sci. and Technol., Tokyo Inst. of Technol., O-okayama, Meguro-ku, Tokyo 153, Japan)

In order to analyze the sound field near a corner of a rigid wedge, the virtual discontinuity principle of diffraction (VDPD) that has been proposed and the representation of the sound field that satisfies both the wave equation and boundary conditions that can be constituted by this principle [J. Acoust. Soc. Am. 95, 2354-2362 (1994)] is applied to this problem. Then the sound pressure on the rigid surface can be expressed as an integral of the particle velocity normal to an extension line of one of the wedge surfaces where a point of interest is located on it. On the other hand, the particle velocity at any field point can be expressed as an integral of the sound pressure along the rigid surface using the Green's theorem. Thus there are two relations between the sound pressure on the rigid surface and the particle velocity normal to the extension lines of the wedge. Analyzing these two relations near the comer of the wedge, the diffraction process that occurs near the corner becomes clear. Its good understanding will surely help in understanding the nature of waves diffracted by a polygon.

\section{9:15}

5aPAb6. Acoustic field in a borehole within a layered formation. Xiuming Wang, Hailan Zhang, and Congfu Ying (Inst. of Acoust., Academia Sinica, Beijing 100080, People's Republic of China)

In acoustic well logging, the borehole may be considered as a vertical waveguide of infinite cross section with horizontal discontinuities, and the conventional mode expansion cannot be applied. Some authors introduced an artificial large coaxial cylindrical boundary, transforming the problem 
into one with finite cross section. However, this approach is limited to low frequency. Based on the theory of generalized function, the mixed spectrum of the infinite cross-section waveguide is analyzed and a new hybrid method is proposed in which the acoustic field is expressed as a sum of several discrete modes and an integral of continuous modes with unknown weight functions which are obtained by numerically solving a group of integral equations deduced from the boundary conditions at discontinuities. It is shown that this method makes it possible to calculate up to the frequency for a typical logging environment. Laboratory experiments have been conducted in which a specific method was adopted to form a liquid cylinder surrounded by fluid layers. Both studies reveal that the reflection of the guided wave at the discontinuity is strongly dependent on the wave frequency. When the frequency is low the reflection is prominent, but at a high frequency the reflection is much weaker.

9:30

5aPAb7. Acoustic radiation force on a rigid sphere in a viscous heat-conducting fluid. Alexander A. Doinikov (Inst. of Nuclear Problems, Bobruiskaya 11, Minsk 220050, Belarus)

This study generalizes earlier calculations by the author [A. A. Doinikov, Proc. R. Soc. London Ser. A 447, 447-466 (1994); A. A. Doinikov, J. Acoust. Soc. Am. 96, 3100-3105 (1994)] to cases where heat conduction of the host fluid and the sphere material is no longer negligible. Three types of an incident sound field were considered: a plane traveling wave, a plane standing wave, and a diverging spherical wave. Effects of heat conduction on the radiation force were found to be greatest for traveling and spherical waves and for spheres less dense than the host fluid. In particular, it is found that traveling waves can urge these spheres away both from and toward the sound transducer and diverging (not standing) spherical waves can cause them to levitate. These results are unusual from the standpoint of the theory of acoustic radiation forces for perfect fluids. [Work supported by the Ministry of Education and Science of the Republic of Belarus.]

\section{9:45}

5aPAb8. Transfer coupler reciprocity method for the absolute low-frequency calibration of field hydrophones under full environmental conditions. Joseph F. Zalesak (Naval Undersea Warfare Ctr., Underwater Sound Reference Detachment, Code 2581, P.O. Box 568337, Orlando, FL 32856-8337)

The reciprocity coupler is a closed chamber used for the absolute calibration of standard hydrophones at low frequencies. There is a need to extend this capability to field hydrophones that are not specifically designed for use in a reciprocity coupler. This requires that one determine the reciprocity parameter for the coupler with the field hydrophone installed. Uncertainties in the effective volume and compliance of the coupler with the field hydrophone installed preclude calculation of the reciprocity parameter from first principles. The transfer reciprocity method described here provides a way to measure the reciprocity parameter of an illcharacterized coupler by first making measurements in a wellcharacterized coupler without the field hydrophone. An error analysis is presented indicating the current state of the existing reciprocity coupler including the transfer reciprocity method. The analysis shows that there is a systematic enror that can be eliminated by correcting the sensitivities obtained using the transfer reciprocity method. This error has a frequencyindependent component of $0.31 \mathrm{~dB}$ and a frequency-dependent component ranging from $0.1 \mathrm{~dB}$ at $700 \mathrm{~Hz}$ to $1.0 \mathrm{~dB}$ at $2000 \mathrm{~Hz}$. After correcting for the systematic error, the uncertainty in the sensitivity is $\pm 0.25 \mathrm{~dB}$ for frequencies below $1000 \mathrm{~Hz}$, rising to $\pm 0.35 \mathrm{~dB}$ for frequencies near 2000 Hz.
10:00-10:15 Break

\section{0:15}

5aPAb9. Measurement microphone calibrations and their uncertainty. Erling Frederiksen (Danish Primary Lab. of Acoust. and Bruel \& Kjær, Transducer Products Div., 2850 Nærum, Denmark)

Today, there is an increasing need for obtaining legal acceptance of and confidence in calibrations performed by laboratories in other countries. This is especially true and clearly observable in Europe where the number of accredited laboratories and calibrations per year are rapidly increasing. To obtain accreditation the laboratories must analyze and state their calibration uncertainty. This leads to focus on uncertainty of microphone sensitivity and sound level calibrations. The overall calibration uncertainty valid for ordinary measurement microphones and pistonphones have been determined by studying error sources and their influence on the calibration results. The uncertainties of the methods, which directly and indirectly influence the uncertainty valid for the user of a Bruel \& Kjær transducer calibration, are discussed. The methods include free-field and pressure reciprocity calibration of refrence standard microphones performed by the Danish Primary Laboratory of Acoustics (operated by Bruel \& Kjær in cooperation with the Danish Technical University, Lyngby) and the electrostatic actuator method which is widely applied by the Bruel \& Kjær Production Department and Calibration Service Centers. Contributions to the uncertainty which are due to the free-field and diffuse-field corrections and to the influence of microphone protection grids will be presented.

\section{0:30}

5aPAb10. Exploring the parameter space of low frequency of sonoluminescence. Joseph C. Jankovsky, Jeffrey A. Ketterling, and Robert E. Apfel (Ctr. for Ultrasonics and Sonics, Yale Univ., New Haven, CT 06520-8286)

An experimental apparatus has been designed to measure the physical properties of low-frequency sonoluminescence (SL) (15 kHz). Radial motion of the bubble has been measured by Mie scattering and also by a new method, which images the bubble through a magnifying $C C D$ camera illuminated by a strobing LED lamp. The light emission of SL is measured by a photomultiplier tube. An automated gas handling system has been constructed in order to measure the effects of various gas mixtures and concentrations on SL. The system consists of voltage actuated valves, a mixing vessel, and pressure sensors, which are interfaced to a computer and controlled using the LabVIEW programming environment. With the lower frequencies and the resulting larger bubbles, it is hoped that the parameter space for this region of SL can be mapped out. [Work supported by .NASA through JPL Contract No. 958722.]

\section{0:45}

5aPAb11. Experimental investigation of onset threshold for shape oscillations for air bubbles in 1g. D. Felipe Gaitan and R. Glynn Holt (Jet Propulsion Lab., 4800 Oakgrove Dr., M.S. 183-401, Pasadena, CA 91109)

Experimental observations of single bubble oscillations have yielded a wide variety of phenomena: periodic and period-doubled spherical oscillations; periodic, quasiperiodic and chaotic multimode shape oscillations; and, most recently, periodic, period-doubled, quasiperiodic, and chaotic sonoluminescence. In an effort to determine the effect of gravity on these phenomena, the acoustic pressure threshold for shape oscillations of single gas bubbles in a $1 \mathrm{~g}$ environment was measured. The bubble sizes varied between 20 and $1005-m$ radius, and were simultaneously levitated and driven acoustically at $20 \mathrm{kHz}$ in water. Mie scattering was used to measure the bubble radius and to monitor its motion before and after the onset of surface instablities giving rise to shape oscillations. A video imaging system was used to determine the mode of the shape oscillations. [Work supported by NASA.] 
5aPAb12. Study of liquid drop shape oscillations with an automatic image analysis system. Robert E. Apfel, R. Glynn Holt, and Yuren Tian (Ctr. for Ultrasonics and Sonics, Yale Univ., New Haven, CT 06520-8286)

Droplet shape oscillations induced by acoustic radiation force can be applied to investigate liquid surface rheology. During experiments performed on the United States Microgravity Laboratory missions, the droplet shape was recorded both by video and by film cameras. In order to determine the free oscillation frequency and damping constant of droplets, an automatic image analysis system was developed to evaluate the variations of droplet shape versus time. With this system the edges of drops are automatically located frame by frame, and then matched by a polynomial function. The geometric center point, the maximum and minimum diameters, and the droplet aspect ratio are calculated from the fitting results, which give the droplet free oscillation frequency and damping constant. From these two parameters and the droplet size, the surface elasticity and viscosity of surfactant solutions can be evaluated. [Work supported by NASA through JPL, contract 958722 .]

\section{1:15}

5aPAb13. A study of the natural frequencies of spherical and distorted bubbles in open cavities. Andrea Prosperetti, Hasan N. Og̈uz, and Jun Zeng (Dept. of Mech. Eng., Johns Hopkins Univ., Baltimore, MD 21218)

The natural frequency of a bubble in an open, liquid-filled cavity is studied. A number of axisymmetric and three-dimensional configurations are considered such as a bubble in a cylindrical tube closed or open at one end and open at the other, a bubble between two parallel plates, a bubble in an open spherical cavity, and others. For bubbles that are smaller than the acoustic wavelength, the incompressible potential flow approximation is adequate. The natural frequency is computed by a boundary integral method. A slightly overexpanded bubble is allowed to relax and oscillate, and its frequency is measured by taking the Fourier transform of the volume pulsations. Alternately, a constant potential over the bubble surface is imposed and the natural frequency is calculated from the resulting volume flow rate of the bubble. These two techniques give nearly identical results for linear oscillations. For each geometry, spherical as well as distorted bubbles are investigated. [Work supported by the Office of Naval Research.]

\section{1:30}

5aPAb14. Calming the capillary waves on the surface of a bubble using an insoluble surfactant. Thomas $J$. Asaki (MS K764, Los Alamos Natl. Lab., Los Alamos, NM 87545), David B. Thiessen, and Phillip L. Marston (Washington State Univ., Pullman, WA 99164-2814)

The excess damping of capillary waves caused by a surfactant monolayer was demonstrated for the quadrupole shape mode of an isolated acoustically trapped bubble in water. A known amount of the waterinsoluble surfactant stearic acid was deposited on the bubble surface from a microliter of ethanol solution. As the bubble dissolves, its surface area decreases thus compressing the monolayer. Compression rates ranged from 0.09 to $0.18 \AA^{2}$ per molecule per minute for different bubbles and surfactant coverage ranged from 20 to $37 \AA^{2}$ per molecule specific area. This technique was tested for accuracy and reproducibility by application to pendant bubbles and subsequent measurements of the static surface tension. Shape oscillations were induced by modulation of the ultrasonic radiation pressure and the free decay was recorded by an optical extinction technique. Local maxima in the normalized damping and frequency were observed near $26 \AA^{2}$ per molecule coverage in agreement with similar studies of capillary waves on flat interfaces. An unexpected increase in the frequency was observed at high coverage and may be associated with the solidification or collapse of the monolayer. Any effects due to the presence of ethanol were shown to be negligible. [Work supported by ONR.]
5aPAb15. Observation of nonlinear scattering of acoustical waves at a subsurface bubble layer. Lev A. Ostrovsky (Univ. of Colorado, CIRES/NOAA Environ. Tech. Lab., Boulder, CO 80303), Zygmund Klusek (Inst. of Hydrodynamics, Gdansk, Poland), Alexander M. Sutin, Irina A. Soustova, Alexander I. Matveyev, and Andrey I. Potapov (Inst. of Appl. Phys., RAS, Nizhny Novgorod, Russia)

To observe nonlinear acoustic effects in a subsurface bubble layer, two echo sounders working at different frequencies were used. The experiments were performed in the shallow area of the southern part of the Baltic Sea aboard the research vessel "Oceania." Two echo sounders were run at 30 and $37 \mathrm{kHz}$; each had a power of about $0.3 \mathrm{~kW}$. The depth of the echo sounders was about $15 \mathrm{~m}$ and the intensity of the acoustic signal in the subsurface bubble layer was enough to observe nonlinear effects. The sum and difference frequency components in the spectra of the scattered signal were determined. A theory of such a nonlinear scattering, based on summarizing the signals scattered by separate bubbles, was developed. It was shown that the sum frequency component was due to a noncoherent reverberation in the bubble layer and the difference frequency component was coherent. This component was generated in the process of primary wave propagation and can be received after sea surface reflection. Experimental and theoretical results have shown that the nonlinear scattering can be used to determine bubble density at sea. [Work was supported by ONR.]

\section{2:00}

5aPAb16. Sonolysis in liquid water without gas bubbles. Dmitry A. Selivanovsky (Inst. of Appl. Phys., 46 Ulyanov St., Nizhny Novgorod 603600, Russia) and Grigory A. Domrachev (Inst. of Metalloorganic Chemistry, Nizhny Novgorod 603600, Russia)

The present paper describes the results of measurements of hydrogen peroxide concentration in water under the action of relatively lowfrequency sound without cavitation. Measurements of hydrogen peroxide concentration have been carried out with a high-sensitivity chemoluminescent method which has been elaborated for these experiments. De-ionized, degassed, high-purity water has been used. Acoustic resonators of a quarter of a sound wavelength were used as vertical columns made from highpurity quartz glass. Resonance frequencies were $1.2,5.6$, and $15 \mathrm{kHz}$. The pressure amplitude in the node at the hard bottom was smaller than 0.6 atm. The formation and accumulation of hydrogen peroxide were observed. For example, for the $20{ }^{\circ} \mathrm{C}$ water temperature and $1.2-\mathrm{kHz}$ frequency the rate of increasing concentration rate is equal to $2 \times 10^{-11} \mathrm{~mol}$ $(/ / \mathrm{h})$ for the degassed water and $8 \times 10^{-10} \mathrm{~mol} /(\ell / \mathrm{h})$ for a solution of magnesium sulfate in degassed water with a concentration similar to the one of the sea. The absence of gas bubbles was controlled by optical and acoustical methods. It is known that the appearance of hydrogen peroxide is a result of dissociation of a part of the water molecules. A hypothesis on the possibility of dissociation of water molecules at a small energy density due to acoustical and mechanical action is discussed.

\section{2:15}

5aPAb17. Sound energy loss during sonolysis. Dmitry A. Selivanovsky (Inst. of Appl. Phys., 46 Ulyanov St., Nizhny Novgorod 603600, Russia) and Grigory A. Domrachev (Inst. of Metalloorganic Chemistry, Nizhny Novgorod 603600, Russia)

This paper gives an analysis of sound energy losses due to sonolysis dissociation of the part of water molecules to radicals $\mathrm{H}$. and $\cdot \mathrm{OH}$. The value of the energy loss can be evaluated by using the concentration of hydrogen peroxide which appears in the water as a result of crossrecombination of radicals $\cdot \mathrm{OH}+\cdot \mathrm{OH}=\mathrm{H}_{2} \mathrm{O}_{2}$. Data previously obtained by different authors were used for the present analysis. Data for fresh water and also for water with dissolved gases were considered. Data covered a sound frequency range from $1.5 \mathrm{kHz}$ to $2 \mathrm{MHz}$ and sound pressure amplitudes $0.6-2.5 \mathrm{~atm}$ for normal static pressure and a water temperature of $20^{\circ} \mathrm{C}$. It is shown that the rate of increasing hydrogen peroxide concentration is proportional to the intensity of sound. The rate is also propor- 
tional to the concentration of dissolved oxygen and argon in water while other gases (hydrogen, helium, nitrogen) do not influence the sonolysis rate compared to the degassed water. All the data demonstrate that the energy loss is about proportional to the square of sound frequency, that is, the same frequency dependence with Rayleigh viscous attenuation of sound. The relalive values of energy losses are from $10^{-4}$ to $10^{-8}$ of the Rayleigh sound decay. This value depends on the kind and concentration of dissolved gas in water.

FRIDAY MORNING, 1 DECEMBER 1995

ST. LOUIS A, B, C, 9:00 A.M. TO 12:00 NOON

\title{
Session 5aSC
}

\section{Speech Communication: Intelligibility, Impaired Populations and Cognitive Factors (Poster Session)}

\author{
Randy L. Diehl, Chair \\ Department of Psychology, University of Texas, 300 Mezes, Austin, Texas 78712
}

\section{Contributed Papers}

\begin{abstract}
All posters will be on display from 9:00 a.m. to 12:00 noon. To allow contributors an opportunity to see other posters, contributors of odd-numbered papers will be at their posters from 9:00 a.m. to 10:30 a.m. and contributors of even-numbered papers will be at their posters from 10:30 a.m. to 12:00 noon. To allow for extended viewing time, posters will remain on display until 5:00 p.m.
\end{abstract}

5aSC1. The effects of speaking rate on the intelligibility of speech for various speaking modes. Jean C. Krause and Louis D. Braida (MIT, Cambridge, MA 02139)

In adverse listening conditions, large and robust increases in intelligibility can be achieved by speaking clearly. The most striking differences between clear and conversational speech are associated with differences in speaking rate. To understand these differences, the intelligibility of speech in a variety of speaking modes was investigated at three different speaking rates. Talkers with significant speaking experience were asked to produce clear and conversational speech at slow, conversational, and quick rates. Previous studies show that the speaking rate for clear speech is roughly one-half that of conversational speech. Therefore, during training, the talkers were given feedback on their intelligibility in order to elicit the clearest possible speech at conversational and quick speaking rates. Talkers also recorded sentences in several other speaking modes such as soft, loud, and conversational with pauses inserted, as required for input to some automatic speech recognition systems. All speech materials recorded were nonsense sentences which provide no semantic context to aid listeners in identifying key words. The effects of changes in speaking rate on intelligibility of various speaking modes for normal hearing listeners in a background of wideband noise are discussed. [Work supported by NIH.]

5aSC2. Speech modulation transfer functions for different speaking styles. Karen L. Payton (ECE Dept., Univ. of Massachusetts, North Dartmouth, MA 02747) and Louis D. Braida (MIT, Cambridge, MA 02139)

The speech transmission index (STI) is highly correlated with speech intelligibility scores when the environment is degraded by noise and/or reverberation and/or the listener's hearing is impaired [e.g., Payton et al., J. Acoust. Soc. Am. 95, 1581-1592 (1994)]. The STl is typically computed from modulation transfer functions (MTFs) that are determined theoretically, based on effective SNR, or on measurements using the RASTI procedure. In principle, however, MTFs can be compuled directly from speech envelope spectra. For the current study, envelope spectra were computed for both conversational and clearly articulated speech. Three environments were considered: quiet/anechoic, reverberant ( $0.6 \mathrm{~s} \mathrm{RT}$ ), and additive noise (0 dB SNR). Results indicate that reliable MTFs can be computed from speech envelope spectra if the coherence function is used to limit the range of modulation frequencies (to reduce the effects of computational artifacts). Also, while MTFs for the two speaking styles are very similar in additive noise, in reverberation MTFs differ as one would expect on the basis of the higher intelligibility of clear speech in reverberant environments. [Work supported by NIH.]
5aSC3. Speech intelligibility of a multiple loudspeaker public address system in long enclosures. Jian Kang (The Martin Ctr., Cambridge Univ., 6 Chaucer Rd., Cambridge CB2 2EB, UK)

Multiple loudspeaker public address systems are often used in long enclosures, such as underground stations, where one dimension is much greater than the other two. Classic room acoustic theories cannot be used in long enclosures as the assumption of a diffuse sound field is not satisfied. In this research a theory of reverberation in long enclosures is established [J. Kang, Acustica (to be published)]. It is shown that the reverberation in long enclosures varies systematically with the source-receiver distance. Based on the theory, a computer program for calculating the speech transmission index (STI) of multiple loudspeakers in long enclosures was developed, with which the optimal loudspeaker directionality and spacing can be determined. With the help of this program, a series of treatments for improving the speech intelligibility of multiple loudspeakers were investigated in a 1:16 scale model of St. John's Wood station in London. It was found that the STI can be improved effectively by diffuse treatments and strategic positioning of absorption. Some of the above results are being used for the new Hong Kong airport-underground project. [Work supervised by Dr. R. J. Orlowski and supported by MTRC, ORS, and COT:]

5aSC4. Intelligibility of normal speakers: Error analysis. Amy T. Neel (Speech Res. Lab., Dept. of Psych., Indiana Univ., Bloomington, IN 47405)

Errors made by listeners in sentence transcription were analyzed to determine their contributions to intelligibility. One hundred Harvard sentences produced by ten males and ten females were transcribed by ten listeners per talker. Two measures of intelligibility were obtained: a key word score in which a sentence was correct if, and only if, all five keywords were correct; and a total error count. Analysis of error types revealed that typing/spelling errors accounted for a third of lotal errors, and phonetic errors (consonant and vowel errors) accounted for another third. The remainder were semantic errors, added or deleted words, or unclassifiable. Further analysis of consonant errors did not reveal any particular type of consonant to be more susceptible to error than others. Male talkers had significantly worse keyword scores than females but did not have significantly greater total error counts indicating more errors on function words for females. The difference between high and low intelligibility speakers (by total error count) was accounted for by increased typing/ spelling and consonant errors. Acoustic analysis of incorrectly transcribed 
words revealed that phonetic errors appeared to originate in the mouths of speakers while errors like word substitutions arise in the ears (or brains) of listeners.

5aSC5. Intelligibility of normal speakers: Vowel space characteristics. Gina M. Torretta, Ann R. Bradlow, and David B. Pisoni (Speech Res. Lab., Psych. Dept., Indiana Univ., Bloomington, IN 47405)

Even within careful speaking conditions, normal speakers vary in intelligibility. This study investigated vowel space characteristics as one correlate of variation in intelligibility. Data consisted of 100 Harvard sentences, spoken by 20 speakers and transcribed by ten listeners per speaker. Formant measurements (mel transformed) of six tokens each for three vowels $[i, a, 0]$ provided a basis for assessing the vowel-space characteristics. First, vowel-space area was calculated as the Euclidean area between vowel category means. Across all 20 speakers, this area did not correlate with intelligibility. However, for a subset of the speakers (three highest, three lowest in intelligibility) there was a moderate positive correlation $(r=+0.34)$. Second, vowel-space dispersion was calculated as the mean distance from the center of the triangular space to all 18 tokens. This dispersion measure showed a moderate, positive correlation with intelligibility across all 20 speakers $(r=+0.43)$, suggesting the importance of individual token location for overall intelligibility. Finally, minimum and maximum formant values gave a measure of $F 1$ and $F 2$ range covered by vowel tokens. This measure revealed that $F 1$ range correlated positively with intelligibility $(r=+0.53)$, whereas $F 2$ range did not. The results will be discussed in the context of talker variability and intelligibility.

5aSC6. Visual-speech intelligibility for syllables: A comparison of conversational and clear speech. Jean-Pierre Gagné (École d'Orthophonie et d'Audiologie, Université de Montréal, Montréal, PQ H3C 3J7, Canada) and Anne-Josée Rochette (Université de Montréal, Montréal, PQ H3C 3J7, Canada)

The visual speech intelligibility of $/ \mathrm{C}-\mathrm{v} /$ and $/ \mathrm{C}-\mathrm{v}-\mathrm{C} /$ syllables spoken under conversational-like and clear speech conditions was compared. The syllables consisted of voiced consonants, varying in place of articulation (i.e., $/ b, d, g, v, z, j /)$, presented in three vowel contexts $(/ a, i, w /)$. Talkers were six female adults, and each produced four iterations of the stimulus set for each of the two speaking conditions. The 1728 videotaped items ( 36 syllables $\times 2$ manners of speech $\times 4$ iterations $\times 6$ talkers) were edited, randomized, and shown (without sound) to viewers with normal hearing. Viewers' completed a consonant-recognition task and these data were used to determine a visual speech-intelligibility score for each stimulus set produced under the conversational and clear speech conditions. Results revealed that, despite significant within-talker variability in both speaking conditions, significantly higher speech intelligibility scores were observed for the production of clear speech. A significant talker effect, and a talker $x$ manner interaction also emerged. The magnitude of the clear speech effects and the within- and across-talker variability in visual-speech intelligibility will be discussed.

5aSC7. Investigating the role of specific facial information in audio-visual speech perception. P. M. T. Smeele, Lisa D. Hahnlen, Erica B. Stevens, Patricia K. Kuhl (Dept. of Speech and Hearing Sci., Univ. of Washington, Seattle, WA 98195), and Andrew N. Meltzoff (Univ. of Washington, Seattle, WA 98195 )

When hearing and seeing a person speak, people receive both auditory and visual speech information. The contribution made by visual speech information has been demonstrated in a wide variety of conditions, most clearly when conflicting auditory and visual information is presented. In this study an investigation was performed to determine which aspects of the face most strongly influence audio-visual speech perception. The visual stimulus was manipulated using special effects techniques to isolate three specific "articulatory parts:" lips only, oral cavity only, or jaw only. These "parts" and their combinations were dubbed with auditory tokens to create "fusion" stimuli (A/aba/ + V/aga/) and "combination" stimuli (A/aga/ + V/aba/). Results indicated that visual information from jaw-only movements was not sufficient to induce illusory effects. However, for the combination condition, seeing moving lips or the inside of the speaker's mouth produced substantial audio-visual effects. Additional visual information from other articulators did not significantly increase the effect. In the fusion situation, both the lips and oral cavity were necessary to obtain illusory responses, whereas individually they produced very few. The results suggest that visual information from the lips and oral cavity together are sufficient to influence auditory speech processing. [Research supported by NICHD.]

5aSC8. A comparison between cerebral-palsied and normal adults in the perception of auditory-visual illusions. Nithya Siva, Erica B. Stevens, Patricia K. Kuhl (Dept. of Speech and Hearing Sci., Univ. of Washington, Seattle, WA 98195), and Andrew N. Meltzoff (Univ. of Washington, Seattle, WA 98195)

Listeners obtain information about speech both from listening to a talker and by using visual cues from the talker's face. As demonstrated in the McGurk effect, conflicting auditory and visual cues produce illusions. The present experiment investigated whether lack of experience with normal speech production affects the perception of auditory-visual illusions. Adults with cerebral palsy (CP) who have been severely dysarthric since birth were compared to normally speaking adults on two types of illusions: (1) auditory /aba/ paired with visual /aga/ which typically produces a /da/ illusion; and (2) auditory /aga/ paired with visual /aba/ which typically produces a / $\mathrm{bga} /$ illusion. The number of illusory responses was compared for each group. There was no difference between groups in the number of $/ \mathrm{da} /$ illusions. However, adults with $\mathrm{CP}$ perceived fewer $/ \mathrm{bga} /$ illusions than normal adults. These results suggest that lack of experience articulating speech inhibits a listener's ability to perceive unusual English phoneme clusters like /bga/. [Research supported by NICHD.]

5aSC9. Vowel perception in dyslexic subjects. Carol B. Bertucci (MGH Inst. of Health Professions, 101 Merrimac St., Boston, MA 02114)

It has been demonstrated by some investigators that poor readers may have less well-defined phonological categories than good readers. Whereas these studies have considered the perception of consonants, the current study investigated the percetion of the vowels $/ \mathrm{I}, \varepsilon, \mathfrak{e} /$ by individuals with dyslexia. Perception was also compared with production and with other measures considered important for reading. A small-group case-study design was employed, in which the patterns of performance of one adult and three children with varying degrees of dyslexia were examined individually and compared with age-matched control subjects. An identification task was used, in which subjects matched synthesized stimuli, equally spaced along a continuum of $F 1 / F 2$, to pictures of pit, pet, and pat. A set of intermediate stimuli was administered at a later date. The findings of this exploratory study suggest that subjects vary in their ability to perceive the vowels $/ \mathrm{r}, \varepsilon, \mathfrak{x} /$ according to the severity of their reading/spelling impairment. Differences in perception were noted in the sharpness of the boundaries between phonemes and in the tendency to give abberant responses to test stimuli. Shifts in perception were also noted across days and stimulus sets in some individuals.

5aSC10. Stress identification by hearing-impaired listeners. Dragana Barac-Cikoja (Ctr. for Auditory and Speech Sciences, Gallaudet Univ., 800 Florida Ave. NE, Washington, DC 20002) and Sally Revoile (Gallaudet Univ., Washington, DC 20002)

Some acoustic correlates of stress perception were studied for listeners with moderate to profound hearing loss. The sentence "You put VCV to bed" was spoken with emphasis on each of the main constituents (you, put, VCV, to bed) respectively, yielding four stress contexts. For each stress context, ten different sentence utterances were tested. The sentences were modified acoustically to neutralize the temporal and/or amplitude prominence of the stressed word. Hearing-impaired $(n=22)$ and normal-hearing listeners $(n=8)$ identified the stressed word for the modified and unmodified sentences. Contrary to the performance in normal-hearing listeners, stress perception by the hearing-impaired listeners was significantly reduced when both amplitude and temporal characteristics of the stressed word were modified. Less of an effect occurred when only amplitude or temporal characteristics alone were neutralized. Results are discussed with reference to the extent and rate of $F 0$ change across the sentence for the four stress contexts, and the accessibility of the intonational cues to the hearing impaired subjects as predicted by several audiological and psychoacoustic variables. [Work supported by NIH.] 
5aSC11. Effects of formant bandwidth on the performance of two cochlear implant processing strategies. John W. Hawks (Kent State Univ., School of Speech Pathology and Audiology, Kent, OH 44242), Marios S. Fourakis (Ohio State Univ., Columbus, OH 43210), Margaret W. Skinner, Timothy A. Holden, and L. K. Holden (Washington Univ. School of Medicine, St. Louis, MO 63110)

The aim of the experiment reported here was to examine the responses to synthetic vowels of the processor of the Nucleus Cochlear Implant System, using the MPEAK and SPEAK speech coding strategies. In previous work with natural vowels (Skinner $e t$ al., submitted to Ear Hear.), it was found that second formant information was better transmitted using the SPEAK strategy, while first formant information was better transmitted using the MPEAK strategy. Examination of the output of the SPEAK processor showed activation of multiple adjacent electrodes resulting in less distinct spectral cues than with MPEAK. This prompted the present investigation, which uses synthetic vowels with varying formant bandwidths. Four synthetic vowels, which had been identified consistently by normal hearing subjects as one of $[\mathrm{L}, \varepsilon, \mathrm{U}, \mathrm{\Lambda}]$ were used as anchor points for the creation of continua in which the bandwidths of the first and second formants were systematically and independently decreased in steps of $10 \%$ to a minimum of $50 \%$ of the formant bandwidths of the anchor stimuli. Results from the processors and identifications from subjects using the two processing stategies will be presented and compared. [Work supported by $\mathrm{NIH}$.

5aSC12. Creating and confirming acoustic targets in compression amplification. Jerry L. Yanz and David J. Delage (Qualitone, $4931 \mathrm{~W}$. 35th St., Minneapolis, MN 55416)

The recent increase in the use of compression amplifiers in hearing aids has created the need for more sophisticated fitting algorithms to help select the hearing aid characteristics-gain, output, compression threshold and compression ratio-most appropriate for individual hearing losses. Conventional prescriptive formulas (NAL-R, POGO, etc.), intended for linear amplifiers, are not adequate. The Independent Hearing Aid Fitting Forum (IHAFF) recently published a protocol for defining these variables using multiple existing clinical instruments, computer software, and normative corrections. In this presentation the Prophet Hearing Aid Fitting System will be described. Prophet was developed to accomplish the goals of the IHAFF protocol, as well as the FIG6 approach of Killion, in a single integrated, PC-based instrument. The system sharpens the accuracy of amplification targets by calibrating all signals in dB SPL with real ear values where appropriate, thereby eliminating any reliance on inherently variable $\mathrm{HL}$ norms. In addition to creating acoustic targets, it confirms the fit to target by measuring the response of the hearing aid to a multi-level, speech-noise stimulus.

5aSC13. Results of take-home trial for a nonlinear beamformer used as a noise reduction strategy for cochlear implants. Vena Margo, Mark Terry, Christopher Schweitzer (AudioLogic, 6655 Lookout Rd., Boulder, CO 80301), and Jon Shallop (Denver Ear Inst., Englewood, CO 80110)

A nonlinear frequency domain beamforming algorithm was evaluated as a noise reduction technique with eight cochlear implant patients, in a take-home trial. The subjects wore the prototype device coupled to the Nucleus processor for 5-8 weeks. The subjects were tested both before and after the take-home trial with a single noise source at 45 deg ipsilateral to the implant and with sentence type material. The results strongly favored the coupled device for noisy environments and indicated no additional benefits from a period of continued use. The subjective reports indicated that the two-microphone coupled device had a more robust sound quality and is preferred in noisy environments to the stand alone device. Currently the performance measures are being extended to cover sentence scores in a diffuse noise field (four speakers at different locations) and the results for both noise jammer arrangements will be reported.
5aSC14. Temporal characteristics of the speech of a deaf-blind person with a cochlear implant. Lynn Limbach, Chavi Goodman Soffer (Dept. Speech, Commun. Sci., \& Theatre, St. John's Univ., Jamaica, NY 11439), Nancy S. McGarr, and Fredericka Bell-Berti (St. John's Univ., Jamaica, NY 11439 and Haskins Labs., New Haven, CT 06511)

Many of the early studies of the effects of cochlear implants on speech production focused on the speech of persons with profound adventitious hearing loss. More recently, there has been substantial interest in the effects of implants on the speech of persons with congenital hearing loss. This study extends the research in this area to a woman who has been profoundly hearing impaired since birth and sustained a sudden loss of vision in her late teens. She has received extensive speech training, and is also a fluent reader of Braille. For this study, she was recorded reading a passage aloud one month postimplant, and then twice again at three month intervals. During each recording session, she read the six-sentence passage with the implant activated and then with the implant tumed off. Analyses of temporal characteristics of each sentence, including relative speech and pause durations and word durations, will be presented. [Work supported by St. John's University and by NIH Grant DC-00121 to the Haskins Laboratories.]

5aSC15. Esophageal speech analysis. Costas Pastiadis, Zafiris G. Politis, and George Papanikolaou (Dept. of Elec. and Comput. Eng., Aristotle Univ. of Thessaloniki, Univ. Campus, 54006, Thessaloniki, Greece)

Aspects of esophageal speech are investigated in this paper. Esophageal speech is produced by laryngectomized people who utter by expelling air constricted under the entrance of the esophagus, forcing the cricopharyngeal muscle to oscillate equivalently to vocal cords in normal speakers. Nine male esophageal speakers were used for the analysis. Spoken material consisted of Greek vowels and syllables ( $\mathrm{CV}, \mathrm{CCV}, \mathrm{VC})$, each repeated three times continuously by each speaker. $F_{0}$ values and plots were obtained for all speakers using central clipping autocorrelation, cepstrum analysis, and a modified Hilbert transform envelope method that seemed to give more consistent results among others. Most frequently observed $F_{0}$ values varied from speaker to speaker with an average of 73 Hz. $F_{1}$ vs $F_{2}$ plots for Greek phonemes $\alpha, \epsilon, \iota, 0,0 v$ were obtained by LPC. Deviations from normal speakers were very small. Significant similarity to English equivalent phonemes was also observed. Speech power versus time slope for vowel-type utterances was investigated as a measure of power reduction rate, showing an average of $-86 \mathrm{~dB} / \mathrm{s}$. Finally, implications about source volume velocity are made using LPC inverse filtering. Cepstrum analysis revealed a $-6-\mathrm{dB} /$ oct voice source spectral tilt instead of $-12 \mathrm{~dB} /$ oct for normal speakers.

5aSC16. Acoustic properties of alaryngeal speech. Nancye C. Roussel (Dept. of Commun. Disord., Univ. of Southwestern Louisiana, Lafayette, LA 70504)

One of the current options for the restoration of speech in individuals. who have undergone total laryngectomy, is the use of artificial (electronic) larynx devices. These devices can be classified as either transcervical devices, which transmit the acoustic signal to the vocal tract through the skin and other tissues of the neck, or intraoral in which the signal is directed into the oral cavity through a short piece of plastic tubing. Differential effects on single word intelligibility as a function of artificial larynx type were studied. All judges had no previous experience listening to alaryngeal speech. Four brands of the artificial larynx devices were tested and the results revealed a significant increase in intelligibility with the use of transcervical devices. These results were somewhat surprising as earlier informal surveys had intraoral devices rated higher in terms of listener preference. Perceptual error matrices were constructed and are being analyzed, as are acoustic representations of test stimuli to determine possible factors to account for these intelligibility differences. Results of these analyses will be presented. 
5aSC17. Analytical study of pathologic tremulous voices of Japanese-speaking patients. Hiroko Kosaki, Kimitaka Kaga (Dept. Otolaryngol., Univ. of Tokyo, Tokyo 113, Japan), Seiji Niimi, Masanobu Kumada (Univ. of Tokyo, Tokyo 113, Japan), and Hajime Hirose (Kitazato Univ., Sagamihara 228, Japan)

This study was undertaken to clarify the major characteristics of voices of the Japanese-speaking patients of the adductor spasmodic dysphonia. The mechanism of the tremulous voice was also studied. For Japanesespeaking patients, to make a proper diagnosis of adductor spasmodic dysphonia, an evaluation method of the grade of the disease is needed. Sound spectrographic analysis was applied. First, the voices of the adductor spasmodic dysphonia patients were compared with the voices of other tremulous voice disease patients. Second, the voices of the different stages of adductor spasmodic dysphonia were analyzed. In the first study, results revealed that adductor spasmodic dysphonia voices showed irregularity of the fluctuation frequency in both pitch and intensity, while the voices with essential voice tremor showed a periodic fluctuation in pitch and intensity. In the second study, it was indicated that the cessation and fine flutter of the voice have a major role in the grade of the disease. With those results, phonological measurement and clinical feature can be matched. By evaluation of the irregularity of the voice, the diagnosis of tremulous voices might be confirmed. It was suggested that contributing factors to the voice symptom were not uniform.

5aSC18. The structural organization of the mental lexicon and its contribution to age-related declines in spoken word recognition. Mitchell S. Sommers (Dept. of Psych., Washington Univ., St. Louis, MO 63130)

Three experiments were conducted within the framework of the Neighborhood Activation Model of spoken word recognition to examine how the structural organization of the mental lexicon may contribute to age-related declines in spoken language processing. According to the model, lexically hard words are items that are phonetically similar to many other highfrequency words and should be identified less accurately than easy words which are phonetically similar to relatively few, low-frequency items. The results of experiment 1 demonstrated that lexical difficulty had differential effects on older and younger listeners. Specifically, relative to young listeners, older adults exhibited significantly poorer identification scores for lexically hard items. Experiment 2 indicated that these age differences in the effects of lexical difficulty were maintained under conditions in which performance for easy words was the same for older and younger listeners. In the third experiment, reducing the resources available for perceptual identification, by changing from single to multiple talkers, produced greater effects of lexical difficulty for older, compared to younger, listeners. Explanations for the results based on diminished cognitive resources and impaired inhibitory control will be discussed. [Work supported by Washington Univ.]

5aSC19. Age differences in the effects of talker familiarity on spoken word recognition. Cynthia A. Yonan and Mitchell S. Sommers (Dept. of Psych., Washington Univ., St. Louis, MO 63130)

Recent evidence has demonstrated that young adults achieve improved spoken word recognition performance when words are produced by familiar, as compared to unfamiliar, voices [Nygaard et al., Psych. Sci. 5, 42-45 (1994)]. This benefit for familiar voices is hypothesized to be a result of reduced perceptual normalization demands when listeners have previous knowledge of source characteristics. The present study investigated whether normal-hearing older listeners exhibit a similar improvement for familiar voices. Younger and older volunteers were trained to discriminate four voices and were then tested on a perceptual identification task with familiar and unfamiliar voices. Although voice discrimination did not differ as a function of age, only the older participants exhibited improved word recognition performance for familiar talkers. Word recognition performance was also examined separately for words at two levels of lexical difficulty: (1) words that are phonetically distinct from others (easy words), and (2) words that are phonetically similar to others (hard words). The largest benefit of voice familiarity was observed for older listeners identifying lexically hard words. The implications of these findings for age differences in the use of voice information will be discussed.
5aSC20. Closed-set response formats: An evaluation within the framework of the neighborhood activation model. Rebecca $S$. Prohofsky and Mitchell S. Sommers (Dept. of Psych., Washington Univ., St. Louis, MO 63130)

It has been suggested that open- and closed-set response formats engage distinct perceptual mechanisms during spoken word recognition. One finding consistent with this hypothesis is that lexical difficulty (neighborhood density and neighborhood frequency) reduce identification performance in open- but not closed-set formats [Kirk et al., Ann. Otol. Rhinol. Laryngol. (in press)]. An altemative explanation for the differential effects of response format on lexical difficulty in this earlier study, however, is that the response alternatives were not systematically selected to maximize confusability with the target items. Therefore, the present investigation compared the effects of lexical difficulty in open- and closed-set response formats when the response alternatives in the closed-set condition were the five words most confusable with the target item. Preliminary results indicate that, relative to the open-set condition, when the difficulty of alternatives is controlled, identification within the closed-set format is improved for hard, but not for easy, words. It is hypothesized that the improved word recognition for hard words in closed-set formats is the result of reducing the viable candidate set. In contrast, the already small candidate set of easy words minimizes the benefits of reducing the number of viable candidate items.

5aSC21. Implicit memory for hybrid silent-center syllables. Susan L. Hura (Dept. Audiology and Speech Sciences, Purdue Univ., 1353 Heavilon Hall, West Lafayette, IN 47907-1353)

A growing body of evidence suggests that information about specific voices and specific exemplars of words is encoded in memory, as observed in results of implicit memory tasks in which subjects perform perceptual classification of stimuli. Hura [dissertation, University of Texas (1994)] found identification performance on silent-center (SC) syllables improved with previous exposure to corresponding full syllables, implying that representations of individual full syllables were encoded in memory and accessed in identification of SC stimuli. The current study investigates whether prior exposure to $\mathrm{SC}$ syllables improves performance on full syllables. Ten $/ \mathrm{bVt} /$ syllables spoken by multiple talkers were used to generate SC and hybrid SC stimuli (which combine the initial CV transition from one talker and the final VC transition from another). A subset of SC syllables, varying for identity of talker or vowels presented, were presented to subjects for identification. After a variable delay subjects were tested on a subset of full syllable stimuli. The magnitude of implicit memory effects in each case is assessed to address the claim that dynamic vowel information is speaker independent as suggested by the theory of dynamic specification.

5aSC22. Effects of compound priming on listeners' identification of a $/ \mathrm{kVC} /$ continuum. Terrance M. Nearey, Michael Kiefte, Christian Guilbault, Kaori Kabata, and Zhang Xia (Dept. of Linguist., Univ. of Alberta, Edmonton, AB T6G 2E7, Canada)

A continuum was synthesized to span the words cop, cup, cob, and $c u b$. Two cues were varied in six steps each. Vowel F2 ranged from 960 to 1160 Hz. Voice bar duration for the final consonant ranged from 10 to $60 \mathrm{~ms}$. Other properties approximated the average the four words pronounced by a male speaker in the compound nouns: traffic cop, tea cup, corn cob, and bear cub. The four context words, traffic, tea, corn, and bear, were also synthesized. Thirteen listeners categorized ten replications of the stimuli in each of five contexts: as isolated words and following each of the context words. As expected, words are favored in appropriate contexts. Logistic regression indicates that about $96 \%$ of variance in listeners' responses can be attributed to phoneme-level stimulus effects and to stimulusindependent (phonological and lexical) biases. There is also evidence for small changes in sensitivity to cues as a function of lexical context. The latter result is of interest for certain alternative models [D. Massaro, Cog. Psych. 23, 558-564 (1991)]. However, observed changes in sensitivity do not relate well to predictions from any existing theory. [Work supported by SSHRC.] 
5aSC23. Task demands affect lipreaders' search for information. Charissa R. Lansing, Heather D. Minch (Dept. of Speech and Hearing Sci., Univ. of Illinois at Urbana-Champaign. Champaign. IL 61820), and George W. McConkie (Univ. of Illinois at Urbana-Champaign, Urbana, IL 61801)

Two experiments were conducted to determine whether a lipreader's task (making phonemic or prosodic distinctions about the spoken message) changes the regions of the talker's face from which information is needed, and whether lipreaders are sensitive to these changes. An initial experiment found that when face movement was limited to the talker's lips-plusmandible region, the accuracy of intonation judgments was reduced but the accuracy of phonemic judgments was not. A second experiment then recorded gaze direction of lipreaders as they attempted to make judgments about the phonemic, stress, or intonation contour characteristics of short utterances. Results indicated that they spent more time looking at upper portions of the face when judging intonation contours than in the other conditions. Other differences in the eye movement patterns were also found among task conditions. Thus lipreaders are aware that different aspects of the speech information are signaled at different facial regions, and can adjust their direction of gaze to take advantage of this knowledge. [Work supported by NIH.]

5aSC24. Detection of speech targets under the divided attention manipulations. Chin-Hsing Tseng and Feng-Ming Tsao (Commun. Disord., Dept. of Special Education, National Kaohsiung Normal Univ., Kaohsiung, Taiwan)

Divided attention tasks were designed to test the following hypothesis: When two dimensions of a single speech stimulus are simultaneously attended to for target detection, one dimension can be processed faster than the other if the former has a higher information value. Targets were (1) a given vowel or any vowels produced by a given speaker, or (2) a given plosive or a specified lexical tone embedded in a syllable, or (3) a specified lexical tone embedded in disyllabic noun or a name for man-made objects. The target occurrence probabilities for the two dimensions were set at 0.10 vs $0.40,0.25$ vs 0.25 , or 0.40 vs 0.10 in a given block of trials. Chinese- speaking college students participated in these experiments. It was found that the target detection latency varied inversely with target occurrence probability for either dimension under all these manipulations. A clear performance trade-off pattern was observed for each manipulation: that is. as one dimension received more attention, the processing of the other became less efficient. These results demonstrated that attention can be divided between dimensions of "speech processing space" as cued by the information value. [Work supported by NSC, ROC.]

5aSC25. Recent progress in the INRS speech recognition system. Douglas O'Shaughnessy, Zhishon Li, and Azarshid Farhat (INRS-Telecommunications, 16 Pl. du Commerce, Verdun, PQ H3E 1H6, Canada)

For large-vocabulary continuous-speech recognition, a two-pass search allows inexpensive first-pass models, with pruned search spaces represented by word graphs. Powerful language models and detailed acousticphonetic models follow. A first-pass Viterbi lexicon search is avoided via tables of estimates of phone scores and durations, from backward-Viterbi searches of much smaller graphs, which impose diphone rather than full lexical constraints on phonetic transcriptions. These estimates of phone scores and durations are used to calculate approximate acoustic malches for arbitrary phonetic transcriptions (one floating-point operation/phone). The speaker-independent system uses WSJ0 data (5000-word vocabulary), with separate male and female models: 3-state full right-context models, and code books of 14 static and 15 dynamic cepstral parameters. The first pass uses VQ models with one covariance matrix and 256 means. The word inclusion rate is about $97 \%$. For the second pass, trigram language models with perplexity 104 and continuous-HMM acoustic models achieved about $90 \%$ word-recognition accuracy on the development set. To achieve good trade-offs between acoustic models' complexity and trainability, a shared-distribution approach for clustering has distortion measures based only on the weights of Gaussian mixtures rather than all parameters. Word accuracy increased by $6 \%$ for the ATIS corpus. [Work supported by NSERC-Canada.]

FRIDAY MORNING, 1 DECEMBER 1995

ST. LOUIS E, 8:00 A.M. TO 12:00 NOON

\title{
Session 5aUW
}

\section{Underwater Acoustics: Seafloor Scattering}

\author{
Shira L. Broschat, Chair \\ School of Electrical Engineering and Computer Sciences, Washington State University, Pullman, Washington $99164-2752$
}

Contributed Papers

8:00

5aUW1. Seafloor backscatter measurements using explosive line arrays. Charles W. Holland and Peter Neumann (Planning Systems, Inc., 7923 Jones Branch Dr., McLean, VA 22102)

Current survey techniques typically measure seafloor backscattering from 30-50 deg in grazing angle. Low-angle measurements (less than about $30 \mathrm{deg}$ ) of seafloor backscattering require vertical directionality in the source and/or receiver to eliminate paths that have more than one bottom interaction. Several experiments using explosive line arrays (ELA) have been conducted to explore the potential for measurement of lowangle seafloor scattering. Air deployable explosive line arrays are attractive because they are relatively inexpensive, are command activated, and appear to be quite stable from ping to ping. Seafloor scattering measurements conducted with the ELAs will be compared with co-located measurements using SUS as well as a low-frequency coherent source array. [Work supported by the ONR/AEAS Program.]
8:15

5aUW2. Scattering strength cannot depend on the length of a pulse. Frank S. Henyey, Eric I. Thorsos, and K. Miguel Nathwani (Appl. Phys. Lab., Univ. of Washington, 1013 NE 40th St., Seattle, WA 98105)

Reilly ef al. [J. Acoust. Soc. Am. 97, 3385-3386 (A) (1995)] presented experimental results for low-frequency bottom scattering strength which appear to show pulse length dependence. It is shown that the scattering strength is independent of pulse length under the assumptions of (1) linear acoustics, and either ( $2 \mathrm{a}$ ) the scatterers are not moving, as for ocean bottom scattering, or (2b) the statistics of the scatterers are time independent (a stationary process) and uncorrelated to pulse timing, as for ocean surface zone scartering. These results hold if the pulses have the same frequency spectrum. In the usual case that their spectra are slightly different, rapid frequency dependence can appear to give pulse length dependence. In case (2a), the ensemble average removes that part of rapid frequency dependence due to random interference between returns from different scatterers or different parts of the same scatterer that appear in a single 
return. Rapid variation of the average received power can bias the measured scattering strength for longer pulses, but this effect can be removed from the data. Statistics other than scattering strength, such as peak return, are generally pulse length dependent

\section{8:30}

5aUW3. High-frequency bistatic scattering by sub-bottom gas bubbles. Dezhang Chu (Dept. of Appl. Ocean Phys. and Eng., Woods Hole Oceanograph. Inst., Woods Hole, MA 02543), Kevin L. Williams (Univ. of Washington, Seattle, WA 98105), D. Tang (Woods Hole Oceanograph. Inst., Woods Hole, MA 02543), and Darrell R. Jackson (Univ. of Washington, Seattle, WA 98105)

The acoustic scattering by sub-bottom inhomogeneities has received increased attentions in recent years. However, most of the previous studies were restricted to monostatic or backscattering. To better understand the complicated scattering process, a bistatic scattering involving both grazing and azimuth angles dependence is desirable. As part of the Coastal Benthic Boundary Layer Special Research Program (CBBLSRP), along with a previously reported backscattering experiment, a bistatic scattering field experiment using Mobile Benthic Receiver Arrays (MBRA) operated at 40 $\mathrm{kHz}$ was conducted at Eckernfoerde Bay, Germany in April 1993. It has been found in previous studies that high-frequency backscattering measured is due to methane gas bubbles buried about a meter beneath the seafloor. In this paper, a bistatic scattering model is proposed as an extension of the previously developed backscattering model to account for the three-dimensional (out-of-plane) scattering effect. In the model, as in the backscattering model, gas bubbles were assumed to be oblate spheroids with varying aspect ratios. While the parameters used in the model to fit backscattering data are kept unchanged, the model prediction for the bistatic scattering is satisfactory. It is found that the scattering strength exhibits a mild azimuthal dependence and is sensitive to the scattering geometry. [Work sponsored by ONR.]

\section{$8: 45$}

5aUW4. Measurements of the acoustic $(200 \mathrm{kHz})$ backscatter from a carbonate sediment at low grazing angles. Robert $\mathrm{A}$. Altenburg and Nicholas P. Chotiros (Appl. Res. Labs., Univ. of Texas, P.O. Box 8029, Austin, TX 78713-8029)

A multibeam sonar, mounted on a remotely operated vehicle, and operated at $200 \mathrm{kHz}$ was used to acquire bottom backscatter data at nine different sites in the Dry Tartugas near Key West, Florida. The bottom in this area is carbonate mud and biogenic carbonate detritus (hermitific corals, shells, etc.). Bottom backscattering strength as a function of grazing angle for each site, supported by bottom core sample analyses, is presented. A wide range of bottom backscaltering strength values were measured and some correlation between bottom type and backscattering strength was found. [Work sponsored by ONR under management by NRL/SSC under the Coastal Benthic Boundary Layer Special Research Program.]

\section{9:00}

5aUW5. Acoustical ray-tracing insonification software modeling of reverberation at selected sites near the Mid-Atlantic Ridge. Vincent H. Lupien, Joseph E. Bondaryk, and Arthur B. Baggeroer (Dept. of Ocean Eng., Rm. 5-435, MIT, 77 Massachusetts Ave., Cambridge, MA 02139)

The software package ARTIST is used to model reverberation in a series of pings at selected sites in both monostatic and bistatic configurations. ARTIST is a ray-tracing-based program incorporating array positions and orientations, sound-speed profiles, bathymetry, and beam patterns. Simulated beam-time data are generated by integrating the energy at the seafloor from both arrays, incorporating all sidelobe effects. It is shown that prominent features in the beamformed data match excellently with direct and surface-reflected paths to the seafloor and that these features are dominated by shadowing effects and average grazing angles. The received data show appreciable returns in regions where no direct or surfacereflected paths exist. It is postulated that these returns are part of an underlying, slowly varying reverberation envelope which changes little from ping to ping and is present in bistatic cases as well. Possible physical mechanisms leading to this behavior are presented. The scattering strength of selected seafloor features is presented along with its dependence on grazing and bistatic angles.

\section{$9: 15$}

5aUW6. An integrated modal approach to waveguide reverberation. Brian H. Tracey and Henrik Schrnidt (Dept. of Ocean Eng., MIT, 77 Massachusetts Ave., Bldg. 5-007, Cambridge, MA 02139)

In shallow-water environments scattering from rough surfaces and volume inhomogeneities acts to degrade sonar performance. An efficient normal mode method has been developed to model the resulting reverberation. The self-consistent perturbation theory of Schmidt and Kuperman [J. Acoust. Soc. Am. 97, 2199-2210 (1995)] has been reformulated in terms of normal modes to handle scattering from rough fluid and elastic interfaces. A similar, recently developed volume scattering theory is used to model the effects of sound speed and density fluctuations in the seabed and water column. The two theories are integrated numerically and used to contrast the different scattering mechanisms. Waveguide propagation effects are seen to be extremely important. Spatial correlation and modal cross-correlation statistics are estimated and used to study decorrelation of the sound field. Excellent agreement with the wave-number integration implementation by Schmidt and Kuperman is demonstrated for the case of rough interface scattering, with the modal approach requiring significantly less computation for low-frequency shallow-water scenarios.

\section{9:30}

5aUW7. Low-frequency, direct-path acoustic reverberation near the Mid-Atlantic Ridge. Jerald W. Caruthers (Naval Res. Lab., Stennis Space Center, MS 39529) and Jorge C. Novarini (PSI, Long Beach, MS 39560)

In 1991 and again in 1993, the Acoustic Reverberation Special Research Program (ARSRP) of the Office of Naval Research conducted lowfrequency acoustic reverberation experiments just west of the Mid-Atlantic Ridge. To support analyses of these measurements, the ARSRP also conducted geological and geophysical surveys of the region, resulting in nearly full-coverage bathymetry gridded to a resolution of $200 \times 200 \mathrm{~m}$. Direct-path reverberation data have been modeled successfully using Lambert's law applied to the local grazing angle determined from the highresolution bathymetry. Excellent agreement of this simple model with data shows clearly that, for this region, knowledge of the 2-D seafloor morphology at the proper scale determines the ability to predict reverberation. For a model such as Lambert's law, which glosses over the details of seafloor microroughness or texlure at the scale of the acoustic wavelength $(\lambda=6 \mathrm{~m})$, the bathymetry scale that is critical is shown to be an order of magnitude or two above the acoustic scale. Lambert coefficients for the region varied depending on sedimentation and local geomorphology. For this region, explaining the success of Lambert's law and its regional differences might serve as a goal of more detailed theoretical efforts into scattering at the microscale. [Work supported by ONR.] 
5aUW8. Shallow-water reverberation due to sediment volume inhomogeneities. Dajun Tang (Dept. of Appl. Ocean Phys. and Eng., Woods Hole Oceanograph. Inst., Woods Hole, MA 02543)

This is a model of shallow-water reverberation due to sediment volume inhomogeneities. The scatterers are three-dimensional sound speed and density variabilities in the sediment. A first-order perturbation approximation is adopted assuming the sediment variabilities are small quantities. Within the applicable range of that approximation, this formalism is exact. It is found that the strength of the scattered field or reverberation strength can be expressed as the coupling between the incident and scattered normal modes. The mode coupling coefficient is found to be the same as the scattering coefficient sampled at relevant modal angles. In the development of the formulation, no empirical scattering function is involved. Also found is a dispersion factor associated with each mode in the mean reverberation curve corresponding to the spreading of the source pulse for each mode. Numerical examples are given for typical shallow-water environments.

\section{0:00-10:15 Break}

\section{0:15}

5aUW9. Sound scattering at a rough water-sediment interface in shallow water. X. Tang and F. D. Tappert (Appl. Marine Phys., Univ. Miami, RSMAS, 4600 Rickenbacker Cswy., Miami, FL 33149)

The method of smoothing is used to study sound propagation and scattering in a realistic shallow-water environment with a randomly rough water-sediment interface. A mean field wave equation for the coherent acoustic field is obtained that has a complex-valued attenuation coefficient that is localized at the mean interface. The solution of the mean field equation, under highly idealized conditions, is derived and discussed. The branch point and pole singularities are found to be related to the head wave mode and the Biot-Tolstoy rough boundary wave mode, respectively. Also obtained is the coherent plane-wave reflection coefficient which is found to vanish when the acoustic frequency and the incident angle of the plane wave satisfy a certain resonant condition. For testing the mean field equation, a stochastic full-wave forward propagation PE model is developed for Monte Carlo simulations. The numerical results show that the coherent field obtained by an ensemble average over many realizations is in agreement with that obtained by solving the mean field equation just once. The Monte Carlo simulations also yield additional useful information about higher-order moments of the acoustic field. [Work supported by ONR.]

\section{0:30}

5aUW10. Benchmarks for backscatter in parabolic evolution. Eric Smith (Appl. Res. Labs., Univ. of Texas, Austin, TX 78713-8029)

Recently, a method was described for improving parabolic equations to account for intermediate backscattering induced by slow range dependence of the acoustic index of refraction. The method has found difficulty gaining acceptance, though, because there have been no readily available benchmarks for the systems to which it applies. This paper presents a method for constructing a class of benchmarks, by means of which a parabolic equation can be used to test itself for correct evolution through variations in sound speed. The method makes use of the conformal equivalence of changes in acoustic index of refraction to changes in geometry. As examples within this class, a set of special cases is treated analytically. These are exactly solvable models, in which intermediate backscattering leads to recognizable and physically significant effects. In particular, they can be used to show why energy conservation alone is not a sufficient requirement to produce correct parabolic evolution, even when it is physically appropriate. [Work supported by the ARL:UT Independent Research and Development Initiative.]
5aUW11. Modeling of three-dimensional seismoacoustic reverberation from anisotropic roughness patches. Henrik Schmidt and Huaiyu Fan (Dept. of Ocean Eng., MIT, Cambridge, MA 02139)

A perturbation approach to narrow- and wideband modeling of scattering from roughness patches on an interface separating a fluid and an elastic half-space has previously been developed [LePage and Schmidt, $\mathbf{J}$. Acoust. Soc. Am. 89, 1941(A) (1991)]. However, based on a 2-D Fourier transform formulation it allows for modeling of the scattered near-field only, and it prohibits incorporation of waveguide effects. Here, a coordinate transformation is presented, yielding a representation of the virtual seismic moment sources for the scattered field in cylindrical coordinates. This source representation is compatible with the 3-D version of the OASES/SAFARI code, which has consequently been modified to provide extremely efficient numerical simulation of seismoacoustic reverberation in shallow- and deep-water waveguides. This new model has been applied to model the reverberation from horizontal roughness patches in the ARSRP mid-Atlantic environment. The model's efficiency has allowed for Monte Carlo estimation of the statistical properties of the reverberation from patches with anisotropic roughness statistics, including mean reverberation intensity and spatial correlation. The effects of roughness anisotropy, bottom elasticity and the waveguide physics are discussed. The principal result of this theoretical analysis is the conclusion that Lambert's law is totally inadequate for representing bistatic bottom reverberation. This conclusion is consistent with the results of the analysis of the bistatic data from the ARSRP experiment, as discussed in the companion paper by Bondaryk et al. [Work supported by ONR.]

\section{1:00}

5aUW12. Estimation of bottom scattering characteristics from acoustic reverberation special research program bistatic data. Joseph E. Bondaryk, Ira Dyer, and Eugene Dorfman (Dept. of Ocean Eng., Rm. 5-435, MIT, 77 Massachusetts Ave., Cambridge, MA 02139)

For many years, sonar practitioners have used Lambert's law on a flat bottom to describe clutter due to bottom reverberation. This examination of a subset of the bistatic data from the $B^{\prime}$ site of the ARSRP natural laboratory examines the validity of this approximation. Eight pings transmitted by the CORY CHOUEST and received by the Alliance were chosen to cover the bistatic angular range of -90 to $+90 \mathrm{deg}$ with respect to $B^{\prime}$. Transmission loss and area corrections to a flat bottom of representative depth were calculated using the ARTIST ray-tracing program. These corrections were applied to the data to compute scattering strength in each beam-time bin. This scattering strength was averaged over areas with similar grazing angle to transmitter, grazing angle to receiver and bistatic angle. Again, these are angles to a flat bottom scenario. The scattering strength plotted versus these three angles shows that neither Lambert's law nor the generalized version by Nayar et al. describe bistatic scattering. [Research supported by ONR.]

\section{1:15}

5aUW13. A unified perturbation approach to volume and roughness scattering. Anatoliy N. Ivakin (Andreev Acoust. Inst., Shvernika 4, Moscow 117036, Russia)

A unified approach is developed to study scattering from a fluid medium with irregularities of two different types: volume inhomogeneities (spatial fluctuations of the compressibility and density) and roughness of the interfaces. The approach treats roughness as a perturbation of the volume acoustic properties in the vicinity of the flat (unperturbed) surface. This permits a description of the scattering problem in terms of a unique integral equation for both types of irregularities. In the case of small roughness, the first iteration of this equation gives results corresponding to the extended Born approximation but provides a new approach for the case of interfaces with large, smooth roughness. As an application, acoustic scattering from a seabed consisting of an arbitrary number of irregular layers with volume inhomogeneities and rough interfaces is considered. [Work partially supported by ONR.] 
5aUW14. Effects of shear elasticity on high-frequency bottom scattering. Anatoliy N. Ivakin (Andreev Acoust. Inst., Shvernika 4, Moscow 117036, Russia) and Darrell R. Jackson (College of Ocean and Fishery Sciences, Univ. of Washington, Seattle, WA 98105)

It is known that marine sediments can support both compressional and shear waves. However, published work on scattering from irregular elastic media has not examined the influence of shear on seabed scattering in detail. Here, a perturbation model for high-frequency sound scattering from an irregular elastic bottom is considered. The seabed is assumed homogeneous on the average and two kinds of irregularities are assumed to cause scattering: roughness of the water-seabed interface and volume inhomogeneities of the sediment mass density and the velocities of compressional and shear waves. The first-order small perturbation approximation is used to obtain expressions for the scattering amplitude and average intensity of the scattered field. The angular dependence of the backscattering strength is calculated for different types of sediments and the influence of shear elasticity is examined by comparison with the case of a fluid bottom. Shear effects on both roughness and volume scattering are found to be rather strong (up to 5-7 dB) for dense sandy sediments at near-critical and subcritical grazing angles. The difference between the angular dependencies of roughness and volume scattering is examined and features of interest for remote acoustic characterization of seabeds are noted. [Work supported by ONR.]
5aUW15. Target strength of fluid-filled spherical shells related to material parameters and alternate filling fluids. Gregory Kaduchak and Charles M. Loeffler (Appl. Res. Labs., Univ. of Texas, P.O. Box 8029, Austin, TX 78713-8029)

Fluid-filled, thin spherical shells have been studied for use as passive sonar targets with applications in underwater navigation and calibration [see, e.g., D. L. Folds et al., J. Acoust. Soc. Am. 73, 1147-1151 (1983)]. The present research investigates the target strength of fuid-filled spheres as a function of shell and fluid parameters to aid in choosing alternative filling fluids. (Partial motivation is from the recent discontinuation of several chlorofluorocarbons which are commonly used.) Calculations based on 3-D elasticity theory describe the target strength dependence on the internal fluid density and sound speed. Here both time and frequency calculations of the form function and time domain scattering signatures describe favorable fluid characteristics. These features relate to the thickness and density of the confining spherical shell. It is shown that internalfluid density requirements vary considerably for thin aluminum and stainless-steel shells. Several different filling fluids are tested in spherical shells with consideration given to effective target strength, safety considerations, and cost. 